\title{
Modeling Earthquake Ground Motion with an Earthquake Simulation Program (EMPSYN) that Utilizes Empirical Green's Functions
}

\author{
Lawrence Hutchings
}

1992

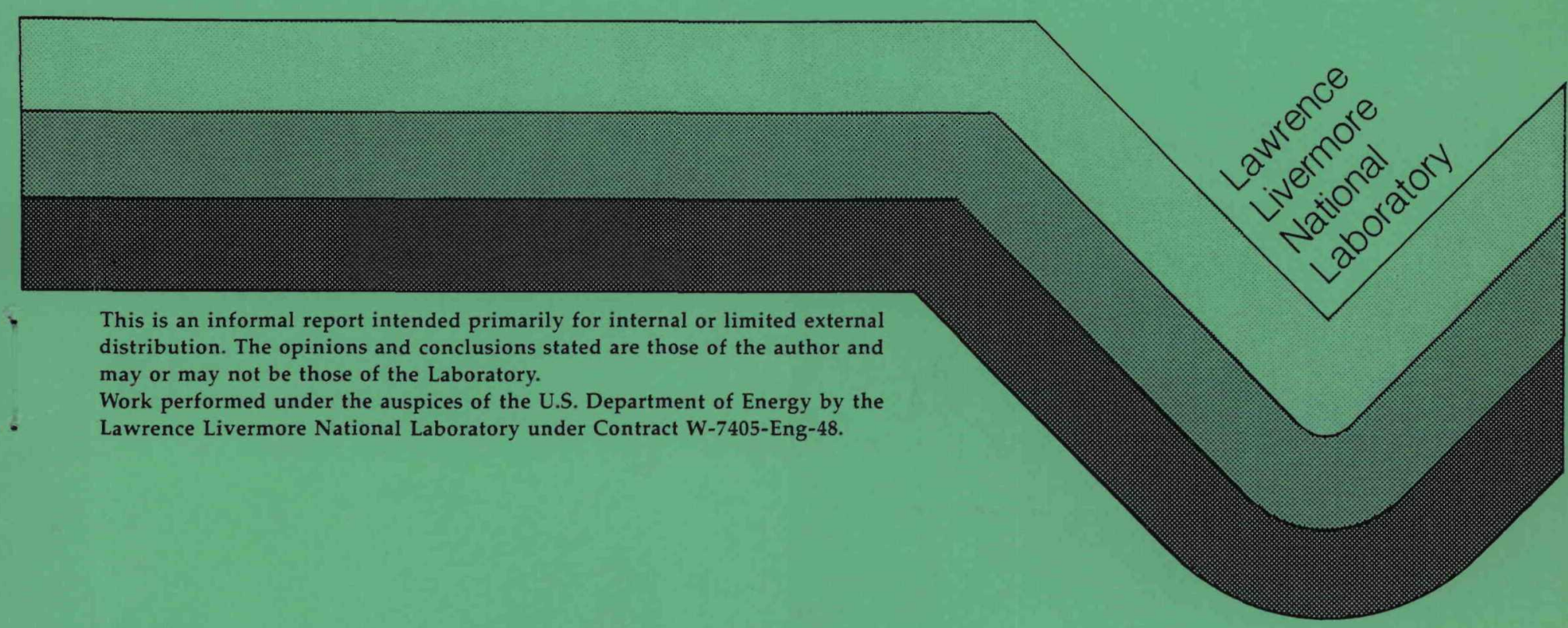




\section{DISCLAIMER}

This document was prepared as an account of work sponsored by an agency of the United States Government. Neither the United States Government nor the University of California nor any of their employees, makes any warranty, express or implied, or assumes any legal liability or responsibility for the accuracy, completeness, or usefulness of any information, apparatus, product, or process disclosed, or represents that its use would not infringe privately own rights. Reference herein to any specific commercial products, process, or service by trade name, trademark, manufacturer, or otherwise, does not necessarily constitute or imply its endorsement, recommendation, or favoring by the United States Government or the University of California. The views and opinions of authors expressed herein do not necessarily state or reflect those of the United States Government or the University of California, and shall not be used for advertising or product endorsement purposes.

This report has been reproduced directly from the best available copy.

Available to DOE and DOE contractors from the Office of Scientific and Technical Information

P.O. Box 62, Oak Ridge, TN 37831

Prices available from (615) 576-8401, FTS 626-8401

Available to the public from the

National Technical Information Service

U.S. Department of Commerce

5285 Port Royal Rd.

Springfield, VA 22161 


\section{DISCLAIMER}

Portions of this document may be illegible in electronic image products. Images are produced from the best available original document. 


\title{
Modeling Earthquake Ground Motion with an Earthquake Simulation Program (EMPSYN) that Utilizes \\ Empirical Green's Functions
}

Lawrence Hutchings

UCRL-ID--105890

Lawrence Livermore National Laboratory

Earth Sciences Department

DE92 010358

P.O. Box 808

Livermore, California 94550

\begin{abstract}
This report outlines a method of using empirical Green's functions in an earthquake simulation program EMPSYN that provides realistic seismograms from potential earthquakes. The theory for using empirical Green's functions is developed, implementation of the theory in EMPSYN is outlined, and an example is presented where EMPSYN is used to synthesize observed records form the 1971 San Fernando earthquake.

To provide useful synthetic ground motion data from potential earthquakes, synthetic seismograms should model frequencies from 0.5 to $15.0 \mathrm{~Hz}$, the full wave-train energy distribution, and absolute amplitudes. However, high-frequency arrivals are stochastically dependent upon the inhomogeneous geologic structure and irregular fault rupture. The fault rupture can be modeled, but the stochastic nature of faulting is largely an unknown factor in the earthquake process. The effect of inhomogeneous geology can readily be incorporated into synthetic seismograms by using small earthquakes to obtain empirical Green's functions.

Small earthquakes with source corner frequencies higher than the site recording limit $f_{\max }$, or much higher than the frequency of interest, effectively have impulsive point-fault dislocation sources, and their recordings are used as empirical Green's functions. Since empirical Green's functions are actual recordings at a site, they include the effects on seismic waves from all geologic inhomogeneities and include all recordable frequencies, absolute amplitudes, and all phases. They scale only in amplitude with differences in seismic moment. They can provide nearly the exact integrand to the representation relation. Furthermore, since their source events have spatial extent, they can be summed to simulate fault rupture without loss of information, thereby potentially computing the exact representation relation for an extended source earthquake.
\end{abstract}

University of California, Lawrence Livermore National Laboratory, UCRL-ID-105890, 1988.

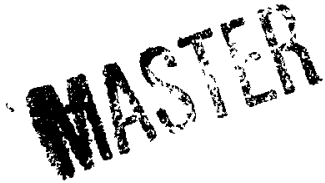




\section{Introduction}

To provide useful synthetic ground motion data from potential earthquakes synthetic seismograms should model frequencies from 0.5 to $15.0 \mathrm{~Hz}$, the full wave-train energy distribution, and absolute amplitudes. However, high-frequency arrivals are stochastically dependent upon the inhomogeneous geologic structure and irregular fault rupture. The fault rupture can be modeled, but the stochastic nature of faulting is largely an unknown factor in the earthquake process. The effect of inhomogeneous geology can readily be incorporated into synthetic seismograms by using small earthquakes to obtain empirical Green's functions. This report outlines a method of using empirical Green's functions in an earthquake simulation program EMPSYN, which provides realistic seismograms from potential earthquakes. Appendix A demonstrates the use of EMPSYN by synthesizing observed seismograms form the 1971 San Fernando earthquake. Appendix B lists the computer code.

Hutchings (1987) showed that aftershocks of the San Fernando earthquake recorded in the near-source region with moment below about $1.5 \cdot 10^{21}$ dyne-cm $\left(M_{L} \cong 2.5\right)$ effectively have impulsive point fault dislocation sources. Seismograms of these aftershocks include the full frequency range of effects from geologic structures and inhomogeneities, source-station geometry, and site-response that can be obtained at a recording site from an elemental source. It is not possible to decompose recordings of small earthquakes into their Green's function tensor components, so the empirical Green's functions used here refer to the sum of the tensor components as recorded on three component seismograms. Thus, exact modeling for different focal mechanism solutions of these earthquakes is not possible, so the source events used for empirical Green's functions should have nearly the same focal mechanism as the elemental source. Hutchings (1987) found that when focal mechanism solutions differ by less than $15^{\circ}$ in either strike, dip, or slip vector, very similar waveforms are preserved for frequencies up to $10 \mathrm{~Hz}$ and spatial separations up to $3.0 \mathrm{~km}$, depending on the recording site's geology. However, the dominating effect of recording site geology ensures 
that a consistent frequency content, amplification, and energy distribution is recorded from elemental sources reguardless of their focal mechanism or hypocenter. Empirical Green's functions can then be normalized and used as site-response functions.

\section{Theory}

No earthquake has an actual step-impulsive point source, but if the source is small enough, the rupture duration is too short to affect seismograms. The only effect on seismograms, then, is linear amplification from the scalar seismic moment and the radiation pattern of the focal mechanism solution. From the representation relation (Aki and Richards, 1980), this is expressed as (Hutchings, 1987)

$$
e_{n}(X, t)=M_{p q} H\left(t^{\prime}\right) * G_{n p, q}\left(X^{\prime}, t^{\prime} ; X, t\right),
$$

where $M_{p q}$ is the scalar moment tensor, $H\left(t^{\prime}\right)$ is the step source time function, and $G_{n p}$ is the elastodynamic Green's function. Primed terms refer to descriptions of the source.

Unfortunately, a further decomposition of the seismograms to obtain individual Green's function tensor components is not possible. If the Cartesian coordinates are rotated so that $X=\left(x_{1}, x_{2}, x_{3}\right)$ is in the radial, tangential, and vertical direction, several spatial derivatives of the Green's function tensor components are equal to zero (Johnson, 1974). Then, the time derivative of Equation 1, for a double-couple source, can be expressed as: 
(2)

$$
\begin{aligned}
& \dot{e}_{1}(X, t)=M_{o}\left\{B_{1}\left(G_{11,1}-G_{12,2}\right)-B_{2}\left(G_{11,3}+G_{13,1}\right)+B_{3}\left(.5 G_{11,1}+.5 G_{12,2}-G_{13,3}\right)\right\} \\
& \dot{e}_{2}(X, t)=M_{o}\left\{B_{4}\left(G_{21,2}+G_{22,1}\right)-B_{5}\left(G_{23,2}+G_{22,3}\right\}\right. \\
& \dot{e}_{3}(X, t)=M_{o}\left\{B_{1}\left(G_{31,1}-G_{32,2}\right)-B_{2}\left(G_{31,3}+G_{33,1}\right)+B_{3}\left(.5 G_{31,1}+.5 G_{32,2}-G_{33,3}\right)\right\}
\end{aligned}
$$

for the constants $B_{1}$ through $B_{5}$,

$$
\begin{aligned}
& B_{1}=-\sin \delta \cos \lambda \sin 2 \phi+.5 \sin 2 \delta \sin \lambda \cos 2 \phi \\
& B_{2}=\cos 2 \delta \sin \lambda \sin \phi+\cos \delta \cos \lambda \cos \phi \\
& B_{3}=-\sin 2 \delta \sin \lambda \\
& B_{4}=\sin \delta \cos \lambda \cos 2 \phi+.5 \sin 2 \delta \sin \lambda \sin 2 \phi \\
& B_{5}=-\cos 2 \delta \sin \lambda \cos \phi+\cos \delta \cos \lambda \sin \phi,
\end{aligned}
$$

are obtained from the fault geometry and slip vector description for $\lambda, \delta$, and $\phi$ of Aki and Richards (1980, sec. 4.5). The constant $B_{1}$ through $B_{5}$ along with the three recorded components of the recorded seismograms are insufficient to solve the set of linear equations for the fourteen Green's functions tensor components. Several events at the same location, with different focal mechanism solutions, would have to be recorded to determine all the Green's functions components for a particular source-station pair. For purposes of obtaining Green's functions from actual recordings, only the summed effect of Green's functions tensor components can be obtained. This means that displacement from different focal mechanism solutions cannot be explicitly obtained. 
When empirical Green's functions are not obtained for all possible elemental source locations, an approximation must be made. From Hutchings (1987), the empirical Green's function for the elemental source can be approximated as

$$
e_{n}(X, \omega)_{i} \cong \frac{\bar{X}_{o} B_{i}}{\bar{X}_{i} B_{o}} \exp \left(\frac{\omega\left(\bar{X}_{o}-\bar{X}_{i}\right)}{2 c Q}\right) e_{n}\left(X_{o} ; X, \omega\right)
$$

where $\bar{X}$ is hypocentral distance, and $B$ is the radiation coefficient; subscripts $i$ and $o$ refer values for the elemental source and the source event, respectively. $\omega$ is angular frequency, $c$ is seismic velocity of the structure, and $Q$ is the seismic quality quotient. For near-source seismograms, secondary arrivals result from transmission from many incident orientations, and the focal mechanism correction factor is inappropriate for the entire wavetrain. Hutchings (1987) suggests that the correction factor be restricted to $-0.25 \leq$ $B_{o} \leq 0.25$ as a reasonable approximation of the effect of the focal mechanism on the entire empirical Green's function. Hutchings showed that empirical Green's functions corrected in this way are consistent in amplitude, frequency content, and energy distribution even for source events located up to $20.0 \mathrm{~km}$ apart. Empirical Green's functions, then, represent site response functions.

Empirical Green's functions can be combined with synthetic slip functions, and summed to synthesize an extended source earthquake. Because their source events have spatial extent, they can be summed to simulate fault rupture without loss of information, thereby potentially exactly computing the representation relation.

This is expressed as

$$
u_{n}(X, t)=\sum_{i} \frac{\mu A_{i} S\left(t_{i}^{\prime}\right)_{i}}{M_{o i}} * e_{n}\left(X, t_{i}^{\prime}-\frac{\bar{\chi}_{i}}{v}\right)_{i}
$$


Here, $S\left(t_{i}^{\prime}\right)_{i}$ is the desired slip function analytically deconvolved with the step function, and $t_{i}^{\prime}$ is relative to origin time of source event corrected for element location. $e_{n}\left(X, t_{i}^{\prime}\right)_{i}$ is the recorded displacement from the small earthquake used as an elemental source for the

$i^{\text {th }}$ element. $\bar{\chi}_{i}$ is the radial distance from the hypocenter to the elemental source, and $v$ is rupture velocity. $M_{o i}$ is the static seismic moment of the empirical Green's function. $A_{i}$ is an elemental area such that $\sum A_{i}$ equals total area, and $\mu$ is rigidity. Since empirical Green's functions scale linearly only in amplitude with differences in seismic moment, they can provide the contribution from any appropriate discretized elemental source area.

\section{Computation of Synthetic Seismograms}

Program EMPSYN is a seismogram synthesis program that utilizes empirical Green's functions. A discretized algorithum of the representation relation using empirical Green's functions is programmed in FORTRAN 77 (Appendix B). Appendix A provides an example of using Empsyn to synthesize the observed ground motion from the 1971 San Fernando earthquake.

In EMPSYN equation 5 is computed in the time domain by (Hutchings, 1987):

$$
u_{n}(X, t)=\sum_{i} \sum_{j} \frac{\mu A_{i} \bar{s}_{j}}{M_{o i}} e_{n}\left(X, t_{i}^{\prime}-\frac{\chi_{i}}{v}-j \Delta t^{\prime}\right)_{i}
$$

where $t_{i}^{\prime}$ is relative to the origin time of the empirical Green's function corrected for location of the elemental source. The summation $j$ follows the desired slip function at an element, and the summation over $i$ follows the rupture front. In the frequency domain Equation 5 is computed by:

$$
u_{n}(X, \omega)=\sum_{i} \frac{\mu A_{i} S(\omega)_{i} \bar{X}_{o} B_{i}}{M_{o i} \bar{X}_{i} B_{o}} \exp \left(\frac{\omega\left(\bar{X}_{o}-\bar{X}_{i}\right)}{2 c Q}\right) \exp \left(\frac{-\chi_{i}}{v}\right) e_{n}\left(X_{o} ; X, \omega\right)
$$


The second exponential is phase shift due to time delays for the rupture front. It is apparent that Equation 7 is equal to Equation 5 if the elemental source and source event have the same location. If no source event has a location near elemental sources, the computation is equivalent to that with only site response functions. Equation 7 then becomes a synthetic solution for a homogeneous whole space convolved with a site response function.

Computationally EMPSYN first sets up an array of source elements for any specified fault rupture surface shape. Each element is assigned the empirical Green's function from the closest source event. Appropriate time shifts are applied to the empirical Green's function to account for the difference in distances between the actual event and the elemental source location. This correction is already incorporated in the $t_{o i}$ in Equation 6 and as a phase shift for $e_{n}(X, \omega)$ in Equation 7. An additional time delay is applied for the rupture propagation as $\frac{\chi_{i}}{v}$ in Equation 6 and an additional phase shift is Equation 7. An additional time delay or phase shift is added to the S-wave portion of empirical Green's functions to account for adjustments to S-P time. Radiation correction factors are applied to the empirical Green's that account for the source-station geometry from each elemental source location. $\mathrm{P}$-wave radiation correction factor is applied to the portion of seismograms up to about $0.5 \mathrm{~s}$ before the S-arrival. SV-wave correction factors are applied to the remaining portion of the vertical and radial components, and the SH-wave correction factor is applied to the remaining portion of the tangential component. An option not to include a radiation correction factors is available. Computations with the time domain are only for exact synthesis and do not include attenuation and geometrical spreading corrections, but do include mechanism correction factors.

The discretized source area is limited by the source dimension for small earthquakes that provide empirical Green's functions. Using the Brune (1970) source model, the largest source area for events providing empirical Green's functions for a moment of $1.0 \times 10^{21}$ dyne-cm (used in Hutchings, 1987) is about $0.1 \mathrm{~km}^{2}$. However, care must be taken that the radially symetric source elements have center points with distances less than about 
$0.15 \mathrm{~km}$ to ensure frequency integrity below $15 \mathrm{~Hz}$. This corresponds to elemental source areas of about $0.05 \mathrm{~km}^{2}$.

The Kostrov slip model (Kostrov, 1964; Buridge and Willis, 1969), a Kostrov-ramp, and the two-dimensional Haskel model (Dahlen, 1974; Savage, 1972) with both step and ramp displacement are used by EMPSYN. Surface rupture is modeled by terminating synthesis at the free surface, with slip at the surface being that originally specified for the surface element. For example, a circular fault rupture with the Kostrov slip function that encounters the free surface will have displacement at the surface element as if the fault extended to the originally specified rupture area.

The Kostrov slip model is an exact solution for a circular crack propagation in a homogeneous medium

$$
S\left(X^{\prime}, t^{\prime}\right)=\frac{c \sigma \beta}{\mu}\left(t^{\prime 2}-\frac{\chi_{i}^{2}}{V_{r}^{2}}\right)^{\frac{1}{2}} H\left(t^{\prime}-\frac{\chi_{i}}{V_{r}}\right),
$$

where $\chi_{i}$ is position vector from hypocenter, $t^{\prime}$ is relative to origin time of the earthquake, $V_{r}$ is rupture velocity, $\beta$ is shear wave velocity, $c$ is a constant equal to 0.81 for $V_{r}$ equal to $0.9 \beta, \mu$ is rigidity assumed equal to $3.0 \cdot 10^{11}$, and $\sigma$ is stress drop.

In EMPSYN the Kostrov slip function is time shifted to element time:

$$
t_{i}^{\prime}=t^{\prime}-\frac{\chi_{i}}{V_{r}}
$$

Then the Kostrov slip function for time relative to rupture at the element is

$$
S\left(t_{i}^{\prime}\right)_{i}=\frac{0.81 \sigma \beta}{\mu}\left(t_{i}^{\prime 2}+\frac{2 \bar{\chi}_{i} t_{i}^{\prime}}{V_{r}}\right)^{\frac{1}{2}} H\left(t^{\prime}\right)
$$


where $t_{i}^{\prime}$ goes from 0 to the rise time $\tau_{i}$. $\beta$ is shear wave velocity and is calculated from the velocity model.

EMPSYN uses Equation 9 directly for time-domain computations (Equation 6), where $\mathrm{H}\left(\mathrm{t}^{\prime}\right)$ is provided by the slip function of the empirical Green's function. For frequency domain computations, EMPSYN uses a ramp slip function deconvolved with a step function (described below), with displacement at each element designated by the Kostov slip function. Both the Kostrov and Kostrov-ramp models have rise time at an element determined by the time from the arrival time of the rupture front until the rupture front reaches the closest fault edge and a "healing phase" travels back to the element at the P- or S-wave velocity.

The healing velocity is calculated from the rupture velocity: $V_{h}=V_{r} \times 1.76 / 0.9$. The rise time is then

$$
\tau_{i}=\frac{X_{o i}^{\prime}}{V_{r}}+\frac{\left(X_{o i}^{\prime}-\chi_{i}\right)}{V_{h}}
$$

where $X_{o i}$ is the closest point on the rupture perimeter to the element. Then,

$$
\tau_{i}=\left(\chi_{i}-X_{i}^{\prime}\right)\left(\frac{1}{V_{R}}+\frac{1}{\alpha}\right)
$$

The Haskel models have the same fault displacement for all elements, and only the rise time and rupture velocity are varied.

The ramp slip function, for both the Kostrov-ramp and Haskel models, is applied only in the spectral domain. It is the sum of a positive and negative infinite ramp displaced by the rise time. The slope ASLP is equal to the desired amplitude divided by the rise-time. 
The infinite ramp can be expressed as the integral of the step function. In the frequency domain

$$
\begin{aligned}
R(\omega) & =\frac{-i \pi A S L P \delta(\omega)}{\omega}, \quad \omega=0 \\
& =\frac{-A S L P}{\omega^{2}}, \quad \omega \neq 0 .
\end{aligned}
$$

The negative ramp then is

$$
R^{\prime}(\omega)=-R(\omega) e^{-i \omega \tau_{o}}
$$

where $\tau_{0}$ is the rise time. The slip function used in EMPSYN is the ramp deconvolved by the unit step function

$$
\begin{aligned}
S(\omega) & =A S L P\left(\frac{-i}{\omega}+\frac{i e^{-i \omega \tau_{o}}}{\omega}\right), \quad \omega \neq 0 \\
& =0, \quad \omega=0 .
\end{aligned}
$$

For the Haskel slip models ASLP is the same for each element; for the Kostrov-ramp it varies as described above.

\section{Program EMPSYN Documentation}

\section{Input Parameters}

Two input files and empirical Green's functions data files are necessary:

FAULT- provides an array of distances from the hypocenter to the edge of the fault rupture area at equal angles. Number of values on first line (IP) and distances in free 
format stating on line 2 (FL(I)). If the number of distances is set at 1 , then the distance is the radius of a circular fault, and 300 radial distances are created for computations. If IP is set to 3 then an elliptical fault area is used and and values on line 2 are the eccentricity, length of major axis and the rake of the major axis from the hypocenter. An array of $\mathbf{3 0 0}$ fault distances from the hypocenter to the ellipse edge is calculated (updip first, clockwise for others) for use in the program. If IP is 4 , then a rectangular fault with distances from the hypocenter to the four sides specified by the distances is setup with 300 radial distances.

SOURCES- a list of source events, their location, moment, focal mechanism solution, and a time to split $\mathrm{P}$ - and S-wave portion of their seismograms. Values are listed in free format on a single line for each event. Variables in order are: EAA, EBB, SPP, BLAT, BLON, BH, BM, BSTR, BDP, BSV for days, hours-mins, split time, latitude, longitude, depth, moment $\mathrm{w} / \mathrm{o}$ factor $10^{20}$, strike, dip, and slip vector respectively. Focal mechanism solutions are described as convention of Aki and Richard (1980).

EMPIRICAL GREEN'S FUNCTIONS Time series used for empirical Green's functions stored in either SAC (LLNL, Seismic Analysis Code) or formated ASCII files. The three component files have been rotated into radial, tangential ( $90^{\circ}$ clockwise from radial), and vertical (up for compression) components relative to the source event. The first point of the data file has time relative to origin time of the source event.

Other Input values (input from terminal)

STK,DP,SV: strike, dip, slip vector of event to be modeled.

MO: moment of modeled event in dyne-cm. This is used only for the Haskel rupture models.

ELA: area of elemental sources in $\mathrm{km}^{2}$. 
STD: stress drop in Bars. This is only used for Kostrov slip functions.

VR: rupture velocity in $\mathrm{km} / \mathrm{s}$.

VD: $P$ velocity at $10.0 \mathrm{~km}$ for determining velocity gradient VPP. $\quad$ VPP $=(\mathrm{VD}-3.0) / 10.0$

VP: velocity for travel time correction due to difference in location of element and source event. VP set for each source event: $\quad \mathrm{VP}=3.0+\mathrm{BH} 1 * \mathrm{VPP}$

ALAT, ALON, AH: Hypocenter location of event to be modeled in deg and decmil degrees.

SB: two-digit identification of station for synthesized seismogram corresponding to the station where empirical Green's functions recorded,

SLAT, SLON: location of station SB.

RTMHSK: Rise time for Haskel-ramp slip function.

\section{Source elements}

Source elements are in concentric rings from hypocenter, divided into equal area segments. The thickness and arc length of each element is determined by finding an integer multiple number of elements of specified source area with arc length closest to ring width. Each element is checked to determine whether the center of the element is outside the rupture perimeter. The elements are assigned a sequential number (KK). Rings are added until no elements are assigned to the last ring.

Figure 1 shows output for a synthesis using the Kostrov-ramp slip function. Following the printout of input parameters is the description of elements. The number of elements for each ring is listed for the specified fault parameters. Individual element parameters are listed for ring 3 (elements 9 through 22). ANG4 is the angle in the fault plane, from $0.0^{\circ}$ 
up dip from the hypocenter to the center of the element. ANG5 is the angle to the element in the horizontal plane. AZMM is the azimuth from north for the element, followed by the event identification. DIS is hypocentral distance from the source event to the element. DTIMP is the travel time delay for the empirical Green's function due to the difference between the element to the station and the source event location and the element. RDPP is the total displacement for the element. RISTM is the rise time of the element. Here they are equal since. it is circular rupture.

\section{Rotation and Correction for Focal Mechanism Solution}

Figures 2 and 3 show the plot and printout of a unit impulse seismogram that has been rotated from (UP,320.4,050.4) for $\mathrm{Z}, \mathrm{R}, \mathrm{T}$ to $(\mathrm{UP}, 000,090)$, and whose focal mechanism solution has been corrected from $270,045,+90$ for (STK,DP,SV) to $180,090,+00$. The $\mathrm{P}, \mathrm{SV}, \mathrm{SH}$ correction factors are

$$
F F P P=0.4 ; \quad F F S V=-0.4 ; \quad F F S H=-0.4
$$

for the focal mechanism solution of the source event, and

$$
E L P P=-0.511 ; \quad E L S V=-0.491 ; \quad E L S H=0.4
$$

for the element. Then the P- and S-wave amplitudes on the vertical trace are

$$
P_{a m p}=1.0(E L P P / F F P P)=-1.28 ; \quad S_{a m p}=1.0(E L S V / F F S V)=1.23
$$

The $\mathrm{P}$ and S-wave amplitude on the $000^{\circ}$ component are

$$
\begin{gathered}
P_{a m p}=E L P P / F F P P(\cos 320+\cos 50)=-1.80 \\
S_{a m p}=E L S V / F F S V \cos 320+E L S H / F F S H \cos 50=0.30 .
\end{gathered}
$$


The $\mathrm{P}$ and S-wave amplitudes on the $090^{\circ}$ component are

$$
P_{a m p}=E L P P / F F P P(\sin 320+\sin 50)=-0.17
$$

$$
S_{a m p}=E L S V / F F S V \sin 320+E L S H / F F S H \sin 50=-1.55
$$

If the element and the source event had different locations, initially there would be a rotation of the source event to the radial and tangential components of the element before these corrections were applied. 


\section{Test Runs}

To test the slip functions consider a single element circular fault model with radius $0.1 \mathrm{~km}$, and a unit Dirac delta function "empirical Green's function." The P and S pulses have width $0.01 \mathrm{~s}$, equal to the digitizing rate, and model a far-field displacement seismogram.

Figures 4 through 7 show the plot and printout of synthesized seismograms for the Haskelstep (HSKS), Haskel-ramp (HSKR) with rise time of .001, Kostrov-ramp (KSR) with rise time of .001 , and the Kostrov (KOS) with rise time of .001 second. Here, the source event and synthesized event have the same hypocenter, focal mechanism solution, and moment; and, the empirical Green's function and the synthesized seismogram have the same orientation. That all seismograms are exactly equal shows that correct computations for several factors: geometrical spreading and $Q$ due to differences in location of the source event and element for the spectral domain computation; rotation due to differences in orientation of station to source event and station to element; and corrections for differences in radiation pattern due to focal mechanism solutions, and; correction factors for adjusting element contribution due to differences in desired moment and that obtained by incremental stacking (FAC1, FAC2) for time-domain computations. In addition, the fact that the time difference between the $\mathrm{P}$ and $\mathrm{S}$ pulse is identical shows that the splitting of the seismograms for spectral domain processing, and reconnection for the synthesis are done correctly.

Figures 8 through 10 show synthesized seismograms for a point source (a single element is specified, but the center point of the element is used for rupture propagation calculations) with rise time $1.0 \mathrm{~s}$ for the same orientations and moment as the source event, using the Haskel-ramp, Kostrov-ramp, and Kostrov slip functions. The function appear as boxcars because the slip functions are deconvolved with the step function (Equation 14), which results in a differentiation of the slip function. The Kostrov time domain slip function is a ramp slip function in this case because $\chi_{i}$ in Equation 9 is zero, which results in a 
ramp function. Since the source event and synthesized seismogram have the same moment, the integral of their pulses should be the same: $0.01 \mathrm{~cm}-\mathrm{sec}$. This remains constant since the source event has amplitude 1.0 and width .01 , and the synthesized seismogram has amplitude 0.01 and width $1.0 \mathrm{~s}$. I have no explanation for the negative DC offset of the spectral computations of Haskel-Ramp and Kostrov-ramp slip functions. These tests confirm the basic workings of the slip function computations.

Figures 11 through 14 show synthesized seismograms for a circular fault of radius 1.0 $\mathrm{km}$ and moment $1.0 \times 10^{23}$ dyne-cm, and synthesis using the Haskel-step, Haskel-ramp, Kostrov-step, and Kostrov slip functions. The elemental source size is $0.01 \mathrm{~km}^{2}$ and the focal mechanism solution is the same as the source event. The shapes and durations of the synthesized seismograms are different, but the areas under the pulses are equal. The finite dimensions of the fault result in a variation in rotation and radiation correction factors and a diminished amplitude of S-waves relative to P-waves.

Synthesized seismograms for an extended source with an irregular fault shape and asymetric rupture greatly affect seismograms. Here, a synthesis is done using computed "empirical Green's functions" from a half-space Green's function computation (Johnson, 1975). Pand S-wave velocities are 4.5 and $2.55 \mathrm{~km} / \mathrm{s}$, and density is $2.2 \mathrm{gm} / \mathrm{cm}^{2}$. Table 1 shows the location parameters for the synthesized "empirical Green's functions." They are computed for fault orientation STK,DP,SV $(090,090,00)$ and a moment of $1.0 \times 10^{20}$ dyne-cm. Table 2 shows the location parameters for the stations. Figures 15, 16, and 17 show the vertical, radial $\left(320.4^{\circ}\right)$, and tangential $\left(50.4^{\circ}\right)$ components for the synthesized "empirical Green's functions" at station 01 . 
Table 1

Source Events Parameters

$\begin{array}{llll}\text { Station I.D. } & \text { Latitude }\left({ }^{\circ} \mathrm{W}\right) & \text { Longitude }\left({ }^{\circ} \mathrm{N}\right) & \text { Depth }(\mathrm{km}) \\ 0000001 & 34.5000 & 118.5437 & 1.0 \\ 0000002 & 34.5000 & 118.5220 & 1.0 \\ 0000003 & 34.5000 & 118.5000 & 1.0 \\ 0000004 & 34.5000 & 118.4780 & 1.0 \\ 0000005 & 34.5000 & 118.4563 & 1.0 \\ 0000006 & 34.5000 & 118.5437 & 3.0 \\ 0000007 & 34.5000 & 118.5220 & 3.0 \\ 0000008 & 34.5000 & 118.5000 & 3.0 \\ 0000009 & 34.5000 & 118.4780 & 3.0 \\ 0000010 & 34.5000 & 118.4563 & 3.0 \\ 0000011 & 34.5000 & 118.5437 & 5.0 \\ 0000012 & 34.5000 & 118.5220 & 5.0 \\ 0000013 & 34.5000 & 118.5000 & 5.0 \\ 0000014 & 34.5000 & 118.4780 & 5.0 \\ 0000015 & 34.5000 & 118.4563 & 5.0\end{array}$

Table 2

Stations for Seismogram Synthesis

$\begin{array}{lll}\text { Station I.D. } & \text { Latitude }\left({ }^{\circ} \mathrm{N}\right) & \text { Longitude }\left({ }^{\circ} \mathrm{W}\right) \\ 01 & 34.5451^{\circ} & 118.5000^{\circ} \\ 02 & 34.5000^{\circ} & 118.3911^{\circ} \\ 03 & 34.4549^{\circ} & 118.5000^{\circ} \\ 04 & 34.5000^{\circ} & 118.6089^{\circ}\end{array}$

The extended source being modeled has dimensions $6 \times 10 \mathrm{~km}$, Moment: $1.0 x 10^{25} \mathrm{dyne} / \mathrm{cm}$, Strike: $N 090^{\circ} \mathrm{E}, \mathrm{Dip}: 90^{\circ} \mathrm{N}$, and Slip vector: $0^{\circ}$. Figure 18 shows the synthesized seismogram for hypoocenter located at the center of the fault: $34.5000^{\circ} \mathrm{N}, 118.5000^{\circ} \mathrm{W}$, depth $3.0 \mathrm{~km}$. The discrete pulses in the synthesis is a result of the elemental size being large enough that the delay time between elements is greater than the source event pulse widths. Figure 19 shows extended output for all the 1,207 elements used in the computation: for each element, the angle from the hypocenter in fault plane (ANG4), angle and azimuth in 
the horizontal plane (ANG5, AZMM), identification of source event assigned (here they print out as 01 through 0 15), the distance from the element to the source event (DIS), the delay time added to the elemental source due to the difference in location (DTIMP), and the location of the element.

For the first five rings (ring identification is listed after the element information), the same source event is used for all the elements. The distance from source event to the element is the same as the radius of the ring of elements, since the source event has the same location as the hypocenter of fault rupture. Starting with ring 6 , different source events are appropriate for some elements and DIS varies from the ring radius. 


\section{SYNTHESIS OF EVENT ODOO AT STATION $\theta$}

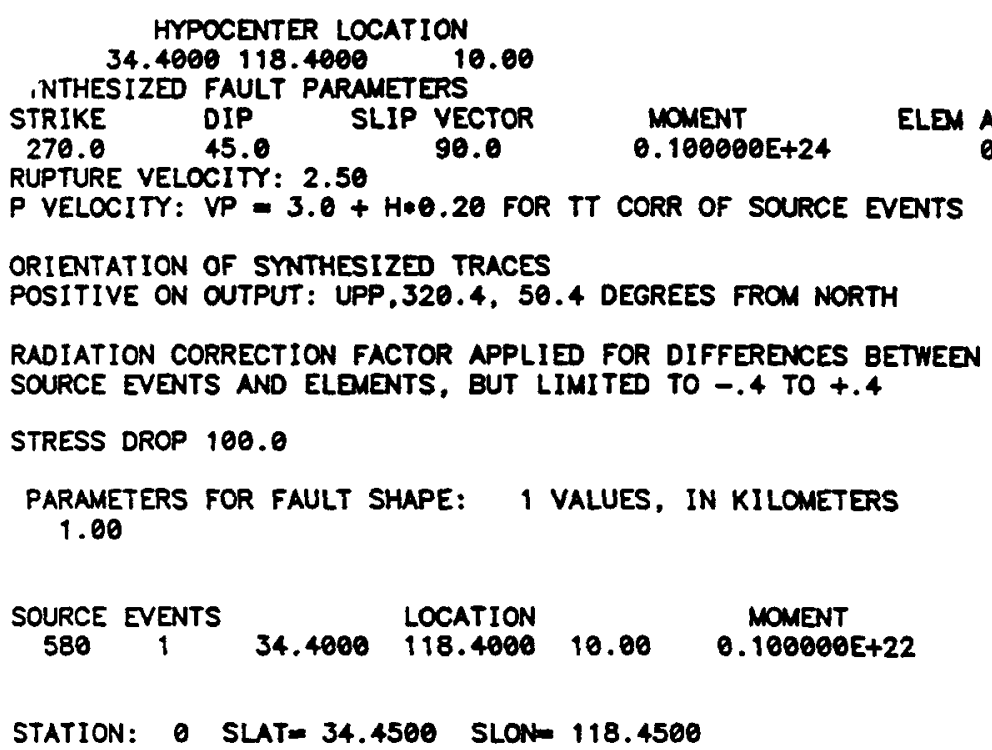


ROTATE AND CORRECT FOR FOCAL MECHANISM

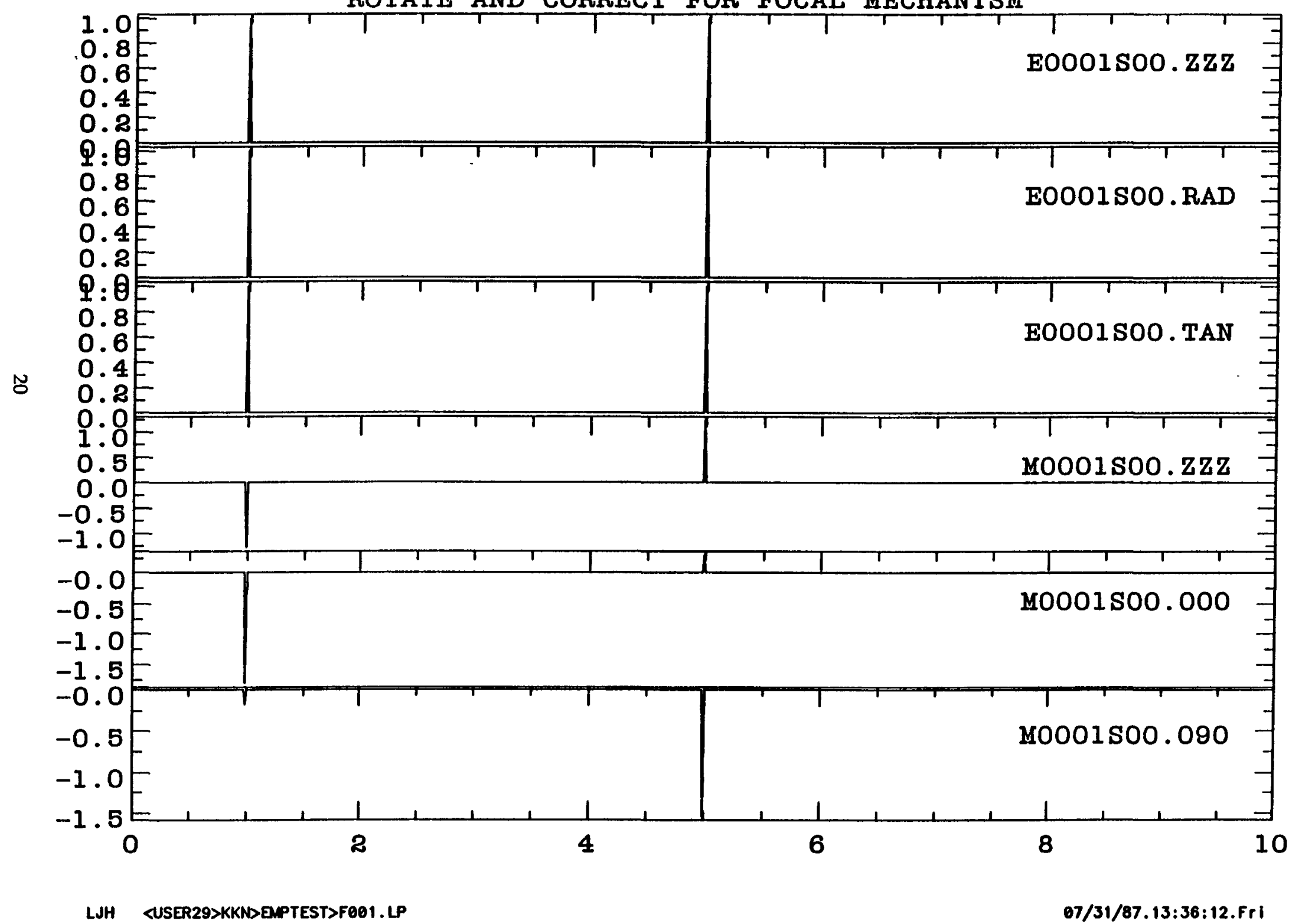

Sinn 1 
SYNTHESIS OF EVENT QOOI AT STATION 0

HYPOCENTER LOCATION

$34.4000 \quad 118.4000 \quad 10.00$

INTHESIZED FAULT PARAMETERS

STRIKE DIP SLIP VECTOR

$\begin{array}{lll}180.0 & 90.6 & 0.0\end{array}$

RUPTURE VELOCITY: 2.50

$P$ VELOCITY: VP $=3.0+H * 0.28$ FOR TT CORR OF SOURCE EVENTS

ORIENTATION OF SYNTHESIZED TRACES

POSITIVE ON OUTPUT: UPP, $0.0,90.0$ DEGREES FROM NORTH

RADIATION CORRECTION FACTOR APPLIED FOR DIFFERENCES BETWEEN

SOURCE EVENTS AND ELEMENTS. BUT LIMITED TO -.4 TO +..4

PARAMETERS FOR FAULT SHAPE: i VALUES, IN KILOMETERS 0.10

SOURCE EVENTS

LOCATION

$34.4000 \quad 118.4000 \quad 10.00$

MOMENT

$0.100000 E+22$

STATION: O SLAT $=34.4500$ SLON 118.4500

TOTAL NUMBER OF ELEMENTAL AREAS IS 1 MAXIMUM RADIUS $0.13 \mathrm{kM}$

THO DIMENSIONAL HASKEL RUPTURE MODEL WITH STEP SLIP FUNCTION

EACH ELEMENT HAS THE SAME DISPLACEMENT: $6.7 \mathrm{CM}$

TOTAL FAULTING DURATION 0.04 SECONDS

SLIP FUNCTION INCREMENTED BY DIGITIZING RATE

SOURCE EVENTS AND SYNTHESIZED OUTPUT LIMITED TO 1000 POINTS

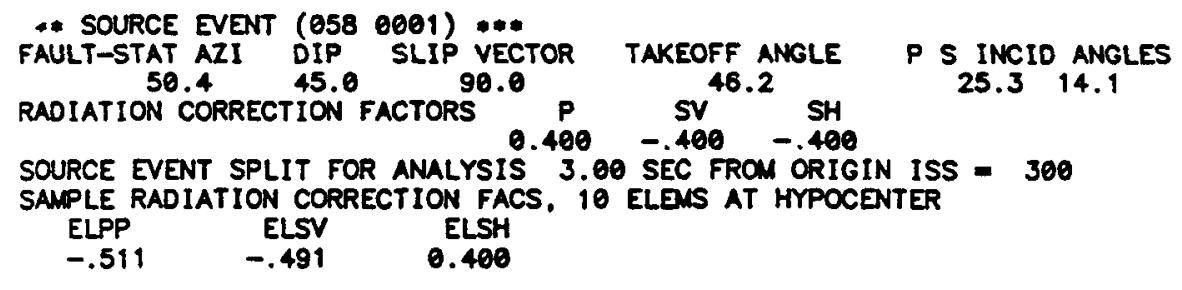

MOMENT OF ACTUAL EVENT $0.100000 E+22$

MOMENT OF MODELED EVENT $0.100000 E+22$ 
HASKEL STET FUNCTION

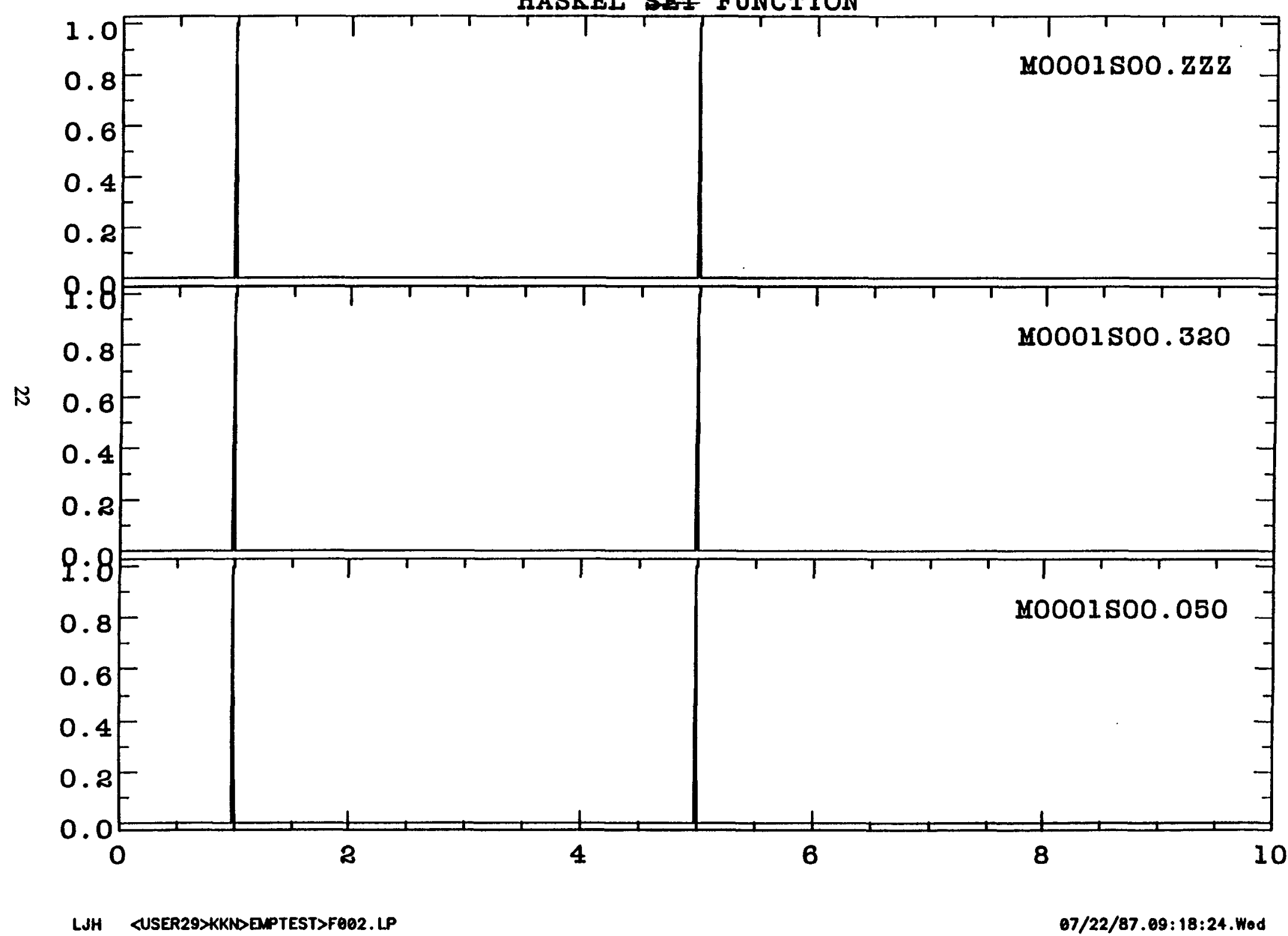

E:.... 
SYNTHESIS OF EVENT OOOI AT STATION

$$
\text { Sigure } 4
$$

HYPOCENTER LOCATION

$34.4000 \quad 118.4000 \quad 10.00$

NTHESIZED FAULT PARAMETERS

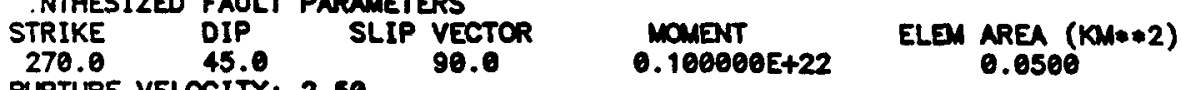

RUPTURE VELOCITY: 2.50

P VELOCITY: VP $=3.0+$ H*0.20 FOR TT CORR OF SOURCE EVENTS

ORIENTATION OF SNNTHESIZED TRACES

POSITIVE ON OUTPUT: UPP,320.4, 50.4 DECREES FROM NORTH

RADIATION CORRECTION FACTOR APPLIED FOR DIFFERENCES BETWEEN

SOURCE EVENTS AND ELEMENTS, BUT LIMITED TO -.4 TO +.4

$\underset{\substack{\text { PARAMETERS FOR FAULT SHAPE: } \\ 0.10}}{1 \text { VALUES, IN KILOMETERS }}$

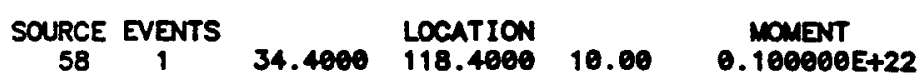

STATION: $\theta$ SLAT= 34.4500 SLON 118.4500

TOTAL NUMBR OF ELEMETTAL AREAS IS 1 MAXIMM RADIUS $0.13 \mathrm{kM}$

TWO DIMENSIONAL HASKEL RUPTURE MODEL WITH STEP SLIP FUNCTION

EACH ELEMENT HAS THE SAME DISPLACEMENT: $6.7 \mathrm{cM}$

TOTAL FAULTING DURATION 0.04 SECONDS

SLIP FUNCTION INCREMENTED BY DIGITIZING RATE

SOURCE EVENTS AND STNTHESIZED OUTPUT LIMITED TO 2000 POINTS

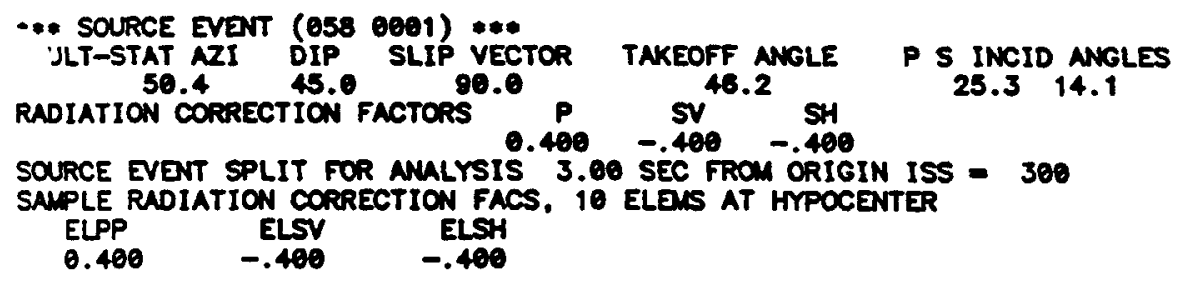

MOMENT OF ACTUAL EVENT $0.100000 E+22$

MOMENT OF MODELED EVENT $0.100000 E+22$ 


\section{HASKEL RAMP SLIP FUNCTION}

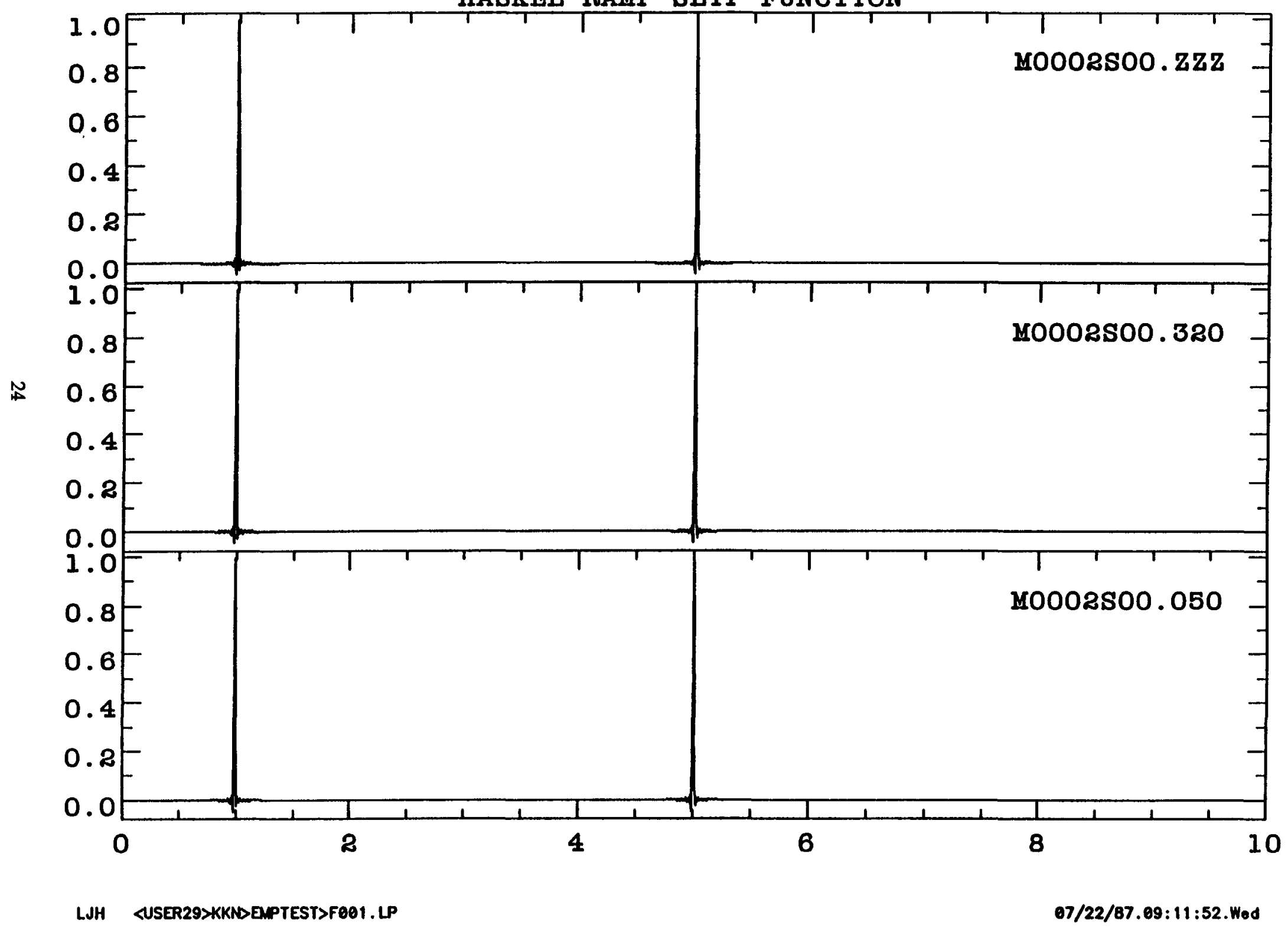

ficules 
SYNTHESIS OF EVENT OOOZ AT STATION 0

$$
\text { figure } 5
$$

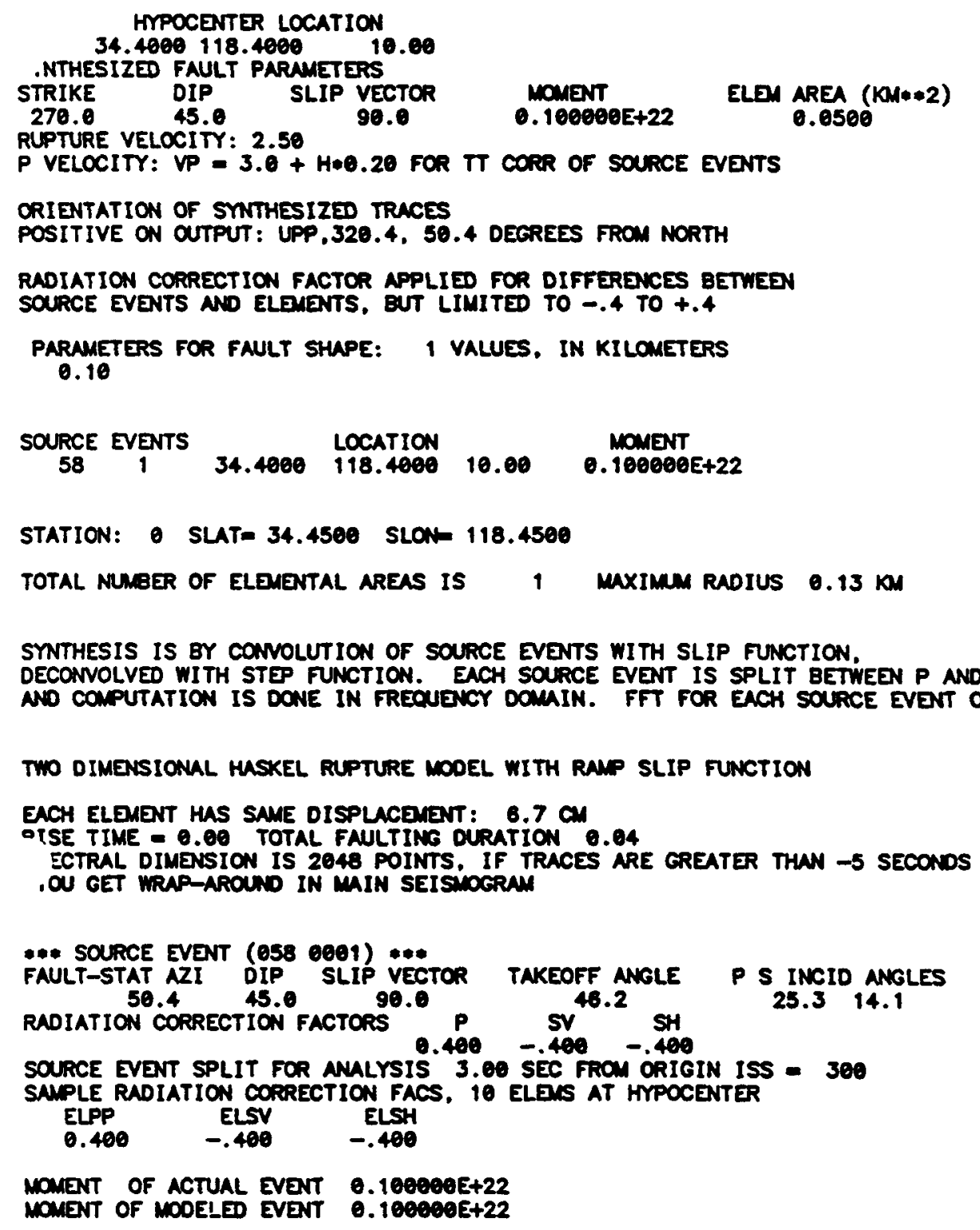


MODIFIED KOSTROV SLIP FUNCTION

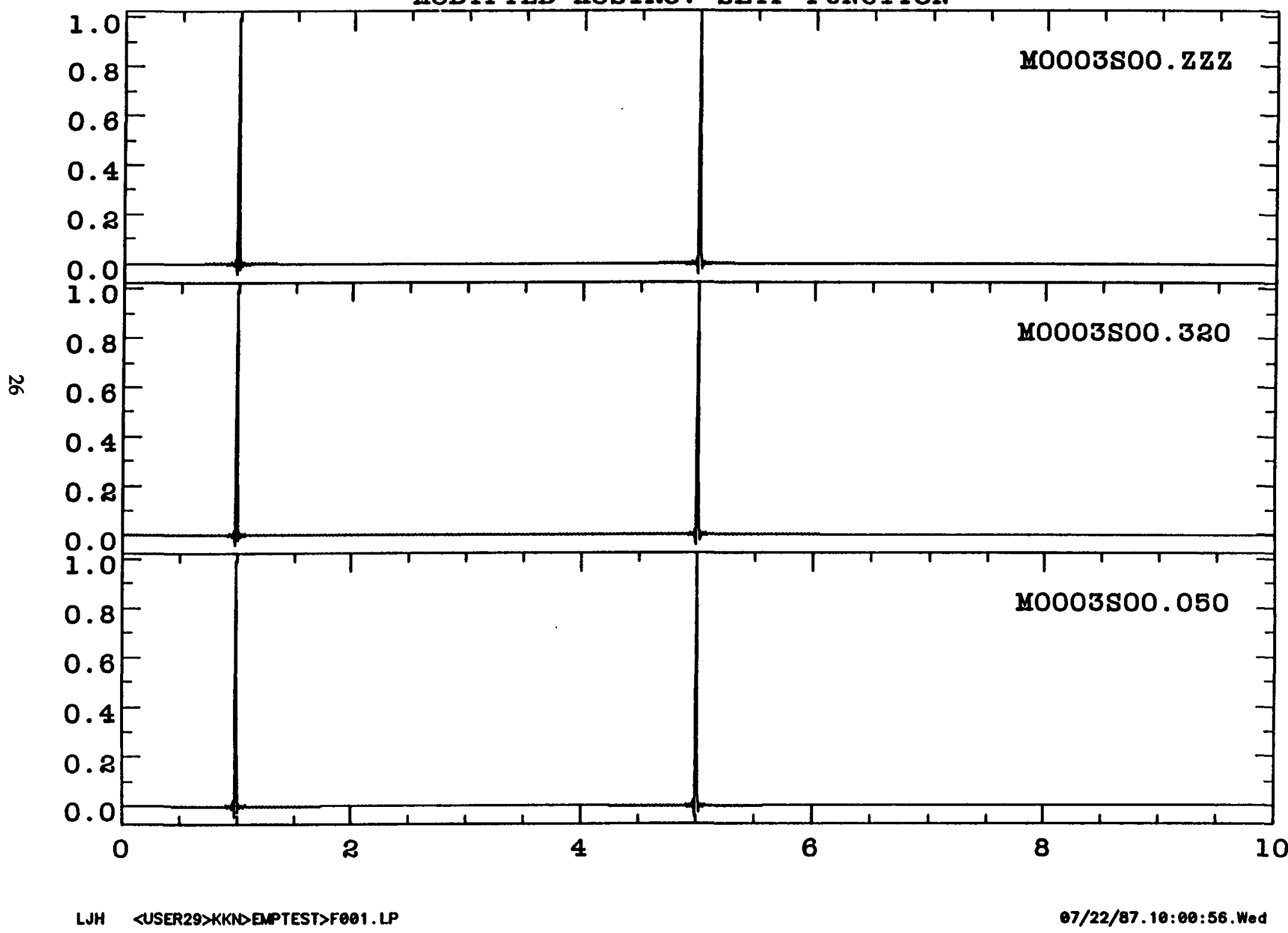


SWNTHESIS OF EVENT 0003 AT STATION 0
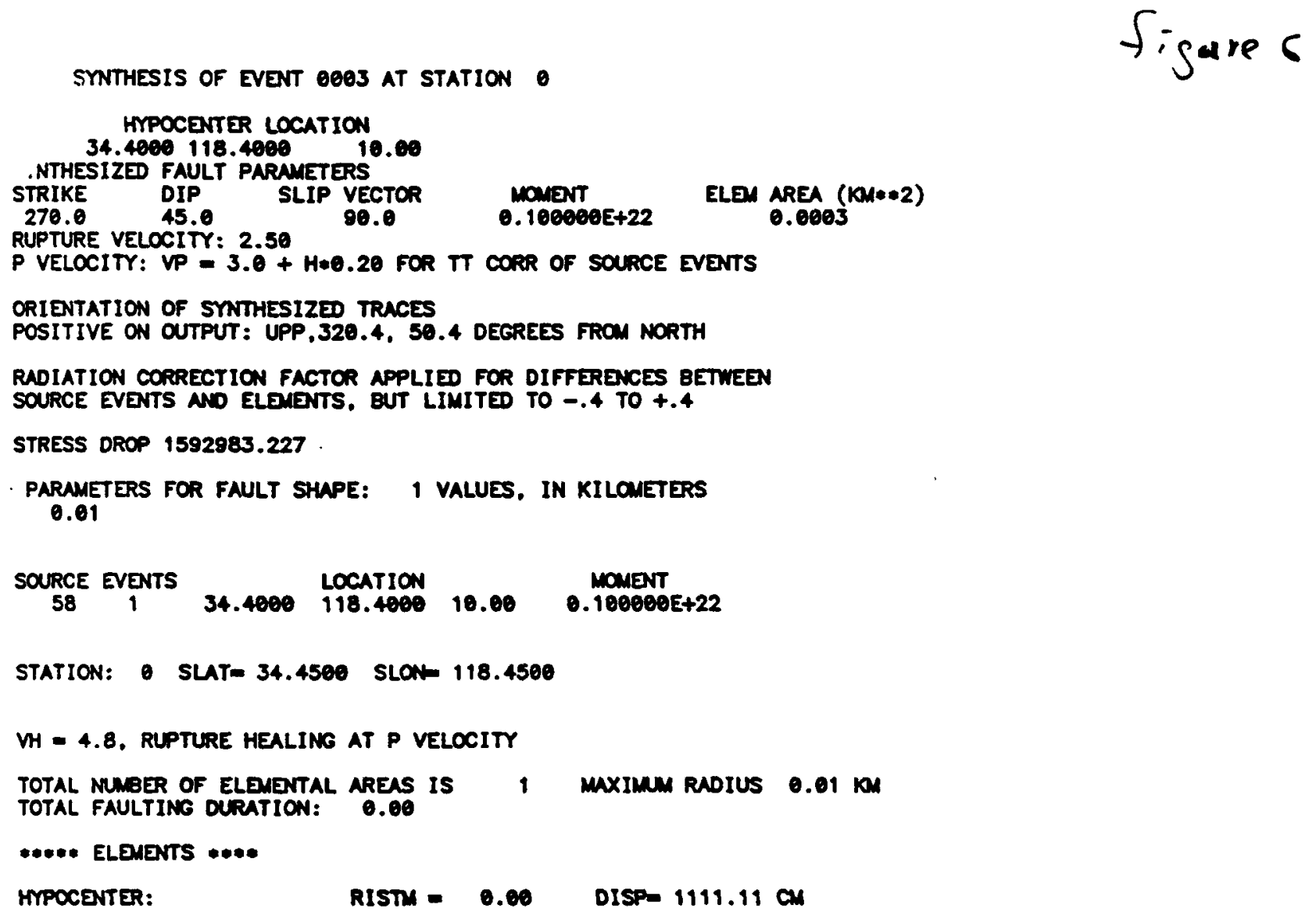

SLIP APLITUDE AND RISE TIME CONSTRAINED EY SELF-SIMILAR KOSTRON SLIP. MULTIPLIED BY FACTOR 0.3 TO LIMIT SLIP 
KOSTROV SLIP FUNCTION

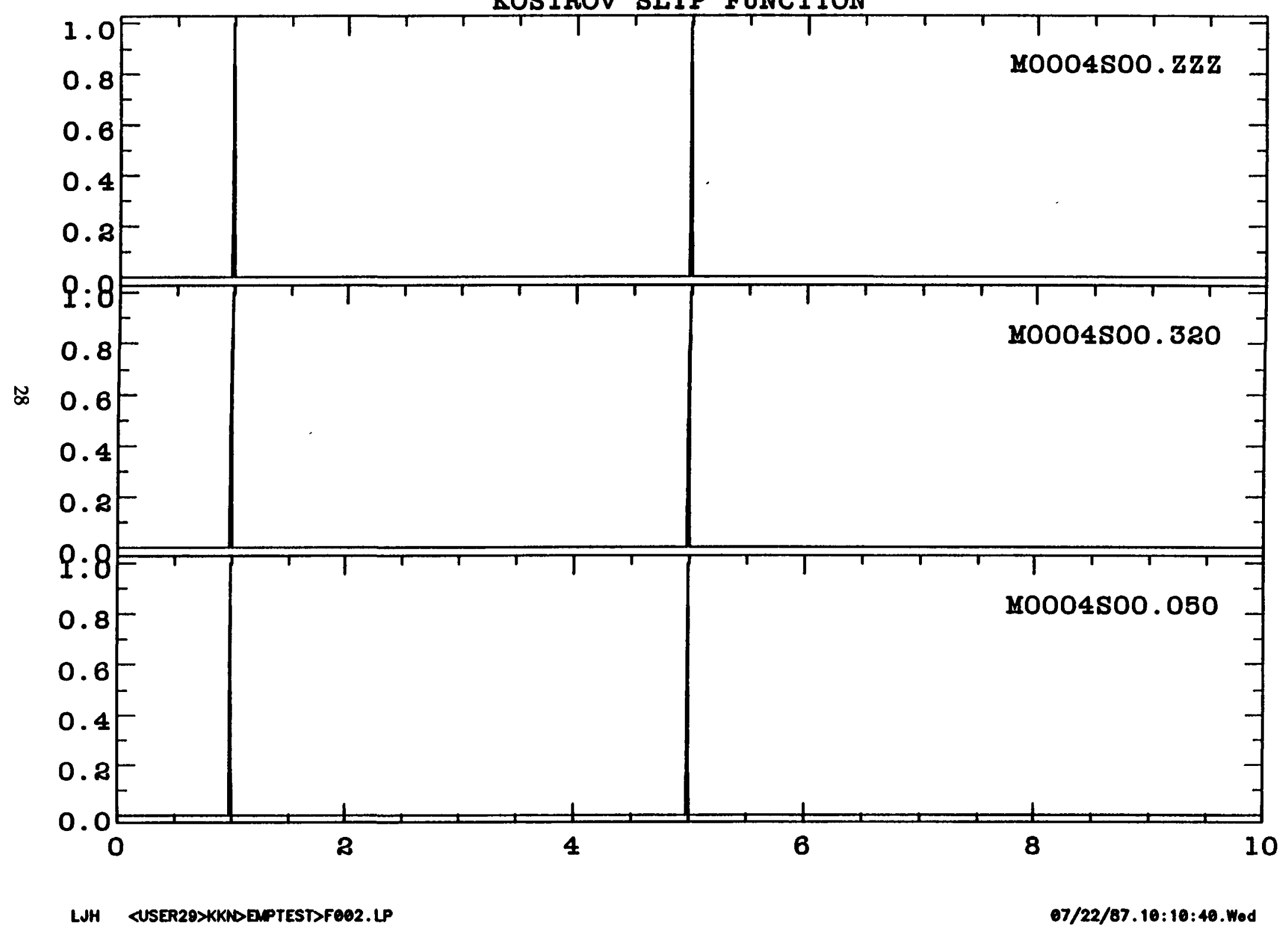

ficare? 
SYNTHESIS OF EVENT 0004 AT STATION

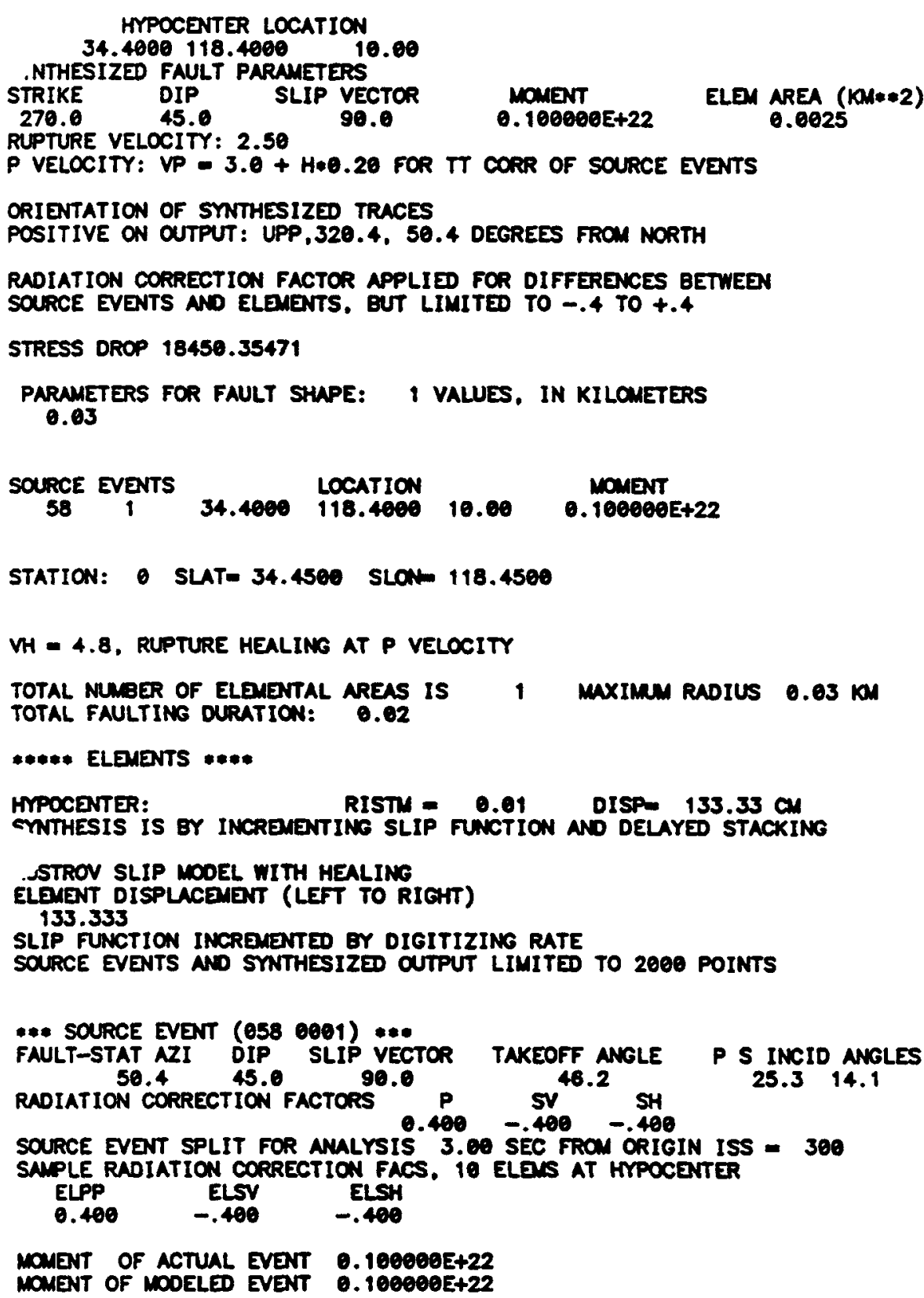


HASKEL RANP SLIP FUNCTION

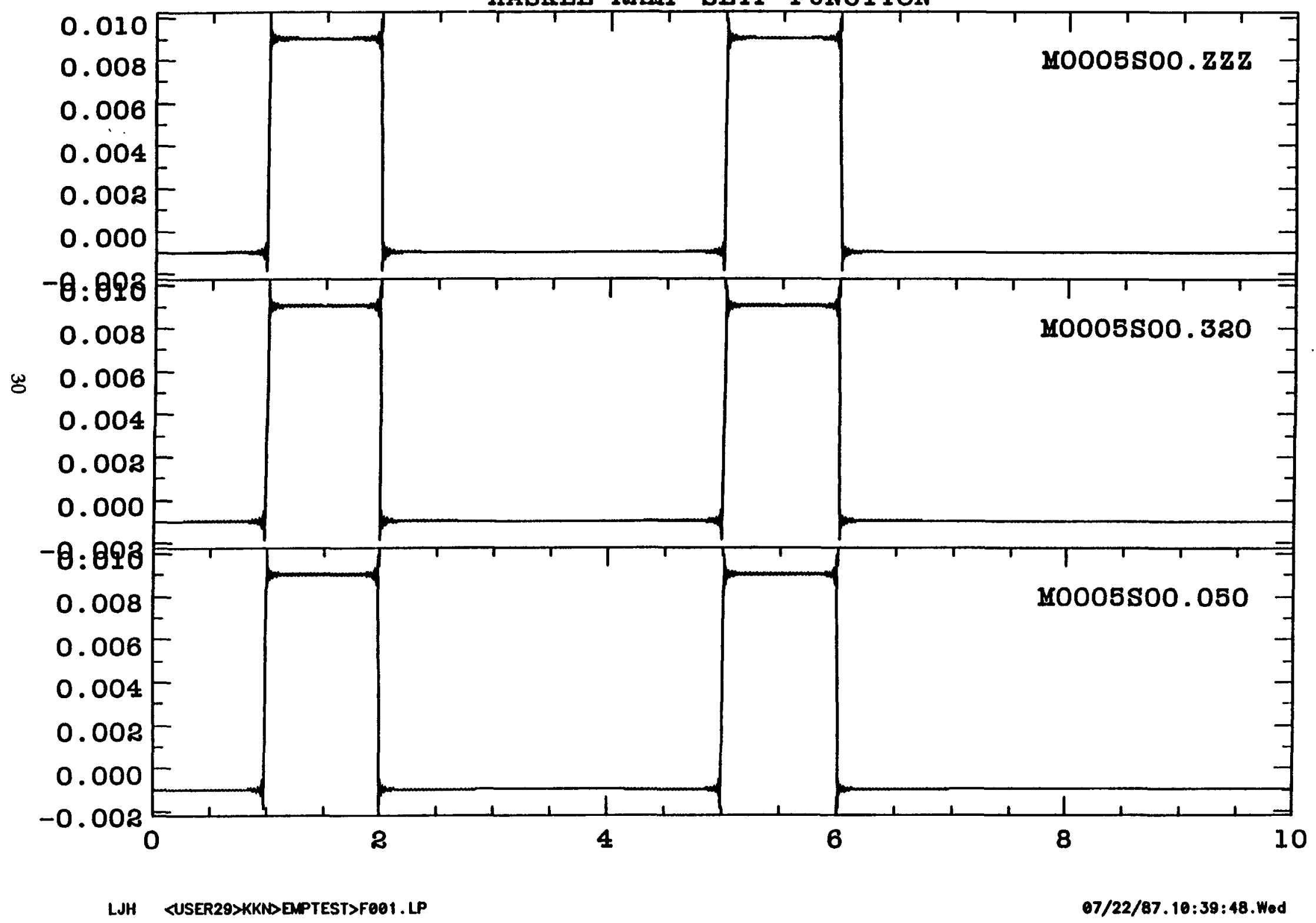

fiaure 8 
SYNTHESIS OF EVENT $000 S$ AT STATION

$$
\text { figure } 8
$$

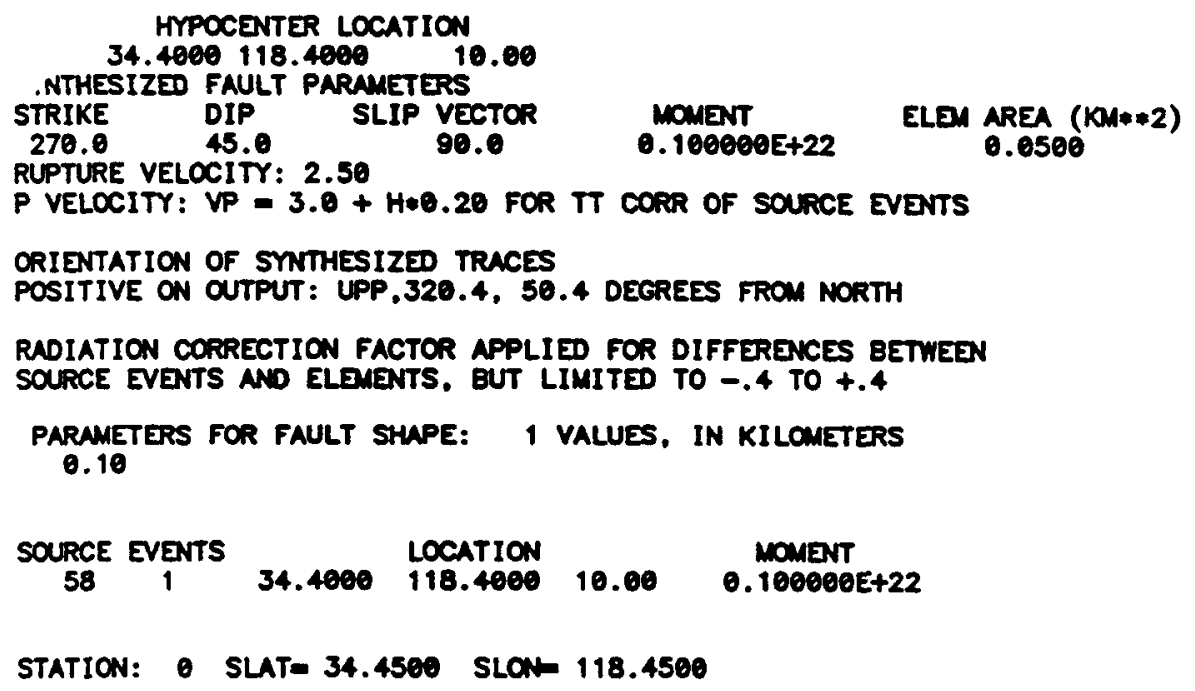

MONENT OF ACTUAL EVENT $0.100000 E+22$ MONENT OF MODELED EVENT $0.100000 E+22$ 


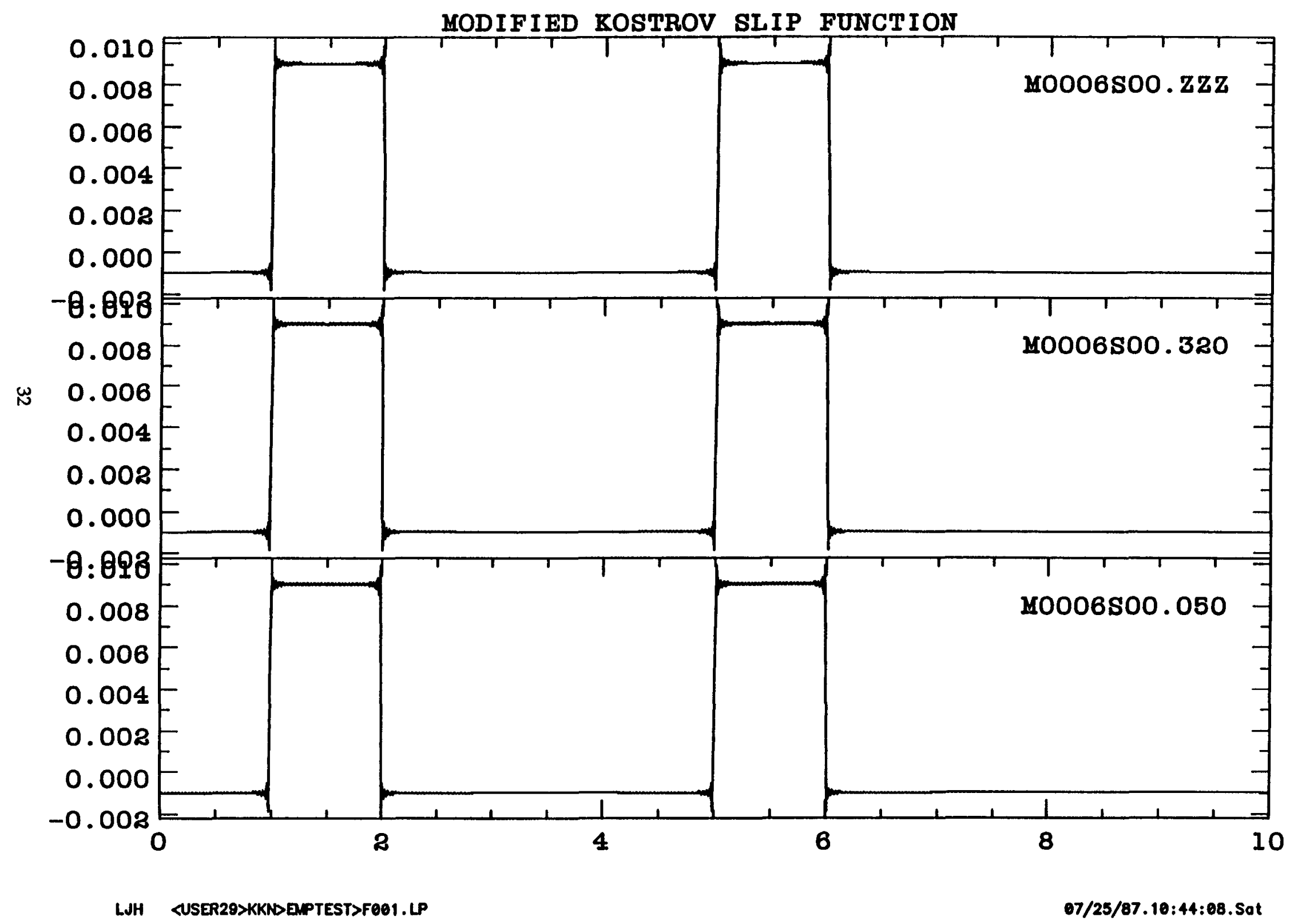

ficure 9 
SYNTHESIS OF EVENT BOEG AT STATION $\theta$

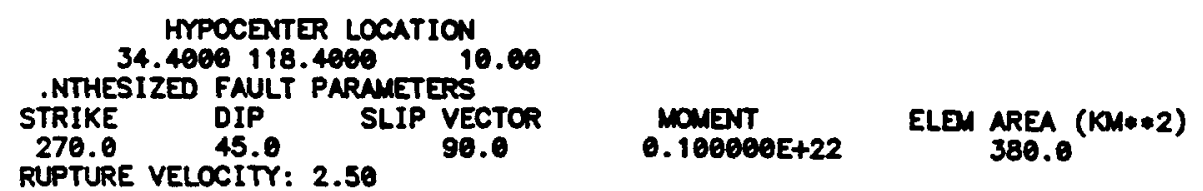

RUPTURE VELOCITY: 2.50

P VELOCITY: VP $=3.0+H * 0.20$ FOR TT CORR OF SOURCE EVENTS

ORIENTATION OF SMNTHESIZED TRACES

POSITIVE ON OUTPUT: UPP, 320.4, 50.4 DEGREES FROM NORTH

RADIATION CORRECTION FACTCR APPLIEO FOR DIFFERENCES BETWEEN SOURCE EVENTS AND ELEMENTS, BUT LIMITED TO -.4 TO +.4

STRESS DROP

0.60116915 PARAMETERS FOR FAULT SHMPE: I VALUES. IN KILOMETERS
11.00

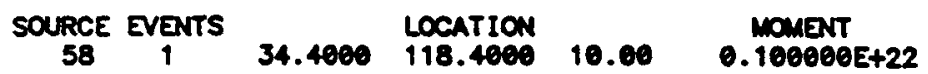

STATION: O SLAT= 34.4500 SLON 118.4500

VH $=4.8$, RUPTURE HEALING AT P VELOCITY

TOTAL NUBER OF ELEMINTAL AREAS IS 1 MXIMUM RADIUS $11.00 \mathrm{KM}$ TOTAL FAULTING DURATION: $\mathbf{5 . 4 0}$

\#*** EL̨EENTS \#.**

HIPOCENTER: RISTM = 1.00 DISP $0.00 \mathrm{CM}$

.NTHESIS IS BY CONOLUTION OF SOURCE EVENTS WITH SLIP FUNTION.

DECONVLVED WITH STEP FUNCTION. ENCH SOURCE EVENT IS SPLIT BETWEEN P AND S.

AND CONPUTATION IS DONE IN FRECUENCY DOMIN. FFT FOR EACH SOURCE EVENT ONCE.

RANP DISPLACENENT FUNCTION - MOOIFIED KOSTRON

SLIP AMPLITUDE ND RISE TIME CONSTRAINED BY SELF-SIMILAR KOSTRON SLIP. MULTIPLIED BY FACTOR 0.3 TO LIMIT SLIP

ELEMENT DISPLACEMENT (LEFT TO RIGHT)

FIRST 1 ELEMENTS

0.001

SPECTRAL DIMENSION IS 2048 POINTS. IF TRACES ARE GREATER THAN 15 SECONDS YOU GET WRAP-AROUND IN MAIN SEISIDGRAM

*** SOURCE EVENT (058 0601) ***

FAULT-STAT AZI DIP SLIP VECTOR TAKEOFF ANGLE P S INCID ANGLES

RADIATION CORRECTION FACTORS $P$ PV

SOURCE EVENT SPLIT FOR AMLYSIS 3.00 SEC FROM ORIGIN ISS = 300

SAMLE RADIATION COFRECTION FACS, 10 ELENS AT HYPOCENTER

$$
\begin{array}{lrr}
\text { ELPP } & \text { ELSV } & \text { ELSH } \\
0.400 & -.400 & -.400
\end{array}
$$

MOMENT OF ACTUAL EVENT $0.1000005+22$

MONENT OF MOOELED EVENT $0.160000 E+22$ 
KOSTROV SLIP FUNCTION

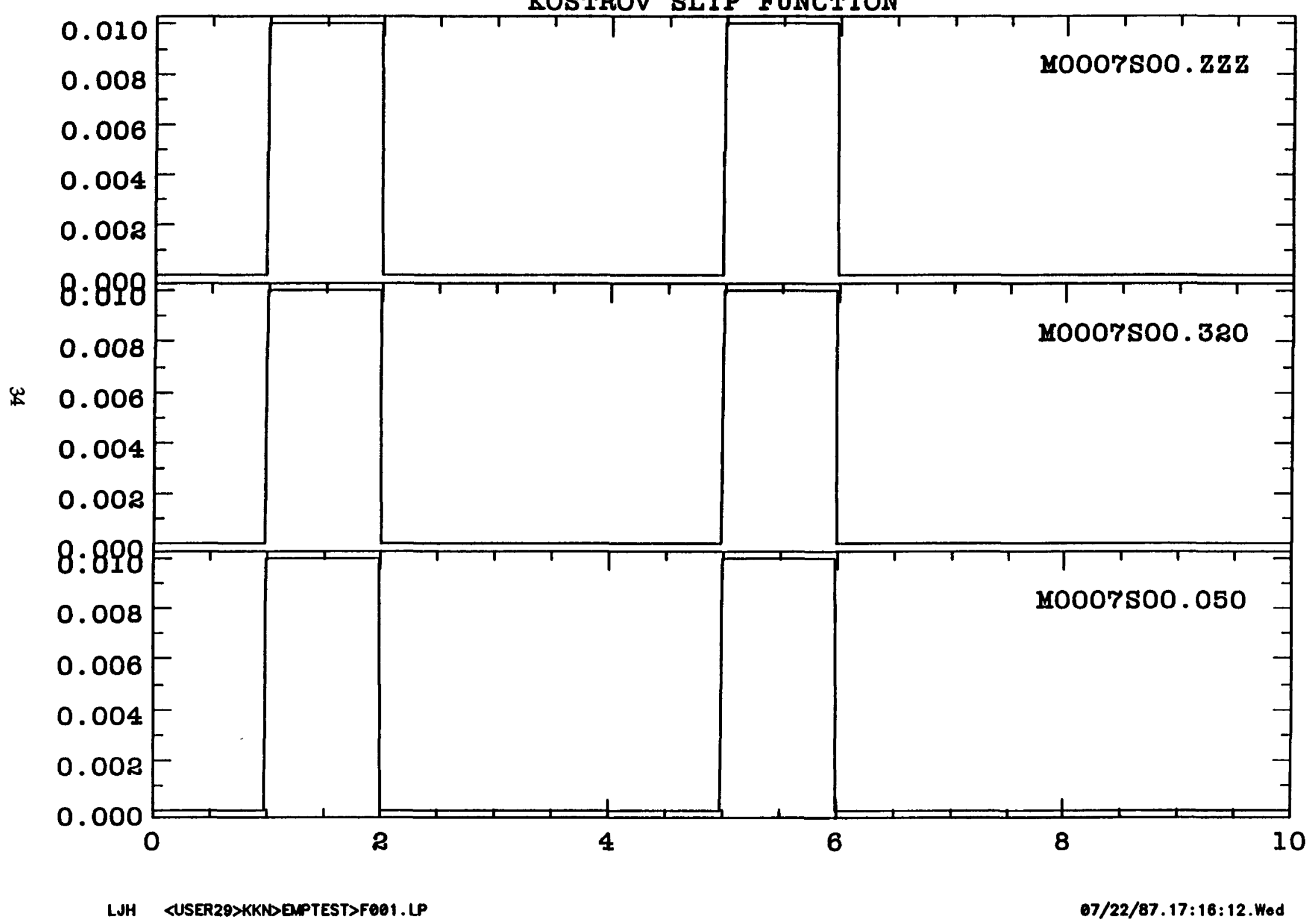

figure 10 
SYNTHESIS OF EVENT DOE7 AT STATION

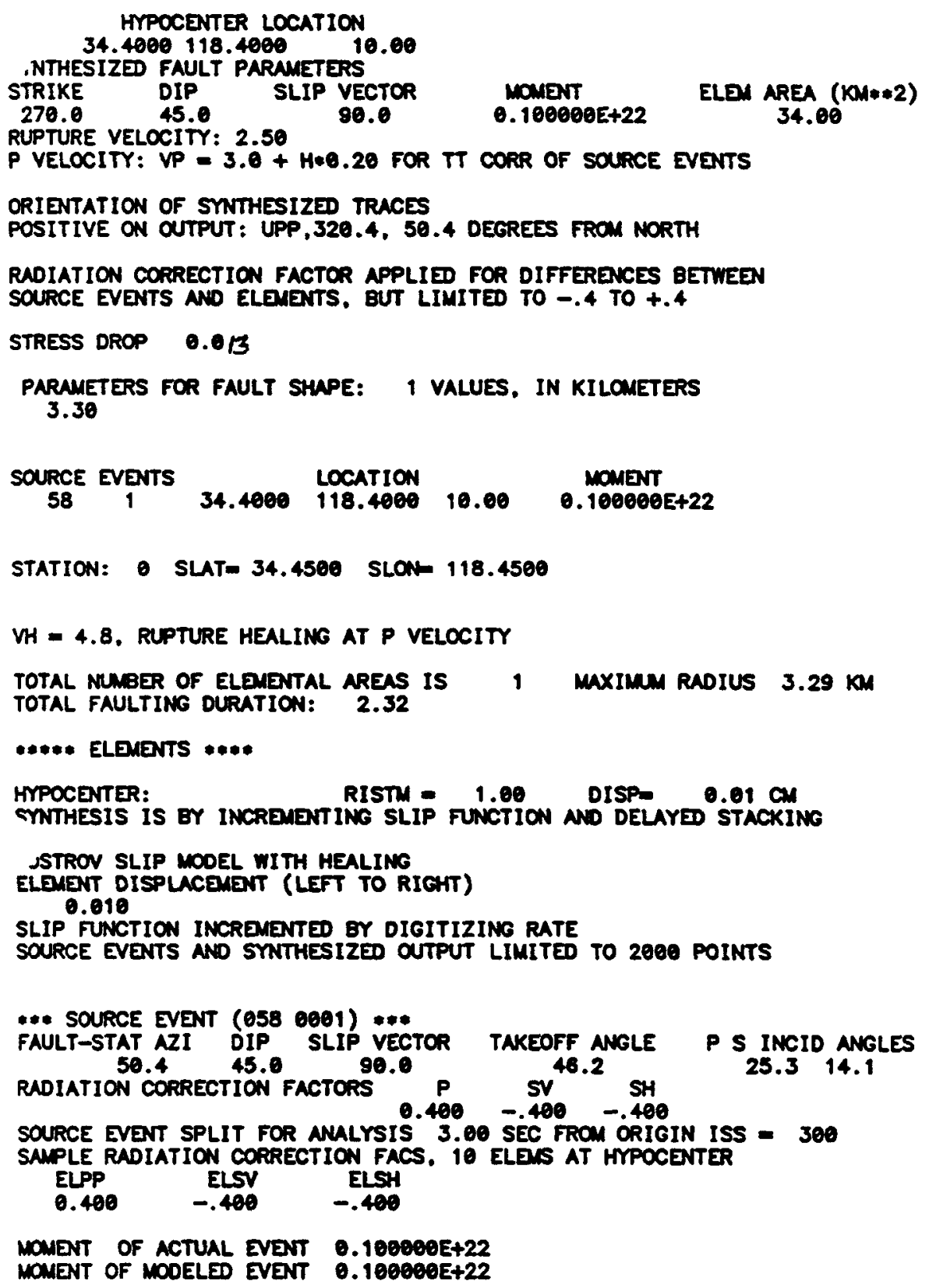




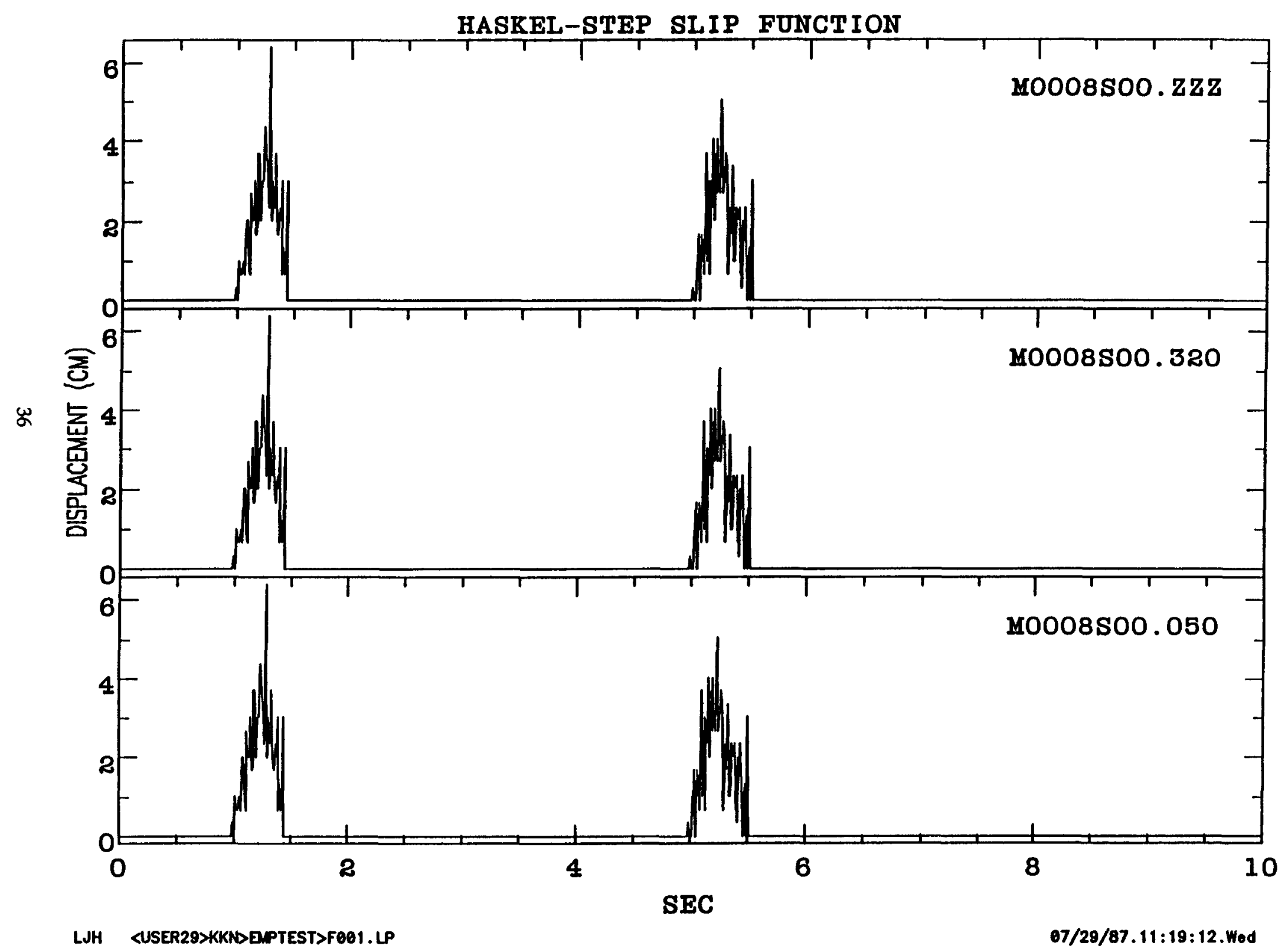

ficure II 
SYNTHESIS OF EVENT 0008 AT STATION 0

MYPOCENTER LOCATION

$34.4000118 .4000 \quad 10.00$

- NTHESIZED FAULT PARAMETERS

STTRIKE DIP SLIP VECTOR

$270.0 \quad 45.0 \quad 90.0$

RUPTURE VELOCITY: 2.50

P VELOCITY: VP $=3.0+H * 0.20$ FOR TT CORR OF SOURCE EVENTS

ORIENTATION OF SYNTHESIZED TRACES

POSITIVE ON OUTPUT: UPP, 320.4, 50.4 DECREES FROM NORTH

RAOIATION CORRECTION FACTOR APPLIED FOR OIFFERENCES BETWEEN

SOURCE EVENTS AND ELENENTS, BUT LIMITED TO -.4 TO +.4

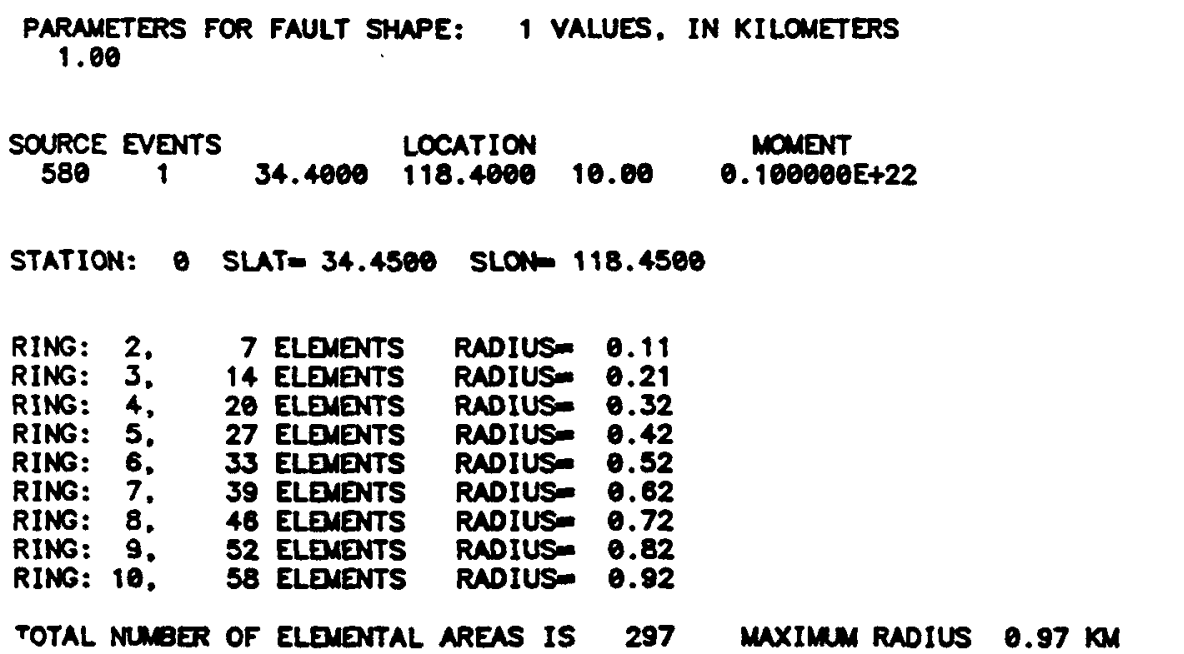

TWO DIMENSIOML HASKEL RUPTURE MODEL WITH STEP SLIP FUNCTION

EACH ELEMENT HAS THE SAME DISPLACEMENT: $11.2 \mathrm{CM}$

TOTAL FAULTING DURATION 0.37 SECONDS

SLIP FUNCTION INCREMDNTED BY DIGITIZING RATE

SOURCE EVENTS AND SWTHESIZED OUTPUT LIMITED TO 1000 POINTS

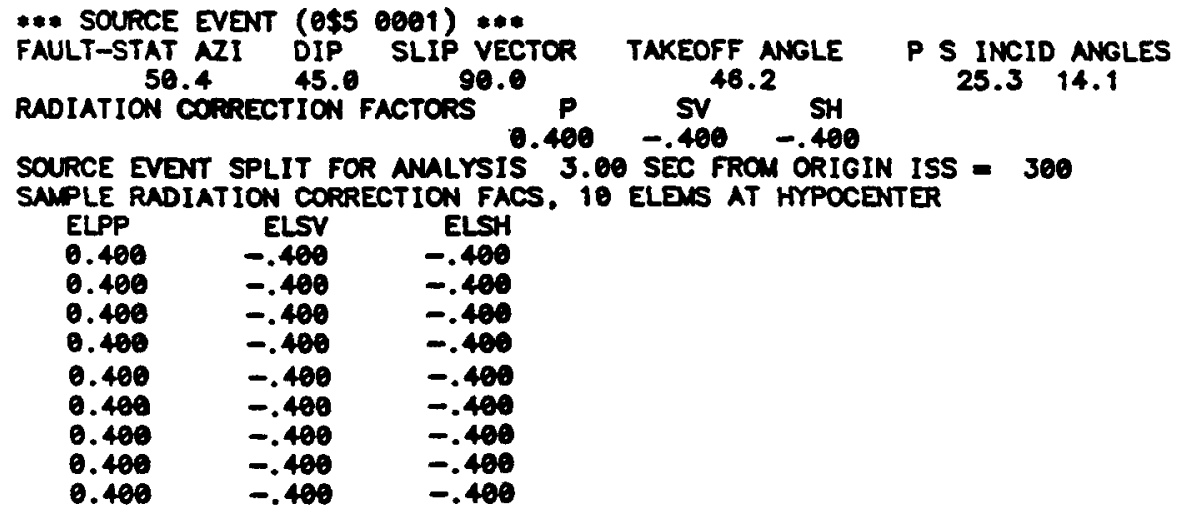

MONENT OF ACTUAL EVENT $0.100000 E+24$ MONENT OF IDDELED EVENT $0.990990 E+23$ 


\section{HASKEL-RAMP SLIP FUNCTION}

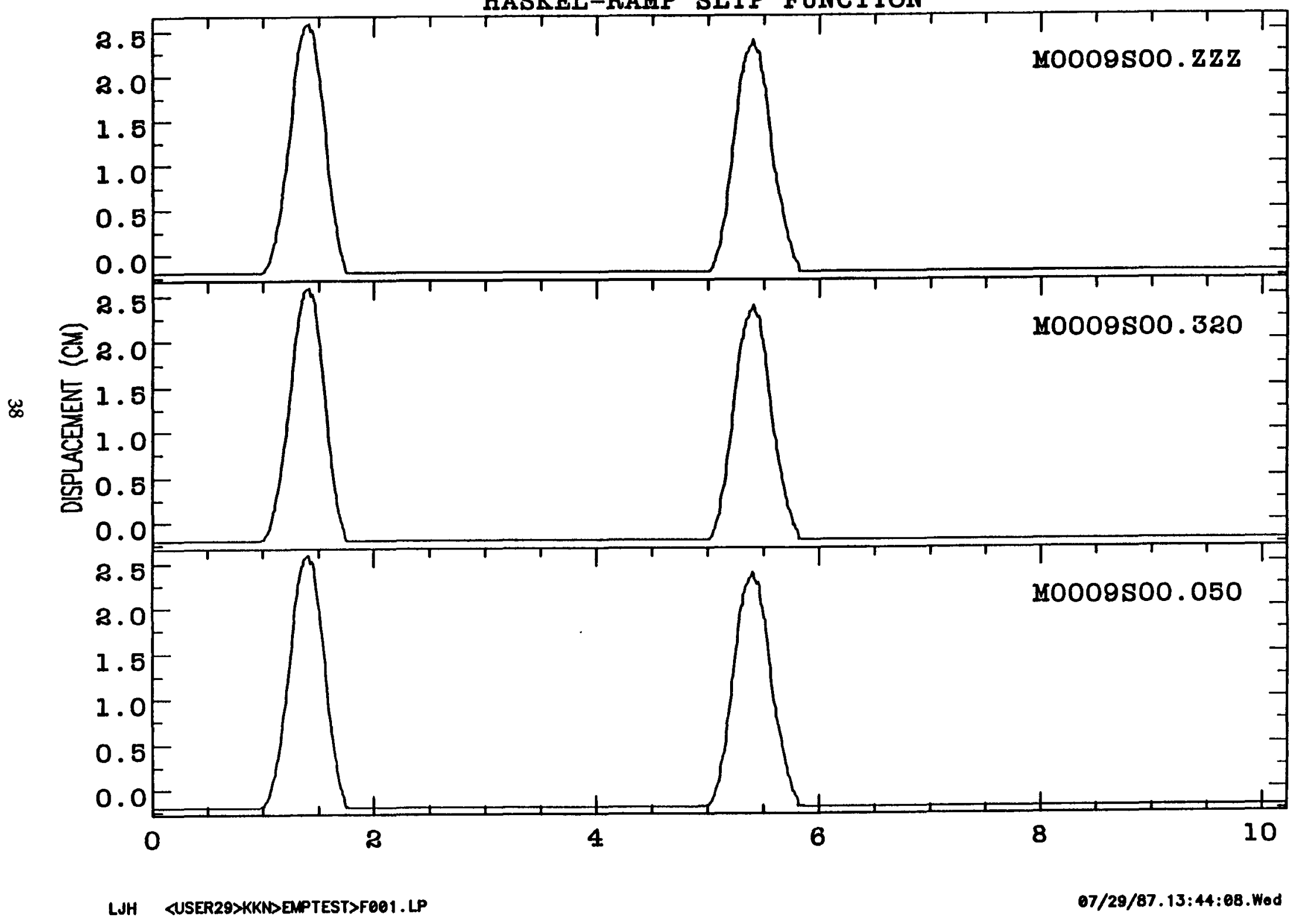

fisure 12 
SYNTHESIS OF EVENT 0009 AT STATION 0

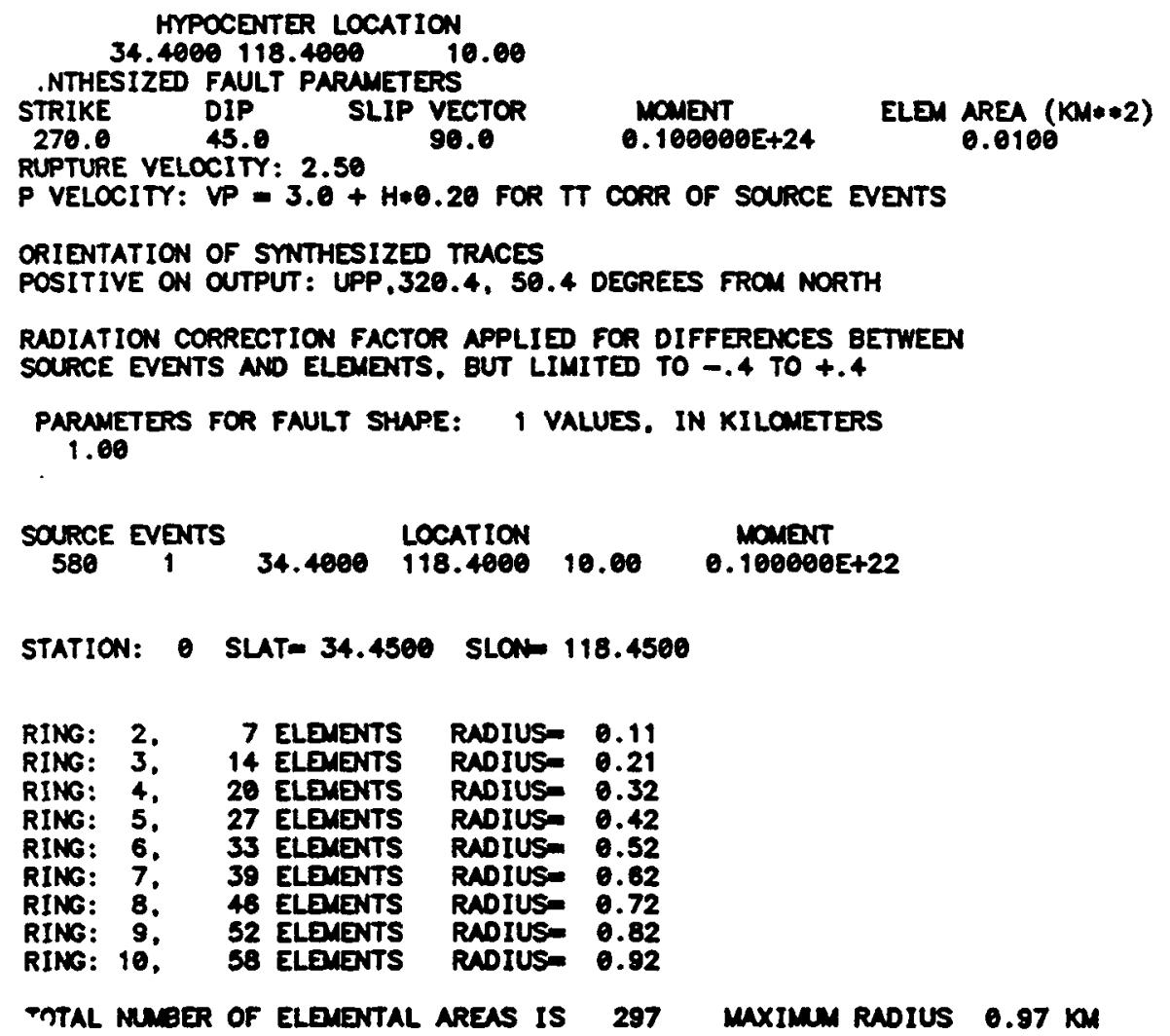

STNTHESIS IS EY CONOLUTION OF SOURCE EVENTS WITH SLIP FUNCTION,

DECONVOLVED WITH STEP FUNCTION. EACH SOURCE EVENT IS SPLIT BETWEEN P AND S,

ANO CONPUTATION IS DONE IN FRECUENCY DOMAIN. FFT FOR EACH SOURCE EVENT ONCE.

TWO DIMENSIONAL HASKEL RUPTURE MODEL WITH RAMP SLIP FUNCTION

EACH ELEMENT HAS SAME OISPLACEMENT: $11.2 \mathrm{CM}$

RISE TIME $=0.30$ TOTAL FAULTING DURATION 0.67

SPECTRAL DIMENSION IS 1024 POINTS. IF TRACES ARE GREATER THAN 5 SECONDS YOU GET WRAP-AROAND IN MAIN SEISIDORAM

*** SOURCE EVENT (055 6001) ***

FAULT-STAT AZI DIP SLIP VECTOR TAKEOFF ANGLE P 5 INCID ANGLES 50.4
RADIATION CORRECTION FACTORS

SOURCE EVENT SPLIT FOR AMLYSIS 3.00 SEC FROM ORIGIN ISS - 300

SNMLE RNDIATION CORRECTION FACS, 10 ELENS AT MYPOCENTER

$\begin{array}{lll}\text { ELPP } & \text { ELSV } & \text { ELSH } \\ 0.400 & -.400 & -.400 \\ 0.400 & -.400 & -.400 \\ 0.400 & -.400 & -.400 \\ 0.400 & -.400 & -.400 \\ 0.400 & -.400 & -.400 \\ 0.400 & -.400 & -.400 \\ 0.400 & -.400 & -.400 \\ 0.400 & -.400 & -.400 \\ 0.400 & -.400 & -.400\end{array}$

MOUENT OF ACTUAL EVINT $0.100000 E+24$ MOUENT OF MODELED EVENT $0.999990 E+23$ 
MODIFIED KOSTROV SLIP FUNCTION

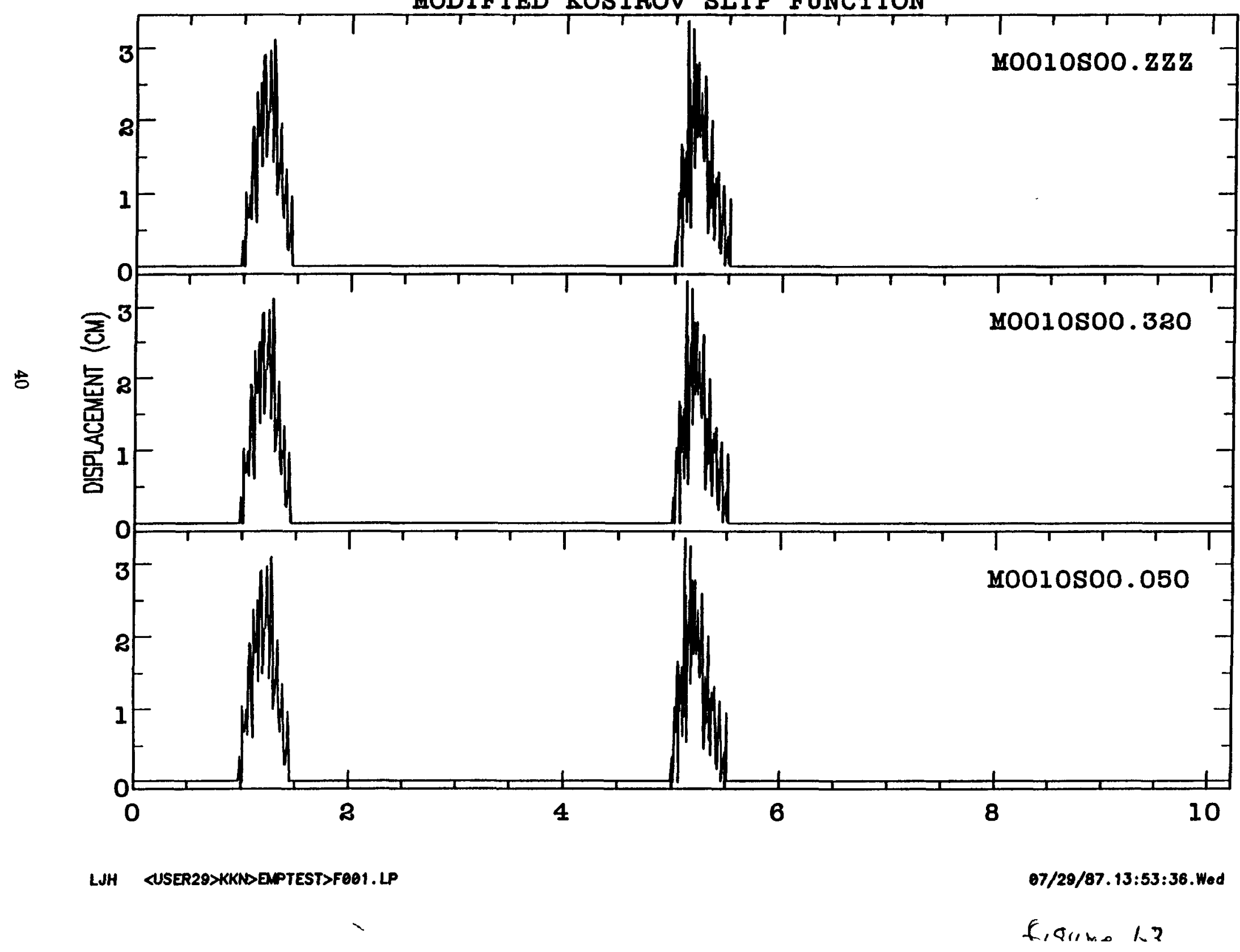


SHNTHESIS OF EVENT BOIO AT STATION

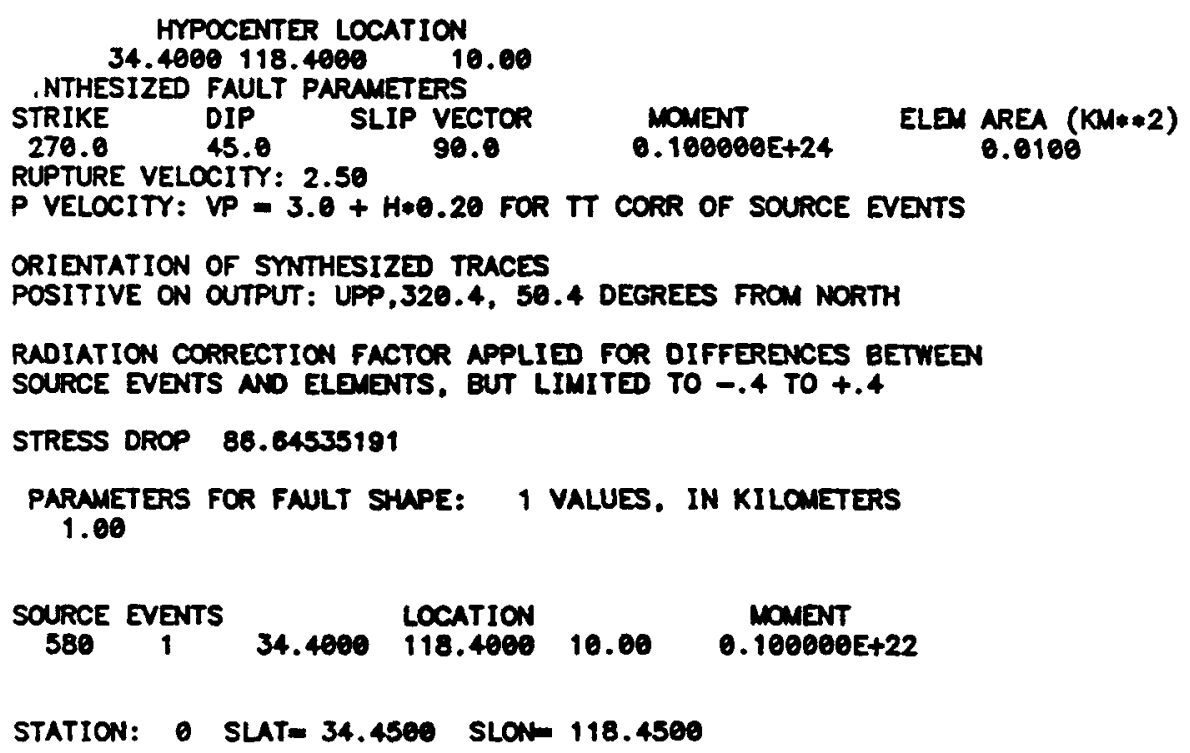

VH $=$ 4.8, RUPTURE HEALING AT P VELOCITY

RING: 2.7 ELEMENTS RADIUS- 0.11

RING: 3, 14 ELEMENTS RADIUS- 0.21

RING: 4, 20 ELEMENTS RADIUS- 0.32

RING: 5, 27 ELEMENTS RADIUSO 0.42

RING: $6, \quad 33$ ELONENTS RADIUS- 0.52

nING: 7. 39 ELENENTS RADIUSO 0.82

VG: 8,46 ELDMENTS RADIUS- 0.72

.NG: 9, 52 ELEMENTS RADIUSO 0.82

RING: 10; 58 ELDIENTS RADIUS- 0.92

TOTAL NUBER OF ELENENTAL AREAS IS 297 MAXIMNA RADIUS $0.97 \mathrm{kM}$

TOTAL FAULTING DURATION: 0.40

MAIMU RADIUS $0.97 \mathrm{KM}$

***** ELEMENTS ****

HMPOCENTER: $\quad$ RISTM $=0.18$ DISP $11.48 \mathrm{cM}$

SYNTHESIS IS BY CONVOLUTION OF SOURCE EVENTS WITH SLIP FUNCTION,

DECONVOLVED WITH STEP FUNCTION. EACH SOURCE EVENT IS SPLIT BETWEEN P AND S.

AND COMPUTATION IS DONE IN FREOUENCY DOMAIN. FFT FOR EACH SOURCE EVENT ONCE.

RANP DISPLACEMENT FUNCTION - MODIFIED KOSTROV

SLIP AMPLITUDE AND RISE TIME CONSTRAINED EY SELF-SIMILAR KOSTROV SLIP.

MLTIPLIED BY FACTOR 0.3 TO LIMIT SLIP

ELEMENT DISPLACEMENT (LEFT TO RIGHT)

FIRST 160 ELBMENTS

$\begin{array}{llllllll}11.484 & 11.352 & 11.352 & 11.352 & 11.352 & 11.352 & 11.352 & 11.352\end{array}$ 


\begin{tabular}{|c|c|c|c|c|c|c|c|}
\hline $\begin{array}{r}10.839 \\
10.839 \\
10.261 \\
10.261 \\
10.261 \\
9.605 \\
9.604 \\
9.604 \\
8.848 \\
8.848 \\
8.848 \\
8.847 \\
7.975 \\
7.975 \\
7.975 \\
7.975 \\
7.975 \\
6.927 \\
6.927 \\
\text { CTRAL }\end{array}$ & $\begin{array}{r}10.839 \\
10.839 \\
10.261 \\
10.261 \\
10.261 \\
9.665 \\
9.605 \\
9.604 \\
8.847 \\
8.848 \\
8.848 \\
8.848 \\
7.975 \\
7.975 \\
7.975 \\
7.975 \\
7.975 \\
6.927 \\
6.927\end{array}$ & $\begin{array}{r}10.839 \\
10.839 \\
10.261 \\
10.261 \\
9.605 \\
9.605 \\
9.605 \\
9.605 \\
8.847 \\
8.848 \\
8.848 \\
8.848 \\
7.975 \\
7.876 \\
7.975 \\
7.975 \\
7.975 \\
6.927 \\
8.927 \\
1024\end{array}$ & $\begin{array}{r}10.839 \\
10.839 \\
10.261 \\
10.261 \\
9.605 \\
9.605 \\
9.605 \\
9.605 \\
8.848 \\
8.848 \\
8.848 \\
8.848 \\
7.975 \\
7.975 \\
7.975 \\
7.875 \\
7.975 \\
8.927 \\
6.927 \\
\text { INTS. IF }\end{array}$ & $\begin{array}{r}10.839 \\
10.839 \\
10.261 \\
10.281 \\
9.684 \\
9.605 \\
9.605 \\
9.605 \\
8.848 \\
8.847 \\
8.848 \\
8.848 \\
7.975 \\
7.875 \\
7.875 \\
7.975 \\
7.976 \\
6.927 \\
6.027 \\
\text { TRACES }\end{array}$ & $\begin{array}{c}10.839 \\
10.840 \\
10.261 \\
10.261 \\
9.604 \\
9.804 \\
9.605 \\
8.848 \\
8.848 \\
8.847 \\
8.848 \\
8.848 \\
7.975 \\
7.975 \\
7.975 \\
7.975 \\
6.927 \\
6.927 \\
6.927 \\
\text { E GREATER }\end{array}$ & $\begin{array}{r}10.840 \\
10.261 \\
10.261 \\
10.261 \\
9.604 \\
9.604 \\
9.604 \\
8.848 \\
8.848 \\
8.848 \\
8.848 \\
7.975 \\
7.975 \\
7.975 \\
7.975 \\
7.973 \\
6.927 \\
6.927 \\
6.927 \\
\text { THWN }\end{array}$ & $\begin{array}{r}10.839 \\
10.261 \\
10.261 \\
10.261 \\
9.604 \\
9.604 \\
9.604 \\
8.848 \\
8.848 \\
8.848 \\
8.847 \\
7.975 \\
7.975 \\
7.975 \\
7.976 \\
7.975 \\
6.927 \\
6.927 \\
6.927 \\
\text { SECONDS }\end{array}$ \\
\hline
\end{tabular}

** SOURCE EVETT (0\$5 6001) \#.*
FAULT-STAT AZ
DIP SLIP VECTOR
TAKEOFF AMGLE 50.4 45.0
P S INCID ANGLES 25.314 .1 90.0 $\mathrm{SV}^{46.2} \mathrm{SH}$ RADIATION CORRECTION FACTORS $0.400-.400-.400$

figure $13 \mathrm{~cm}$.

SOURCE EVENT SPLIT FOR AMLYSIS 3.00 SEC FROM ORIGIN ISS = 300 SAPLE RADIATION CORRECTION FACS, io ELENS AT HMPOCENTER

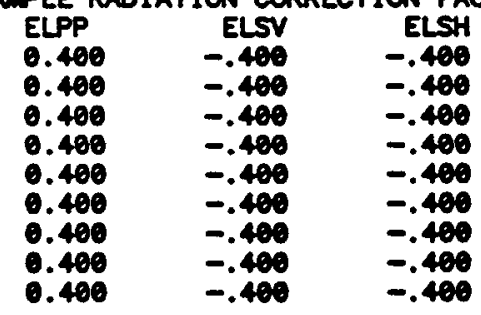

MOMENT OF ACTUAL EVENT $0.100000 E+24$ MOMENT OF MODELED EVENT $0.100000 E+24$ 


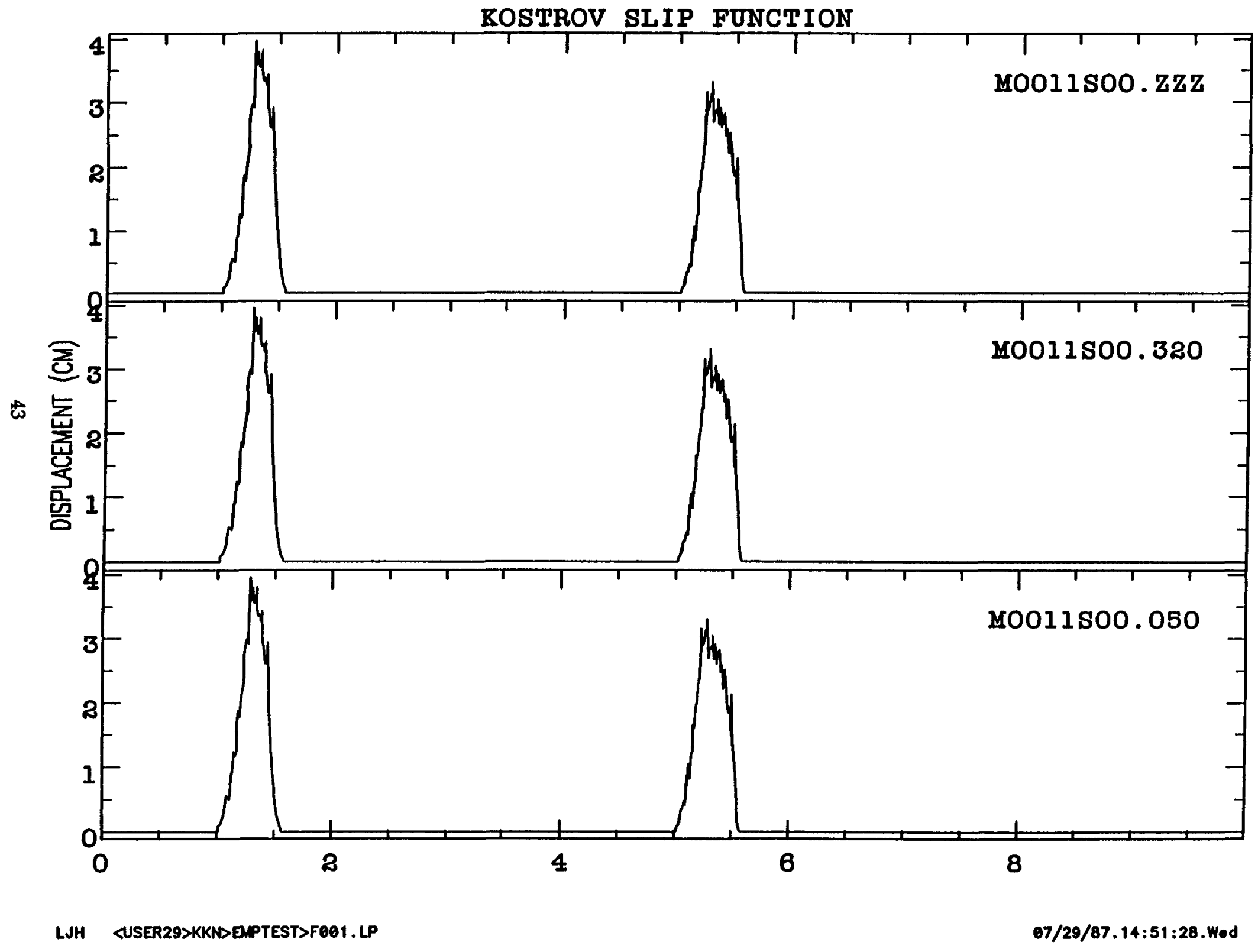

fiaure 14 
SWNTHESIS OF EVENT 0011 AT STATION O

HYPOCENTER LOCATION

$34.4000118 .4000 \quad 10.00$

.NTHESIZED FAULT PARAMETERS

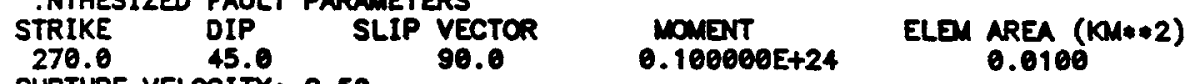

RUPTURE VELOCITY: 2.50

P VELOCITY: VP $-3.0+$ H*0.20 FOR TT CORR OF SOURCE EVENTS

ORIENTATION OF SMNTHESIZED TRACES

POSITIVE ON OUTPUT: UPP,320.4, 50.4 DEGREES FROA NORTH

RADIATION CORRECTION FACTOR APPLIED FOR DIFFERENCES BETWEEN

SOURCE EVENTS AND ELEMENTS. BUT LIMITED TO -.4 TO +.4

STRESS DROP 40.53242596

PARAMETERS FOR FAULT SHAPE: I VALUES. IN KILOMETERS

1.00

SOURCE EVENTS

580

34.4000 LOCATION

LOCATION MOMENT

STATION: SLAT= 34.4500 SLON= 118.4500

figure/y

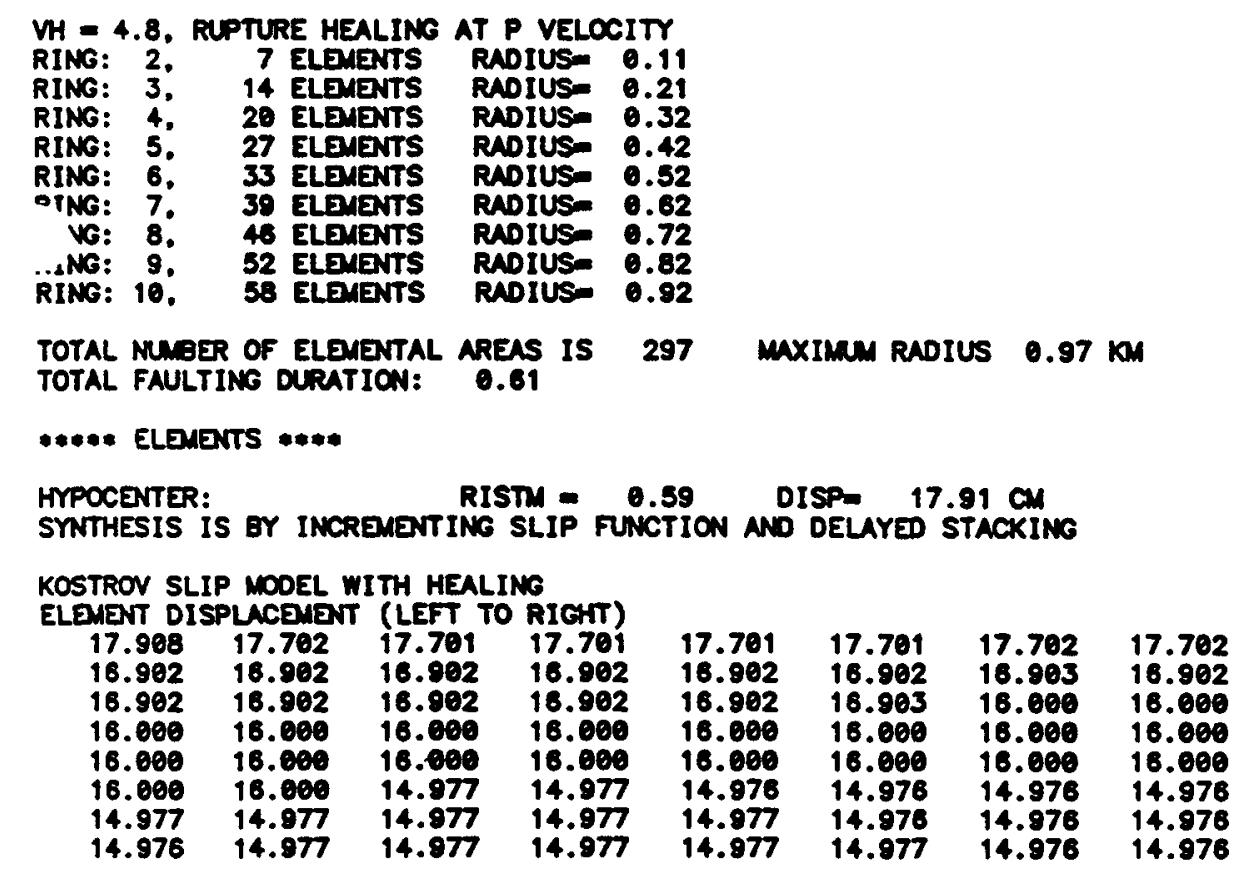




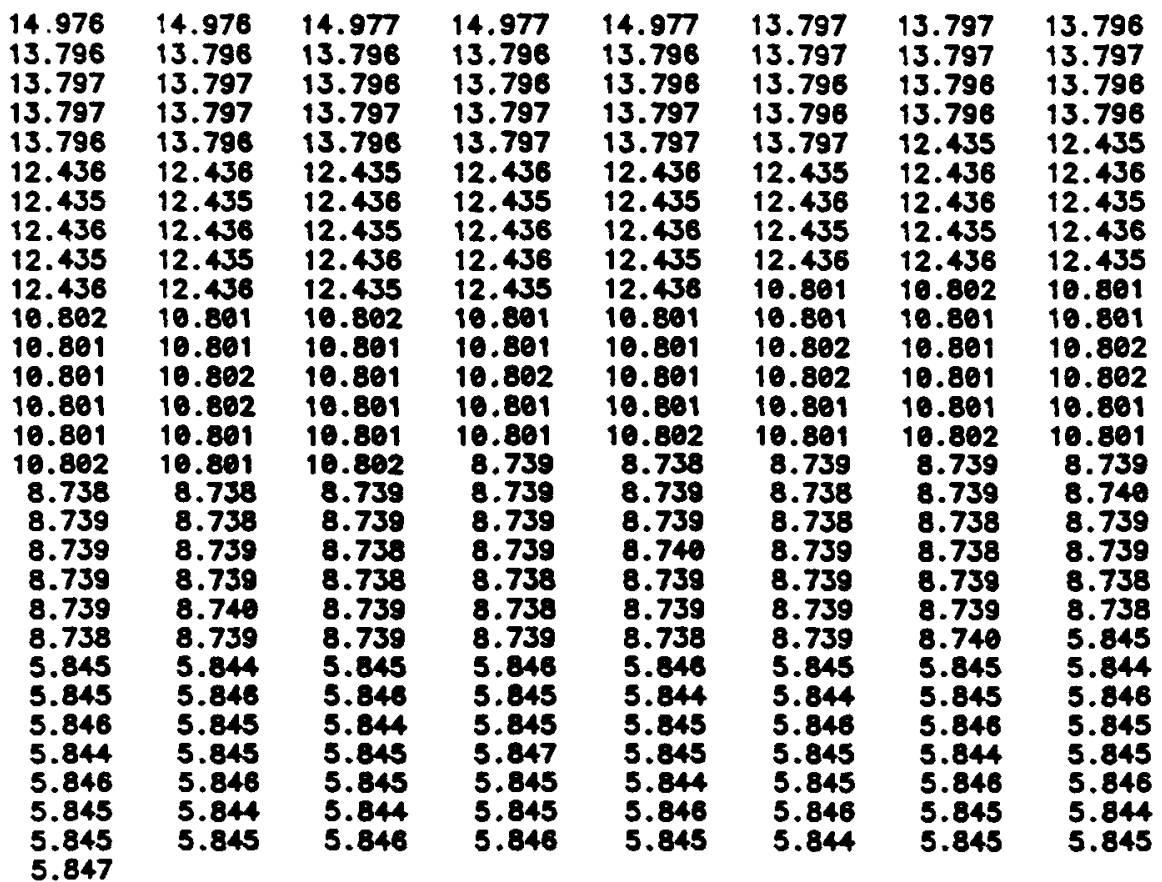

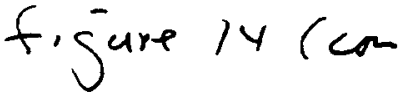

SLIP FUNCTION INCREMETED BY DIGITIZING RATE

SOURCE EVENTS ND SNNTHESIZED OUTPUT LIMITED TO 1000 POINTS

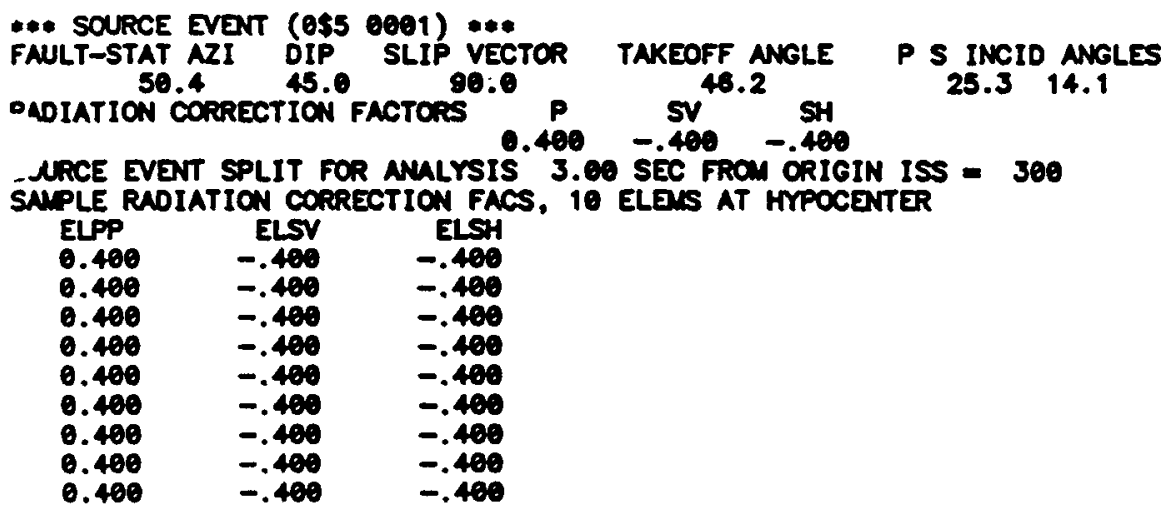

MOMENT OF ACTUAL EVENT $0.106000 E+24$ MOMENT OF MODELED EVINT $0.108000 E+24$ 


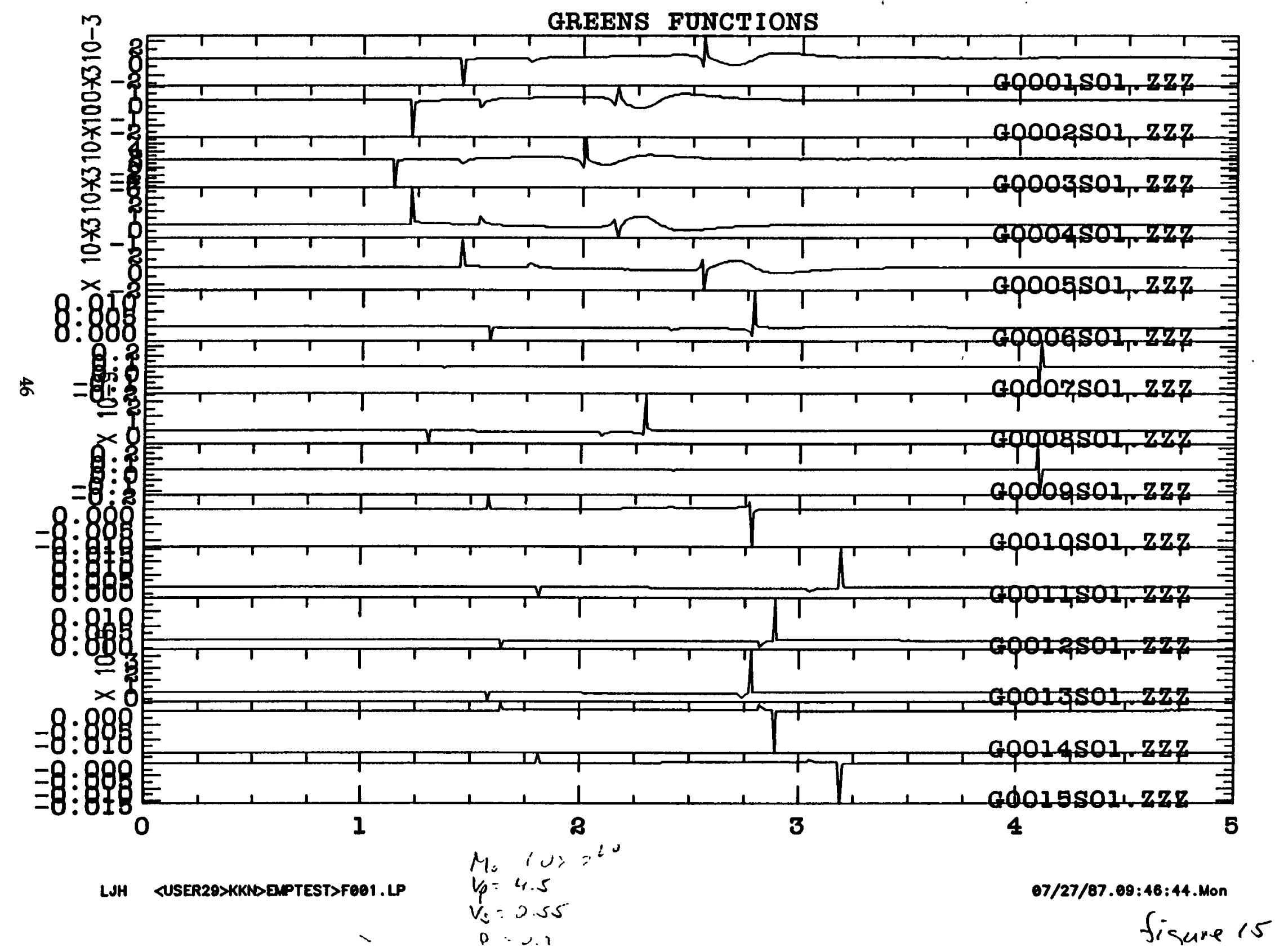




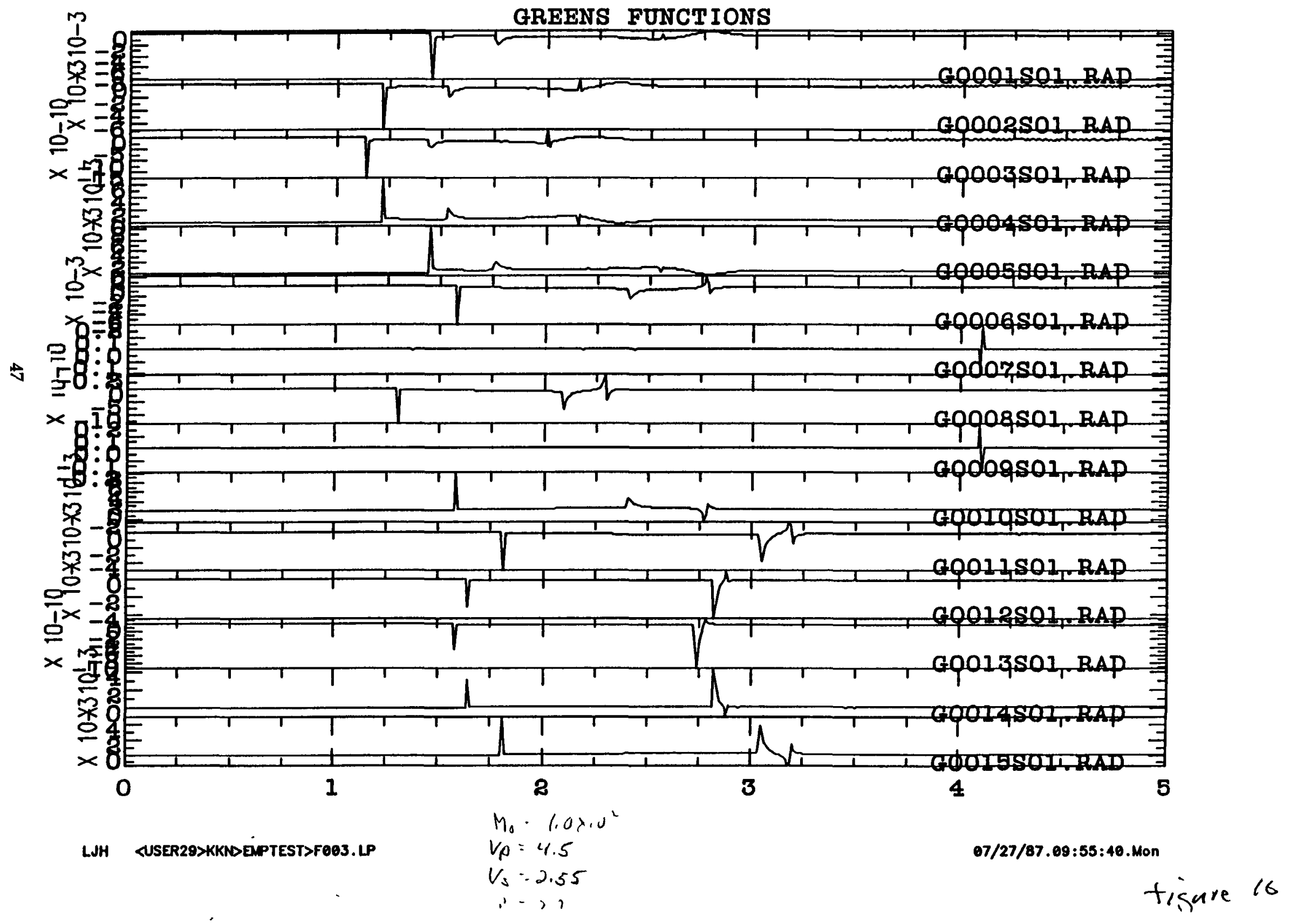


GREENS FUNCTIONS

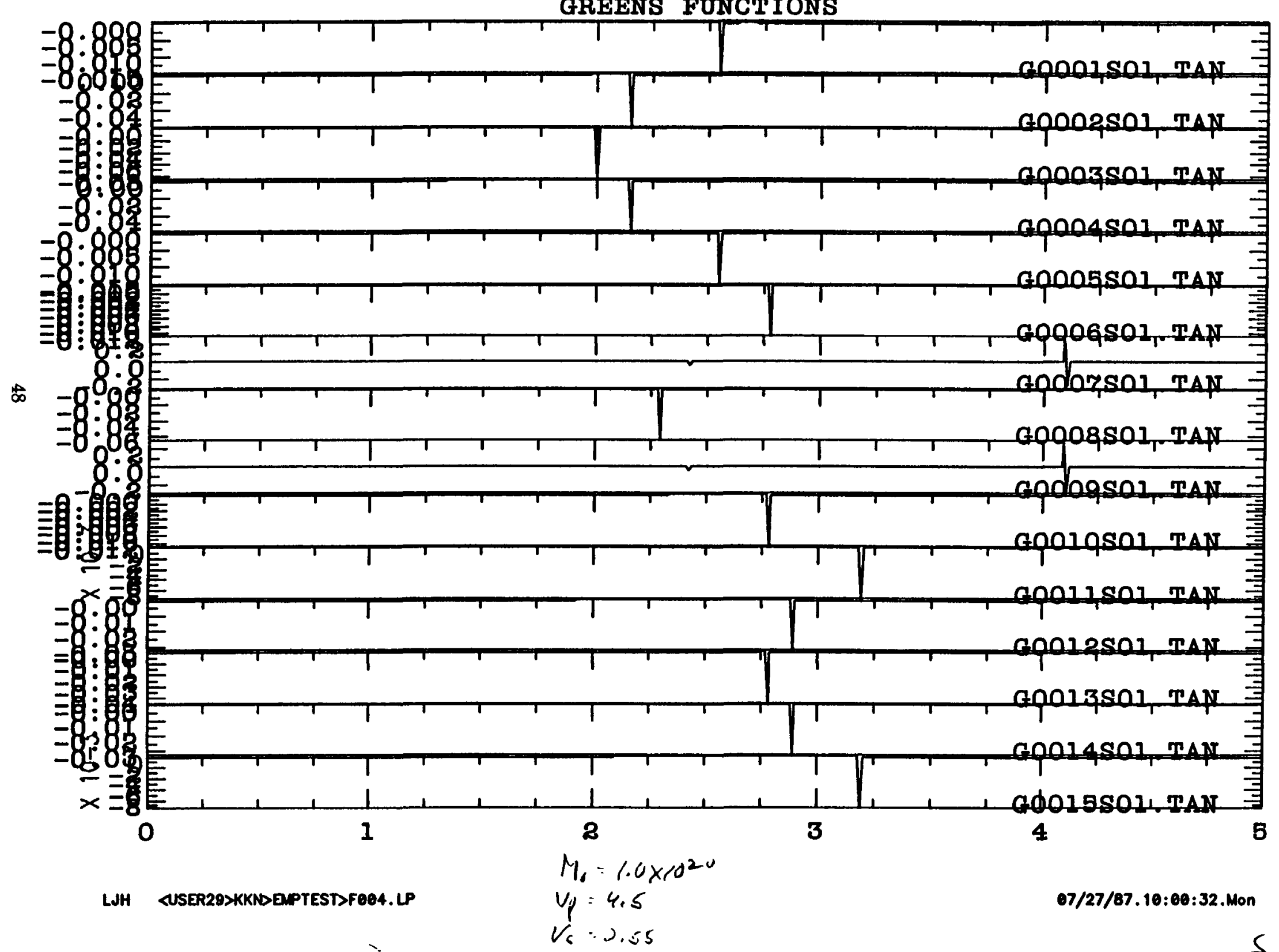

Sisure 17 


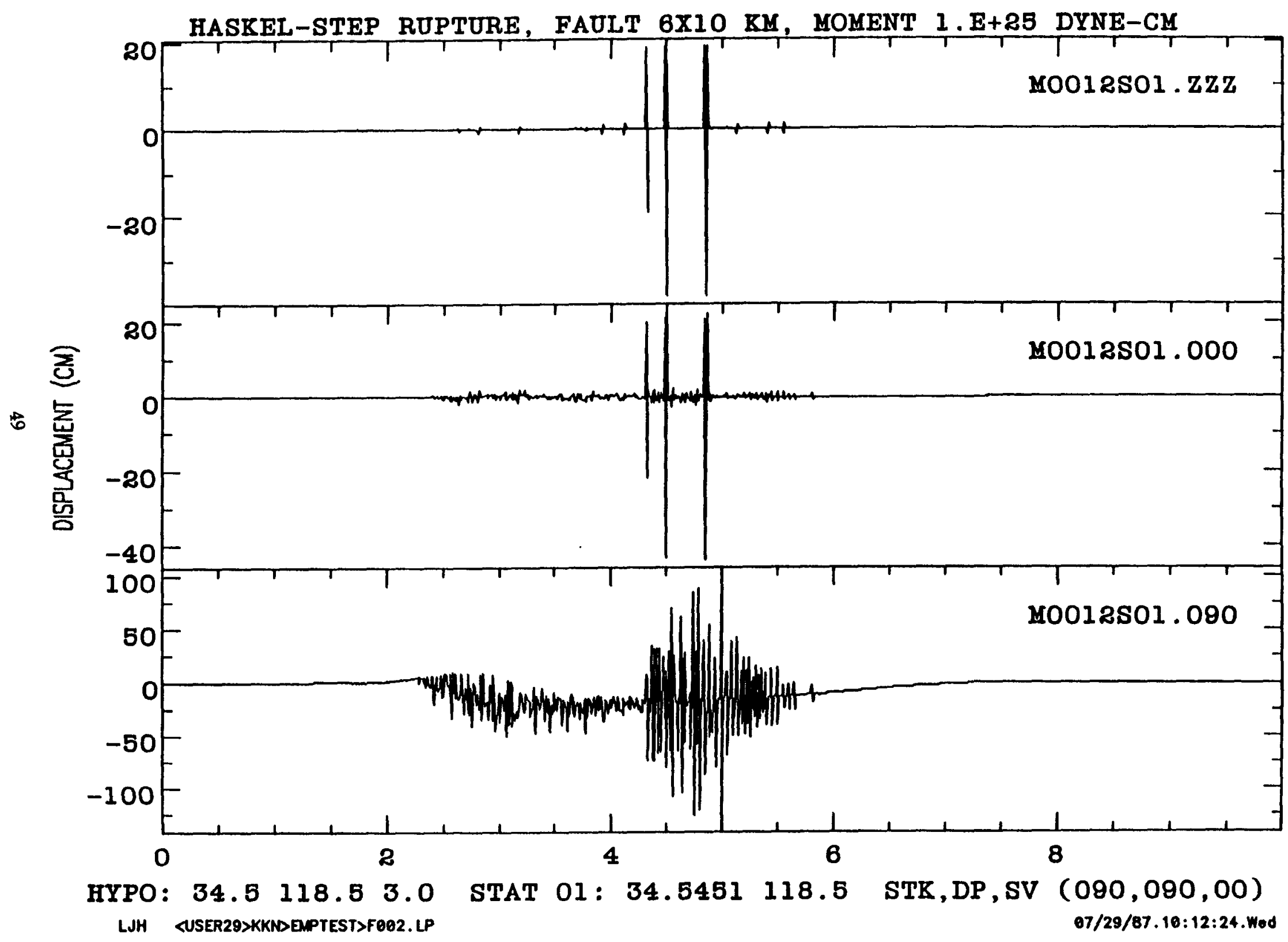

r. 
SMTHESIS OF EVENT 0012 AT STATION 1 HYPOCENTER LOCATION

$$
34.5000 \text { 118.5000 } 3 .
$$

VTHESIZED FAULT PARAMETERS

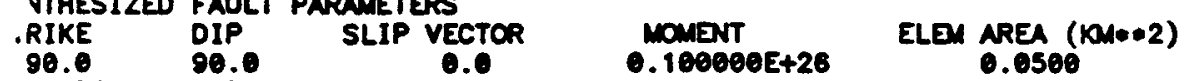

RUPTURE VELOCITY: 2.50

P VELOCITY: VP $-3.0+$ H*0.20 FOR TT CORR OF SOURCE EVENTS

ORIDNTATION OF SMTHESIZED TRACES

POSITIVE ON OUTPUT: UPP, 0.0, 90.0 DEGREES FROM NORTH

RADIATION CORRECTION FACTOR APPLIED FOR OIFFERENCES BETWEEN

SOARCE EVENTS AND ELDENTS, BUT LIMITED TO -.4 TO +.4

\begin{tabular}{|c|c|c|c|c|c|}
\hline $\begin{array}{l}\text { PARAME } \\
3.06\end{array}$ & $\begin{array}{c}\text { ETERS FOR } \\
0 \quad 5.00\end{array}$ & $\underset{3.00}{\text { FAULT }}$ & $\begin{array}{r}\text { SHAPE: } \\
5.00\end{array}$ & LUES, & IN KILOMETERS \\
\hline $\begin{array}{c}\text { SOURCE } \\
0 \\
0 \\
0 \\
0 \\
0 \\
0 \\
0 \\
0 \\
0 \\
0 \\
0 \\
0 \\
0 \\
0 \\
0\end{array}$ & $\begin{array}{c}\text { Events } \\
1 \\
2 \\
3 \\
4 \\
5 \\
6 \\
7 \\
8 \\
9 \\
10 \\
11 \\
12 \\
13 \\
14 \\
15\end{array}$ & $\begin{array}{l}34.5000 \\
34.5000 \\
34.5000 \\
34.5000 \\
34.5000 \\
34.5600 \\
34.5600 \\
34.5600 \\
34.5600 \\
34.5600 \\
34.5000 \\
34.5000 \\
34.5000 \\
34.5000 \\
34.5000\end{array}$ & $\begin{array}{l}\text { LOCATION } \\
118.5437 \\
118.5220 \\
118.5600 \\
118.4780 \\
118.4563 \\
118.5437 \\
118.5220 \\
118.5600 \\
118.4780 \\
118.4563 \\
118.5437 \\
118.5220 \\
118.5600 \\
118.4780 \\
118.4563\end{array}$ & $\begin{array}{l}1.00 \\
1.00 \\
1.60 \\
1.60 \\
1.00 \\
3.00 \\
3.60 \\
3.60 \\
3.00 \\
3.00 \\
5.80 \\
5.60 \\
5.00 \\
5.00 \\
5.00\end{array}$ & $\begin{array}{c}\text { MOMENT } \\
0.100000 E+21 \\
0.100000 E+21 \\
0.100000 E+21 \\
0.100000 E+21 \\
0.100000 E+21 \\
0.100000 E+21 \\
0.100000 E+21 \\
0.100000 E+21 \\
0.100000 E+21 \\
0.100000 E+21 \\
0.100000 E+21 \\
0.100000 E+21 \\
0.100000 E+21 \\
0.100000 E+21 \\
0.100000 E+21\end{array}$ \\
\hline
\end{tabular}

-ATION: 1 SLAT- 34.5451 SLON 118.5000

\begin{tabular}{|c|c|c|c|c|c|c|c|c|c|c|c|c|c|}
\hline $\begin{array}{l}\text { ELEM } \\
\text { ELEM } \\
\text { ELEM } \\
\text { ELEM } \\
\text { ELEM } \\
\text { ELEA } \\
\text { ELEM } \\
\text { RING: }\end{array}$ & $\begin{array}{l}2 \\
3 \\
4 \\
5 \\
6 \\
7 \\
8 \\
2\end{array}$ & 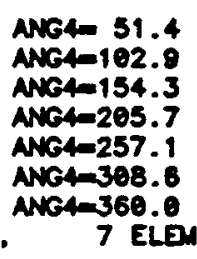 & 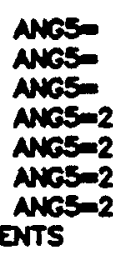 & $\begin{array}{l}90.0 \\
90.0 \\
90.0 \\
270.0 \\
270.0 \\
270.0 \\
273.4 \\
\text { RADIus }\end{array}$ & 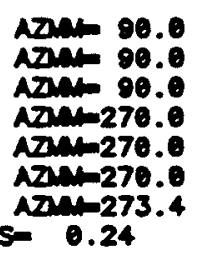 & $\begin{array}{l}\text { EVENT } \\
\text { EVENT } \\
\text { EVENT } \\
\text { EVENT } \\
\text { EVENT } \\
\text { EVENT } \\
\text { EVENT }\end{array}$ & $\begin{array}{l}0 \\
0 \\
0 \\
0 \\
0 \\
0 \\
0\end{array}$ & $\begin{array}{l}8 \\
8 \\
8 \\
8 \\
8 \\
8 \\
8\end{array}$ & $\begin{array}{l}\text { DIS= } 0.24 \\
\text { DIS- } 0.24 \\
\text { DIS= } 0.24 \\
\text { DIS= } 0.24 \\
\text { DIS= } 0.24 \\
\text { DIS= } 0.24 \\
\text { DIS= } 0.24\end{array}$ & $\begin{array}{l}\text { DTIMP- }-0.02 \\
\text { DTIMP } 0.01 \\
\text { DTIMP= } 0.03 \\
\text { DTIMP= } 0.03 \\
\text { DTIMP } 0.01 \\
\text { DTIMP=-0.02 } \\
\text { DTIMP=- } 0.03\end{array}$ & $\begin{array}{l}34.5000 \\
34.5000 \\
34.5000 \\
34.5000 \\
34.5000 \\
34.5000 \\
34.5000\end{array}$ & $\begin{array}{l}118.4979 \\
118.4974 \\
118.4989 \\
118.5011 \\
118.5026 \\
118.5020 \\
118.5000\end{array}$ & $\begin{array}{l}2.85 \\
3.85 \\
3.22 \\
3.22 \\
3.05 \\
2.85 \\
2.76\end{array}$ \\
\hline $\begin{array}{l}\text { ELEM } \\
\text { ELEM } \\
\text { ELEM } \\
\text { ELDM } \\
\text { ELEM } \\
\text { ELEM } \\
\text { ELDM } \\
\text { ELEM } \\
\text { ELEM } \\
\text { ELD }\end{array}$ & $\begin{array}{r}9 \\
10 \\
11 \\
12 \\
13\end{array}$ & $\begin{array}{l}\text { ANG4- } 25.7 \\
\text { ANG4-51 } 51.4 \\
\text { ANG4- } 77.1 \\
\text { ANG4=102.9 } \\
\text { ANG4=128.6 } \\
\text { ANG4-154.3 } \\
\text { ANG4-188.6 } \\
\text { ANG4-205.7 } \\
\text { ANG4-231.4 } \\
\text { ANG4-257.1 }\end{array}$ & 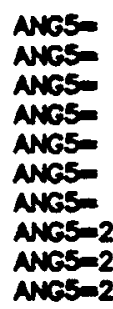 & $\begin{array}{l}90.0 \\
90.0 \\
90.0 \\
90.0 \\
90.0 \\
96.8 \\
270.0 \\
270.0 \\
270.0\end{array}$ & 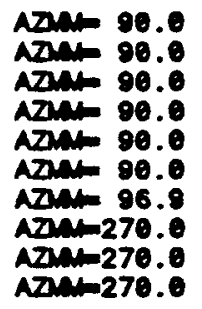 & $\begin{array}{l}\text { EVENT } \\
\text { EVENT } \\
\text { EVENT } \\
\text { EVENT } \\
\text { EVENT } \\
\text { EVENT } \\
\text { EVENT } \\
\text { EVENT } \\
\text { EVENT } \\
\text { EVENT }\end{array}$ & $\begin{array}{l}0 \\
0 \\
0 \\
0 \\
0 \\
0 \\
0 \\
0\end{array}$ & $\begin{array}{l}8 \\
8 \\
8 \\
8 \\
8 \\
8 \\
8 \\
8 \\
8\end{array}$ & $\begin{array}{l}\text { DIS- } 0.47 \\
\text { DIS- } 0.47 \\
\text { DIS- } 0.47 \\
\text { DIS= } 0.47 \\
\text { DIS- } 0.47 \\
\text { DIS= } 0.47 \\
\text { DIS= } 0.47 \\
\text { DIS- } 0.47 \\
\text { DIS- } 0.47 \\
\text { DIS- } 0.47\end{array}$ & $\begin{array}{l}\text { DTIMP=-0.06 } \\
\text { DTIMP=-0.04 } \\
\text { DTIMP=-0.01 } \\
\text { DTIMP }=0.02 \\
\text { DTIMP= } 0.05 \\
\text { DTIIMP= } 0.07 \\
\text { DTIMP= } 0.07 \\
\text { DTIMP= } 0.07 \\
\text { DTIMP= } 0.05 \\
\text { DTIMP }=0.02\end{array}$ & $\begin{array}{l}34.5000 \\
34.5000 \\
34.5000 \\
34.5000 \\
34.5000 \\
34.5000 \\
34.5000 \\
34.5000 \\
34.5000 \\
34.5000\end{array}$ & $\begin{array}{l}118.4978 \\
118.4960 \\
118.4950 \\
118.4950 \\
118.4960 \\
118.4978 \\
118.5006 \\
118.5022 \\
118.5040 \\
118.5050\end{array}$ & $\begin{array}{l}2.57 \\
2.70 \\
2.89 \\
3.11 \\
3.30 \\
3.43 \\
3.47 \\
3.43 \\
3.30 \\
3.11\end{array}$ \\
\hline
\end{tabular}


FLEX 19 ANG4-282.9 ANG5-270.0 AZML270.0 ELEM 20 ANG4-308.6 ANG5-270.0 AZ1ML270.0 FLEM 21 ANG4-334.3 AKC5 270.0 AZ1MK270.0 EN 22 ANG4 360.0 ANGS 273.4 AZMM 273.4 . NG: 3, 14 ELENENTS RNDIUS= 0.47

ELEM 23 ANG4 18.0 ANGS= 90.0 AZMK 90.0 ELEM 24 NGG 36.0 NGS- 90.0 NZML 90.0 ELEU 25 ANG4 54.0 NCS 90.0 AZMN 90.0 ELEI 26 ANC4 72.0 NGSS 90.0 AZIN 90.0 ELE 27 ANG4 90.0 NGS 90.0 AZN 90.0 ELEM 28 MNG4-108.0 ANC5- 90.0 AZNM 90.0 ELEN 29 NGC4128.0 NKC5 90.0 AZN 90.0 ELEY 30 NCA144. ANCS 90.0 ATM 90.0 ELEM 31 NG4-162.0 NCS 00.0 NZML 90.0

ELEN 32 NC4180.0 NCS 96.0 NZM 98.0 ELEX 33 NMG4-188.0 ANOS-270.0 NZMW=270.0 ELEM 34 NG4-216.0 NGS-270.0 AZMm-270.0

ELEN 35 NGS4-234.0 NCS-270.0 ATMm270.0

ELEM 36 NG4-252.0 NaS-270.0 NDML270.0

ELEI 37 NGC4270.0 NKCS=270.0 AZMm-270.0

ELEM 38 ANG4-288.0 ANCS-270.0 AZNM-270.0

ELBM 39 NC4-308.0 NKS-270.0 AZMm270.0

ELEM 40 NG4m324.0 NMOS-270.0 NZML270.0

ELEN 41 NKC4-342.0 NMCS-270.0 AZMN270.0

ELDI 42 NMC4-360.0 NMS-273.4 ADMm-273.4

RING: 4, 20 ELEMENTS RADIUS- 0.70

ELEN 43 ANG4 13.3 NKGS 90.0 ADMW 90.0

ELEN 44 ANG4- 26.7 ANG5 90.0 ATMK 90.0

ELEN 45 ANC4 40.0 ANGS- 90.0 AZN 90.0

ELD 46 NKC4 53.3 AKCS 90.0 AZMr 90.0

ELDN 47 NC4- 68.7 ANG5= 90.0 ATM 90.0

ELEN 48 ANG4 80.0 NKC5 90.0 NZML 90.0

ELD 49 NNE4- 83.3 ANG5- 90.0 NTMm 90.0

ELE 50 ANC4=106.7 ANC5 90.0 AZM 90.0

ELEU 51 ANC4-120.0 AMES- 00.0 AZMK 90.0

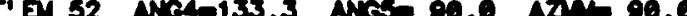

II 53 NMC4-146.7 AMS5 90.0 N7Mn 90.0

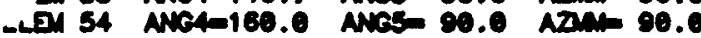

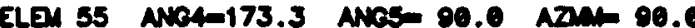

ELD 58 No4-188.7 NGS-270.0 ATMm270.0

ELEN 57 NNG4-260.0 ANES-270.0 AZN-270.0

ELD 58 NWC-213.3 ANGS-270.0 AZNW-270.0

ELE 59 NC4-226.7 MNG-270.0 ATM-270.0

ELD 60 NNG4-240.0 ANG5-270.0 ATMW-270.0

ELEM 61 NC4-253.3 NMCS-270.0 NZML270.0

ELD 62 NKC4-288.7 NGS-270.0 NDMm-270.0

ELEN 63 ANG4-280.0 ANG5-270.0 ATM-270.0

ELEN 64 NNG4293.3 ANGS-270.0 NDM-270.0

ELEN 65 NGC4-306.7 ANG5-270.0 AZML270.0

ELEM 66 NCA4-320.0 NAS-270.0 AZNM-270.0

ELEN 67 NGC4-333.3 NaS-270.0 NZM-270.0

ELDI 68 NGS4-346.7 MNOS-270.0 NZM-270.0

ELEN 69 ANG4 360.0 ANGS-273.4 AZMM-273.4

RING: 5,27 ELEMENTS RNDIUS- 0.93

ELEM 70 ANGA 10.0 NKOS 90.0 ATMm 90.0

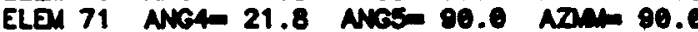

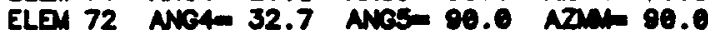

EVENT

EVENT

EVENT

EVENT

EVENT

EVENT

EVENT

EVENT

EVENT

EVENT

EVENT

EVant

EVENT

EVENT

EVENT

EVaNT

EVanT

Evart

VENT

Event

Event

EVENT

EVeNT

EVENT

EVENT

EVENT

EVENT

EVENT

EVENT

EVENT

Event

EVart

Evar

Event

EVENT

EvanT

Evant

EVITT

EVENT

EVENT

EVENT

EVENT

EVENT

EVENT

EVENT

EVENT

EVENT

EVENT

EVENT

Event

EverT

EVENT $\begin{array}{ll}8 & \text { DIS }=0.47 \\ 8 & \text { DIS }=0.47 \\ 8 & \text { DIS }=0.47 \\ 8 & \text { DIS }=0.47\end{array}$

TIMP-0.01

DTIMP-0.04

DTIMP-0.08

34.5000

34.5000

34.5000

118.5858

118.5040

118.5022

118.5000

2.89

2.70

2.57

2.53

2.33

2.43

2.59

2.78

3.00

3.22

3.41

3.57

3.67

3.70

3.67

3.57

3.41

3.22

3.00

2.78

2.59

2.43

2.33

2.30

2.69

8 DIS= 0.93 DTINP-0.11

8 DIS 0.93 DTIMP- 0.10

8 DIS- 0.93 DTIMP- 0.08

8 DIS- 0.93

DTINP=-0.08

DIS= 0.93 DTIMP-0.03

8 DIS- 0.93 DTINP-0.60

8 DIS= 0.93

8 DIS- 0.93

8 Dise 0.03

DTIP 0.03

8 DIS 0.93

8 DIS- 0.83

8 DIS- 0.03

DTINP 0.06

DTINP 0.08

DTIN 0.11

8 DIS- 0.03

- DIS- 0.03

8 DIS- 0.83

DTINP= 0.13

DTINP 0.14

DTINP= 0.15

DTINP= 0.15

DIS- 0.83

8 DIS- 0.93

8 DIS- 0.93

8 DIs= 0.83

8 DIS- 0.83

8 DIS= 0.93

DIS 0.93

DIS- 0.93

8 DIS= 0.93

DIS- 0.83

DIS= 0.83

8 DISm 0.93

DTIN 0.14

DTINP 0.11

DTIMP 0.09

OTIMP 0.06

DTINP 0.03

DTIM $=-0.00$

DTIMP-0.03

DT IMP -0.06

DTINP=-0.08

OTINP -0.10

DTINP=-0.11

DTINP -0.12

34.5000

34.5000

34.5000

34.5000

34.5000

34.5000

34.5800

34.5600

34.5000

34.5600

34.5800

34.5000

34.5800

34.5000

34.5000

34.5000

34.5000

34.5000

34.5000

34.5000

34.5000

34.5000

34.5600

34.5000

34.5000

34.5000

34.5000

118.4977

118.4854

118.4035

118.4919

118.4907

118.4960

118.4890

118.4903

118.4912

118.4928

118.4944

118.4965

118.4988

118.5012

118.5035

118.5058

188.5074

118.5088

118.5097

118.5101

118.5100

118.5093

118.5081

118.5065

118.5045

118.5023

118.5800

2.17

2.29

2.44

2.63

2.84

3.05

3.27

3.47

.84

3.78

3.88

3.93

3.93

3.88

3.64

3.47

3.27

3.05

2.84

2.63

2.44

2.29

2.17

2.09

2.07

1.86

1.92

2.02 
ELEN 73 ANG40 43.6 ANG5 90.0 AZML 90.0

ELEN 74 ANG4 54.5 NHC5 90.0 AZML 90.0

-I.D 75 ANC4 65.5 ANCS 90.0 AZML 90.0

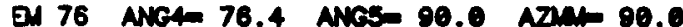

-LD 77 NG4 87.3 NKCS- 90.0 NZML 90.0

ELa 78 ANG4 98.2 ANCS 90.0 AZML 90.0

ELEX 79 NKC4-188.1 NKC5 90.0 AZML 90.8

ELEM 80 ANG4-120.0 NNES 90.0 AZML 90.0

ELEM 81 ANG4-130.9 ANGS- 90.0 NZIN 80.0

ELEN 82 ANG4-141.8 ANCS 90.0 NZMN 00.0

ELEM 83 ANG4-152.7 ANGS 00.0 AZML 00.0

ELE 84 ANC4-163.6 ANCS- 90.0 ATML 80.0

ELEU 85 ANG4-174.5 ANCS- 90.0 ATMm 90.0

ELEN 86 ANG4-185.5 ANGS-270.0 AZML-270.0

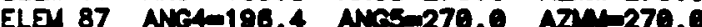

ELE 88 ANC4-207.3 NKCS-270.0 ATML270.0

ELEM 89 ANC4-218.2 ANGS-270. NZM-270.0

ELO 98 NCS-220.1 ANC6-270. ATMr270.

ELEM 91 AKC4-240.0 ANES-270.0 AZMm270.0

ELEN 92 NCA-250.9 NWS-270.0 ATN-270.8

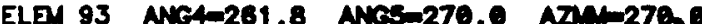

ELEM 94 NC4-272.7 NCS 270.0 NZMm-270.0

ELEM 95 NKC4-283.6 NWS-270.0 NZM-270.0

ELD 96 NCA-294.5 AMES-270.0 ATML270.0

ELEN 97 NKA-305.5 NOS-270.0 AZNM-270.0

ELEN 98 NNC4-316.4 ANOS-270.0 AZNM-270.0

ELE 99 NC4-327.3 AMCS-270.0 . ATML270.0

ELD100 NNC4-338.2 NAS-270.0 AZML-270.0

ELEN181 NCA-349.1 ANES-270.0 AZMM-270.0

ELEMI02 ANC4-360.0 ANOS-273.4 AZM-273.4 RING: 6, 33 ELEUENTS RADIUSO 1.18

ELEN103 NNG4 9.2 ANGS 90.0 NZMK 90.0

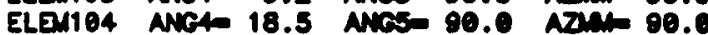

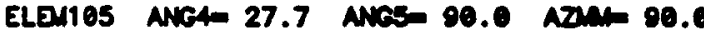

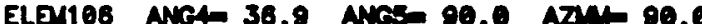

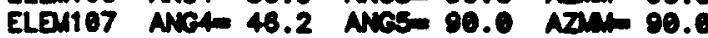

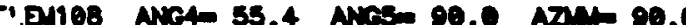

EM109 Mre4 64.6 ANes 90.0 NZMm 90.0

LDN110 NAC4 73.8 ANoS 90.0 AZM 90.0

ElD111 ANC4 83.1 Mres- 09.9 Aan 90.0

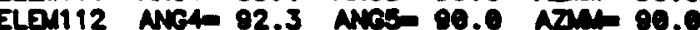

ELDM113 ANC4m101.5 ANGS 90.0 NZML 90.0

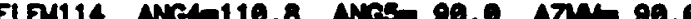

ELDM1 15 NGA-120.0 NKS 90.0 NZM 90.0

ELON116 ANG4-129.2 ANBS 90.0 AZML 90.0

ELE117 NC4-138.5 ANC6- 90.0 ATML 90.0

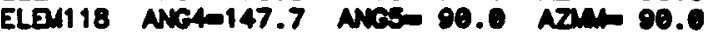

ELEM119 ANC4-156.9 ANES 90.0 AZML 90.0

ELDI20 ANC4=166.2 ANCS- 90.0 ATM 90.0

ELEN121 ANC4-175.4 NKSS 80.0 AZNL 80.0

ELEN122 ANG4-184.6 ANCS-270. AZNM-270.0

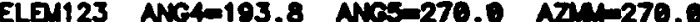

ELEN124 NW4-203.1 ANOS-270.0 NTNm270.0

ELaN125 ANG4-212.3 NMS-270.0 NTMm270.

ELEM128 NGG4221.5 MNCS-270.0 ATML279.0

ELEM127 ANG4-230.8 ANGS-270.0 AZM-270.0

ELEM128 ANG4-240.0 ANGS-270.0 ATMN-270.0

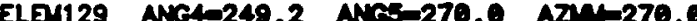

ELEN130 ANG4-258.5 ANCS-270.0 AZMr-270.0
EVENT

EVET

EVENT

EVENT

EVENT

EVENT

EVENT

EVENT

EVENT

EVENT

GVaN

EVENT

EVENT

EVENT

EVEVT

EVENT

Event

EVENT

EVENT

EVENT

EVEN

EVant

EVENT

EVENT

EVENT

EVENT

EVET

EVENT

EVENT

EVENT

EVENT

EVENT

EVET

EVENT

EVENT

EveNT

Evan

EVaNT

EVENT

EVENT

EVENT

EVENT

EVENT

EVaNT

Evart

EVENT

EVENT

Event

EVENT

EVINT

EVENT

EVENT

EVENT

EVENT$$
13
$$

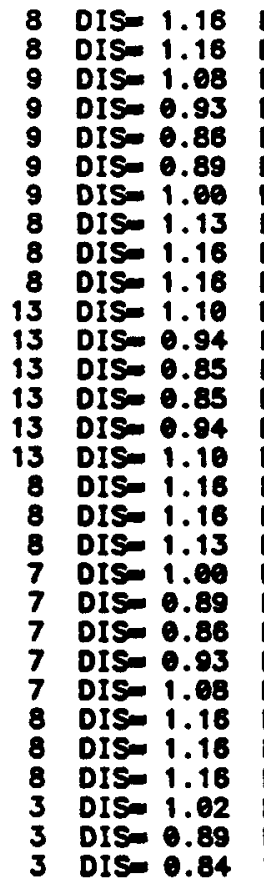

DTIMP $=0.09 \quad 34.5000$ DTIMP-0.07 34.5000 DTIMP=- $0.13 \quad 34.5000$ DTIVP-0.19 34.5690 DTIMP-0.07 34.5000 DTIMP- $0.04 \quad 34.5000$ DTIM=-0.01 34.5600 DTIMP $=0.11 \quad 34.5000$ DTINP $0.14 \quad 34.5600$ DTIIP $0.16 \quad 34.5000$ DTINP=-0.18 34.5000 DTIMP $0.15 \quad 34.5600$ DTIMP $-0.14 \quad 34.5000$ DTIMP- $0.14 \quad 34.5600$ DTIMP=0.15 34.560 DTIMP $-0.18 \quad 34.3800$ DTINP $0.16 \quad 34.5000$ DTIP $0.14 \quad 34.5000$ DTINP= 0.1134 .5000 DTINP-0.e1 34.5600 DTIMP-0.04 34.5000 DTIM $=0.07 \quad 34.5000$ DTIMP $=0.1034 .5000$ OTIMP-0.13 34.5000 DTIMP $-0.07 \quad 34.5000$ DTINP=-0.09 34.5000 DTIMP -0.1134 .5000$ OTIMP= $0.09 \quad 34.5000$ DTIMP= $6.08 \quad 34.5900$ DTIMP 0.07
118.4913 118.4897 118.4885 118.4877 118.4874 118.4875

118.4881

118.4890

118.4004

118.4922

118.4942

118.4984

118.4088

118.5012

118.5036

118.3058

118.5078

118.5808

118.5160

118.5110

118.5125

118.5120

118.5123

118.5115

118.5103

118.5087

118.5060

118.5947

118.5024

118.5600
2.16

2.33

2.52

2.73

2.94

3.17

3.38

3.58

3.78

3.91

4.03

4.11

4.18

4.18

4.11

4.03

3.91

3.58

3.17

2.94

2.73

2.52

2.33

2.16

2.02

1.92

1.88

1.84

1.63

1.68

1.77

1.89

2.04

2.21

2.41

2.61

2.83

3.86

3.28

3.49

3.69

3.88

4.04

4.17

4.28

4.35

4.38

4.38

4.35

4.28

4.17

4.04

3.88

3.60

3.49

3.28 
ELEM131 ANG4-267.7 MGC5-270.0 AZML270.0 ELEN132 ANG4-276.9 ANG5 270.0 AZMm270.0 ANC4-286.2 ANC5-270.0 ATMh270.0 3134 ANG4-295.4 NNG5-270.0 AZM-270.0 EN135 ANG4-304.6 ANG5-270.0 AZN=270.0 ELEM136 NGA-313.8 NGS-270.0 AZML270.0 ELEN137 NGC4-323.1 NGS-270.0 AZMM-270.0 ELEN138 ANG4-332.3 NCS-270.0 AZNM-270.0 ELEM139 ANC4-341.5 MCOS 270.0 ATM 270.0 ELEN140 ANG4-350.8 MVE-270.0 ATML-270.0 ELEM141 ANG4-360.0 NGS-273.4 AZM 273.4 RING: 7, 39 ELENENTS RNDIUS= 1.39

ELEMT42 NNG4 7.8 MKOS 90.0 NZMM 90.0 ELEN143 NG4 15.7 ANES 90.0 NTWL 90.0 ELEM144 ANG4- 23.5 ANGS 90.0 AZN 90.0 ELDM145 ANG4- 31.3 ANGS- 00.0 AZNW 00.0 ELEM146 NG4 39.1 MGS- 90.0 AZM 00.0 ELEN147 NKG4 47.0 NWES 00.0 AZN 00.0 ELEM148 ANG4 54.8 NACS 90.8 AZM 90.0 ELEU149 ANG4 62.6 NCS- 90.0 ATML 90.0 ELEA150 ANC4 70.4 ANCS- 90.0 ATMN 90.0 ELEN151 ANC4 78.3 ANCS- 90.0 AZM 90.0

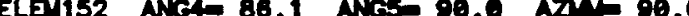
ELEN153 ANC4= 93.9 NNGS- 90.0 AZML 90.0 ELEM154 ANC4-101.7 ANCS 90.0 AZM 90.0

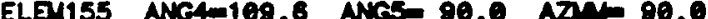
ELEM156 ANG4-117.4 ANG5 90.0 NZML 90.0 ELEA157 ANG4-125.2 ANG5 90.0 AZN 90.0 ELEU158 ANG4-133.0 NNG5- 00.0 NZM 90.0 ELEMI59 ANG4-140.0 ANGS- 90.0 NZMN 90.0 ELEM160 ANG4-148.7 ANCS- 90.0 AZMN 90.0 ELEM161 NG4-158.5 MCS- 80.0 NTMK 80.0 ELEN162 NG4-164.3 NGSE 90.0 AZM 90.0 ELEN163 ANG4-172.2 NMGS 90.0 ANMW 90.0

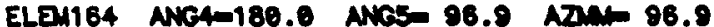
ELEN165 ANG4-187.8 ANGS-270.0 AZMMm270.0 -i.eN166 NNG4-195.7 ANGS-270.0 NZNM-270.0 1187 NC4-203.5 NOS-270.0 N7M-270.0 -LEN168 ANG4-211.3 ANG5-270.0 NZML270.0 ELLE169 ANC4-219.1 ANGS-270.0 AZMM-270.0 ELDA170 ANG4=227.0 ANCS-270.0 ATML270.0 ELEN171 ANG4=234.8 ANG5m270.0 AZML=270.0 ELDN172 ANG4-242.6 ANCS-270.0 AZNM-270.0 ELD173 ANC4-250.4 ANCS-270.0 NTMU270.0 ELEM174 ANG4-258.3 ANCS-270.0 AZMM270.0 ELDM175 ANG4=266.1 ANGS=270.0 AZTM-270.0 ELEM176 ANC4-273.8 ANGS-270.0 ATMW270.0 ELEM177 ANG4-281.7 AMCS-270.0 AZML-270.0 ELEM178 ANC4-289.6 NCS-270.0 AZNW270.0 ELEN179 ANG4=297.4 ANGS-270.0 AZMN270.0 ELEM180 NGA-305.2 NGS-270.0. ATMm-270.0 ELEM181 ANC4-313.0 NNES-270.0 ATML270.0 ELEM182 ANG4-320.9 NGES-270.0 ATMN270.0 ELEN183 NGA-328.7 NGS-270.0 AZML270.0 ELEM184 ANG4-336.5 NOS-270.0 ADMW-270.0 ELEM185 NG4-344.3 NWES-270.0 ATMW270.0 ELEM186 ANG4-352.2 NVES-270.0 ATMM-270.0 ELDIIB7 ANG4-360.0 ANCS-273.4 AZNW-273.4 RING: 8, 46 ELEMENTS RADIUS- 1.61

$\begin{array}{ll}\text { EVENT } & 0 \\ \text { EVENT } & 0 \\ \text { EVENT } & 0 \\ \text { EVENT } & 0 \\ \text { EVENT } & 0 \\ \text { EVENT } & 0 \\ \text { EVENT } & 0 \\ \text { EVENT } & 0 \\ \text { EVENT } & 0 \\ \text { EVENT } & 0 \\ \text { EVENT } & 0\end{array}$

EVent EVENT EVenT

EVerT

EVENT

EVENT

EVENT

EVent

EVarT

EVENT

EVENT

EVENT

EVENT

EVENT

EVENT

EVENT

EVENT

EVENT

EVENT

EVENT

EVarT

EVENT

EVavt

EVEN

EVaNT

EVENT

EVaNT

EVENT

EVENT

EVENT

EVENT

EVENT

EVENT

EVENT

EVENT

EVENT

EVENT

EVENT

Evant
7 DIS- 0.64

7 DIS= 0.67

7 DIS= 0.78

7 DIS $=0.97$

7 DIS- 1.18

8 DIS- 1.36

3 DIS= 1.22

3 DIS- 1.01

3 DIS- 0.81

3 DIS- 0.87

3 DIS- 0.81

3

DIS= 0.46

DIS= 0.62

3 DIS- 0.83

3 DIS- 1.04

DIS 1.22

DIS 1.23

DISe 1.17

DIS 0.95

DIS= 0.74

9 DIS- 0.55

DIS $=0.43$

DIS $=0.43$

DIS= 0.35

DIS- 0.74

DIS= 0.95

DIS- 1.17

14 DIS= 1.23

14 DIS- 1.22

13 DIS- 1.04

13 DIS-

13 DIs- 0.62

13 DIS- 0.46

13 DIS- 0.39

13 DIS- 0.46

13 DIS- 0.62

13 DIS- 0.83

13 DISn 1.04

12 DIS- 1.22

12

DIS= 1.23

DIS- 1.17

DIS- 0.95

7

$5=0.74$

7 DIS= 0.55

7 DIS= 0.43

DIS= 0.43

DIS= 0.55

DIS- 0.74

DIS- 0.95

DIS- 1.17

DIS. 1.23

DIS- 1.22

DIS- 1.04

DIS 0.83

DIS- 0.62

3 DIS 0.46

3 DIS- 0.30
OTIMP -0.04 DTIMP $=0.07$ DTIMP $=0.10$ DTIMP- 0.13 DTIMP-0.16 DTINP-0.09 DTIMP 0.10 DTIMP= 0.08 DTIMP 0.06 DTIMP 0.05 DTINP= 0.05

DTIMP 0.03 DTIMP 0.04 DTIMP 0.05 OTIMP 0.07 DTIMP -0.03 DTIMP -0.00 OTIMP -0.17 DTINP=0.14 DTINP-0.11 DTINP -0.08 DTIMP-0.05 DTIMP $=0.02$ DTINP 0.01 DTIN 0.04 DTIMP 0.07 DTIMP 0.09 DTIMP-0.20 DTIMP -0.18 DTIMP-0.09 DTIMP -0.08 DTIMP-0.07 DTIMP-0.07 DTIMP -0.07 DTIMP -0.07 DTIMP-0.07 DTIMP-0.08 DTIMP-0.09 DTIMP -0.18 DTIMP -0.20 DTIMP 0.09 DTIMP 0.07 DTIMP 0.04 DTIMP 0.01 DTINP-0.02 OTIM $=0.05$ DTIMP -0.08 DTIMP -0.11 DTIMP-0.14 DTIMP -0.17 DTIIP- 0.00 DTIMP-0.03 DTIMP 0.07 DTIMP 0.05 DTIMP 0.04 DTINP= 0.03
34.5000 34.5000 34.5000 34.5000 34.5000 34.5000 34.5000 34.5000 34.5000 34.5000 34.5000

34.5000 34.5000

34.5000

34.5000

34.5000

34.5000

34.5000

34.5600

34.5000

34.5000

34.5600

34.5000

34.5000

34.5000

34.5000

34.5000

34.5000

34.5000

34.5000

34.5000

34.5000

34.5000

34.5000

34.5060

34.5000

34.5000

34.5000

34.5000

34.5000

34.5000

34.5000

34.5000

34.5000

34.5000

34.5000

34.5000

34.5000

34.5000

34.5000

34.5000

34.5000

34.5000

34.5000

34.5000

34.5000

34.5000

18.5151

118.5150

18.5145

118.5136

118.5124

118.5169

118.5081

118.5070

118.5948

118.5024

118.5600

118.4978

18.4953

118.4930 
ELD188 ANG4 6.9 MC5 90.0 ATMK 90.0 FLGM189 ANG4 13.8 NGGS 90.0 AZML 90.0 D190 ANG40 20.8 ANC5 90.0 ATM 00.0 gM191 ANG4 27.7 ANC5- 90.0 ATML 00.0 ELEN192 ANG4= 34.6 AKCSM 90.0 AZML 90.0 ELEN193 AGG4 41.5 ANCS- 90.0 A7ML 90.0 ELEM194 ANG4 48.5 NKC5 90.0 ATML 90.0 ELDU195 ANC4 55.4 NKC5 90.0 AZML 90.0 ELEI196 ANG4 62.3 ANC5 09.0 ATM 09.0 ELPM197 NG4 69.2 NKCS- 90.0 NTML 90.0 ELEN198 NGG4 76.2 NWG5 90.0 NZMM 00.0 ELDM199 ANG4 83.1 NAS- 80.0 NTML 00.0 ELD1280 MG4 98.0 NMS 90.0 ATML 00.0 ELEN201 NGC4 98.9 ANGS 90.0 AZML 90.0 ELg1202 ANG4-103.8 AC5 00.0 ATM 90.0 ELEV203 MG4-110.8 NCS 09.0 ATML 90.0 ELEN204 NG4117.7 ANGS 00.0 AZMK 00.0 ELEV205 ANG4-124.6 ANCS- 09.9 ATM

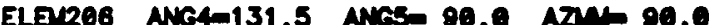
ELE1207 ANG4138.5 NWS- 00.0 NZN 00.0 ELER98 ANG4-145.4 AMBS- 09.9 ATM 90.0 ELER209 NG4-152.3 NKS 00.0 ATML 00.0 ELER10 ANCA159.2 NHES 00.0 A2ML 00.0 ELEV11 ANC4-168.2 ANoS- 09.0 ATM 09.0

ELEN12 ANC4-173.1 ANCS- 90.0 AZML 00.0

ELEN13 ANC4180.0 NNAS- 96.0 AZM 96.8

ELON214 NC4186.9 ANR6-270.0 ATML270.8

ELEL15 ANG4-193.8 NWS-270.0 NZMN-270.0

ELER216 ANG4-280.8 NKS-270.0 ATNm270.6

ELDR17 NG4-207.7 NMES-270.0 ATMm-270.6

ELER18 ANG-214.6 ANES-270.0 NZN-270.0

ELDR19 ANG4-221.5 NMS-270.0 ATMm270.0

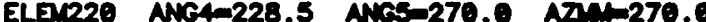

ELDR21 ANG4-235.4 NNGS-270.0 ATMm-270.0

ELEV222 ANC4m242.3 ANCSm270.0 ATMm270.0

ELDR23 NMEA-249.2 NKCS-270.O NTMm270.0

-i.D2224 NMC4-256.2 NNES-270.0 NTM-270.0

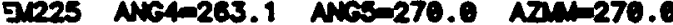

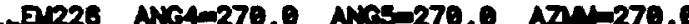

ELER27 ANG4-276.0 NMS-270.0 AZM-270.0

ELER28 ANC4283.8 NAS-270.9 NTML270.9

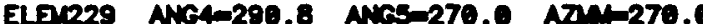

ELEN230 ANG4-297.7 ANCS-270.0 AZMM-270.0

ELEN31 ANC4-304.8 NFS-270.0 NTMm270.0

ELER32 ANC4-311.5 NNeS-270.0 ATM 270.0

ELDK233 NAC4-318.5 NMES-270.0 NZNm-270.0

ELER34 NMC4325.4 MNS-278.9 ATM-270.0

ELER235 NCA-332.3 NNCS-270.0 NTMN270.0

ELE1236 NMC4-339.2 ANCS-270.6 NTN-270.

ELER37 ANG4-346.2 ANCS-270.0 ATMm270.0

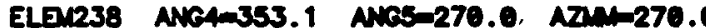

ELDN230 ANC4-360.0 ANBS-273.4 AZNM-273.4

RING: 9, 52 ELEMENTS RADIUS- 1.84

ELER40 NGG 6.2 ANGS 90.0 ATAN 90.0 ELEV241 NG4 12.4 ANCE 90.0 ATM 90.0

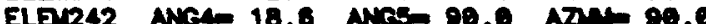
ELER 43 NG4 24.8 ANeS- 00.0 ATM 00.0

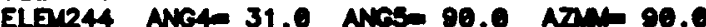

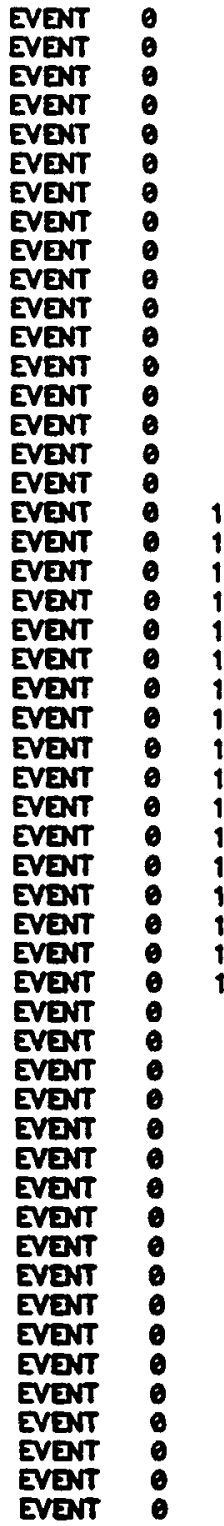

3 DIS 0.28 3 DIS- 0.49 3 DIS 0.71

3 DIS- 0.83

4 Dise 1.09

4 DISE 1.02

DISe 1.01

4 Dise 1.08

9 DIS- 0.94

9 DISo 0.72

9 DIS= 0.50

9 DIS 0.30

9 DIS= 0.18

9 DIS- 0.30

9 DISo 0.50

9 DIS- 0.72

9 DIS- 0.94

14 DIS= 1.08

14 DIS 1.01

14 DIS- 1.02

14 DISE 1.09

13 DIS= 0.93

13 OIS 0.71

13 DISn 0.49

13 DIS- 0.28

13 DIS= 0.18

13 DIS- 0.28

13 DIS- 0.49

13 DIS- 0.71

13 DISm 0.93

12 Dis- 1.09

2 DIS= 1.02

12 DIS- 1.01

12 DIS- 1.08

7 DIS 0.94

7 otse e.72

7 DIS- 0.50

7 DIS 0.30

DIS 0.18

7 DIS- 0.30

DIS- 0.50

DIS 0.72

DIS- 0.94

DIS= 1.08

DIS- 1.01

DIS= 1.02

DIS- 1.09

DIS= 0.93

DIS- 0.71

DIS- 0.49

DIs- 0.28

3 Diso 0.16

OTIMP= 0.01 DIIP 0.02 DTIMP 0.03 DTINPa 0.05 DTIMP -0.05 DTIMP $=0.03$ DTIMP-0.00 DTIM $=0.03$ DTIMP- 0.14 DTIMP 0.11 DTIMP $=0.08$ DTIMP-0.05 DTINP-0.02 DTIMP 0.01 DTINP 0.04 DTIMP 0.07 DTIMP 0.10 ortMp- -18 DTIMP -0.17 DTIMPa-0.15 DTIMP-0.13 DTIMP-0.05 OTIMP -0.04 DTIMP-0.03 DrIMP- -0.03 DTINP-0.03 DTIMP- 0.03 DTINP-0.03 DTIMP-0.04 DTINP=0.05 DTINP -6.13 DTINP-0.15 DTINP -0.17 DTIMP -0.19 DTINP= 0.10 DTIN= 0.07 DTIMP= 0.04 DTIM $=0.01$ DTIM -0.02 OTIMP -0.05 OTIMP=0.08 OTIMP=- 0.11 DTIMP-0.14 DTIMP $=0.03$ DTIMP-0.00 OTIMP-0.03 orIMP=0.85 DTIMP 0.05 DTINP 0.03 DTIM= 0.02 DTIMP 0.01 DTIMP $e .01$

34.5000

$34.5000 \quad 118.4828$

$34.5000 \quad 118.4907$

$34.5000 \quad 118.4886$

$34.5000 \quad 118.4867$

$34.5000 \quad 118.4850$

34.5000118 .4835

$34.5600 \quad 118.4823$

34.5600118 .4813

34.5000118 .4808

$34.5000 \quad 118.4801$

$34.5860 \quad 118.4800$

34.5000118 .4801

34.5000118 .4898

$34.5060 \quad 118.4813$

34.5000118 .4623

$34.5090 \quad 118.483$

34.5000118 .4050

34.5000118 .4867

$34.5060 \quad 118.488$

34.5060118 .4007

34.5000118 .4920

$34.5600 \quad 118.4952$

$34.5000 \quad 118.4078$

34.5000118 .3000

34.5060118 .5024

34.5000118 .5040

$34.5000 \quad 118.5071$

$34.5000 \quad 118.5803$

34.5000118 .5114

34.5600118 .5133

$34.5690 \quad 118.5150$

34.5000118 .5165

$34.5000 \quad 118.5177$

34.5999118 .5187

34.5000118 .5104

34.5090118 .5190

$34.5690 \quad 118.5290$

34.5600118 .5190

34.5969118 .5194

$34.5600 \quad 118.5187$

34.5000118 .517

34.5689 118.5185

$34.5000 \quad 118.5150$

34.5600118 .5133

34.5690118 .5114

$34.5000 \quad 118.5003$

34.5000118 .5071

$34.5090 \quad 118.5948$

$34.5000 \quad 118.5024$

34.5060118 .5800

1.18

1.22

1.37

1.49

1.62

1.78

1.96

2.15

2.35

2.56

2.78

3.80

3.22

3.44

3.65

3.85

4.04

4.22

4.38

4.51

4.63

4.72

4.82

4.84

4.82

4.78

4.72

4.83

4.51

4.22

4.04

3.85

3.65

3.44

3.22

3.60

2.78

2.58

2.35

2.15

1.78

1.62

1.49

1.37

1.28

1.18

1.18

Evart

3 DIS 0.23

DTIMP-0.00

34.5000

118.4978

$34.5000 \quad 118.4952$

DTIM $=0.02$

DTIM $=0.02$

34.5000

34.5000

118.4928

118.4908

118.4804
0.05

0.09

1.85

1.13 
ELEM245 ANG4- 37.2 ANG5- 90.0 AZMK 90.0 ELEN246 ANG4 43.4 NNGS 90.0 ADNL 90.0

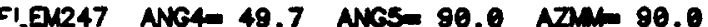
IN248 ANG4 55.9 NCOS= 90.0 AZML 90.0 -EV249 ANG4 62.1 ANGS 80.0 AZNW 90.0 ELDU250 ANG4 68.3 ANG5 90.0 NZML 90.0 ELEN251 ANG4 74.5 ANGS- 90.0 AZM 90.0 ELEN252 ANG4 80.7 ANG5 90.0 AZM 90.0 ELEL253

ELEN254

ELEN255

ELEN256

ELEN257

ELEN258

ELEU259

ELEL260

ELEN26

ELEN262

ELEN263

ELEN28

ELEN265

ELPV26

ELEN267

ELEN268

ELEN69

ELEN27

ELER7

ELEN272

ELEN273

ELE 1274

ELEV275

ELEN27

ELEN277

ELDU278

ELEN279

ELE 280

ELan8

I. D1282

בर283

EN28.

ELER285

ELEN28

$51{ }^{2} 887$

ELAR8:

ELEN289

ELตบ290

ELEN291

ELEN29

ELEN293

ELEN294

ELEN29

ANG4-347.6

ELEN297 ANG4-360.0 ANBS-273.4 ATM-273.4

RING: 10, 58 ELEMENTS RADIUSO 2.08

ELEN298 ANG4- 5.5 ANCS- 90.0 AZMW 90.0 ELER299 ANC4 11.1 AWCS- 80.0 ADML 90.0 ELau360 ANG4 16.6 AMCS 90.0 AZM 90.0 ELEMBO1 NGG 22.2 NGS- 90.0 ATML 90.0 ELEM302 ANG4- 27.7 ANCS- 90.0 ATML 90.0

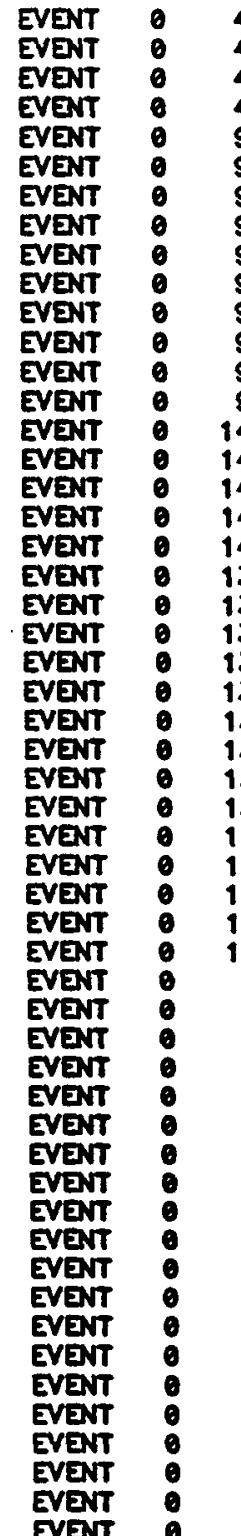

\begin{tabular}{|c|c|}
\hline 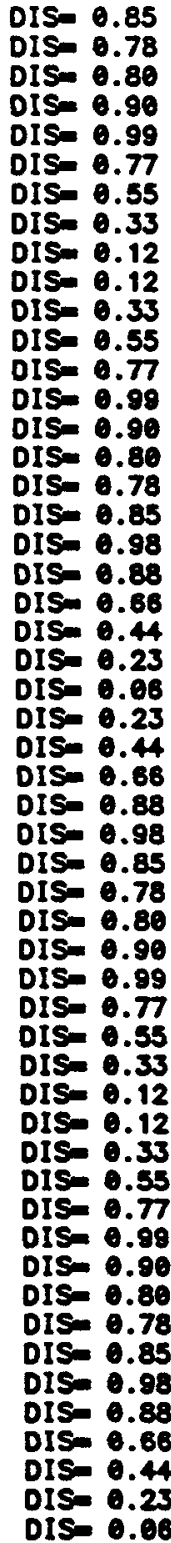 & 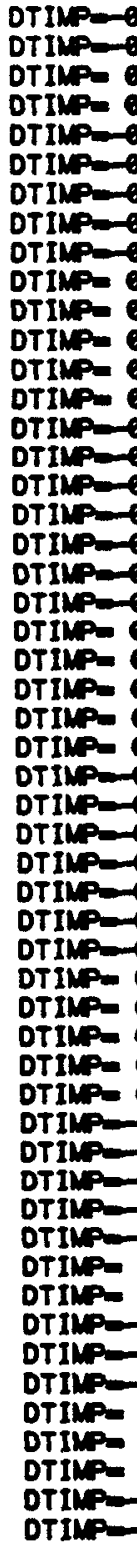 \\
\hline
\end{tabular}

34.5000 34.5000

34.5000

34.5000

34.5000

34.5000

34.5000

34.5000

34.5060

34.5000

34.5090

34.5000

34.5000

34.5000

34.5000

34.5000

34.5000

34.5800

34.5608

34.5000

34.5000

34.5060

34.5800

34.5000

34.5000

34.5800

34.5600

34.5000

34.5000

34.5000

34.5000

34.5000

34.5800

34.5000

34.5000

34.5000

34.5000

34.5000

34.5000

34.5000

34.5000

34.5000

34.5000

34.5000

34.5000

34.5000

34.5000

34.5000

34.5000

34.5000

34.5000

34.5000

198.4864

118.4848

118.4829

118.4814

118.4801

118.4781

118.4784

118.4778

118.4776

118.4778

118.4778

118.4784

118.4781

118.4801

118.4814

118.4829

118.4846

118.4864

118.4884

118.4908

118.4928

118.4952

118.4978

118.5909

118.5024

118.5048

118.5672

118.5694

118.5116

118.5136

118.5154

118.5171

118.5186

118.5198

118.5200

118.5218

118.3222

118.5224

118.5224

118.3222

198.3218

118.5280

118.5188

118.5188

118.5171

118.5154

118.5138

118.5118

118.5094

118.5072

118.5040

118.5024

118.5600

.36

1.50

1.66

1.84

2.03

2.24

2.45

2.67

.89

3.11

3.33

3.76

3.97

4.18

4.34

4.50

4.64

4.77

4.87

4.95

5.01

5.05

5.08

5.05

5.01

4.85

4.87

4.77

4.64

4.50

4.34

4.16

3.97

3.78

3.55

33

3.1 !

2.89

2.67

2.45

2.24

.03

1.84

1.68

1.36

1.23

1.13

1.85

0.89

0.04

0.72

0.78

0.81

$0.88 /$

0.97 
ELEN3O3 ANG4- 33.2 ANG5 90.0 AZMM 90.0 ANG4 38.8 ANCS- 90.0 ATM 90.0

ELEuzes 5u306 $-91307$

ELEU308

ELEM309

ELEU310

ELEN311

ELEM312

ELEUS13

ELEM314

ELEN315

ELEV316

ELEU317

ELEK318

ELE1319

ELEN320

ELEX321

ELEU323

ELEN324

ELEM32:

ELEU326

ELEM327

ELEU328

ELEM329

ELgu33:

ELEL33

ELEL332

ELEM33.

ELPV334

ELEu335

ELEV336

ELE1337

ELEN33:

ELDU339

$-1.31340$

9u341

ELEN345

ELE1344

ELg1345

ELEN346

ELEL347

ELEV348

ELEN349

ELEV350

ELEN35

ELEu352

ELE1353

ELEU35

ELDU355

ELDM358

ELEU357

ELDU358

ELEM360

ELEN36

ELEM362
ANG4

ANG4 49.8

ANG4 55.4

ANG4 60.8

ANG4- 66.5

ANo4 72.0

Nute 77.5

ANes- 83.

NGG4 94.2

Nub4- 98.7

NG4-105.2

NGef-1 18.8

ANG4-118.

NOA4-121.8

Nuc4-127.4

Anc4-132.8

ANG4-138.5

ANC4-144.

ANo4-149.5

NC4-155.1

ANC4=160.6

ANG4168.2

ANG4=171.7

ANe4=177.2

ANCA $=182.8$

NiG4-188.3

ANC4-193.8

ANC4-198.4

Nac4-204.9

ANC4-210.5

ANC4 216.8

ANG4-221.5

ANG4-227.

Mus4-232.6

ANa4-238.2

NGS 243.7

NG4-249.2

NG4-254.8

ANC4-260.3

ANG4 265.8

ANG4=271.4

ANG4-276.9

ANC4-282.5

ANG4-288.0

Nic4-203.5

ANG4-299.

ANG4-304.6

ANG4-310.2

ANC4-315.7

ANG4-321.2

ANG4 -332.3

ANG4 337.8

NMA-343.4

NVG -348.9

ANG4 354.5

ANG4-360.0
NAS- 90.0 AZM 90.0

NAC5- 90.0 AZML 90.0

NCS- 90.0 AZMm 90.0

NWES- 90.0 AZMK 90.0

ANC5- 90.0 AZML 90.0

NCS- 90.0 ATM 90.0

ANeS- 90.0 AZML 90.0

NMES- 90.0 AZM 90.0

MNES 90.0 ATML 80.0

ANCS 90.0 AZML 00.0

ANBS 98.0 AZMK 90.0

NMES 90.0 ATn 90.0

ANGS 90.0 AZM 90.0

Muse 90.0 Nank 90.0

ANas 90.0 ATM 90.0

AMES 90.0 ATN 90.0

ANCS= 00.0 AMN 90.0

ANCS 80.0 AZML 90.0

NCS- 90.0 AZMK 90.0

AMcs 90.0 AzN 90.0

ANGS= 90.0 AZML 90.0

NACS= 90.0 NZMW 90.0

ANCS 90.0 ATML 90.0

ANCS= 00.0 AZMN 90.0

ANC5-270.0 AZML-270.0

ANes-270.0 ATML270.0

MVS-270.0 AZMm-270.8

AMCS-270.8 ADML270.0

ANos-270.0 ATM 270.0

ANCS $=270.0$ AZML-270.0

ANCS-270.0 AZML-270.0

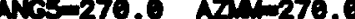

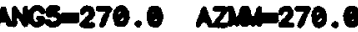

ANG5-270.0 AZMس-270.0

AN5-270.0 ATNme270.0

NMSS-270.0 NUML-270.0

ANCS-270.0 AZMW-270.0

AMES 270.0 ATIM 270.0

ANCS-270.0 AZMN-270.0

ANes-270.0 AZM-270.0

ANC6m270.0 NTML270.0

ANGS-270.0 AZMN-270.0

NMS-270.0 NTMm270.0

NMGS-270.0 AZML-270.0

ANCS 270.0 AZM 270.0

NMS 278.0 ATM 270.0

NMCS-270. AZML-270.0

ANaS-270.0 AZMM-270.0

ANo5-270.0 NTML270.0

ANCS-270.0 AZMM-270.0

ANC5-270.0 NTML270.0

NeS-270.0 ATML270.

ANa5-270.0 AZMm-270.0

AMes-270.0 NONW-270.0

AMes-273.4 A2M 273.4
AMBS= 90.0 AZML 00.0

Nic5 270.0 ATM 270.0

ANCS-270.0 AZM 270.0
EVENT

EVENT

Evant

EVaNT

EVIT

EVert

EVENT

EVENT

EVENT

EVENT

EVENT

EVENT

EVENT

EVENT

EVENT

EVENT

EVEN

EVENT

EVENT

GVET

EVENT

EVENT

GVant

EVENT

EVENT

EVENT

EVENT

EVENT

EVENT

EVENT

EVENT

EVENT

EVENT

EVENT

EVENT

EVENT

EVENT

EVENT

EVENT

EVENT

EVENT

EVENT

EVENT

EVENT

EVENT

EVaNT

EVENT

EVENT

EVENT

EVENT

EVENT

EVENT

EVENT

EVaN

EVENT

EVaNT

EVENT

Event

Event

EVENT

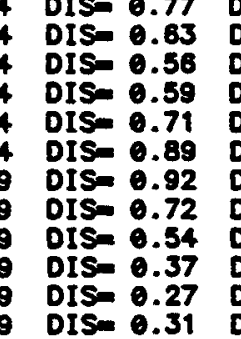

9 DIS= 0.45

DIS= 0.63

9 DIS- 0.82

14 DIS- 0.98

14 DIS 0.80

14 DIS- 0.64

14 DIS= 0.58

14 DIS= 0.5

14 DIS= 0.69

14 DIS 0.88

13 DIS 0.07

13 DIS- 0.77

13 DIS- 0.59

13 DIS 0.42

13 DIS- 0.31

13 DIS= 0.31

13 DIS 0.42

13 DIS- 0.59

13 DIS- 0.77

13 DIS- 0.87

12 DIS- 0.86

12 DIS e. 0.

12 DIS- 0.58

12 DIS- 0.58

12 DIS 0.64

12 DIS- 0.80

12 DIS $=0.99$

DIS- 0.82

7 DISm 0.63

7 DIS- 0.45

7 DIS- 0.31

7 DIS= 0.27

7 DIS= 0.37

7 DIS 0.54

7 OIS $=0.72$

DIS- 0.92

2 DIS 0.89

2 DIS- 0.71

DIS- 0.59

2 DIS- 0.58

2 DIS= 0.63

DIS 0.77

2 DIS- 0.96

3 DIS- 0.87

3 DIS 0.68

3 DIS- 0.30

3 DIS- 0.35

3 DIS- 0.29 
FING:. 11, 65 ELENENTS RADIUS- 2.29

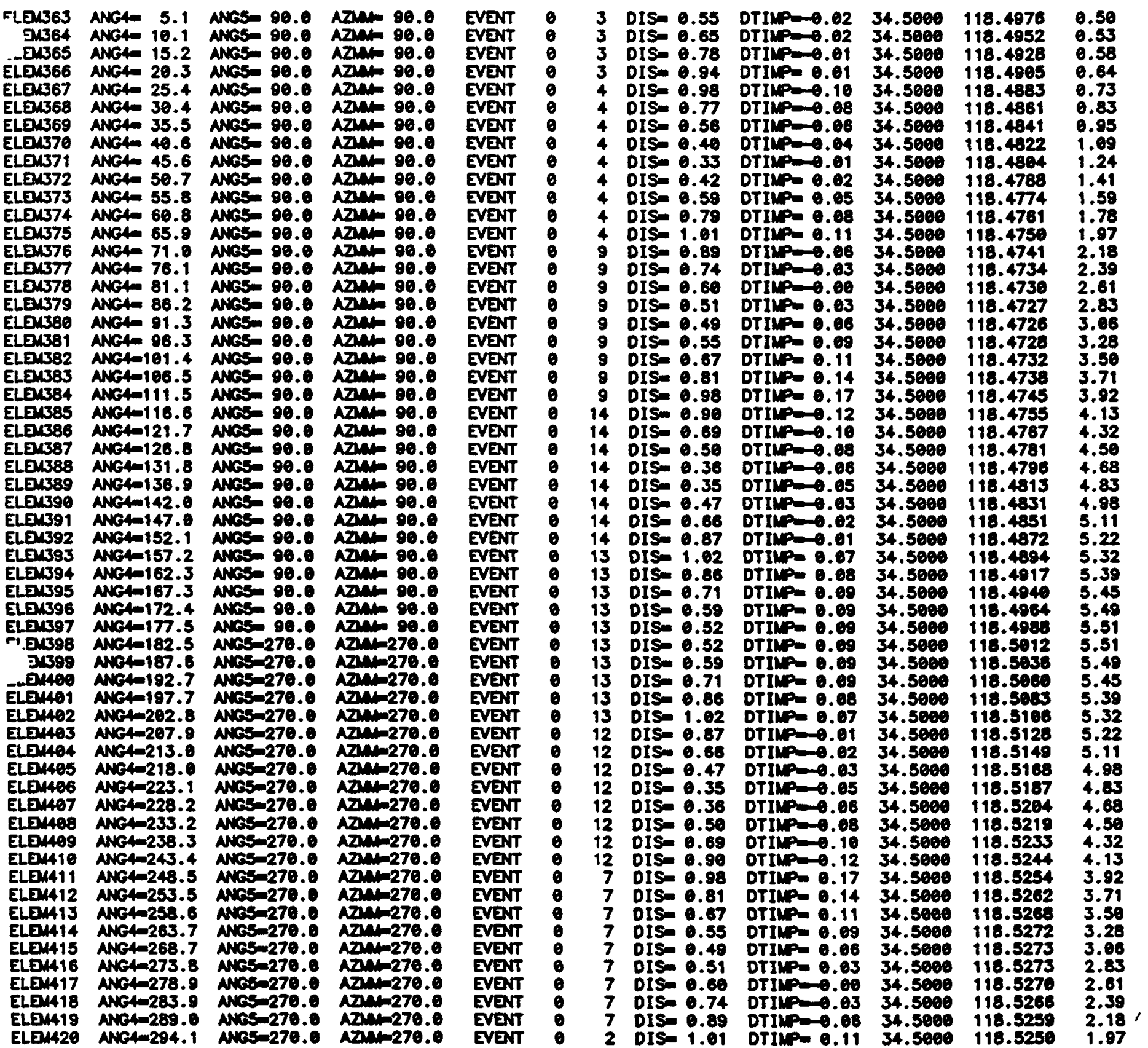


ELEM421 ANG4-299.2 ANGS-270.0 MZML-270.0 ELEM22 ANG4-304.2 ANCE 270.0 MZML270.0 FLEM423 ANG4-309.3 ANG5-270.0 AZMm-270.0 31424 ANG4-314.4 ANOS-270.0 AZML270.0 -01425 ANG4-318.4 ANCS-270.0 ATML270.0 ELEN426 NNG4-324.5 ANCS-270.0 AZMN-270.0 ELEM427 ANG4-329.6 ANGS-270.0 AZMK=270.0 ELE1428 NGG4-334.6 NKC5 $=270.0$ ATML270.0 ELEN429 ANG4-339.7 NNG5=270.0 ATNL270.0 ELEN430 ANG4-344.8 NKCS=270.0 AZML=270.0 ELDH431 ANG4-349.9 ANES-270.0 AZML270.0 ELEN432 ANG4-354.9 ANE5-270.0 AZML270.0 ELEM433 ANG4-360.0 ANCS-273.4 AZML273.4 RING: 12, 71 ELEMENTS RADIUS- 2.51

ELEN434 ANG4 4.7 NNG5 90.0 Nanm 90.0 ELEN435 NNG4 9.4 NKE5 90.0 AZM 90.0

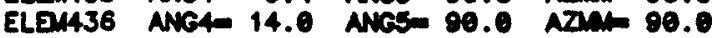
ELEM437 NGC4 18.7 ANCS- 99.0 ATM 90.0 ELEH438 ANG4 23.4 NWES 90.0 AZMK 00.0 ELEN439 AKA 28.1 NKS 90.0 ADMW 90.0 ELgN440 ANG4 32.7 NGeS 90.0 ATMm 90.0 ELEN441 NC4 37.4 NHES 90.0 NTM 90.0 ELaN442 ANG4 42.1 NNES 90.0 AZML 90.0

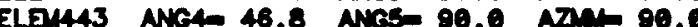

ELEN44 NHC4- 51.4 NWE 90.0 ATML 90.0

ELEN445 ANC4 58.1 ANCS 90.0 AZMm 90.0

ELON446 ANC4 60.8 NMS 90.0 ATM 90.0

ELDU447 ANG4 65.5 ANG5 90.0 AZMN 90.0

ELEN48 ANG4 70.1 ANGS 90.0 AZNK 90.0

ELEM49 NKC4 74.8 NKG5 80.8 ATMN 90.8

ELEMS0 NNG4- 79.5 NNGS- 90.0 ATML 90.0

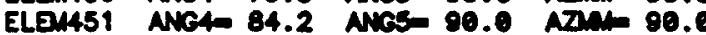

ELEM452 ANG4- 88.8 ANG5 90.0 AZML 90.0

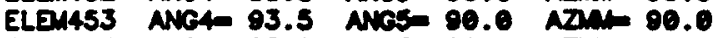

ELEN454 NKC4 88.2 NNCS- 90.0 NZML 00.0

ELEM455 ANG4a102.8 ANES 90.0 NDM 90.0

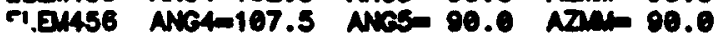

9457 ANG4-112.2 ANes 00.0 ATM 90.0

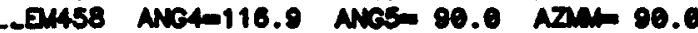

ELEH49 NA4-121.6 NWS- 96.0 NZM 00.0

ELDM60 ANC4m126.2 ANes 90.0 ATM 90.0

ELEM461 NKG4130.9 ANES 90.0 AZMM 90.0

ELEN462 ANC4-135.6 NNG5 90.0 AZM 90.0

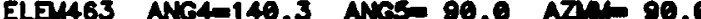

ELEM464 NWG-144.0 ANBS- 90.0 AZM 90.0

ELEN465 ANG4-149.6 NNGS- 90.0 AZML 90.0

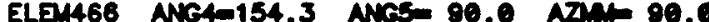

ELEM467 ANG4=159.0 ANG5= 90.0 AZM 90.0

ELDN468 ANG4-163.6 ANG5 90.0 AZML 90.0

ELE1469 ANG4-168.3 MNG5 80.0 NZML 90.0

ELEN470 NNG4173.0 ANGS- 90.0 AZML 90.0

ELEN471 ANG4-177.7 ANGS- 00.0 NZM 00.0

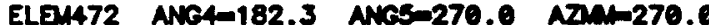

ELEN473 ANG4-187.0 ANGS-270.0 AZNM-270.0

ELEM474 ANG4-191.7 ANGS-270.0 AZML270.0

ELEM475 NG4-196.4 NNCS-270.0 NTML270.0

ELEN476 ANG4-201.0 ANGS-270.0 AZML-270.0

ELEN477 NMG4-205.7 NKGS-270.0 ADN-270.9

ELEH478 ANG4=210.4 ANGS-270.0 AZMW-270.0

$\begin{array}{ll}\text { EVENT } & 0 \\ \text { EVENT } & 0 \\ \text { EVENT } & 0 \\ \text { EVENT } & 0 \\ \text { EVENT } & 0 \\ \text { EVENT } & 0 \\ \text { EVENT } & 0 \\ \text { EVENT } & 0 \\ \text { EVENT } & 0 \\ \text { EVENT } & 0 \\ \text { EVENT } & 0 \\ \text { EVaNT } & 0 \\ \text { EVENT } & 0\end{array}$

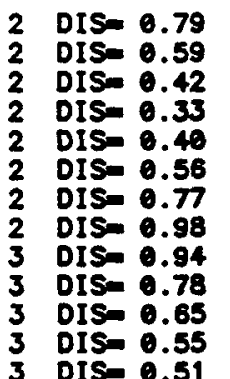

DTIMPa 0.08
DTIMP 0.05

DTIMP 0.02

OTINP -0.01

DTIMP -0.04

DTIMP -0.06

DTIMP -0.08

DTINP=- 0.10

DTINP= 0.01

DTIMP-0.01

DTIMP -0.02

DTIN $=0.02$

DTIMP-0.02

34.5000
34.5000
34.5000
34.5000
34.5000
34.5000
34.5000
34.5000
34.5000
34.5000
34.5000
34.5000
34.5000

118.5239

118.5226

118.5212

118.5195

118.5178

118.5159

118.5138

118.5117

118.5095

118.5072

118.5048

118.5024

118.5000

EVENT

EVENT

EVENT

EVENT

EVENT

EVENT

EVENT

EvanT

Event

EVENT

EVENT

EVENT

EVENT

EVENT

EVErT

EverT

EVENT

EVErt

EVENT

EVENT

Evart

EVENT

EVant

EVan

EVET

Evert

EVanT

EVENT

EVENT

EVENT

EVENT

EVENT

EVENT

EVENT

EVENT

EVant

EVENT

EVant

EVENT

EVENT

EVENT
3 DIS- 0.76

3 DIS= 0.83

3 DIS- 0.93

3 DIS- 1.08

4 DISo 1.08

4 015= 0.84

4 DIS- 0.82

DIS 0.40

4 DIS- 0.18

4 DIS 0.13

4 DIS- 0.32

4 DIS- 0.54

DIS= 0.76

OIS- 0.98

9 DIS- 1.08

DIS- 0.95

9 DIS= 0.84

DIS- 0.76

DIS= 0.72

DIS- 0.73

DIS 0.79

DIS= 0.89

DIS= 1.01

4 DIS- 1.09

14 DIS- 0.87

14 DIS= 0.85

14 DIS- 0.43

14 DIS 0.21

14 DIS- 0.11

14 DIS= 0.29

14 DIS= 0.51

14 DIS- 0.73

14 DIS- 0.95

13 DISn 1.13

13 DIS- 0.99

13 DIS- 0.88

13 DIS 0.79

13 DIs 0.74

13 DIS- 0.74

13 DIS 0.79

13 DIS- 0.88

13 DIS 0.98

13 DIS- 1.13

12 DIS 0.95

12 DIS= 0.73
DTIMP-0.03 34.5000

DTIMP-0.01

DTIMP -0.08

DTIMP -0.07

DTINP=0.05

DTIMP -0.02

DTINo 0.00

DTIN= 0.03

DTIMP 0.08

DTIMP 0.09

OTINPa 0.13

DTIMPm- 0.05

DTINP=0.02

DTIMP 0.01

DTIMP= 0.04

DTIM 0.07

DTIMP 0.10

OTIMP 0.12

DTIP 0.15

DTINP 0.18

DTIMP-0.11

DTIMP -0.08

DTINP -0.07

DTINP=0.05

DTIN $=0.03$

DTINP 0.01

DTIMP 0.00

DTIMP $=0.01$

DTIMP 0.03

DTIM 0.04

DTIMP 0.12

DTIMP 0.12

OTIMP 0.13

DTIMP 0.13

DTIMP $=0.13$

DTIMP= 0.13

DTIMP 0.13

DTIMP 0.13

DTINP= 0.12

DTIMP= 0.12

DTIMPm 0.04

DTINP= 0.03
DTINP-0.02

DTIM-0.00
$5000 \quad 118.4951$

$34.5000 \quad 118.4928$

$34.5000 \quad 118.4904$

$34.5000 \quad 118.4882$

34.5000118 .4860

$34.5000 \quad 118.4839$

$34.5890 \quad 118.4818$

$34.5600 \quad 118.4800$

$34.5900 \quad 118.4783$

$34.5600 \quad 110.4767$

$34.5000 \quad 118.4753$

34.5000

34.5000

34.5000

34.5000

34.5000

34.5000

34.5000

34.5000

34.5000

34.5060

34.5000

34.5000

34.5000

34.5000

34.5600

34.5000

34.5000

34.5000

34.5800

34.5000

34.5000

34.5000

34.5000

34.5000

34.5000

34.5000

34.5000

34.5000

34.5000

34.5000

34.5000

34.5000

34.5000

118.4746

118.4729

118.4720

118.4712

118.4707

118.4704

118.4702

118.4702

118.4705

118.4709

118.4718

118.4724

118.4734

118.4746

118.4760

118.4775

118.4791

118.4800

118.4828

118.4840

118.4871

118.4893

118.4918

118.4940 


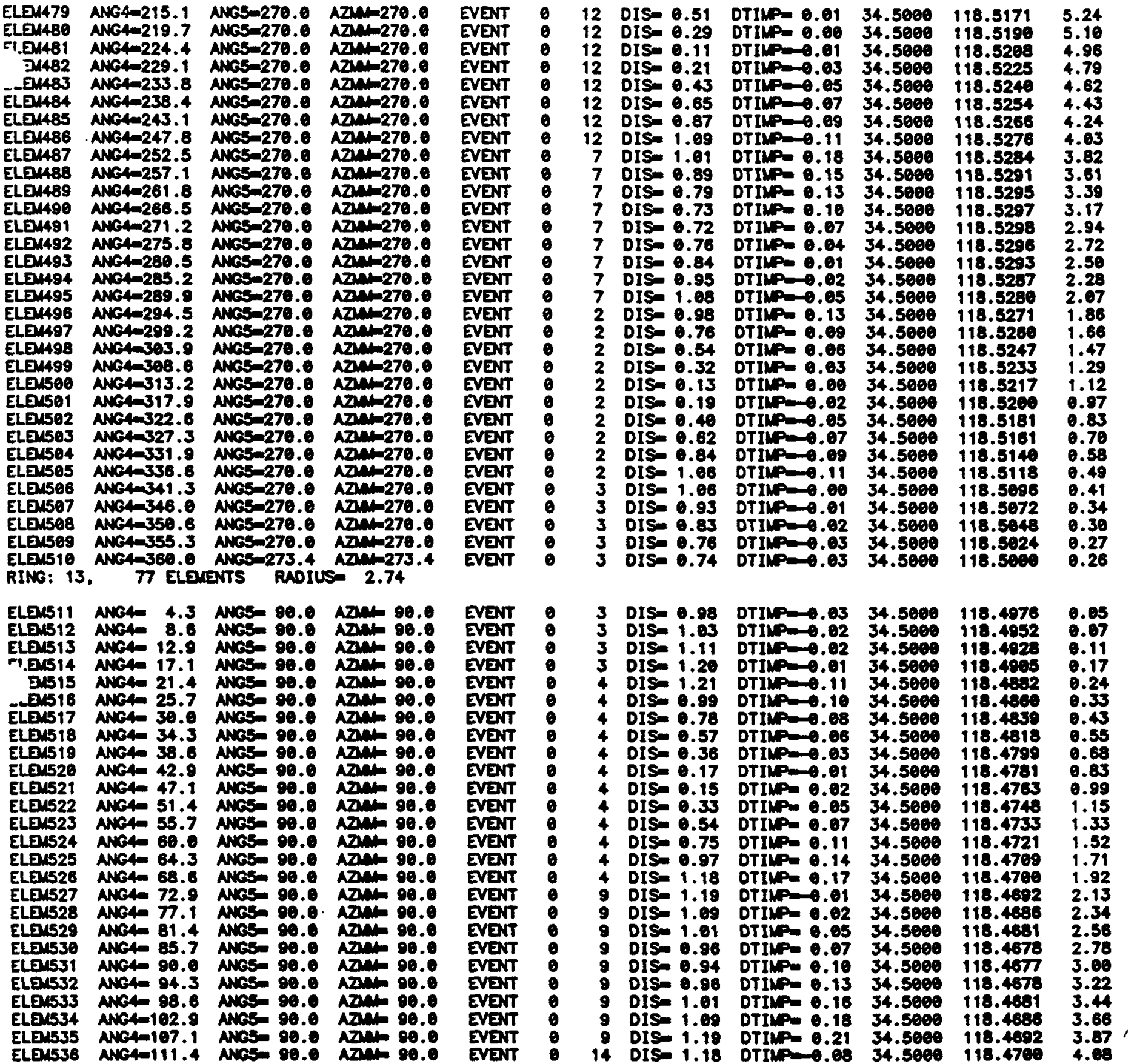


ELEN537 ANG4-115.7 NKOS 90.0 AZMK 90.0 ELEL538 ANG4-120.0 ANG5 90.0 A7ML 90.0 ELEL539 ANG4-124.3 ANGS 90.0 AZMM 90.0 EN540 ANG4128.6 ANCS= 90.0 AZML 90.0 04541 ANG4-132.9 ANC5= 90.0 AZML 90.0 ELEN542 ANG4-137.1 ANGS= 90.0 AZML 90.0 ELEK543 NG4=141.4 ANGS- 90.0 AZML 90.0 ELEN544 ANG4-145.7 NGS5 90.0 ATML 90.0 ELEN545 ANG4-150.0 ANC5- 90.0 AZMN 90.0 ELEM546 ANG4-154.3 ANGS- 90.0 AZML 90.0 ELEU547 ANG4-158.6 NCSK 90.0 AZML 90.0 ELEU548 ANG4-162.9 ANC5-90.0 AZML 90.0 ELEU549 ANG4-167.1 ANG5- 90.0 AZML 90.0 ELEU550 ANG4-171.4 NCS- 90.0 AZML 90.0 ELEU551 ANC4-175.7 ANC5- 90.0 AZMW 90.0

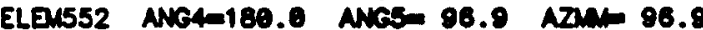
ELDU553 ANG4-184.3 NGSm270.0 AZM-270.0 ELEN554 ANG4-188.8 NNES-276.0 AZN-270.0 ELEL555 ANG4-192.9 NCS5=270.0 ATML270.0

ELEN556 ANC4=197.1 ANCS-270.0 AZML270.0

ELEU557 ANC4-201.4 ANES-270.0 AZMK-270.0

ELEU558 ANG4-205.7 ANOS-270.0 ATML270.0

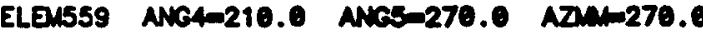

ELEN560 ANG4-214.3 ANCS-270.0 AZNW270.0

ELDL561 ANG4-218.8 ANes-270.0 ATMس270.0

ELDU562 ANG4-222.9 ANCS=270.0 ATN-270.0

ELEM563 ANG4-227.1 ANG5-270.0 AZM-270.0

ELEN564 ANG4-231.4 NGS-270.0 ATML270.0

ELEK565 ANG4=235.7 NGGS-270.0 AZMW-270.0

ELEN566 ANG4-240.0 NMGS-270.0 ATML270.0

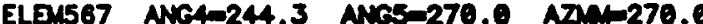

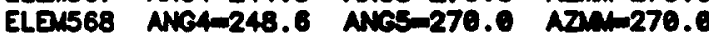

ELEV569 ANG4-252.9 ANCS-270.0 ATML270.0

ELEU570 ANG4-257.1 NMGS-270.0 AZM-270.0

ELEN571 NKG4-281.4 NMGS-270.0 AZN-270.0

ELOK572 ANG4-265.7 ANCS-270.0 ATML270.0

ELDU573 NMS4-270.0 MUE-270.0 AZMK-270.0

CLEN574 NMC4-274.3 ANGS-270.0 ANML270.0

Eu576 ANC4-282.0 NCS-270.0 A7Nm-270.0

ELE1577 NMC4-287.1 NKGS-270.0 AZNM-270.0

ELEL578 ANC4-291.4 ANES-270.0 ATNM270.0

ELEN579 NMG4-295.7 NNGS-270.0 NZNW270.0

ELEU580 ANG4-300.0 NKS-270.0 ATM-270.0

ELEL581 ANG4-304.3 NKOS-270.0 ATML270.6

ELEMS82 ANC4-308.6 NKS5-270.0 AZMW-270.0

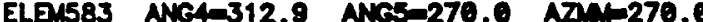

ELEU584 ANC4-317.1 ANCS-270.0 AZMm270.0

ELEN585 ANG4-321.4 ANGS=270.0 AZMM-270.0

ELEM586 ANG4-325.7 NNES-270.0 AZM-270.0

ELEL587 ANG4-330.0 NKC5-270.0 AZM 270.0

ELEM588 ANC4-334.3 NGS-278.0 AZM-270.0

ELD589 ANG4338.6 ANGS-270.0 AZMm270.0

ELEN590 NVE-342.9 NNES-270.0 AZMW-270.0

ELDN591 ANG4-347.1 NNG5-270.0 AZNM-270.0

ELDL592 ANG4-351.4 ANGS-270.0 ATMW270.0

ELEU593 ANC4-355.7 ANCS-270.0 AZMW-270.0

ELEMS94 ANG4-360.0 NWSS-273.4 AZML-273.4

RIMG: 14,84 ELEMENTS RADIUS- 2.96

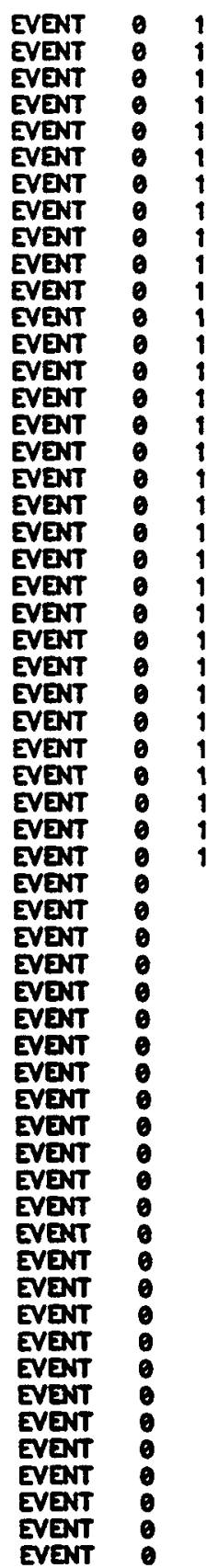

14 DIS- 0.97

14 015 0.75

14 DIS- 0.54

4 DIS= 0.33

4 DIS= 0.15

14 DIS- 0.17

14 DIS- 0.38

4 DIS= 0.57

4 DIS= 0.78

4 DIS= 0.99

14 DISE 1.21

13 DIS- 1.20

DIS= 1.11

DISn 1.03

DIS- 0.98

DIS= 0.96

DIS 0.98

DIS= 1.03

DIS- 1.18

DIS- 1.20

DIS= 1.21

DIS= 0.99

DIS= 0.78

DIS $=0.57$

DIS- 0.38

DIS- 0.17

DIS- 0.15

DIS- 0.33

DIS- 0.54

DIS $=0.75$

DIS= 0.97

DIS- 1.18

DIS- 1.19

DIS- 1.09

DIS- 0.08

DIS- 0.04

DIS= 0.08

DIS- 1.01

DIS- 1.09

DIS 1.19

DISE 1.18

DIS= 0.97

DIS= 0.75

DIS= 0.54

DIs- 0.33

DIS 0.15

DIS- 0.17

DIS- 0.57

DIS- 0.78

DIS 0.99

DIS- 1.21

DIS- 1.20

DIS 1.11

DIS- 1.03

DIS 0.98

DIS 0.96

DTIMP -0.06

DTIMP -0.02

DTIMP- 0.00

DTIN= 0.01

DTIMP $=0.03$

OTIMP 0.04

DTIM= 0.08

DTINP 0.07

DTINP 0.08

DTIN= 0.69

DTINP 0.16

DTIMP 0.17

0.17

DTIMP 0.18

DTIN $=0.18$

TIMP $=0.18$

DTIMP 0.17

DTIMP 0.17

DTINa 0.16

DTINP 0.09

DTIMP 0.08

DTIMP $=0.07$

DTINP= 0.06

DTIM 0.04

DTIMPm 0.03

DTIMP 0.01

DTIM $=0.00$

DTIM -0.02

OTINP-0.04

DTIMP-0.08

DTIM -0.08

DTIMP 0.21

DTIMP $=0.18$

DTIMP 0.18

DTIMPe 0.13

DTINP 0.10

DTIMP= 0.07

DTIVP 0.05

DTIMP $=0.02$

OTIM $=0.01$

DTIMP 0.17

DTINP 0.14

0.11

DTINP 0.07

DTIMP 0.05

DTIN 0.02

DTIMP-0.01

DTINP- 0.03

OTIMPa-0.08

DTIMP -0.08

DTIMP -0.10

DTIMP- 0.11

DTIMP -0.01

DTIMPD-0.02

DTIMP=0.02

DTIMP -0.03

DTIMP -0.03

34.5600

118.4721

118.4733

118.4748

118.4763

118.4781

118.4798

118.4818

118.4839

118.4860

118.4882

118.4905

118.4928

118.4952

118.4976

118.5600

118.5024

118.5848

118.5972

118.5095

118.5118

118.5140

118.5161

118.5182

118.5201

118.5210

118.5236

118.5252

118.5286

118.5270

118.5291

118.5300

118.5308

118.5314

118.5318

118.5322

118.5322

118.5322

118.5318

118.5314

118.5380

118.5390

118.5291

118.5278

118.5288

118.5252

118.5238

118.5210

118.5201

118.5182

118.5161

118.5140

118.5118

118.5095

118.5072

118.5048

118.5024

118.5600
4.29

4.48

4.67

4.85

5.61

5.17

5.32

5.45

5.57

5.67

5.76

5.83

5.89

5.83

5.95

3.86

5.95

5.89

5.83

5.76

5.57

5.45

5.32

5.17

4.85

4.67

4.48

4.29

4.08

3.87

3.86

3.44

3.22

3.00

2.78

2.56

2.34

2.13

1.92

1.71

1.52

1.33

1.15

0.99

0.83

0.88

0.43

0.33

.24

0.17

0.11

0.05

0.04 

ELEL596 NMG4 24.0 NMC5 90.0 AZM 90.0 3 J598 ANG4 32.0 NGG5- 90.0 AZML 90.0

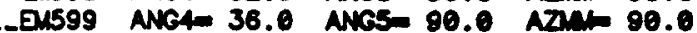
ELEN600 ANG4 40.0 ANC5- 90.0 AZML 90.0 ELEN601 ANG4 44.0 ANG5 90.0 ADML 90.0 ELEN602 ANG4 48.0 NKC5 90.0 ATM 90.0 ELDMGO3 ANC4 52.0 NMCS- 90.0 AZML 90.0 ELEN604 ANG4 56.0 ANC5 90.0 AZN 90.0 ELEN695 ANG4 60.9 ANCS 90.0 ATMm 09.0 ELEN606 ANG4 64.0 ANCS 90.0 ATM 90.0 ELEN607 ANG4 68.0 ANG5- 90.0 AZM 90.0 ELEV608 ANG4 72.0 ANCS- 90.0 A7mm 00.0 ELEN609 ANG4 76.0 ANGS- 90.0 AZML 90.0 ELEN610 ANG4 80.0 NWOS 90.0 ANML 80.0

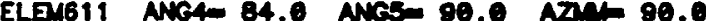
ELEM612 ANG4 88.0 NMGS 90.0 AZNh 80.0 ELENG13 NMG4 92.0 NMGS 90.0 ANML 90.0 ELEVB14 AMC4 98.0 AMC6 90.0 ATML 90.0

ELEM615 NMG4=100.0 NMGS- 90.0 AZML 80.0

ELEM618 ANG4-104.0 NGS- 90.0 ATMW 90.0

ELEV617 ANG4-108. ANGS- 09.9 ATM 90.0

ELEL618 MG4=112.0 NKCS- 90.0 AZML 90.0

ELEMB19 ANG4-116.0 ANGS 90.0 ATMm 90.0

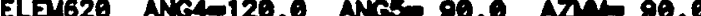

ELEM621 ANG4-124.0 MNES 90.0 AZML 90.0

ELEN622 ANG4-128.0 NNC5- 90.0 AZML 90.0

ELEV623 ANG4-132. ANC5 00.0 ATML 90.0

ELEN624 ANG4-136.0 ANC6- 90.0 AZ1M 90.0

ELEM625 ANG4-140.0 ANOS- 90.0 ATML 90.0

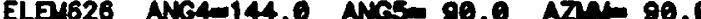

ELEN627 ANG4148.0 ANBS 90.0 AZM 90.0

ELEN628 AKG4-152.0 AMES- 90.0 AZML 90.0

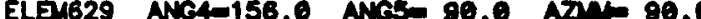

ELEN630 ANC4-160.0 NMS5 90.0 ATML 90.0

ELEN831 NWG 200.0 NNG5-270.0 ATM-270.0

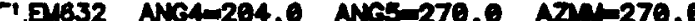

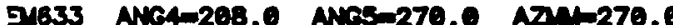

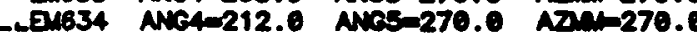

ELEM635 ANC4-216. ANCS-270. ATML270.0

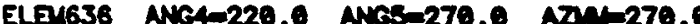

ELEN637 ANC4-224.0 ANCS-270.0 AZNM-270.0

LLE1638 ANG4-228.6 ANeS-270.0 ATMm270.0

ELEN639 ANG4-232.0 ANGS-270.0 ATM-270.0

ELEN640 ANG4=236.0 ANGS-270.0 ATML270.0

ELEM641 AKG4-240.0 NOS-270.0 NTMU270.0

ELEM642 NGG-244.0 NOS-270.0 ATML270.0

ELEN643 NNG4-248.0 NNCS-270.0 AZML270.0

ELEN644 NGG4252.0 AMG5 270.0 ATML270.0

ELEN645 MG4-256.0 ANG5=270.0 ATML270.0

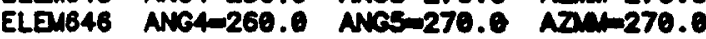

ELEN647 NG4 264.0 NGS-270.0 ATML270.0

ELEM648 MG4-268. ANCS-270. ATM-270.0

ELEM649 ANG4-272.0 ANCS-270.0 NTW-270.0

ELDK650 ANG4-276.0 ANC5-270.0 ATMm270.0

ELEN651 ANG4-280.0 NCS-270.0 ATML270.0

ELEN652 ANG4-284.0 ANC5-270.0 NZN-270.0

ELE 653 ANG4-288.0 ANC5 270.0 ATM-270.0

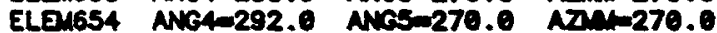

\begin{tabular}{|c|}
\hline 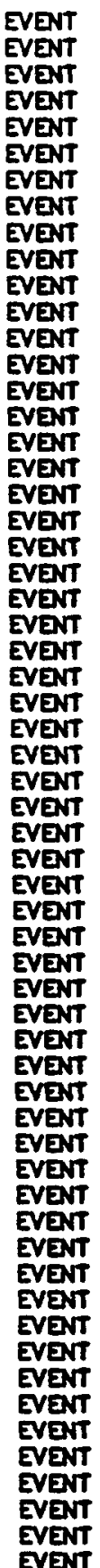 \\
\hline
\end{tabular}$$
4
$$$$
4 \text { OIS- } 1.16
$$$$
4 \text { DIS- } 0.78
$$$$
4 \text { DIS= } 0.60
$$$$
4 \text { DIS } 9 .
$$$$
4 \text { DIS- } 0.35
$$$$
4 \text { DIS= } 9.37
$$$$
\text { DISo } 0.48
$$$$
4 \text { DIS- } 0.86
$$$$
4 \text { DIS= } 0.84
$$$$
\text { DIS= } 1.04
$$$$
4 \text { DIS= } 1.24
$$$$
\text { DIS- } 1.38
$$$$
10 \text { DIS= } 1.20
$$$$
10 \text { DIS- } 1.03
$$$$
10 \text { DIS- } 0.91
$$$$
16 \text { DIS } 0.84
$$$$
10 \text { OIS= } 0.84
$$$$
10 \text { DIS= } 0.01
$$$$
18
$$$$
10 \text { DISw } 1.20
$$$$
\text { DIS- } 1.38
$$$$
14 \text { DIS- } 1.24
$$$$
14 \text { DIS= } 1.04
$$$$
14 \text { DIS- } 0.84
$$$$
14 \text { DIS- } 0.68
$$$$
14 \text { DIS= } 0.49
$$$$
14 \text { DIS= } 0.37
$$$$
14 \text { DIS- } 0.35
$$$$
14 \text { DIS- } 0.44
$$$$
14 \text { DIS- } 0.60
$$$$
14 \text { DIS= } 0.78
$$$$
14 \text { DIS= } 0.97
$$$$
14 \text { DIS- } 1.16
$$$$
14 \text { DIS- } 1.36
$$$$
12 \text { ols- } 1.38
$$$$
12 \text { DIS- } 1.16
$$$$
12 \text { DIS- } 0.97
$$$$
12 \text { DIS } 9.78
$$$$
2 \text { DIS= } 0.60
$$$$
12 \text { DIS- } 0.44
$$$$
12 \text { DIS- } 0.35
$$$$
\text { DIS- } 0.37
$$$$
\text { DIS } 0.49
$$$$
\text { DIS= } 0.66
$$$$
\text { DIS= } 0.84
$$

DIS= 1.04

DIS= 1.24

DIS- 1.38

DIS- 1.20

DIS- 1.03

DIS- 0.81

DIS= 0.84

DIS= 0.84

DIS= 0.81

DISo 1.03

DIS- 1.20

DIS- 1.38

2 DIS- 1.24
DTIMP-0.11

DTINP=-0.10 DTIMP -0.08

DTIMP -0.08

DTINP=0.04

TIMP=-0.02

DTINP= 0.01

DTINP 9.04

TINP= 0.06

DTIMP 0.09

DTIM $=0.12$

OTIMP 0.18

DTINP 0.19

DTINP 0.00

DTIMP-0.22

DTINP -0.18

DTIMP-0.18

DTIMP -0.13

DTIMP -0.11

DTIMP-0.08

DTIMP-0.05

DTIMP- 0.03

DTINP 0.25

DTIMP $=0.05$

DTIMP-0.03

DTIMP-6.01

DTIMP 0.01

DTIMP 0.03

DTINP= 0.05

DTINP= 0.08

DTIMP $=0.08$

DTIMP 0.09

DTIMPs 0.10

DTIMP 0.11

DTIMP 0.12

DTIMP 0.13

DTIMP 0.13

DTIMP 0.12

DTINP 0.11

DTINP 0.10

DTINP 0.08

DTINP= 0.08

DTIMP 0.08

DTIMP 0.05

DTIMP= 0.03

DTIMP $=0.01$

OTINP=0.01

OTIN $=0.03$

DTIMP-0.05

DTINP 0.25

DTIMP-0.03

DTINP -0.05

DTIMP-0.08

OTIN $=0.11$

DTINP-0.13

DTINP-0.16

DTIMP= 0.19

DTIMP 0.22

DTIMP 0.00

DTIMP 0.19
34.5000

34.5000

34.5000

34.5000

34.5000

34.5000

34.5600

34.5000

34.5600

34.5000

34.5000

34.5600

34.5000

34.5000

34.5000

34.5000

34.5000

34.5000

34.5000

34.5080

34.5000

34.5060

34.5000

34.5000

34.5000

34.5000

34.5000

34.5000

34.5000

34.5000

34.5000

34.5000

34.5000

34.5000

34.5000

34.5000

34.5000

34.5600

34.5600

34.5000

34.5600

34.5000

34.5800

34.5000

34.5000

34.5000

34.5000

34.5000

34.5000

34.5000

34.5000

34.5000

34.5000

34.5000

34.5000

34.5600

34.5000

34.5000

34.5800

118.4881

118.4859

118.4837

118.4816

118.4796

118.4777

118.4759

118.4742

118.4726

118.4712

118.4690

118.4688

118.4678

118.4670

118.4683

118.4658

118.4655

118.4653

118.4653

118.4655

118.4658

118.4663

18.4670

118.4678

118.4688

118.4690

118.4712

118.4726

118.4742

118.4750

118.4777

118.4796

118.4818

118.4837

118.4859

118.4881

198.3119

198.5141

118.5163

118.3184

118.5204

118.5223 


\begin{tabular}{|c|c|c|c|c|c|c|c|c|c|c|c|}
\hline $\begin{array}{r}\text { ELEM655 } \\
\text { ELEM656 } \\
\text { FLEM657 } \\
\text { פM658 } \\
\text { EN659 } \\
\text { ELEM660 } \\
\text { ELEM661 } \\
\text { ELEM662 } \\
\text { ELEM663 } \\
\text { ELEN664 } \\
\text { ELE } 6665 \\
\text { ELEM666 } \\
\text { RING: } 15\end{array}$ & 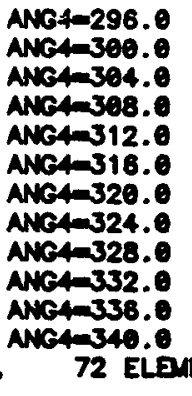 & 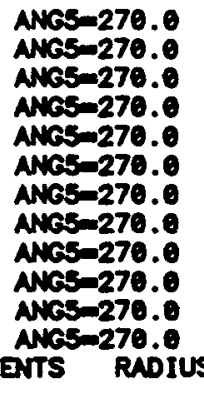 & 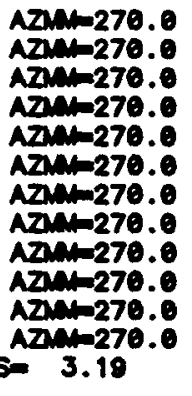 & $\begin{array}{l}\text { EVENT } \\
\text { EVENT } \\
\text { EVENT } \\
\text { EVENT } \\
\text { EVENT } \\
\text { EVENT } \\
\text { EVENT } \\
\text { EVENT } \\
\text { EVENT } \\
\text { EVENT } \\
\text { EVENT } \\
\text { EVENT }\end{array}$ & $\begin{array}{l}0 \\
0 \\
0 \\
0 \\
0 \\
0 \\
0 \\
0 \\
0 \\
0 \\
0 \\
0\end{array}$ & $\begin{array}{l}2 \\
2 \\
2 \\
2 \\
2 \\
2 \\
2 \\
2 \\
2 \\
2 \\
2 \\
2 \\
2 \\
2 \\
2\end{array}$ & 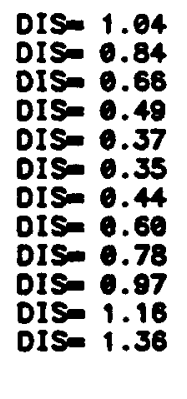 & 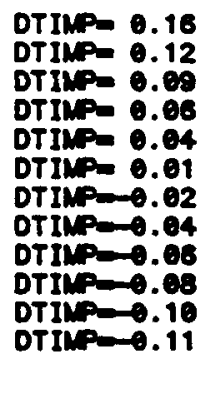 & 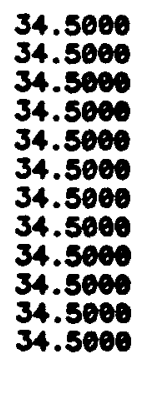 & $\begin{array}{l}118.5312 \\
118.5360 \\
118.5288 \\
118.5273 \\
118.5258 \\
118.5241 \\
118.5223 \\
118.5204 \\
118.5184 \\
118.5163 \\
118.5141 \\
118.5119\end{array}$ & $\begin{array}{l}1.60 \\
1.41 \\
1.22 \\
1.04 \\
0.87 \\
0.71 \\
0.56 \\
0.42 \\
0.30 \\
0.19 \\
0.09 \\
0.01\end{array}$ \\
\hline 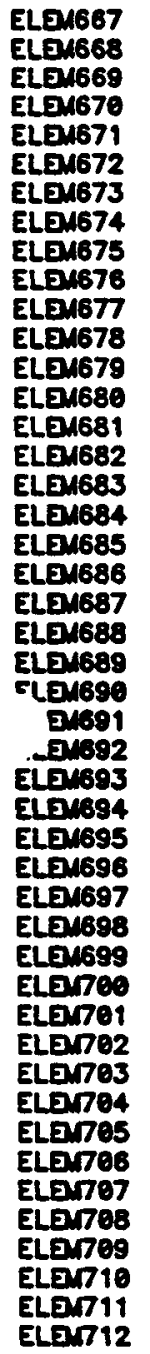 & 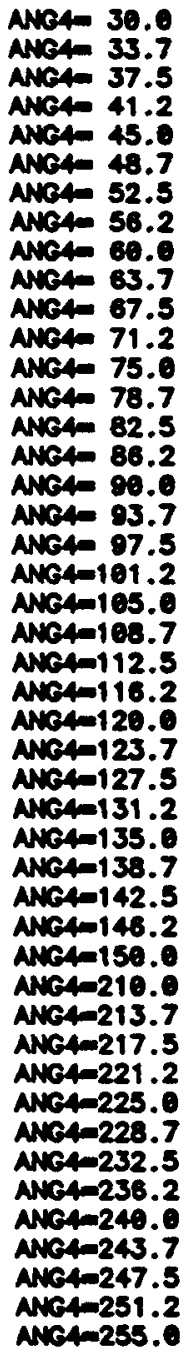 & 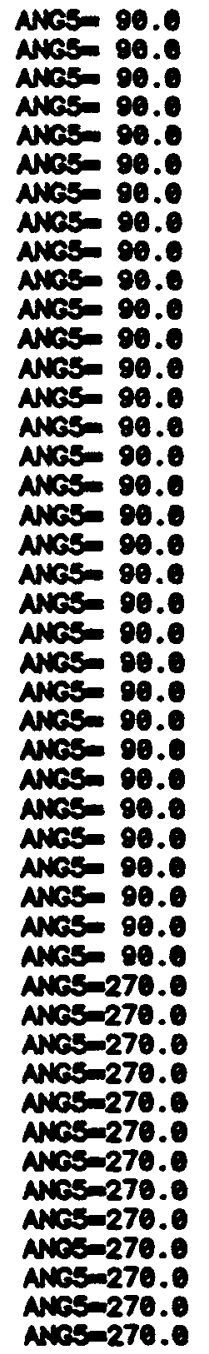 & 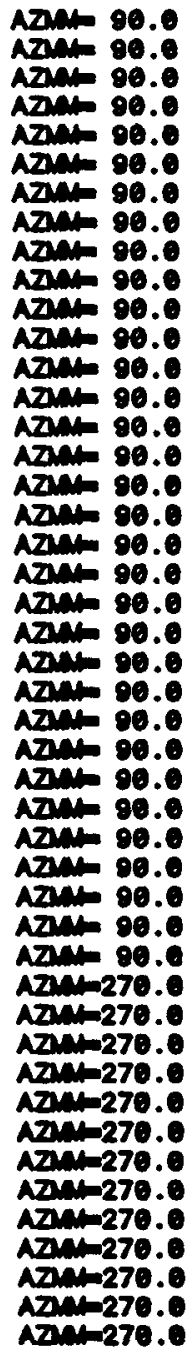 & 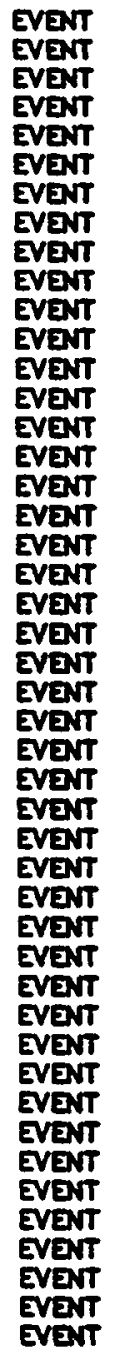 & $\begin{array}{l}0 \\
0 \\
0 \\
0 \\
0 \\
0 \\
0 \\
0 \\
0 \\
0 \\
0 \\
0 \\
0 \\
0 \\
0 \\
0 \\
0 \\
0 \\
0 \\
0 \\
0 \\
0 \\
0 \\
0 \\
0 \\
0 \\
0 \\
0 \\
0 \\
0 \\
0 \\
0 \\
0 \\
0 \\
0 \\
0 \\
0 \\
0 \\
0 \\
0 \\
0 \\
0 \\
0 \\
0 \\
0 \\
0 \\
0\end{array}$ & $\begin{array}{l}4 \\
4 \\
4 \\
4 \\
4 \\
4 \\
4 \\
4 \\
4 \\
5 \\
5 \\
5 \\
10 \\
10 \\
10 \\
10 \\
10 \\
10 \\
10 \\
10 \\
10 \\
15 \\
15 \\
15 \\
14 \\
14 \\
14 \\
14 \\
14 \\
14 \\
14 \\
14 \\
14 \\
12 \\
12 \\
12 \\
12 \\
12 \\
12 \\
12 \\
12 \\
12 \\
11 \\
11 \\
11\end{array}$ & 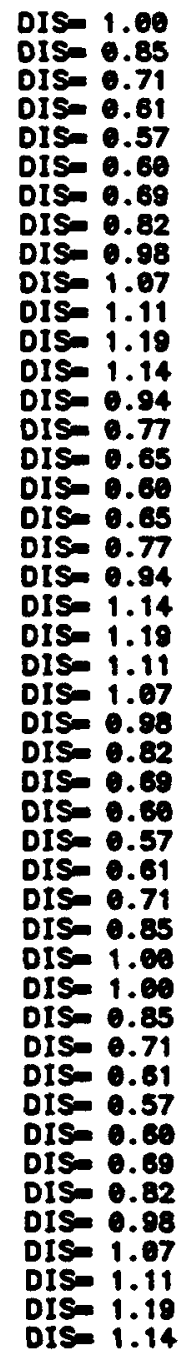 & 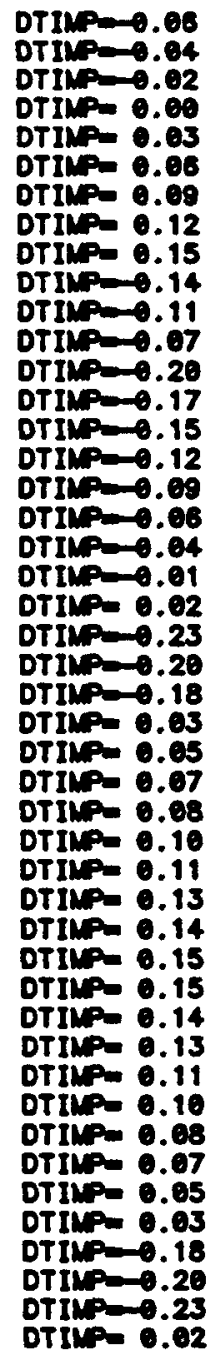 & 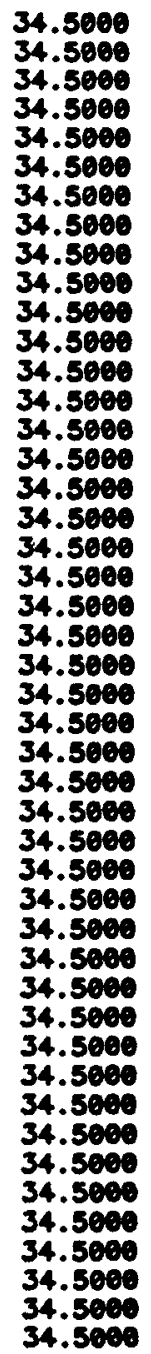 & 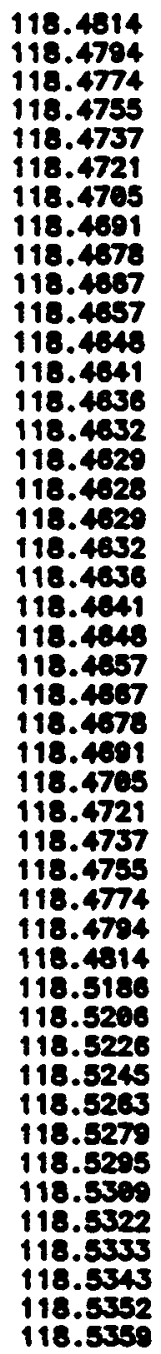 & $\begin{array}{l}0.05 \\
6.16 \\
0.29 \\
0.44 \\
0.59 \\
0.75 \\
0.92 \\
1.16 \\
1.29 \\
1.49 \\
1.69 \\
1.90 \\
2.12 \\
2.33 \\
2.55 \\
2.78 \\
3.80 \\
3.22 \\
3.45 \\
3.67 \\
3.88 \\
4.10 \\
4.31 \\
4.51 \\
4.71 \\
4.90 \\
5.08 \\
5.25 \\
5.41 \\
5.56 \\
5.71 \\
5.84 \\
5.95 \\
5.95 \\
5.84 \\
5.71 \\
5.56 \\
5.41 \\
5.25 \\
5.08 \\
4.90 \\
4.71 \\
4.51 \\
1.51\end{array}$ \\
\hline
\end{tabular}




\begin{tabular}{|c|c|c|c|c|}
\hline 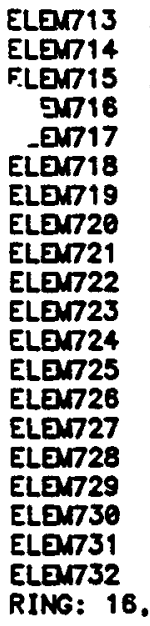 & 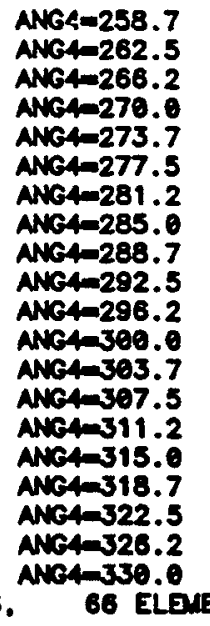 & 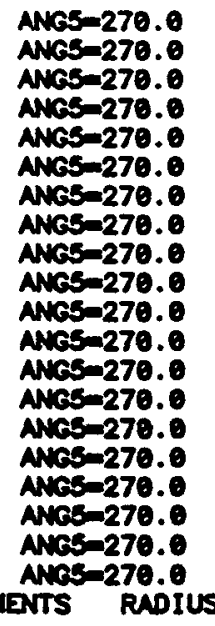 & 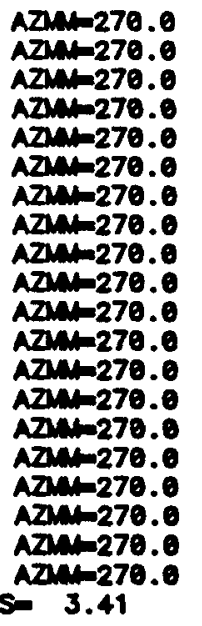 & $\begin{array}{l}\text { EVENT } \\
\text { EVENT } \\
\text { EVENT } \\
\text { EVENT } \\
\text { EVENT } \\
\text { EVENT } \\
\text { EVENT } \\
\text { EVENT } \\
\text { EVENT } \\
\text { EVENT } \\
\text { EVENT } \\
\text { EVENT } \\
\text { EVENT } \\
\text { EVENT } \\
\text { EVENT } \\
\text { EVENT } \\
\text { EVENT } \\
\text { EVENT } \\
\text { EVENT } \\
\text { EVENT }\end{array}$ \\
\hline 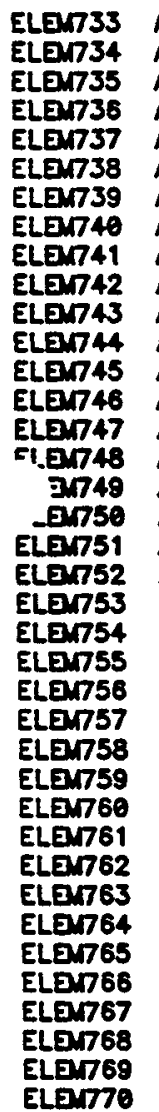 & 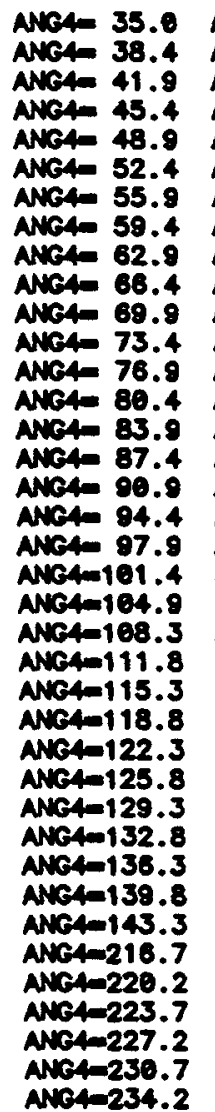 & 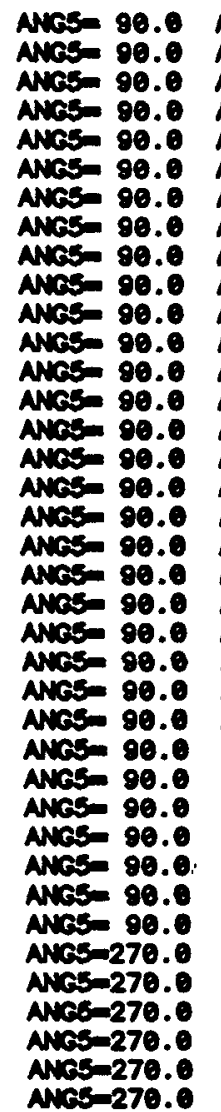 & 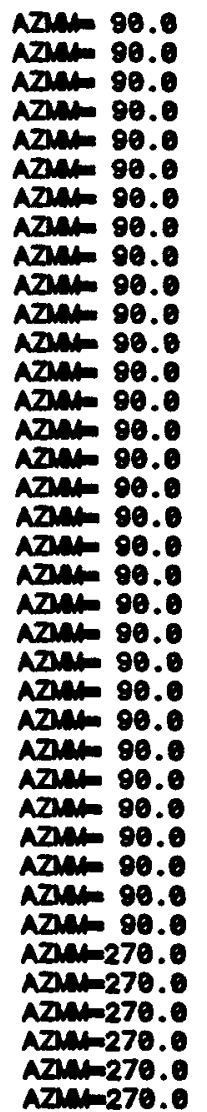 & 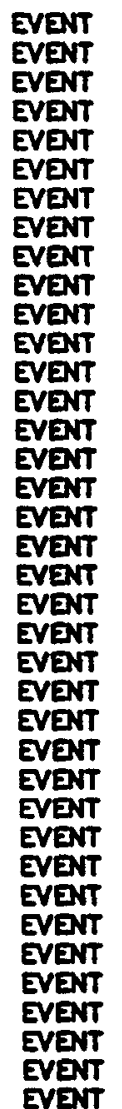 \\
\hline
\end{tabular}

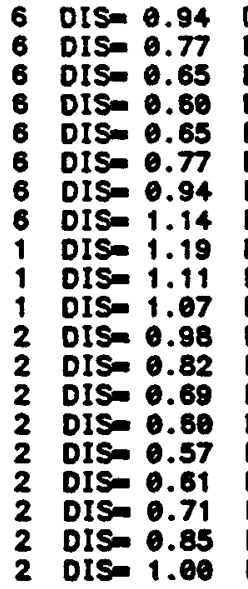

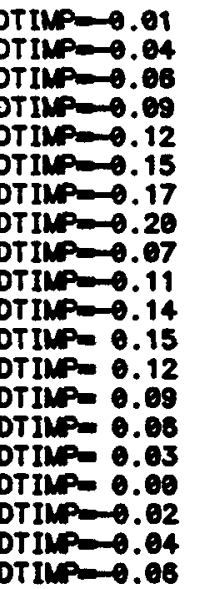
4 DIS- 0.98 4 DIS- 0.88 4 DIS= 0.82 4 DIS- 0.79 4 DIS= 0.82 4 DIS- 0.89 5 DIS- 0.96 5 DIS= 0.90 5 DIS- 0.85 5 DIS- 0.87 5 DIS- 0.96 5 DIS- 1.10 10 DIS- 0.95 10 DISE 0.74 10 DIS= 0.55 10 DIS 0.42 10 DIS- 0.38 10 DIS 0.48 10 DIS= 0.64 10 DIS- 0.84 DIS- 1.08 15 DIS- 1.02 15 DIS- 0.91 15 DIS= 0.85 15 Dis= 0.86 15 Dis- 0.94 14 DISo 0.94 14 DIS= 0.85 14 DIS= 0.80 14 DIS= 0.80 14 DIS= 0.84 14 DISe 0.93 12 DIS 0.93 12 DIS 0.84 12 DIS 0.80 12 DIS 0.80 12 DIS 0.85 12 DIS 0.94
DTIMP -0.02 DTIMP 0.03 DTINP 0.05 DTIMP 0.08 DTIMP 0.11 DTINP -0.18 OTINP=0.15 DTIM $=0.12$ DTIMP $=0.09$ DTIMP -0.03 DTINP -0.16 DTIMP- 0.13 OTIMP -0.10 DTIMP -0.05 DTIMP-0:02 DTIMP 0.00 OTIMP $=0.03$ DTIMP 0.05 DTIMP -0.19 DTINP $=0.17$ DTIMP $=0.15$ DTINP $=0.13$ DTIMP $=0.11$ DTIMP $=0.10$ DTIMP 0.11 DTIMP 0.13 DTIMP 0.15 DTIMP 0.18 DTINP= 0.17 DTIMP 0.17 DTIMP 0.15 DTINP 0.13 DTINP= 0.11 DTINP 0.10 DTIMP 0.00 OTIM -0.08 DTIMP- -0.08 DTINP= 0.18
34.5000 34.5000 34.5000 34.5000 34.5000 34.5000 34.5000 34.5000 34.5600 34.5000 34.5000 34.5600 34.5000 34.5000 34.5600 34.5000 34.5000 34.5000 34.5000

34.5000 34.5000 34.5000 34.5000 34.5000 34.5000 34.5000 34.5000 34.5000 34.5000 34.5600 34.5800 34.5000 34.5000 34.5000 34.5000 34.5000 34.5000 34.5000 34.5000 34.5000 34.5000 34.5000 34.5000 34.5000 34.5000 34.5000 34.5000 34.5000 34.5000 34.5000 34.5000 34.5000 34.5000 34.5000 34.5000 34.5000 34.5000
118.5364 118.5368 118.5371 118.5371 118.5371 118.5368 118.5364 118.5358 118.5352

118.5343

118.5333

118.5322

118.5309

118.5295

118.5278

118.5263

118.5245

118.5228

118.5208 118.5186

118.4773

118.4754

118.4735

118.4718

118.4701

118.4686

118.4672

118.4650

118.4647

118.4637

118.4628

118.4621

118.4614

118.4610

118.4688

118.4684

118.4604

118.4685

118.4688

118.4612

118.4617

118.4624

118.4632

118.4642

118.4653

118.4865

118.4878

118.4694

118.4700

118.4728

118.4744

118.4763

118.5237

118.5255

118.5273

118.5298

118.5308

118.5321
3.67

3.45

3.22

2.78

2.55

.33

2.12

1.90

69

1.49

1.29

1.10

0.82

0.75

0.59

0.44

0.29

0.18

0.05

0.02

0.15

.30

0.45

0.61

.78

0.96

1.15

1.34

1.54

1.75

1.88

3.00

3.28

3.50

3.72

3.83

4.14

4.35

4.56

4.75

4.94

5.13

5.30

5.47

.63

5.78

5.92

.92

5.78

5.63

$5.30 /$

5.13 
ELER771 ANGP-237.7 ANGS-270.0 ATM-270.0 ELEN772 ANG4=241.2 NNG5-270.0 AZMN270.0 FLEN773 ANC4-244.7 ANCS-270.0 AZML270.0 91774 ANC4-248.2 NNCS-270.0 ATML270.0 EM775 ANC4-251.7 ANo5-270.0 AZM-270.0 ELEM776 ANC4-255.1 ANC5-270.0 AZ1ML270.0 ELEN777 ANG4-258.6 ANCS-270.0 ATML270.0 ELEM778 ANG4=262.1 ANC5-270.0 ATML270.0 ELEN79 ANC4-265.6 ANCS-270.0 AZM-270.0 ELEM780 ANC4-269.1 AKCS-270.0 ATML270.8

ELEN81 ANG4-272.B ANOS-270.0 AZM-270.0

ELE782 ANG4-276.1 ANBS-270.0 AZ1M-270.0

ELP783 ANG4-279.6 ANC5-270.0 ATML270.0

ELEN84 ANG4-283.1 NGS-270.0 ATML270.0

ELEN85 ANG4-286.8 AMGS-270.0 AZM-270.0

ELEM786 MNG4-290.1 ANaS-270.0 ATML270.0

ELEM787 ANC4-293.6 ANCS-270.0 NTNm270.0

ELEN788 ANG4-297.1 ANES-270.0 ATMm-270.0

ELEM789 ANG4 300.6 ANeS-270.0 ATM 270.0

ELEN790 ANG4-304.1 NWS-270.0 ATM-270.0

ELEN781 ANG4-307.6 ANG6-270.0 ATML270.0

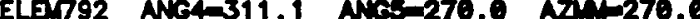

ELET83 ANG4-314.6 ANCS-270.0 ATMm270.0

ELEN794 ANC4-318.1 ANES-270.0 AZML270.0

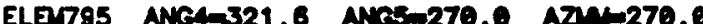

ELEM796 ANG4-325.0 ANES-270.0 AZMM-270.0

RING: 17. 64 ELEVENTS RADIUS- 3.64

ELET97 NGG4 39.6 ANG5= 90.0 ATM 90.0

ELER798 ANC4 42.8 NKES- 90.0 AZMK 90.0

ELDT99 NG4 46.2 NKS 90.0 A7vm 90.0

ELEN800 ANC4 49.5 ANS6- 90.0 AZIM 90.0

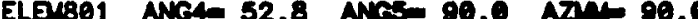

ELEU802 ANG4 56.1 ANCS 00.0 A7ML 90.0

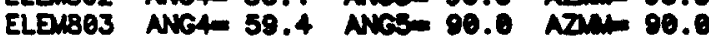

ELEU804 NNC4= 62.8 ANeS= 90.0 ATM 90.0

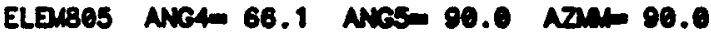

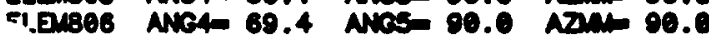

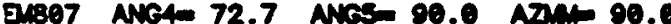

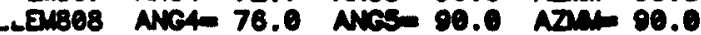

ELEnBeg ANC4 79.3 NAS- 90.0 AZM 00.0

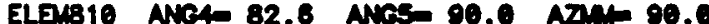

ELEUB11 ANG4 85.9 ANGS 90.0 AZML 90.0

ELENB12 ANG4 89.2 NMES- 90.0 AZM 90.0

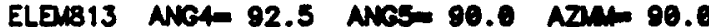

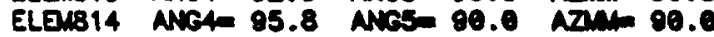

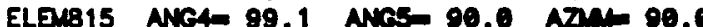

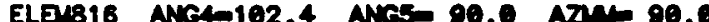

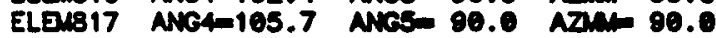

ELEV818 NG4-109.0 NNG5 90.0 ATMW 90.0

ELEN819 ANG4-112.3 NMS 00.0 AZML 90.0

ELEN820 NG4-115.8 NWES 90.8 NZM 90.0

ELEV821 ANC4-118.9 ANo5 00.9 ATM 90.

ELEU822 ANG4-122.2 NNaS- 90.0 AZML 90.0

ELENB23 ANG4-125.5 ANGS 90.0 AZM 90.0

ELEN824 NG4-128.8 NC5 90.0 A7NL 90.0

ELE1825 AKG4-132.1 ANC5 90.0 AZML 90.0

ELEU826 ANG4-135.4 ANC5 90.0 AZM 90.0

ELEV827 ANG4-138.7 NRS $=90.0$ ATML 90.0

ELEN328 ANC4-221.3 ANCS-270.0 AZM-270.0

$\begin{array}{ll}\text { EVENT } & 0 \\ \text { EVENT } & 0 \\ \text { EVENT } & 0 \\ \text { EVENT } & 0 \\ \text { EVENT } & 0 \\ \text { EVENT } & 0 \\ \text { EVENT } & 0 \\ \text { EVENT } & 0 \\ \text { EVENT } & 0 \\ \text { EVENT } & 0 \\ \text { EVENT } & 0 \\ \text { EVENT } & 0 \\ \text { EVENT } & 0 \\ \text { EVENT } & 0 \\ \text { EVENT } & 0 \\ \text { EVENT } & 0 \\ \text { EVENT } & 0 \\ \text { EVENT } & 0 \\ \text { EVENT } & 0 \\ \text { EVENT } & 0 \\ \text { EVENT } & 0 \\ \text { EVENT } & 0 \\ \text { EVENT } & 0 \\ \text { EVENT } & 0 \\ \text { EVENT } & 0 \\ \text { EVENT } & 0\end{array}$

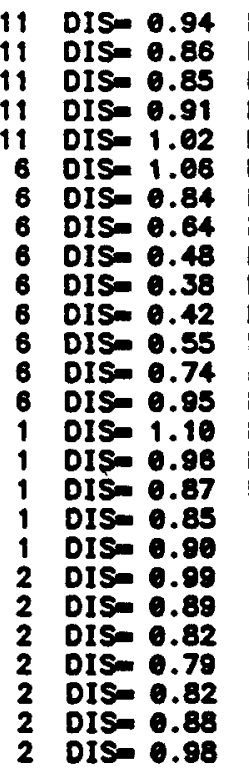

DTIMP-0.11 DTIMP 0.13 OTIMP -0.15 OTIMP-0.17 DTIMPm- -19 OTIMPm 0.05 DTIMP 0.03 DTINP= 0.00 DTIMP -0.02 DTIMP-0.05 DTINo-0.08 DTIMP -0.10 DTIN $=0.13$ DTIMP=0.16 OTINPس 0.03 DTIM DTIMP-0.09 DTIMP-0.12 DTIM-0.15 DTIMP 0.14 OTIMP 0.11 DTINo 0.08 OTIMP 0.05 DTINP= 0.03 DTIMP 0.00 DTIMP -0.02
34.5000

34.5080

34.5600

34.5000

34.5600

34.5000

34.5000

34.5008

34.5000

34.5600

34.5000

34.5000

34.5090

34.5000

34.5000

34.5000

34.5000

34.5000

34.5000

34.5000

34.5000

34.5000

34.5000

34.5000

34.5000

34.5000
198.5334

118.5347

118.5358

118.5367

118.5376

118.5383

118.5388

118.5392

118.5395

118.5398

118.5395

118.5394

118.5390

118.5386

118.5379

118.5372

118.5363

118.5352

118.5341

118.5328

118.5314

118.5298

118.5282

118.5285

118.3246

118.5227
4.94

4.75

4.56

4.35

4.14

3.93

3.72

3.50

3.28

3.06

2.83

2.61

2.39

2.18

1.98

1.75

1.54

1.34

1.15

0.98

0.78

0.61

0.45

0.15

0.02

EVENT 04 DIS- 1.07 DTIMP= $0.03 \quad 34.5000 \quad 118.4732$

EVert 04 DIS- 1.03

EVart 04 DIS- 1.02

EVaNT

EVENT

EVENT

EVENT

EVENT

EVaNT

EVENT

EVENT

EVaNT

EVaT

EVENT

EVENT

EVENT

Evert

EVENT

EVart

EVENT

EVENT

EVENT

EVENT

EVENT

EVENT

EVENT

EVENT

EVENT

EVanT

EVENT

EverT
DIS- 1.02
DISm 1.05

DIS 0.99

DIS 0.82

DIS= 0.69

DIs 0.62

DIS 0.65

DIS 0.75

DIs 0.9

DIS= 0.87

DTIMP 0.05

DTINP 0.08

DTINP 0.11

DTIVP=0.18

DTIMP -0.15

DTIMP $=0.12$

DTIM $=0.09$

DTINP -0.08

DTIMP-0.03

DTIMP=0.00

DTINP -0.11

DTIMP -0.08

DIS- 0.33

DIS- 0.32

DIS 0.18

DTIMP -0.06

DTINP=-0.03

DTINP-0.00

DIS- 0.42

DTINP 0.02

DTIM 0.05

DIS 0.84

DIS= 0.86

DIS= 1.00

DTINP= 0.07

OTINP -0.18

DTINP=0.15

DIS 0.69

DIS 0.62

DTINP $=0.11$

DTIM $=0.09$

DIS 0.75

DIS 0.90

DTIMP -0.08

DTIMP -0.06

DTINP $=0.04$

DIS= 1.03

DIS= 1.02

DTINP= 0.17

DTIMP 0.18

DTIMP 0.20

DTIMP 0.20
DTIMP -0.14

DTILPm-0.13
345000

34.5000

34.5000

34.5000

34.5000

34.5800

34.5000

34.5600

34.5000

34.5060

34.5000

34.5000

34.3000

34.5000

34.5000

34.5000

34.5000

34.5000

34.5000

34.5000

34.5990

34.5000

34.5000

34.5000

34.5000

34.5000

34.5090

34.5000

34.5060

34.5000

34.5000
118.4714

118.4696

118.4688

118.4865 


\begin{tabular}{|c|c|c|c|}
\hline 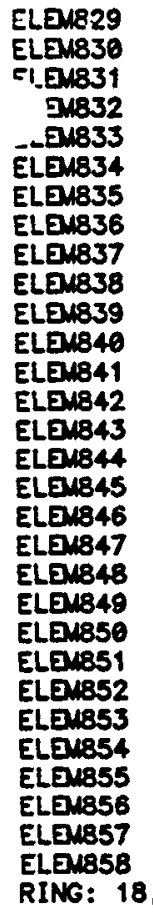 & 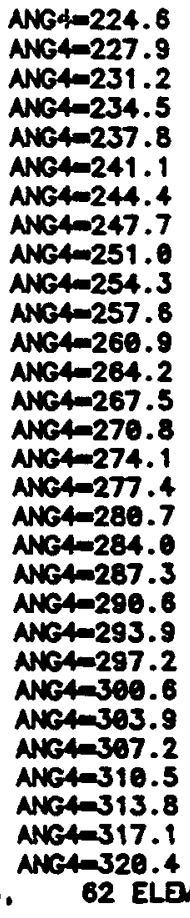 & 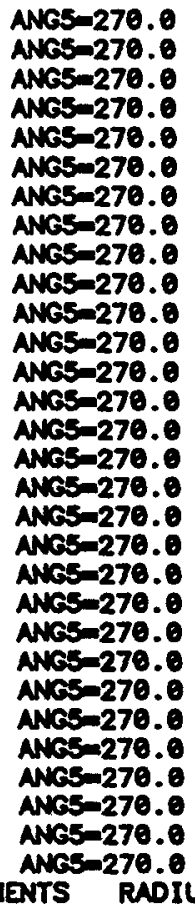 & 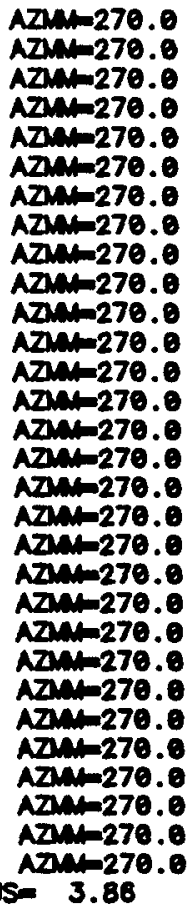 \\
\hline 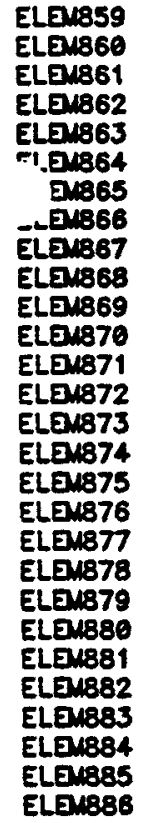 & 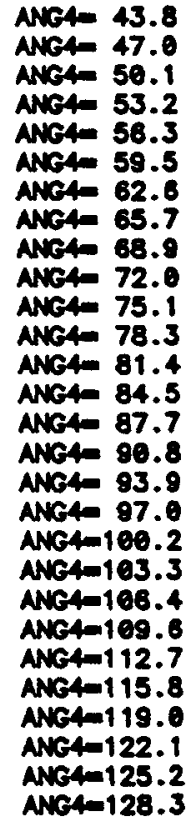 & $\begin{array}{l}90.0 \\
90.0 \\
90.0 \\
90.0 \\
90.0 \\
90.0 \\
90.0 \\
90.0 \\
90.0 \\
90.0 \\
90.0 \\
90.0 \\
90.0 \\
90.0 \\
90.0 \\
90.0 \\
90.0 \\
90.0 \\
90.0 \\
90.0 \\
90.0 \\
90.0\end{array}$ & $\begin{array}{l}90.0 \\
90.0 \\
90.0 \\
90.0 \\
90.0 \\
90.0 \\
90.0 \\
90.0 \\
90.0 \\
90.0 \\
90.0 \\
90.0 \\
90.0 \\
90.0 \\
90.0 \\
90.0 \\
90.0 \\
90.0 \\
90.0 \\
90.0 \\
90.0 \\
90.0 \\
90.0 \\
90.0 \\
90.0 \\
90.0 \\
90\end{array}$ \\
\hline
\end{tabular}

$\begin{array}{lll}\text { EVENT } & 0 & 12 \\ \text { EVENT } & 0 & 12 \\ \text { EVENT } & 0 & 11 \\ \text { EVENT } & 0 & 11 \\ \text { EVENT } & 0 & 11 \\ \text { EVENT } & 0 & 11 \\ \text { EVENT } & 0 & 11 \\ \text { EVENT } & 0 & 11 \\ \text { EVENT } & 0 & 1 \\ \text { EVENT } & 0 & 1 \\ \text { EVENT } & 0 & \\ \text { EVENT } & 0 & 6 \\ \text { EVENT } & 0 & 6 \\ \text { EVENT } & 0 & 6 \\ \text { EVENT } & 0 & \\ \text { EVENT } & 0 & \\ \text { EVENT } & 0 & \\ \text { EVENT } & 0 & \\ \text { EVENT } & 0 & \\ \text { EVENT } & 0 & \\ \text { EVENT } & 0 & \\ \text { EVENT } & 0 & \\ \text { EVENT } & 0 & \\ \text { EVENT } & 0 & \\ \text { EVENT } & 0 & \\ \text { EVENT } & 0 & \\ \text { EVENT } & 0 & \\ \text { EVENT } & 0 \\ \text { EVENT } & 0 \\ \text { EVENT } & 0\end{array}$

12 DIS- 1.02

12 DIS= 1.03

1 DIS= 1.05

11 DIS- 0.90

11 DIS 0.75

11 DIS= 0.65

11 Dis= 0.62

11 DIS= 0.69

11 DIS= 0.83

11 DIS- 1.00

DIS= 0.86

DIS- 0.64

DIS- 0.42

DIS- 0.23

DIS= 0.16

DIS= 0.32

DIS- 0.53

DIS= 0.75

DIS= 0.97

DIS= 0.81

DIS= 0.75

DIS- 0.65

DIS= 0.62

DIS- 0.69

DIS 0.82

DIS- 0.99

DIS- 1.05

DIsw 1.02

DIS- 1.03

DIS- 1.07
DTIMP= 0.18 DTIMP= 0.17 DTIMP -0.04 DTINP -0.08 DTIMP-0.88 DTINP=0.09 DTIMP $=0.11$ DTIMP-6.13 DTIN $=0.15$ DTINP 0.18 OTIND= 0.07 DTIMP 0.05 DTIMP 0.02 DTIMP 0.00 DTIMP- 0.03 DTIMP -0.06 DTINP- -0.68 DTIMP-0.11 DTIMP -0.14 DTIMP -0.00 DTIMP-0.03 DTIMP -0.08 DTIN= $=6.89$ OTIMP $=0.12$ OTIMP -0.15 OTINP-0.18 OTINP= 0.11 OTIMP $=0.08$ OTIMP 0.05 DTIMP 0.03
34.5000 34.5000 34.5000 34.5000 34.5000 34.5000 34.5000 34.5000

34.5000

34.5000

34.5000

34.5000

34.5000

34.5000

34.5000

34.5000

34.5000

34.5000

34.5000

34.5000

34.5090

34.5000

34.5000

34.5069

34.5000

34.5800

34.5000

34.5000

34.5008

34.5000
118.5295

118.5312

18.5327

118.5342

118.5356

118.5368

118.5379

118.5389

118.5397

118.5405

18.5411

118.5415

118.5418

118.5420

118.5420

118.5419

148.5417

118.5413

118.5408

118.5401

118.5393

118.5384

118.5374

118.5362

118.5348

118.5335

118.5328

118.5303

118.5286

118.5268
5.75

5.59

5.42

5.24

5.86

4.87

4.67

4.48

4.28

4.04

3.83

3.81

3.39

3.17

2.94

2.72

2.50

2.28

2.08

1.85

1.64

1.43

1.23

1.04

0.85

0.87

0.49

0.33

0.03

0.05

0.21

0.38

0.55

0.74

0.93

1.12

1.32

1.53

1.74

1.95

2.17

2.39

2.61

2.83

3.06

3.28

3.50

3.72

3.94

4.16

4.37

4.58

4.78

4.98

5.17

5.53 
ELEMB87 ANGA-131.5 ANGS-90.0 AZML 90.0 AMros 00.0 Azanh 90.0

FLEMB

LEM889

ENa90

aus91

ELEM892

ELENG93

ELEU894

ELEM895

ELEMB98

ELEM897

ELEN898

ELEM899

ELEM900

ELEM901

ELEK962

ELEM903

ELEM94

ELEM905

ELAM906

ELEM907

ELEN908

ELEท989

ELEN910

ELDM11

ELJM12

ELEN913

ELEM914

ELEM915

ELEN916

ELDM17

ELEN918 AKC4-316.2 ANBS-270.0 AZM 270.0

RING: 19 ,

10019

ELENA20

ELEM21

-1.EN922

J923

כu924

ELDM925

ELEN926

ELEM927

ELDM28

ELP

ELg4930

ELgMS1

ELEM932

ELEN933

ELEN934

ELEM935

ELDMO36

ELD4937

ELEN938

ELDM939

ELEM940

ELEM941

ELDN942

ELBN943

ELEMS4

ANG5 270.0 AZMM 270.0 ANG5-270.0 ADM 270.0 ANGS 270.0 ATML270.0 NGe-231.7 NGG4-234.8 NGG 237.9 ANG4-241.8 ANG4-244.2 NG4 247.3 ANG4-250.4 ANG4-253.6 NG4 256.7 ANG4-259.8 ANG4-263.0 ANG4-268. NG4-269.2 NNG4-272.3 NG4-275.5 NG4-278.6 ANG4-281.7 ANG5-270.0 ADNW=270.0 ANCS $270.0 \quad$ AZMM 270.0 ANC5 270.0 ATM 270.0 ANGS-270.0 AZINL270.0 ANCS-270.0 AZMM-270.0 ANas -270.0 ATML270.0 ANCS-270.0 AZN 270.0 ANeS-270.0 AZMK270.0 MGS 270.0 AZML270.9 NMGS-270.0 AZNM-270.0 NMCS 270.0 AZM 270.0 ANCS-270.0 AZMW270.0 ANGS-270.0 AZM 270.0 ANGS 270.0 ANML270.0 NMC5-270.0 ATM-270.0 ANGS-270.0 AZMM 270.0 NG4-288.0 ANCS-270.0 ATML270.0 ANG4-291.1 ANGS-270.0 AZMM-270.0 NG4-294.3 ANGS-270.0 AZML270.0 NG4-297.4 ANGS=270.0 ATML270.0 ANG4-300.5 ANGS-270.0 AZML270.0 NM4-303.7 ANGS-270.0 ATM 270.0 NMG4-306.8 ANGS-270.0 AZMM-270.0 NNG4-309.8 ANGS-270.0 AZM 270.0 ANG4-313.0 ANCS-270.0 ATN 270.8 60 ELEMENTS RADIUS- 4.09

NGe4 47.2 AMG5 90.0 AZML 90.0 NMC4 50.2 ANCS- 90.0 ATML 90.0 MNe4- 53.1 ANGS- 90.0 AZML 90.0 ANC4 56.1 ANC5 90.0 AZMW 90.0 ANe4-59.0 ANas- 90.0 ATML 90.0 ANC40 62.0 ANes 90.0 ATML 90.0

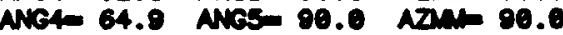
Nuc4 67.9 ANas 90.0 AZMK 90.0 NC4 70.8 ANC5= 90.0 AZM 90.0 ANG4 73.8 ANC5 90.0 AZML 90.0

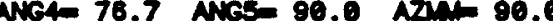
NGS 79.7 ANGS 90.0 AZM 90.0 NGG4 82.8 ANGS 90.0 AZML 80.0 ANG4 85.6 ANBS 90.0 ATM 80.0 ANC40 88.5 ANG5 80.0 AZ1M 90.0 NG4 91.5 NGS 90.0 AZMW 90.0 NC4 94.4 ANG5 90.0 ATM 90.0 ANC4 97.4 ANCS- 90.0 AZML 90.0 AKC4-180.3 AMCS 90.0 ATM 90.0 ANG4-103.3 ANC5- 80.0 ATML 90.0 ANE4-108.2 ANCS 90.0 AZML 90.0 ANG4-109.2 NKS 90.0 AZML 90.0 ANG4l12.1 ANCS 90.0 ATM 90.0 ANG4-115.1 AKC5 90.0 AZML 90.0 ANG4-118.0 ANG5= 90.0 NZML 90.0

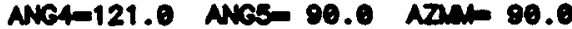

EVENT

EVENT

EVENT

EVENT

EVENT

EVENT

EVENT

EVENT

EVENT

EVENT

EVENT

EVEVT

EVENT

EVENT

EVENT

EVפNT

EVENT

EVENT

EVENT

EVENT

EVENT

EVENT

EVENT

EVENT

EVENT

EVENT

EVENT

EVENT

EVENT

EVIT

EVENT

EVENT

EVaNT

EVENT

EVENT

EVINT

EVENT

EverT

EVENT

EVENT

EVENT

EVENT

EVDNT

EVENT

EVENT

EVeNT

EVENT

EVENT

EVENT

EVENT

EVENT

EVENT

EVart

EVENT

EVENT

EVert

EVENT

15
14
12
11
11
11
11
11
11
11
11
11
6
6
6
6
6
6
6
6
1
1
1
1
1

15
14
12
11
11
11
11
11
11
11
11
11
6
6
6
8
6
6
6
6
6
1
1
1
1
1
1
1
1
1
2
2

DIS= 1.18

DTINP 0.01

34.5800

118.4667

DIS= 1.24 DTIMP $=0.22$

DIS= 1.24

DTIMP $=0.22$

DIS= 1.18

DIS= 0.97

DIS= 0.76

DIS- 0.58

DIS=0.44

DIS= 0.40

DIS= 0.49

DIS- 0.65

DIS- 0.85

DIS= 0.94

DIS- 0.72

DIs- 0.50

DIS- 0.20

DIS= 0.09

DIS $=0.18$

oIS 0.39

DIS- 0.61

DIS 0.83

DIS= 0.95

DIS= 0.75

DIS- 0.56

DIS= 0.43

DIS= 0.40

DIS= 0.50

DIS- 0.67

DIS= 0.86

oIS- 1.08

DIS= 1.25

TINP 0.01

DTINP-0.00

DTIMP- -0.02

DTIMP -0.04

OTIMPm-0.06

DTIMP- 0.07

DTIMP -0.09

DTIMP -0.11

DTIMP -0.13

DTINP 0.12

DTIMP 0.08

DTIMP $=0.07$

DTIMP $=0.04$

DTIMP 0.02

DTINP-0.01

DTIMPm-0.03

DTINP-0.08

DTIMP -0.09

DTIMP 0.05

OTIMP 0.02

DTIMP -0.01

DTIMP=-0.04

DTIMP-0.07

DTIMP -0.09

DTIMP -0.12

DTINP -0.15

DTIMP -0.18

DTINP 0.11

DTINP 0.08

34.5000

34.5000

34.5000

34.5000

34.5000

34.5000

34.5000

34.5000

34.5008

34.5000

34.5000

34.5000

34.5000

34.5000

34.5000

34.5000

34.5000

34.5000

34.5000

34.5000

34.5000

34.5000

34.5000

34.5000

34.5000

34.5000

34.5000

34.5000

34.5000

34.5000

34.5000

118.4683 


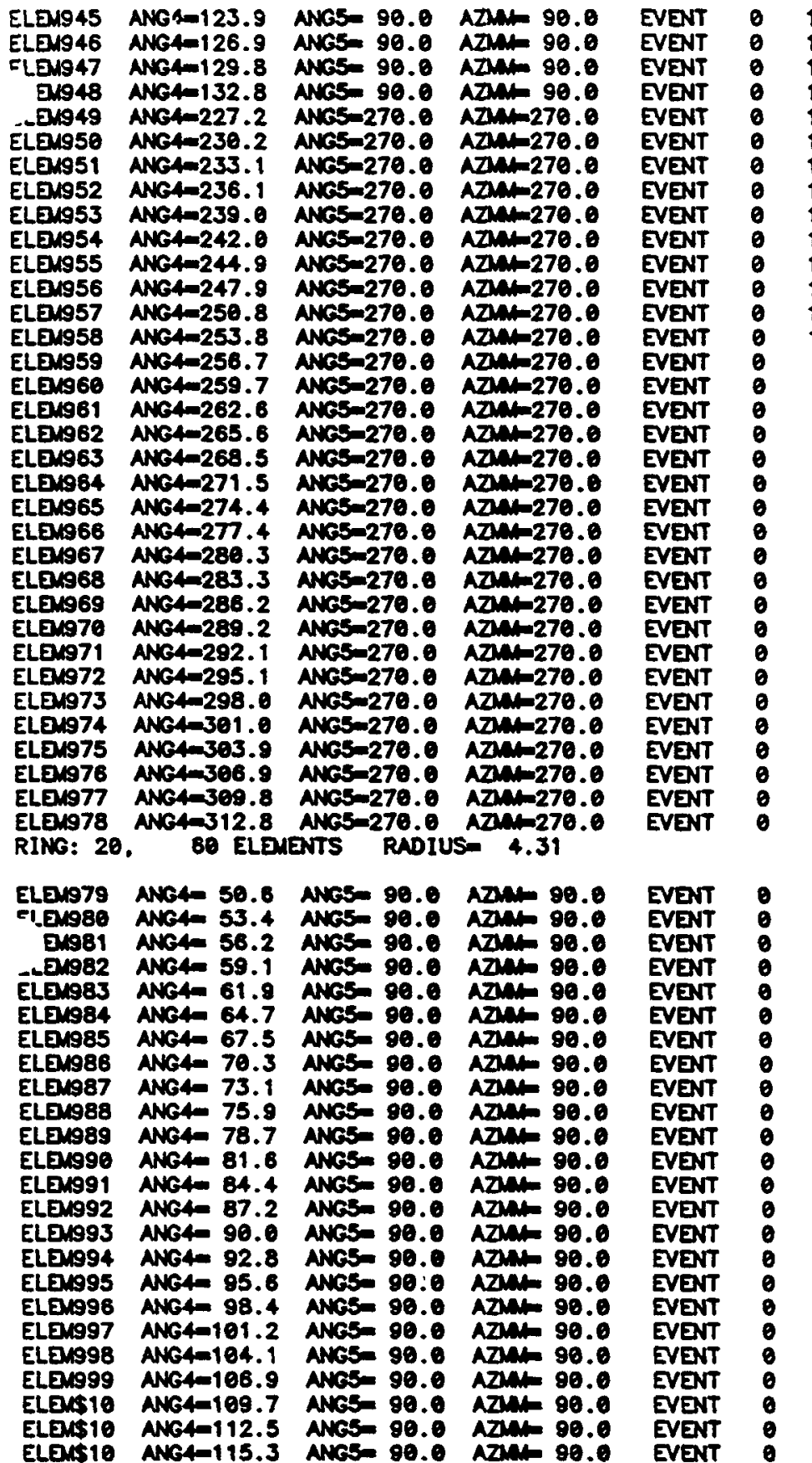

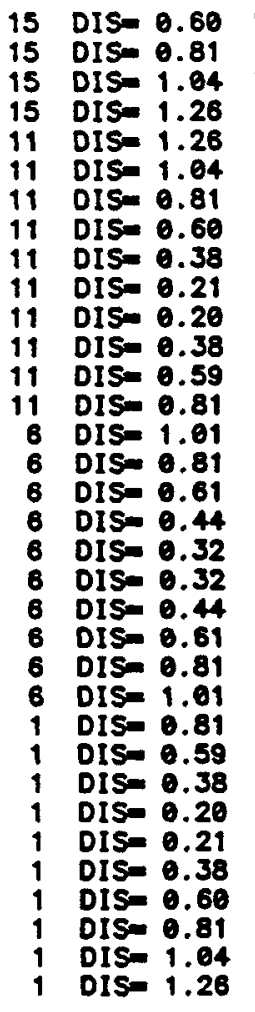

DTIMP= 0.01 DTIMP= 0.03 DTIMP= 0.05 DTIMP= 0.06 DTIMP 0.06 DTIMP $=0.05$ DTIMP $=0.03$ DTIMP 0.01 DTIMP-0.00 DTINP -0.02 DTIMP -0.04 DTIMP-0.08 DTIMP -0.08 DTIMP -0.10 DTIMP 0.16 DTIMP 0.14 DTIMP 0.11 DTIMP 0.09 DTIMP= 0.06 DTINP= 0.04 OTIMP 0.01 DTINP= -0.02 DTIMP-0.04 DTIMP- -0.07 DTIMP= 0.08 DTIM 0.05 DTIMP 0.02 DTIMP-0.01 DTIMP -0.04 DTIMP=-0.07 DTIMP -0.10 DTIMP -0.13 DTINP=-0.15 DTIMP -0.18
34.5000 34.5000 34.5080 34.5000 34.5000 34.5060 34.5000 34.5000 34.5000 34.5000 34.5000 34.5000 34.5000 34.5000 34.5000 34.5000 34.5600 34.5000 34.5000 34.5080 34.5000 34.5000 34.5600 34.5008 34.5060 34.5000 34.5000 34.5000 34.5600 34.5000 34.5000 34.5600 34.5000 34.5000

118.4611 118.4625 118.4640 118.4655 118.5344 118.5360 118.5375 118.5389 118.5402 118.5414 118.5425

118.5435

118.5443

118.5450

118.5457

118.5462

118.5465

118.5468

118.5468

118.3468

118.5468

118.5485

118.5462

118.5457

118.5450

118.5443

118.5435

118.5425

118.5414

118.5462

118.5389

118.5375

118.5380

118.5344

DTIMP-0.12 DTIMP- 0.09 TTIM $=0.86$ DTIMP-0.04 OTIMPm-0.01 DTIMP= 0.02 DTIMP 0.05 DTIMP 0.08 DTIMP 0.18 DTIMP 0.14 DTIMP -0.02 DTIMP 0.0 DTIMP 0.04 DTIMPm 0.06 DTINP 0.09 DTIMP= 0.11 DTINP= 0.14 DTINP 0.16 DTIMP= 0.18 DTIMP -0.08 OTIMP -0.06 OTIMP-0.04 DTIMPD-0.02 DTIMPn 0.00
34.5060 118.4603 $34.5000 \quad 118.4578$ $34.5000 \quad 118.4565$ $34.5009 \quad 118.4554$ $34.5000 \quad 118.4544$ $34.5000 \quad 118.4535$ $34.5000 \quad 118.4527$ $34.5600 \quad 118.4521$ $34.5000 \quad 118.4516$ $34.5090 \quad 118.4511$ $34.5000 \quad 118.4509$ $34.5000 \quad 118.4507$ $34.5900 \quad 118.4508$ $34.5000 \quad 118.4507$ $34.5000 \quad 118.4509$ $34.5000 \quad 118.4511$ $34.5000 \quad 118.4518$ $34.5000 \quad 118.4521$ $34.5000 \quad 118.4527$ $34.5000 \quad 118.4535$ $34.5000 \quad 118.4544$ $34.5000 \quad 118.4554$
5.41

5.59

5.76

5.93

5.93

5.76

5.59

5.41

5.22

5.03

4.83

4.62

4.42

4.20

3.99

3.77

3.55

3.33

3.11

2.89

2.67

2.45

2.23

2.81

1.80
1.58

1.38

1.17

0.97

0.78

0.59

0.24

0.87

0

0.86

1.08

1.26

1.47

1.68

1.98

2.12

2.33

2.56

2.78

3.00

3.22

3.44

3.67

3.88

4.10

4.32

4.53

4.74 
ELEN\$10 ANG:=118.1 ANG5- 90.0 AZMK 90.0 ELEUS10 ANG4-120.9 ANG5 90.0 AZMM 90.0

EI.ENS10 ANG4-123.7 NNG5= 90.0 AZMM 90.0 JS10 ANG4-126.6 ANC5- 90.0 ATMN 90.0 ENS10 ANG4-129.4 ANC5 90.0 AZML 90.0 ELEUS10 ANG4-230.6 ANG5-270.0 AZML-270.0 ELEKS10 ANG4-233.4 ANC5=270.0 AZML270.0 ELEK\$10 ANG4-236.2 ANCS $=270.0$ AZMN-270.0 ELEMS10 ANG4-239.1 ANCS-270.0 AZML-270.0 ELEUS10 ANG4-241.9 ANC5-270.9 ATMK270.0 ELEKS10 ANG4-244.7 ANGS-270.0 AZML270.0 ELONS10 ANG4-247.5 ANG5-270.0 AZMN-270.0 ELELS10 ANC4-250.3 ANCS-270.0 AZMM-270.0 ELDS10 ANC4-253.1 ANCS-270.0 AZML270.0 ELEAS10 ANG4-255.8 ANCS-270.0 AZMM-270.0 ELES10 ANC4m258.7 ANC5 270.8 ATMm270.9 ELENS10 ANG4-261.6 AKOS-270.0 NMML270.0 ELEN\$10 ANG4-264.4 ANGS-270.0 AZMM-270.0 ELESS10 ANG4-267.2 AMCS270.0 ATMM-270.9 ELES10 ANC4-270.0 ANCS-270.0 ATML270.0 ELEN\$10 ANG4-272.8 ANGS-270.0 AZMM-270.0 ELES10 ANG4-275.8 ANC5-270.O ATML270.0 ELEMS10 NG4-278.4 NGS-270.0 AZM-270.0 ELESS10 ANG4-281.2 ANCSm270.0 AZM-270.0 ELES10 MG4m284.1 MNC5=270.0 AZM 270.0 ELEMS10 ANG4-286.8 ANGS-270.0 AZML270.0 ELEN10 ANG4-289.7 ANCS-270.0 AZMm-270.0 ELEMS10 ANG4-292.5 ANC6-270.0 ATMM270.0 ELEUS10 ANG4-295.3 ANG5-270.0 AZML270.0 ELEKS10 ANC4-298.1 ANCS-270.0 AZMm-270.0 ELES10 ANC4-360.9 ANCS 270.0 ATML270.0 ELEN10 ANG4-303.7 ANES-270.0 AZM-270.0 ELEN\$10 ANG4-306.6 ANCS-270.0 ATM-270.0 ELEAS10 ANC4-309.4 ANGS=270.0 AZMM-270.0 RING: 21, 58 ELIMENTS RADIUS- 4.53

ELDS10 ANG4 51.0 ANGS= 80.0 ATM 90.0

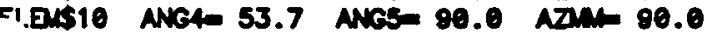
DS10 ANC4 56.4 ANBS- 90.0 AZM 90.0 ANG5 99.0 ATMm 90.0 ELEN10 ANG4- 61.8 ANGS 90.0 AZM 80.0 ELDN10 ANG4 64.5 ANGS 90.0 NZML 90.0 ELENS10 NG4 67.2 NGS- 90.0 AZML 90.0 ELENS10 ANG4 69.9 ANGS-90.0 AZM 90.0 ELENS10 ANG4 72.5 ANGS- 90.0 AZMM 90.0 ELENS10 ANG4- 75.2 ANGS- 90.0 AZML 90.0 ELENS10 ANG4 77.9 ANGS- 90.0 AZMN 90.0 ELENS10 ANG4 80.6 ANG5- 90.0 AZMW 90.0 ELENS10 ANG4 83.3 ANGS- 90.0 AZML 90.0 ELENS10 ANG4 86.0 ANGS- 90.0 AZN 90.0 ELENS10 ANG4 88.7 NGS- 80.0 AZMMm 90.0 ELENS10 ANG4 91.3 ANGS- 90.0 AZMM 90.0 ELELS10 ANG4 84.0 ANG5 90.0 AZML 90.0 ELENS10 ANC4 98.7 NCS5 90.0 AZML 90.0 ELEN\$10 NGG 99.4 NGS= 90.0 AZMM 80.0 ELEN10 NGA-102.1 NGES 80.0 AZML 90.0 ELBS10 NG4-104.8 NGE= 90.0 AZML 89.0 ELEUS10 NGG4107.5 MKC5= 98.0 AZMM 90.0 ELES10 ANG4-110.1 NGS 90.0 AZM 90.0 ELES10 ANG4-112.8 ANCS- 90.0 AVMK 80.0

$\begin{array}{lll}\text { EVENT } & 0 & 15 \\ \text { EVENT } & 0 & 15 \\ \text { EVENT } & 0 & 15 \\ \text { EVENT } & 0 & 15 \\ \text { EVENT } & 0 & 15 \\ \text { EVENT } & 0 & 11 \\ \text { EVENT } & 0 & 11 \\ \text { EVENT } & 0 & 11 \\ \text { EVENT } & 0 & 11 \\ \text { EVENT } & 0 & 11 \\ \text { EVENT } & 0 & 11 \\ \text { EVENT } & 0 & 11 \\ \text { EVENT } & 0 & 11 \\ \text { EVENT } & 6 & 11 \\ \text { EVENT } & 0 & 11 \\ \text { EVENT } & 0 & 6 \\ \text { EVENT } & 0 & 6 \\ \text { EVENT } & 0 & 6 \\ \text { EVENT } & 0 & 6 \\ \text { EVENT } & 0 & 6 \\ \text { EVENT } & 0 & 6 \\ \text { EVENT } & 0 & 6 \\ \text { EVENT } & 0 & 6 \\ \text { EVENT } & 0 & 6 \\ \text { EVENT } & 0 & 1 \\ \text { EVENT } & 0 & 1 \\ \text { EVENT } & 0 & 1 \\ \text { EVENT } & 0 & 1 \\ \text { EVENT } & 0 & 1 \\ \text { EVENT } & 0 & 1 \\ \text { EVENT } & 0 & 1 \\ \text { EVENT } & 0 & 1 \\ \text { EVENT } & 0 & 1 \\ \text { EVENT } & 0 & 1 \\ \text { EV } & & \end{array}$

EVENT EVפT

EVENT

EVENT

EVENT

EVENT

EVENT

EVENT

EVENT

EVENT

EVENT

EVENT

EVETT

EVart

EVENT

EVENT

EVENT

EVENT

EVENT

EVENT

EVIT

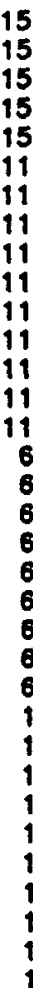

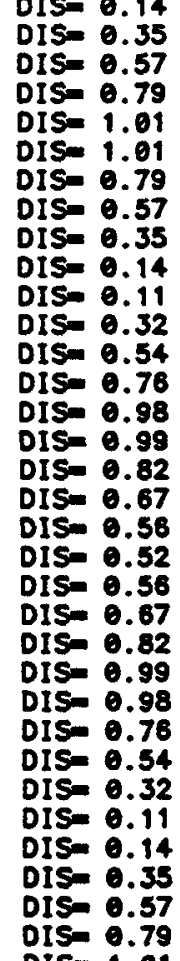

DIS= 1.01
DTIMP 0.02 DTINP= 0.04
DTINP= 0.05 DTINa 0.07 DTIMP 0.09 DTIMP= 0.09 DTIMP 0.07 DTIMP 0.05 DTIMP 0.04 DTIMP $=0.02$ DTIMP 0.00 DTINP -0.02 DTIMP -0.04 OTIMP -0.08 DTIMP -0.08 OTIMP 0.18 DTINP= 0.18 DTIMP= 0.14 DTIMP 0.11 DTIMP= 0.09 DTIMP 0.06 DTINP 0.04 DTIMP= 0.01 DTIMP -0.02 DTIMP 0.14 DTIMP 0.11 DTIMP= 0.08 DTIMP 0.05 DTIMP= 0.02 DTIMP -0.01 DTIM $=0.04$ DTIMP $=0.08$ DTIMP-0.09 DTIMP=0.12
34.5000 34.5000 34.5000 34.5000 34.5000 34.5000 34.5000 34.5600 34.5600 34.5000 34.5000 34.5000 34.5000 34.5000 34.5000 34.5008 34.5000 34.5000 34.5000

34.5000

34.5000

34.5000

34.5000

34.5000

34.5000

34.5000

34.5000

34.5000

34.5000

34.5000

34.5000

34.5000

34.5000

34.5000
118.4565 118.4578 118.4589

118.4603

118.4618

118.5382

118.5397

118.5416

118.5423

118.5435

118.5446

118.5458

118.5465

118.5472

118.5479

118.5484

118.5488

118.5491

118.5493

118.5484

118.5493

118.5491

118.5488

118.5484

118.5470

118.5472

118.5465

118.5456

118.5446

118.5435

118.5423

118.5410

118.5397

118.5382
5.14

5.33

5.52

5.70

5.88
5.88

5.70

5.52

5.33

5.14

4.94

4.74

4.53

4.32

4.10

3.86

3.67

3.22

3.00

2.78

8.58

2.33

2.12

8.88

1.68
1.47

1.26

1.06

0.86

0.48

0.30

0.12

0.01

0.18

0.37

0.58

0.75

0.85

1.15

1.36

1.57

1.79

2.00

2.22

2.44

2.67

2.89

3.11

3.33

3.58

3.78

4.00

4.21

4.43

4.84 
ELEN10 ANG:=115.5 ANG5 90.0 AZMN 90.0 ELEN\$10 ANG4-118.2 ANG5- 90.0 AZML 90.0 FLEMS10 ANG4-120.9 ANG5 90.0 AZML 90.0

BNS10 ANG4-123.6 ANG5 90.0 ATML 90.0

-EM\$10 ANG4-126.3 ANGS 90.0 AZM 90.0

ELENS10 ANG4-129.6 ANGS- 90.0 AZNL 90.0

ELEMS10 ANGA-231.0 ANGS-270.0 AZML270.0

ELDKS10 ANG4-233.7 ANGS-270.0 AZMM-270.0

ELEK\$10 ANG4-236.4 MNGS-270.8 AZM-270.0

ELENS10 ANG4-239.1 ANG5-270.0 AZNM-270.0

ELEMS10 ANG4-241.8 ANGS-270.0 NZNW-270.0

ELEMS10 ANG4-244.5 ANGS-270.0 AZNK270.0

ELEN\$10 ANG4-247.2 ANGS-270.0 AZMW270.0

ELEM\$10 ANG4-249.9 ANG5-270.0 AZNM-270.0

ELEU\$10 ANG4-252.5 ANGS-270.0 AZML270.0

ELENS10 ANG4-255.2 ANG5=270.0 AZMM-270.0

ELEN\$10 ANG4-257.9 ANGS-270.6 AZM-270.6

ELENS10 ANG4-260.6 ANGS-270.0 AZMU270.0

ELEUS10 ANG4-263.3 ANGS-270.0 AZMW-270.0

ELBN\$10 ANG4-266.0 ANGS=270.0 AZNW-270.0

ELES10 ANG4-268.7 ANG5-270.0 AZML270.0

ELENS10 ANG4-271.3 ANGS-270.0 AZNM-270.0

ELENS10 ANG4-274.0 ANGS-270.0 AZNK270.0

ELEST10 ANG4-276.7 ANG6-270.0 ATML270.0

ELEN\$10 ANG4-279.4 ANGS-270.0 AZNW-270.0

ELENS10 ANG4-282.1 ANCS-270.0 AZNM-270.0

ELENS10 ANG4-284.8 ANGS-270.0 ATML270.0

ELENS10 ANG4-287.5 ANG5-270.0 AZML270.0

ELEN\$10 ANG4-290.1 ANGS=270.0 AZMW270.0

ELENS10 ANG4=292.8 ANG5=270.0 AZML270.0

ELENS10 ANG4-295.5 ANGS-270.0 AZMN-270.0

ELENS10 ANG4-298.2 ANGS-270.0 AZML270.0

ELENS10 ANG4-300.9 ANG5-270.0 ATML270.0

ELENS10 ANG4-303.6 ANGS-270.0 AZMN270.0

ELEN\$10 ANG4-306.3 ANGS 270.0 AZMM-270.0

ELEUS10 ANG4m309.0 ANG5-270.0 ATML270.0

RING: 22. 60 ELEMaNTS RADIUS- 4.76

IMS10 ANG4 53.6 ANGS- 90.0 AZML 90.0 CDNS10 ANG4 56.2 ANGS- 90.0 AZIML 80.0 ELDN10 ANG4 58.7 ANGS 80.0 AZNM 90.0 ELDS11 ANG4- 61.3 NGS- 90.0 ATN 90.0 ELENS11 ANG4 63.8 ANGS= 90.0 AZML 80.0 ELEU\$11 ANG4= 66.4 ANGS- 90.0 AZNm 90.0 ELENS11 ANG46 68.9 NGS= 90.0 AZMm 90.0 ELEUS11 ANG4 71.5 ANGS 90.0 AZMK 90.0 ELENSII ANG4 74.0 ANGS 90.0 AZMK 90.0 ELEST11 ANG4 78.6 ANCS= 90.0 ATM 90.0 ELENS11 ANG4 79.1 ANGS- 90.0 AZML 90.0 ELENS11 ANG4- 81.7 ANG5 90.0 AZML 90.0 ELENS11 ANG4 84.3 ANG5- 90.0 ATM 90.0 ELEN\$11 ANG4- 86.8 ANGS- 90.0 AZML 90.0 ELENS11 ANG4- 89.4 NNC5- 90:0 AZML 90.0

ELES11 ANG4 91.9 ANGS 90.0 ATMm 90.0

ELEN511 ANG4- 94.5 NNG5-90.0 AZML 90.0

ELEK\$11 ANG4- 97.0 ANGS 90.0 AZML 90.0

ELES19 ANG4- 99.6 ANCS- 80.0 AZN 90.0

ELENS11 ANG4-102.1 ANG5- 90.0 AZM 90.0

ELES11 ANG4-104.7 ANGS= 90.0 AZM 90.0

ELES11 NG4-107.2 MNGS- 80.0 ADMh 90.0

$\begin{array}{ll}\text { EVENT } & 0 \\ \text { EVENT } & 0 \\ \text { EVENT } & 0 \\ \text { EVENT } & 0 \\ \text { EVENT } & 0 \\ \text { EVENT } & 0 \\ \text { EVENT } & 0 \\ \text { EVENT } & 0 \\ \text { EVENT } & 0 \\ \text { EVENT } & 0 \\ \text { EVENT } & 0 \\ \text { EVENT } & 0 \\ \text { EVENT } & 0 \\ \text { EVENT } & 0 \\ \text { EVENT } & 0 \\ \text { EVENT } & 0 \\ \text { EVENT } & 0 \\ \text { EVENT } & 0 \\ \text { EVENT } & 0 \\ \text { EVENT } & 0 \\ \text { EVENT } & 0 \\ \text { EVENT } & 0 \\ \text { EVENT } & 0 \\ \text { EVENT } & 0 \\ \text { EVENT } & 0 \\ \text { EVENT } & 0 \\ \text { EVENT } & 0 \\ \text { EVENT } & 0 \\ \text { EVENT } & 0 \\ \text { EVENT } & 0 \\ \text { EVENT } & 0 \\ \text { EVENT } & 0 \\ \text { EVENT } & 0 \\ \text { EVENT } & 0 \\ \text { EVENT } & 0 \\ \text { EVENT } & 0 \\ \text { VVIT } & \end{array}$

15

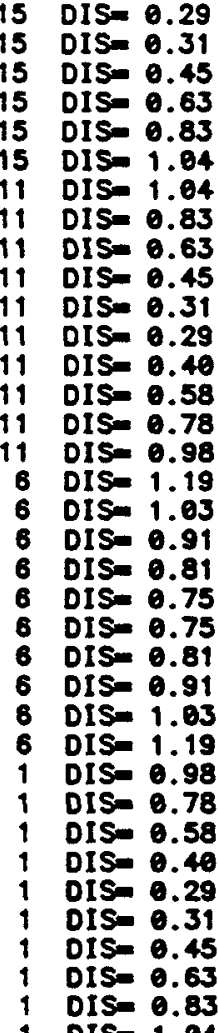

EVENT

EVENT

EVET

EVENT

EVENT

EVENT

EVENT

EVent

EVENT

EVENT

EVENT

EVENT

EVENT

EVENT

EVENT

EVENT

EVENT

EVeNT

EVENT

EVENT

DIS= 0.96 DIS= 0.79 DIS= 0.84

DIS= 0.53

DIS $=0.50$

DIS- 0.55

DIS= 0.67

DIS= 0.83

DIS= 1.00

DIS 1.19

10 DIS- 1.29

10 DIS 1.17

10 DIS= 1.07

10 DISE 1.00

10 DIS 0.97

10 DIS= 0.98

10 DIS= 1.03

10 DIS= 1.11

10 DIS- 1.22

15 DIS- 1.28

15 DISn 1.10
DTINP= 0.04 DTIMP= 0.06 DTIMP $=0.08$ DTIMP= 0.10 DTIMP 0.11 DTINP 0.13 DTIMP= 0.13 DTIMP= 0.11 DTIMP 0.10 DTIMP 0.08 DTINP= 0.03 DTIMP 0.01 DTINP-0.01 DTIMP -0.03 DTIMP $=0.23$ DTINP= 0.21 DTINP= 0.16 DTIMP 0.14 DTINP 0.11 DTIMP 0.09 DTIMP 0.06 DTIMP 0.04 DTIMP 0.01 DTIMP= 0.17 DIINP 0.14 DTIMP 0.11 DTIMP 0.08 DTINP 0.05 DTINP 0.03 DTIMP -0.00 DTIMP-0.03 DTIMP $=0.06$ DTIMP $=0.08$ DTIMP $=0.06$ DTINP= 0.04 DTIMP $=0.19$
34.5000 34.5000 34.5000 34.5000 34.5600 34.5000

34.5000

34.5008

34.5000

34.5000

34.5000

34.5000

34.5000

34.5000

34.5000

34.5000

34.5000

34.5000

34.5000

34.5000

34.5000

34.5600

34.5000

34.5000

34.5000

34.5000

34.5000

34.5000

34.5000

34.5000

34.5000

34.5000

34.5000

34.5600
34.5000

34.5000

118.4532

118.4543

118.4555

118.4568

118.4582

118.4597

118.5403

118.5418

118.5432

118.5445

118.5457

118.5468

118.5477

118.5486

118.5494

118.5501

118.5507

118.5511

118.5515

118.5517

118.5518

118.5518

118.5517

118.5515

118.5511

118.5507

118.5501

118.5494

118.5486

118.5477

118.5468

118.5457

118.5445

118.5432

118.5418

118.5403

TIMP- 0.02

15 DIS 0.81 DTINP= 0.03 DTIM 0.08 DTIMP 0.08 DTIMP 0.11 DTIMP 0.14 DTINP 0.17 DTINP= 0.20 DTIN= 0.22 DTIMP 0.06 DTINP 0.09 DTIMP 0.11 DTIMPa 0.13 DTINo 0.16 DTIMP 0.18 DTINPD 0.21 DTINP= 0.23 DTIMP 0.25 DTINP 0.02

DTIMP= 0.01 DTINP= 0.02
118.4583 $34.3000 \quad 118.4538$ $34.5000 \quad 118.4524$ $34.5000 \quad 118.4513$ $34.5000 \quad 188.4503$ $34.5000 \quad 118.4494$ $34.5000 \quad 118.4485$ $34.5000 \quad 118.4472$ $34.5000 \quad 118.4467$ $34.5000 \quad 118.4463$ $34.5000 \quad 118.4460$ $34.5600 \quad 118.4458$ $34.5600 \quad 118.4457$ $34.5000 \quad 118.4458$ $34.5000 \quad 118.4459$

$34.5000 \quad 118.4465$ $34.5000 \quad 118.4469$ $34.5000 \quad 118.4475$ $34.5000 \quad 118.4482$ $34.5000 \quad 118.4478$ $34.5000 \quad 118.4461$

5.95

5.25

5.44

5.83

5.82

5.99

5.82

5.63

5.44

5.25

4.85

4.64

4.43

4.21

4.00

3.58

3.33

3.11

2.89

2.67

2.44

2.22

2.00

1.79

1.57

1.36 
ELENS11 ANG:=109.8 ANG5 90.0 AZM 90.0 ELEMS11 ANG4-112.3 ANGS= 90.0 AZMM 90.0 ELOMS11 ANG4=114.9 ANGS- 90.0 AZMM 90.0 3K511 ANG4-117.4 ANG5 90.0 AZML 90.0 -EEKS11 ANG4=120.0 ANGS 90.0 AZML 90.0 ELEN11 ANG4-122.8 ANG5- 90.0 AZMM 90.0 ELENS11 ANG4-125.1 MGG5 90.0 AZML 90.0 ELENS11 ANG4-234.9 ANG5-270.0 AZMN270.0 ELDKS11 ANG4-237.4 ANGS-270.0 AZNK-270.0 ELES11 NG4-240.0 NGS 270.0 AZML270.0 ELEMS19 ANG4-242.6 NGS-270.0 AZMM-270.0 ELION11 ANG4-245.1 ANG5-270.0 AZMW-270.0 ELDNS11 ANG4=247.7 ANGS=270.0 AZML270.0 ELDA 11 NGG-250.2 NGS-270.0 AZML270.0 ELENS11 ANG4-252.8 NKS-270.0 AZMW-270.0 ELGK11 ANG4-255.3 NCS-270.0 AZM-270.0 ELEM11 NG4-257.9 NMES-270.0 AZMM-270.0 ELDKS11 ANG4-260.4 ANCS-270.0 AZMM-270.0 ELDS11 NG4-263.0 MC5-270.0 ATML270.0 ELENS11 ANG4-265.5 NKO5-270.0 AZMW-270.0 ELENS11 ANG4-268.1 NGS-270.0 AZML270.0 ELEAS11 NG4-270.6 ANCS-270.0 A7Mm270.0 ELES11 NGG-273.2 NNOS-270.0 AZMm270.0 ELENS11 NMG4-275.7 NNG5=270.0 AZML270.0 ELENS11 NGC4-278.3 ANGS=270.0 AZML270.0

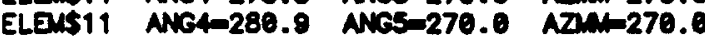
ELENS11 NG4-283.4 MGS-270.0 ATM-270.0 ELENS11 NGG4288.0 ANCS-270.0 AZML270.0 ELEUS11 ANG4-288.5 NKG5=270.0 AZMM-270.0 ELEUS11 ANG4=291.1 MNOS-270.0 AZML270.0 ELELS11 ANG4-293.8 NWGS-270.0 AZMm-270.0 ELEUS11 ANG4=296.2 ANGS-270.0 AZML270.0 ELEN511 ANG4=298.7 ANGS-270.0 AZML270.0 ELOUS11 ANG4-301.3 ANGS-270.0 AZMW-270.0 ELEM11 AKG4-303.8 MGS-270.0 AZMM-270.0 ELDS11 AMG4-306.4 AKC5=270.0 A7MM-270.0 RING: 23, 58 ELEMENTS RADIUSE 4.88

BST11 ANG4- 56.3 NKaS 90.0 AZMW 90.0 -LEX11 NG4 58.8 ANES- 90.0 AZM 00.0 ELES11 ANG4 61.2 ANCS- 90.0 AZML 09. ELEST11 NGS 63.7 ANG5 90.0 AZML 80.0

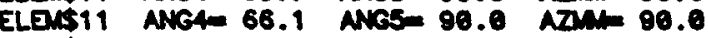
ELDS11 NG4 68.6 ANC5 90.0 AZM 90.0 ELES11 ANC4 71.0 ANCS- 90.0 ATM 90.0 ELENS11 ANG4-107.8 ANG5- 90.0 AZN 90.0 ELDKS11 ANG4=110.2 ANGS- 80.0 AZML 90.0 ELENS11 ANG4-112.7 ANGS- 90.0 AZML 90.0 ELEN11 ANG4-115.1 ANGS 90.0 AZML 90.0 ELEKS11 ANG4=117.6 ANG5= 90.0 AZML 90.0 ELDSS11 ANG4=120.0 ANG5= 90.0 AZMm 80.0 ELEN511 ANG4-122.4 ANGS- 90.0 AZML 80.0 ELENS11 ANG4-124.9 ANGS- 90.0 AZM 80.0 ELEN\$11 ANG4-235.1 ANC5m270.0 AZMM-270.0 ELENS11 ANG4-237.6 ANG5=270.0 AZMM-270.0 ELEUS11 ANG4-240.0 ANG5-270.0 AZN-270.0 ELEN\$11 ANG4-242.4 ANCS=270.0 NZML270.0 ELENS11 ANG4-244.9 ANCS=270.0 AZNK=270.0 ELEXS11 ANG4=247.3 ANCS=270.0 ATML270.0 ELENS11 ANG4-248.8 NKSS-270.0 AZMN-270.0

EVENT
EVENT
EVENT
EVENT
EVENT
EVENT
EVENT
EVENT
EVENT
EVENT
EVENT
EVENT
EVENT
EVENT
EVENT
EVENT
EVENT
EVENT
EVENT
EVENT
EVENT
EVENT
EVENT
EVENT
EVENT
EVENT
EVENT
EVENT
EVENT
EVENT
EVENT
EVENT
EVENT
EVENT
EVENT
EVENT

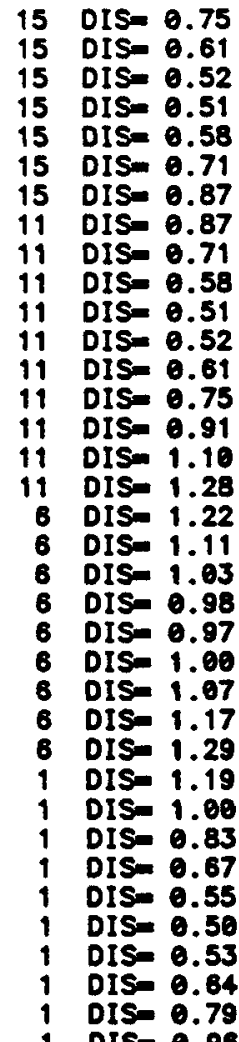

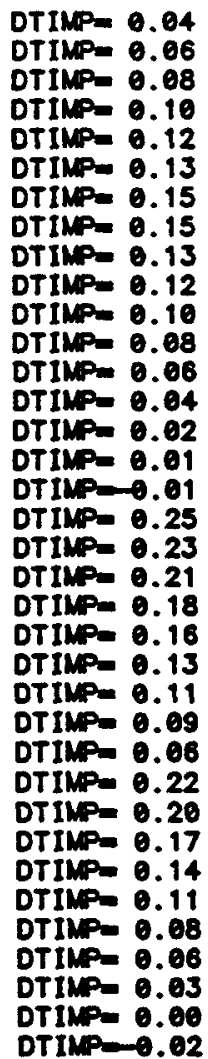

34.5000

34.5000

34.5000

34.5000

34.5000

34.5000

34.5000

34.5000

34.5000

34.5000

34.5000

34.5000

34.5000

34.5000

34.5000

34.5000

34.5000

34.5000

34.5000

34.5000

34.5000

34.5000

34.5000

34.5000

34.5000

34.5000

34.5000

34.5000

34.5600

34.5000

34.5000

34.5000

34.5000

34.5000

34.5000

34.5000

118.4498

118.4508

118.4518

118.4530

118.4543

118.4556

118.5444

118.5457

118.5470

118.5482

118.5492

118.5502

118.5511

118.5518

118.5525

118.5531

118.5535

118.5538

118.5541

118.5542

118.5543

118.5542

118.5540

118.5537

118.5533

118.5528

118.5522

118.5515

118.5566

118.5497

118.5487

118.5476

118.5464

118.5451

118.5437

4.69

.89

5.10

5.30

5.49

5.68

8.87

5.87

5.68

5.49

5.30

5.10

4.89

4.69

4.48

4.28

4.05

3.83

3.61

3.39

3.17

2.94

2.72

2.50

2.28

2.06

1.84

1.63

1.42

1.21

1.00

0.80

.60

0.41

0.23

0.04

EVENT

EVENT

EVENT

EVENT

EVant

EVENT

EVENT

EVENT

EVENT

EVENT

EVENT

EVENT

EVENT

EVENT

EVENT

EVENT

EVENT

EVENT

EVENT

EVENT

EVENT
5 DISn 0.95

5 DIS- 0.83

5 DIS= 0.75

5 DIS 0.73

5 DIS- 0.76

5 DIS- 0.84

5 DIS 0.98

15 DIS= 1.03

15 DIS= 0.90

15 DIS= 0.80

15 DIS- 0.74

15 OIS= 0.73

15 DIS- 0.78

15 DIS- 0.88

15 DIS- 1.01

11 DIS 1.01

11 DIS- 0.88

11 DISm 0.78

11 DIS 0.73

11 DIS- 0.74

11 DIS= 0.80

11 DIS- 0.90
DTINP= 0.04 DTINP= 0.07 DTINP= 0.09 DTIMP $=0.12$ DTIMP 0.15 DTIMP 0.18 DTINP= 0.28 DTIMP 0.07 DTINP 0.09 DTINP= 0.11 DTIMP $=0.12$ DTIMP 0.14 DTINP 0.18 DTIMP $=0.17$ DTIMP 0.18 DTIM $=0.19$ DTINP 0.17 DTINP 0.16 DTIMP 0.14 DTIMP 0.12 DTIMP 0.11 DTIMP 0.09
34.5000 34.5600 34.5600

34.5000

34.5060

34.5000

34.5000

34.5000

34.5000

34.5000

34.5000

34.5000

34.5000

34.5008

34.5000

34.5000

34.5000

34.5000

34.5000

34.5000

34.5000

34.5000
118.4528

118.4515

118.4503

118.4492

118.4481

118.4472

118.4464

118.4460

118.4468

118.4476

118.4486

118.4497

118.4509

118.4521

118.4535

118.5465

118.5479

118.5491

118.5503

118.5523

118.5532
118.5513

0.11

0.38

0.49

0.69 
ELEN\$1 ANGA $=252.2$ ANG5-270.0 AZML270.0 ELENS11 ANG4-286.5 ANG5 270.0 AZMK 270.0 5. DUS11 ANG4-289.0 ANG5 270.0 AZML270.0 GUS11 ANG4-291.4 ANGS-270.0 AZML270.0 -DNS11 ANG4-293.9 ANC5-270.0 AZNL=270.0 ELENS11 ANG4-296.3 ANC5-270.0 ATML270.0 ELEN\$11 ANG4-298.8 ANGS-270.0 AZML-270.0 ELEN11 ANG4-301.2 ANG5-270.0 AZMK=270.0 ELEUS11 ANG4-303.7 ANGS-270.0 AZMK270.0 RING: 24, 31 ELEMENTS RADIUSm 5.21

ELEN11 ANG4- 58.8 ANG5- 90.0 AZMN 90.0 ELEN11 ANG4 61.2 ANGS 90.0 AZMM 90.0 ELENS11 ANG4 63.5 ANCS= 90.0 AZML 90.0

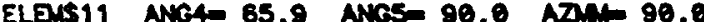

ELENS11 ANG4-115.3 NNGS 90.0 AZMm 90.0

ELEK\$1 ANG4-117.6 ANGS= 90.0 AZMK 90.0

ELEAS11 ANG4-120.0 ANG5- 80.0 ATMK 90.0

ELENS11 NCAm122.4 NNGS 80.0 AZML 90.0

ELEAII ANG4-237.6 ANGS-270.0 AZMML270.0

ELEN11 ANC4-240.0 NNG5-270.0 AZM-270.0

ELEN\$11 ANG4-242.4 ANG5=270.0 AZMM-270.0

ELEN11 ANC4-244.7 NWOS=270.0 AZML270.0

ELEK11 NMC4-294.1 NNC5-270.0 AZM-270.0

ELENS11 ANC4-296.5 NMS5-270.0 AZM-270.6

ELENS12 ANG4-298.8 ANCS-270.0 ATM 270.0

ELENS12 ANG4-301.2 ANCS-270.0 AZML270.0 RING: 25. 16 ELEMENTS RADIUS 5.43

ELEN12 ANG4 58.9 ANG5 90.0 AZM 90.8 ELENS12 ANG4- 61.1 NNG5 90.0 AZML 90.0 ELDS12 ANG4=120.0 ANG5= 90.0 AZMK 90.0 ELES12 NG4-240.0 NGS-270.0 AZMM270.0 ELELS12 ANG4-298.9 ANGS-270.0 AZNM270.0 ELDS12 ANG4-301.1 ANGS-270.0 AZM-270.0 RING: 26, 6 ELEIENTS RADIUS- 5.68

\begin{tabular}{|c|c|c|c|c|c|c|c|c|}
\hline $\begin{array}{l}\text { EVENT } \\
\text { EVENT } \\
\text { EVENT } \\
\text { EVENT } \\
\text { EVENT } \\
\text { EVENT } \\
\text { EVENT } \\
\text { EVENT } \\
\text { EVENT }\end{array}$ & $\begin{array}{l}0 \\
0 \\
0 \\
0 \\
0 \\
0 \\
0 \\
0 \\
0\end{array}$ & $\begin{array}{l}11 \\
1 \\
1 \\
1 \\
1 \\
1 \\
1 \\
1 \\
1\end{array}$ & 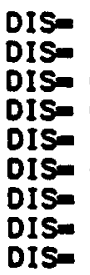 & $\begin{array}{l}1.03 \\
1.11 \\
0.96 \\
0.84 \\
0.76 \\
0.73 \\
0.75 \\
0.83 \\
0.95\end{array}$ & $\begin{array}{l}\text { DTIMP } \\
\text { DTIMP= } \\
\text { DTIMP } \\
\text { DTIMP } \\
\text { DTINP= } \\
\text { DTINP= } \\
\text { DTINP= } \\
\text { DTIMP= } \\
\text { DTIMP= }\end{array}$ & $\begin{array}{l}0.07 \\
0.23 \\
0.20 \\
0.18 \\
0.15 \\
0.12 \\
0.09 \\
0.07 \\
0.04\end{array}$ & $\begin{array}{l}34.5000 \\
34.5000 \\
34.5000 \\
34.5000 \\
34.5000 \\
34.5000 \\
34.5000 \\
34.5000 \\
34.5000\end{array}$ & $\begin{array}{l}118.5540 \\
118.5544 \\
118.5536 \\
118.5528 \\
118.5518 \\
118.5568 \\
118.5497 \\
118.5485 \\
118.5472\end{array}$ \\
\hline $\begin{array}{l}\text { EVENT } \\
\text { EVENT } \\
\text { EVENT } \\
\text { EVENT } \\
\text { EVENT } \\
\text { EVENT } \\
\text { EVENT } \\
\text { EVENT } \\
\text { EVENT } \\
\text { EVENT } \\
\text { EVENT } \\
\text { EVENT } \\
\text { EVENT } \\
\text { EVENT } \\
\text { EVENT } \\
\text { EVENT }\end{array}$ & $\begin{array}{l}0 \\
0 \\
0 \\
0 \\
0 \\
0 \\
0 \\
0 \\
0 \\
0 \\
0 \\
0 \\
0 \\
0 \\
0 \\
0\end{array}$ & $\begin{array}{r}5 \\
5 \\
5 \\
5 \\
15 \\
15 \\
15 \\
15 \\
11 \\
11 \\
11 \\
11 \\
1 \\
1 \\
1 \\
1\end{array}$ & $\begin{array}{l}\text { DIS- } \\
\text { DIS= } \\
\text { DIS= } \\
\text { DIS= } \\
\text { DIS= } \\
\text { DIS= } \\
\text { DIS= } \\
\text { DIS= } \\
\text { DIS= } \\
\text { DIS= } \\
\text { DIS= } \\
\text { DIS= } \\
\text { DIS= } \\
\text { DIS- } \\
\text { DIS- } \\
\text { DIS= }\end{array}$ & $\begin{array}{l}1.03 \\
0.97 \\
0.95 \\
0.97 \\
0.96 \\
0.96 \\
1.00 \\
1.08 \\
1.08 \\
1.00 \\
0.96 \\
0.98 \\
0.97 \\
0.95 \\
0.97 \\
1.03\end{array}$ & 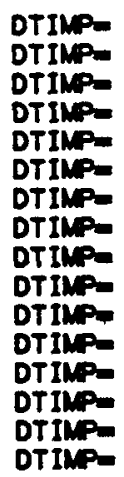 & $\begin{array}{l}0.11 \\
0.13 \\
0.18 \\
0.19 \\
0.17 \\
0.18 \\
0.20 \\
0.22 \\
0.22 \\
0.20 \\
0.18 \\
0.17 \\
0.19 \\
0.16 \\
0.13 \\
0.11\end{array}$ & $\begin{array}{l}34.5000 \\
34.5000 \\
34.5000 \\
34.5000 \\
34.5000 \\
34.5000 \\
34.5000 \\
34.5000 \\
34.5000 \\
34.5000 \\
34.5000 \\
34.5000 \\
34.5000 \\
34.5000 \\
34.5000 \\
34.5000\end{array}$ & $\begin{array}{l}118.4494 \\
118.4482 \\
118.4470 \\
118.4480 \\
118.4465 \\
118.4476 \\
118.4488 \\
118.4500 \\
118.5580 \\
118.3512 \\
118.5524 \\
118.5535 \\
118.5540 \\
118.5529 \\
118.5518 \\
118.5508\end{array}$ \\
\hline $\begin{array}{l}\text { EVENT } \\
\text { EVENT } \\
\text { EVENT } \\
\text { EVENT } \\
\text { EVENT } \\
\text { EVENT }\end{array}$ & $\begin{array}{l}0 \\
0 \\
0 \\
0 \\
0 \\
\theta\end{array}$ & $\begin{array}{r}5 \\
5 \\
15 \\
11 \\
1 \\
1\end{array}$ & $\begin{array}{l}\text { DIS= } \\
\text { DIS= } \\
\text { DIS= } \\
\text { DIS= } \\
\text { DIS= } \\
\text { DIS= }\end{array}$ & $\begin{array}{l}1.24 \\
1.19 \\
1.21 \\
1.21 \\
1.19 \\
1.24\end{array}$ & $\begin{array}{l}\text { DTIMP- } \\
\text { DTIMP= } \\
\text { DTIMP= } \\
\text { DTIMP= } \\
\text { DTIMP= } \\
\text { DTIMP= }\end{array}$ & $\begin{array}{l}0.15 \\
0.17 \\
0.24 \\
0.24 \\
0.17 \\
0.15\end{array}$ & $\begin{array}{l}34.5000 \\
34.5000 \\
34.5000 \\
34.5000 \\
34.5000 \\
34.5000\end{array}$ & $\begin{array}{l}118.4473 \\
118.4460 \\
118.4467 \\
118.5533 \\
118.5539 \\
118.5527\end{array}$ \\
\hline
\end{tabular}

TAL NUVaER OF ELEMENTAL AREAS IS 1297 MAXIMUM RAOIUS $5.77 \mathrm{kM}$

TWO DIMENSIONAL HASKEL RUPTURE MOOEL WITH STEP SLIP FUNCTION

EACH ELDUENT HAS THE SAME DISPLACENENT: $55.2 \mathrm{CM}$

TOTAL FAULTING DURATION 2.2B SECONDS

SLIP FUNCTION INCREMENTED BY DIGITIZING RATE

SOURCE EVENTS AND STNTHESIZED OUTPUT LIMITED TO 1000 POINTS

*** SOURCE EVENT (0 0 0001) ***

FAULT-STAT AZI DIP SLIP VECTOR TAKEOFF ANGLE P S INCID ANGLES

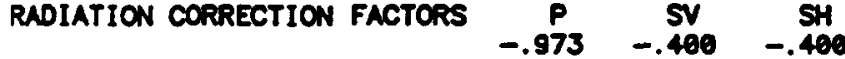

SOURCE EVENT SPLIT FOR AMLYSIS 2.00 SEC FRON ORIGIN ISS = 200

**: SOURCE EVENT (0 0 0002) **:

$\begin{array}{ccccc}\text { FAULT-STAT AZI } & \text { DIP } & \text { SLIP VECTOR } & \text { TAKEOFF ANGLE } & \text { P } 5 \text { INCID AMGLES } \\ -68.0 & 90.0 & 0.0 & 89.6 & 69.3 \\ 32.1\end{array}$

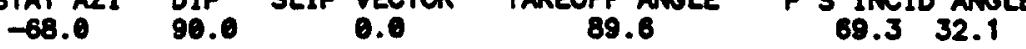


SYNTHESIS OF EVENT OO12 AT STATION 1

RADIATION CORRECTION FACTORS $\begin{array}{cccc}P & \text { SV } & \text { SH } \\ & -.694 & -.400 & -.720\end{array}$

SOURCE EVENT SPLIT FOR ANALYSIS 1.80 SEC FROM ORIGIN ISS - 179

*** SOURCE EVENT (0 0 0003) \#**

$\begin{array}{ccccc}\text { FAULT-STAT AZI } & \text { DIP } & \text { SLIP VECTOR } & \text { TAKEOFF ANGLE } & P \text { S INCID ANGLES } \\ -90.0 & 90.0 & 0.0 & 88.1 & 69.3 \quad 32.1\end{array}$ RADIATION CORRECTION FACTORS $P$ SV SH

SOURCE EVENT SPLIT FOR ANALYSIS $\overline{-.400} 1.80$ SEC FRON ORIGIN ISS $=179$

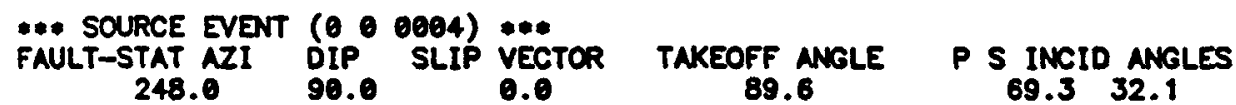

$\begin{array}{lll}\text { ELPP } & \text { ELSV } & \text { ELSH } \\ -.400 & -.400 & -.926 \\ 0.400 & 0.400 & -.932 \\ 0.400 & 0.400 & -.919 \\ 0.400 & 0.400 & -.913 \\ -.400 & -.400 & -.913 \\ -.400 & -.400 & -.919 \\ -.400 & -.400 & -.832\end{array}$




$$
\begin{array}{rrr}
-.400 & -.400 & -.940 \\
0.400 & 0.400 & -.946
\end{array}
$$

- SOURCE EVENT (0 0 0009) *\#*

$\begin{array}{ccccc}\text { FAULT-STAT AZI OIP } & \text { SLIP VECTOR } & \text { TAKEOFF ANGLE } & \text { P S INCID ANGLES } \\ 248.0 & 90.0 & 0.0 & 70.4 & 51.4 \\ \text { RADIATION CORRECTION FACTORS } & P & \text { SV } & \text { SH } & \end{array}$

$\begin{array}{rrrr}0.616 & 0.400 & -.678\end{array}$

SOURCE EVENT SPLIT FOR ANALYSIS 2.00 SEC FROM ORIGIN ISS = 200

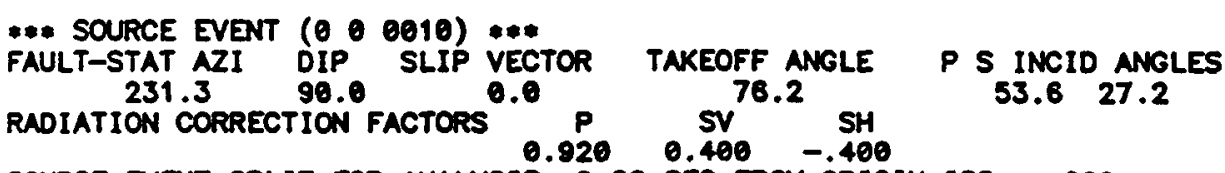


MOMENT OF MODELED EVENT $0.999966 E+25$ 


\section{Appendix A}

\section{Modeling the Main San Fernando Earthquake}

Results from independent studies are used to constrain the rupture area, fault attitude, slip vector, moment, and hypocenter of the $M=6.4,1971$ San Fernando earthquake. Then, only the stress drop, slip model, slip distribution, and rupture velocity are specified for a fault rupture model. Twenty seconds of ground acceleration are modeled, which includes all significant arrivals for the stations used.

Data

Digitized seismograms of aftershocks to the 1971 San Fernando earthquake recorded in the epicentral area are used as empirical Green's functions Hutchings, 1987). Instrument response is removed from both the strong motion recordings modeled (CIT, 1973) and the empirical Green's functions. Accelerograph sites where ground motion is modeled are listed in Table A1 along with I.D. of the associated NOAA aftershock recording site. Table A2 lists location and geology of the recording sites. Figure A1 shows the geology of the epicentral area, and locations of aftershock recording sites and sites listed in Table A1.

\section{Synthesis}

The spatial extent of modeled rupture for the San Fernando earthquake is an area that generally outlines the location of aftershocks (Allen et al., 1971) and intersects the surface along the observed surface fault rupture. It is an elliptical rupture area with major and minor axes of 25 and $10 \mathrm{~km}$, and ellipticity 0.6. The strike, dip, and slip vector are $280^{\circ}, 38^{\circ}$, and $100^{\circ}$, respectively. Figure A2 shows the fault rupture area and Figure A3 shows the location of aftershocks used as empirical Green's functions for each station. The hypocentral location of the main event has been estimated to be in the general vicinity 
area void of aftershocks (Allenet al., 1971; Hanks et al., 1974), and is shown in Figure A2. A moment of $0.86 \cdot 10^{26}$ dyne-cm is used.

Figures A4-A6 show the observed and synthesized seismograms at the three stations for the Haskel-step slip function described above along with the printout of the computer run. A rupture velocity of $2.1 \mathrm{~km} / \mathrm{s}$ was used. The absolute amplitude, energy distribution and frequency content is modeled for most components. At Pacoima Dam the absolute amplitude does not fit as well as at the other stations, but the waveforms match better. At Pacoima Dam the synthesizd records are shown for frequency bands 0.0-5.0, 0.0-10.0, and $0.0-15.0 \mathrm{~Hz}$.

\section{Conclusions}

A fairly good fit to observed seismograms in the frequency range 0.5 to $15.0 \mathrm{~Hz}$ was obtained by using a simple rupture model that might be assumed prior to the occurrence of an earthquake. The fit in absolute amplitude, frequency content, and energy distribution suggests that earthquake hazard at near-source locations can be predicted by synthesis of ground motion. Directly above the faulting, a match to amplitudes was not achieved. Here, the complexity of rupture has a much stronger effect on seismograms, and predicting ground motion is much less reliable.

Using empirical Green's functions allows one to account for many of the inhomogeneities when modeling earthquakes. However, none of the models provided a good waveform for waveform match. This is presumably due to not accurately modeling the slip function. Even so, modeled absolute amplitudes, frequency content, and energy distribution are quite good for this large earthquake. This suggests that a fairly simple rupture model can account for most of the high-frequency arrivals generated by large earthquakes. High-frequency arrivals may thus be due primarily to the constructive and destructive interference of near-source arrivals, rather than to the complexity of rupture. 
Table Al

SEISMOGRAPH RECORDING SITES

$\begin{array}{cccll}\text { STATION } & \text { LAT N } & \text { LONG W } & \text { ELEV } & \text { SITE GEOLOGY } \\ \text { ST15 } & 34-13.25 & 118-28.23 & 245 & \text { alluvium } 200 \mathrm{~m} \\ \text { ST21 } & 34-09.20 & 118-27.82 & 225 & \text { alluvium } 50 \mathrm{~m} \\ \text { ST24 } & 34-20.03 & 118-23.92 & 509 & \text { fractured diorite gneiss }\end{array}$

Table A2

MAIN EVENT ACCELEROGRAPH RECORDING SIYES

Station Name ref no Accel id NOAA stat

Holiday Inn (HOI) $241 \quad$ CO48

Bank of Cal(15250) $446 \quad$ H115 ST21

Pacoima Dam (RAC) $279 \quad$ C041




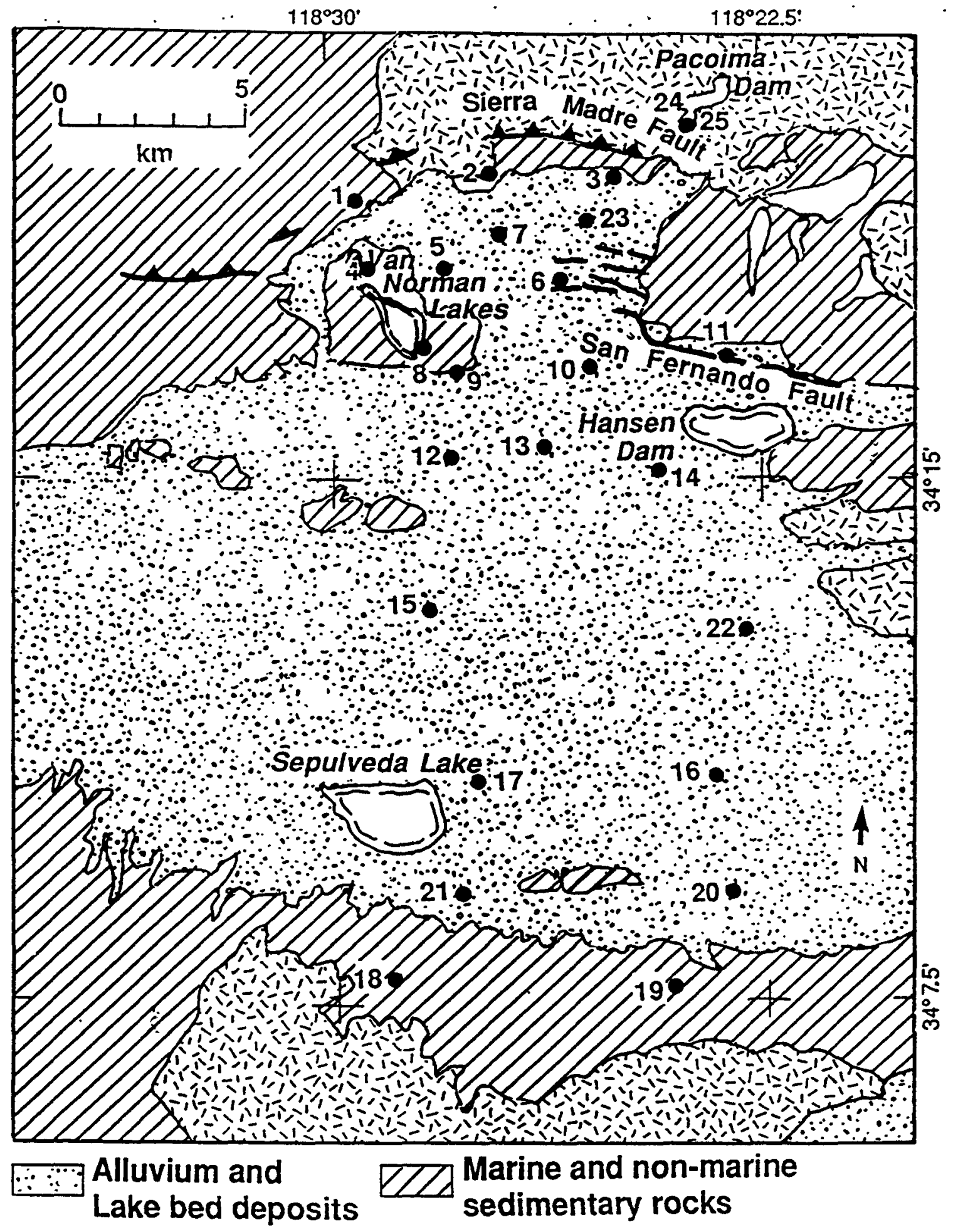

ESTGanite and diorite
gneiss

FigA1. Gcology of the study arca (modificd from Jennings and Strand [1969]). 

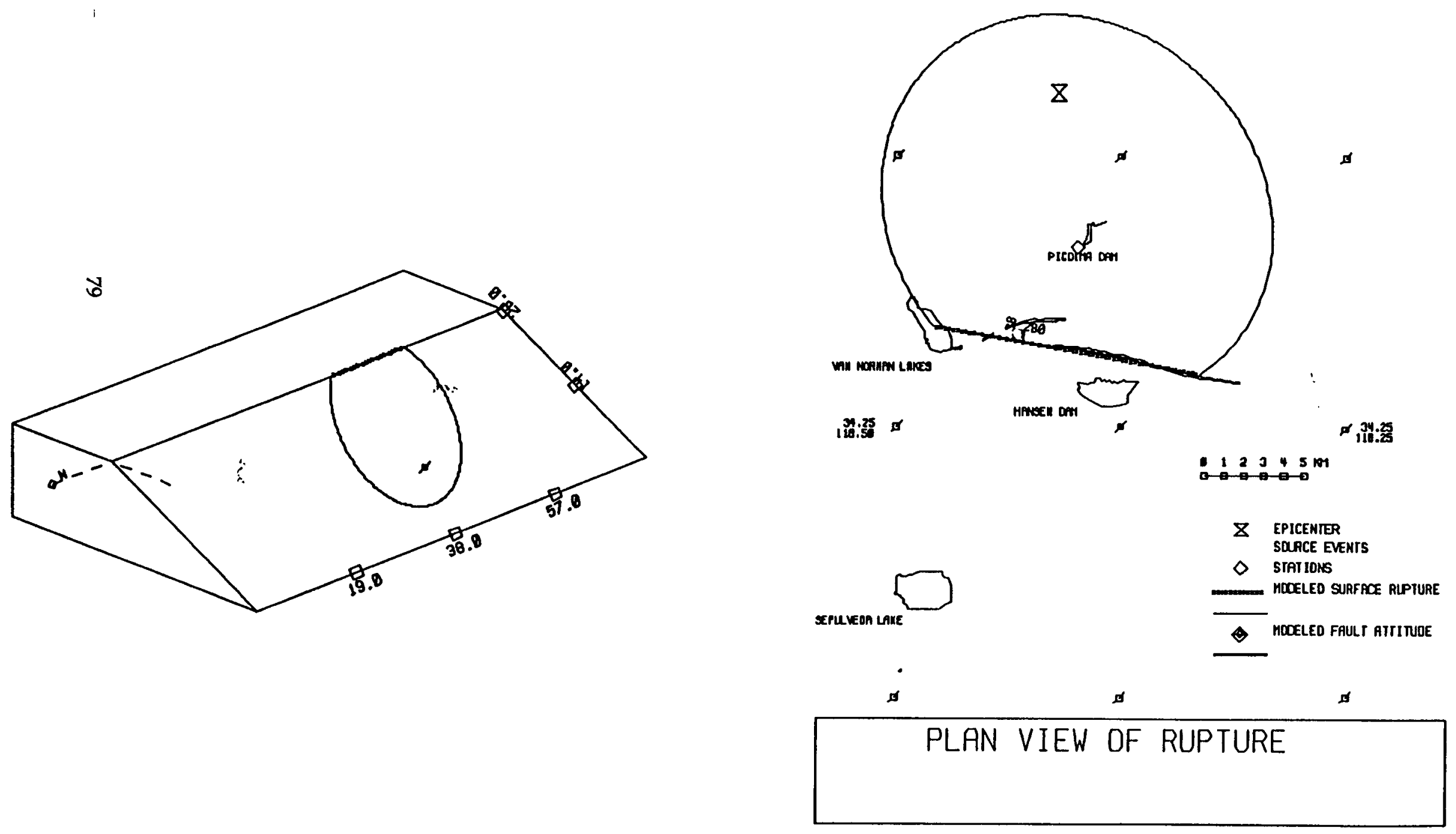


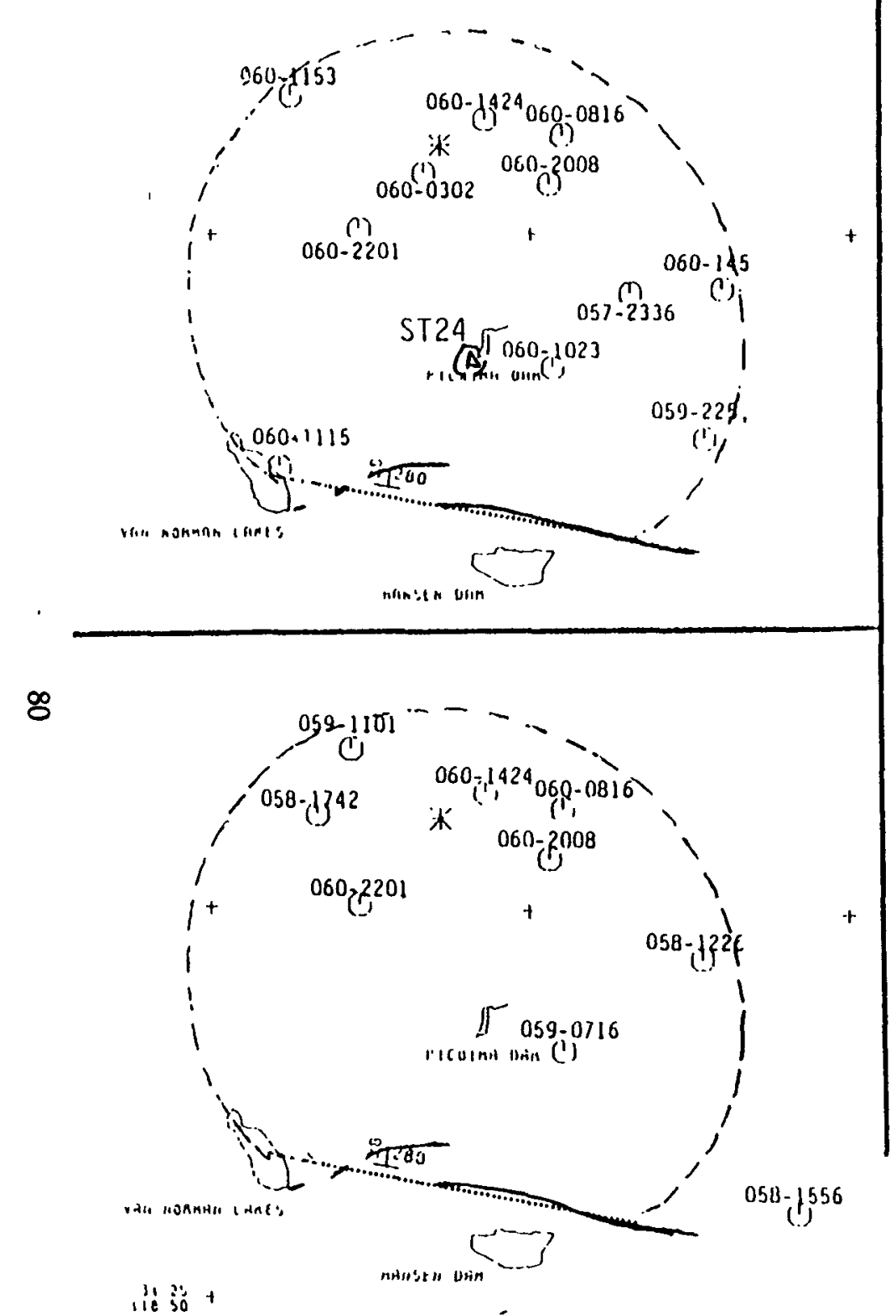

iie

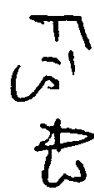

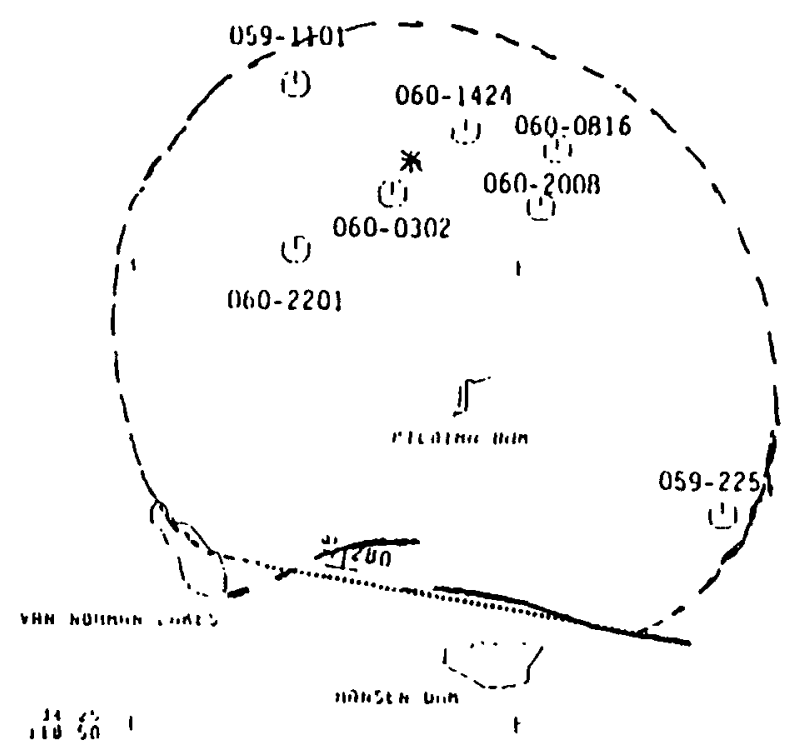

1

$$
\because: \vdots::^{\prime}: \cdots
$$

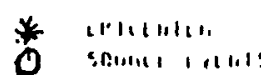

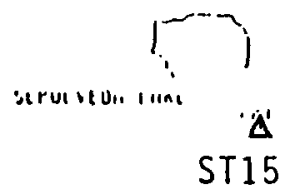

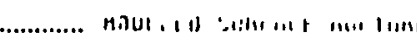

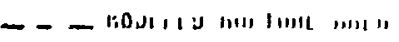

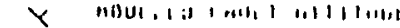

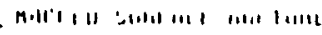
stations
Figure $A 3$ Location of source events used to obtain empirical Green's functions 


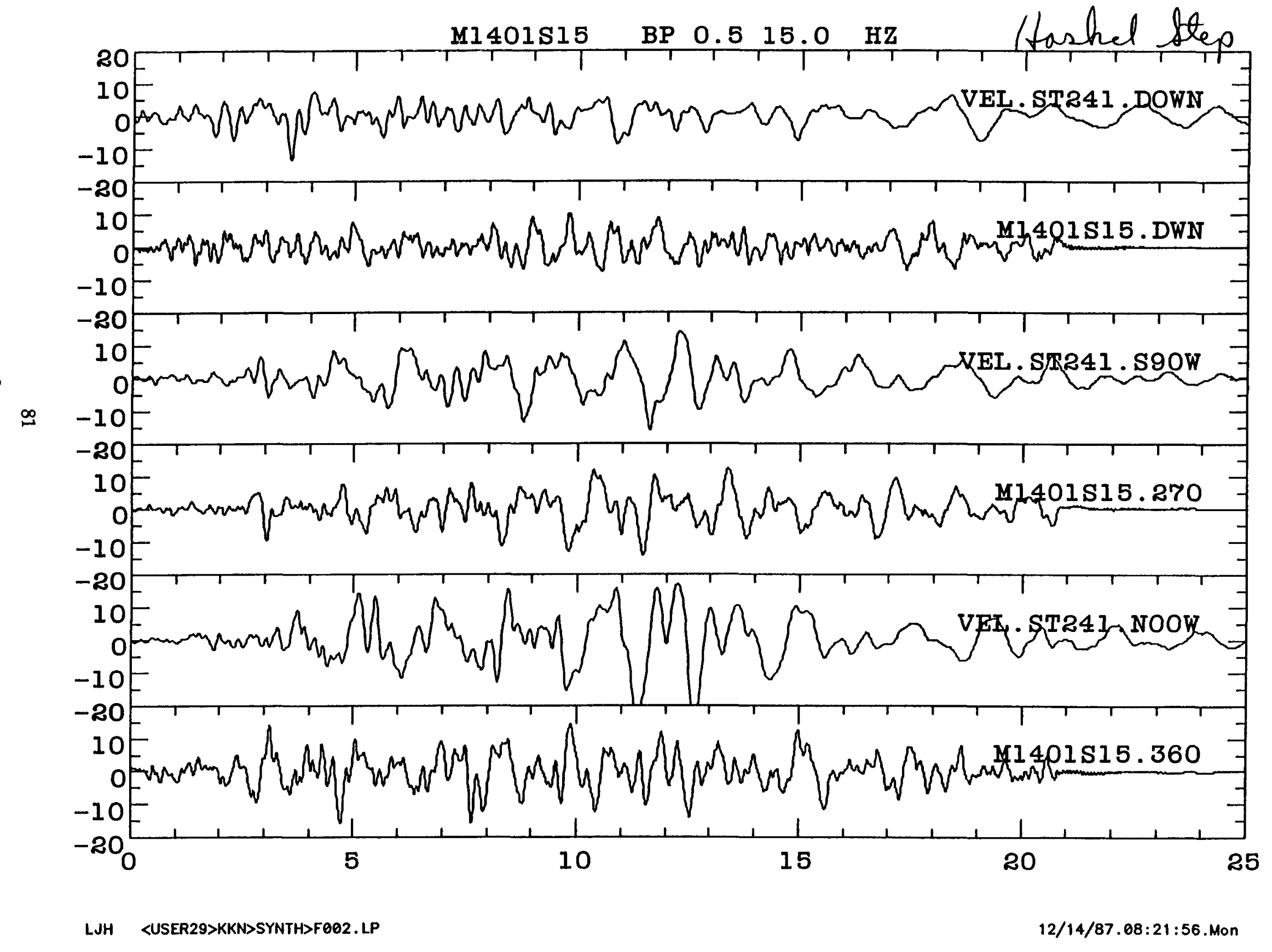


SYNTHESIS OF EVENT 1401 AT STATION 15

HYPOCENTER LOCATION

$34.4040 \quad 118.4098 \quad 10.00$

SYNTHESIZED FAULT PARAMETERS

$\begin{array}{ccccc}\text { STRIKE } & \text { DIP } & \text { SLIP VECTOR } & \text { MOMENT } & \text { ELDM AREA (KM**2) } \\ 280.0 & 38.0 & 100.0 & 0.860000 E+26 & 0.0250\end{array}$

RUPTURE VELOCITY: 2.10

P VELOCITY: VP $=3.0+H=0.20$ FOR TT CORR OF SOURCE EVENTS

ORIENTATION OF SYNTHESIZED TRACES

POSITIVE ON OUTPUT: DWN,270.0,360.0 DEGREES FROM NORTH

RADIATION CORRECTION FACTOR APPLIED FOR DIFFERENCES BETWEEN

SOURCE EVENTS AND ELEMENTS, BUT LIMITED TO -.4 TO +.4

STRESS DROP 46.5

SOURCE EVENTS

581226

581556

$58 \quad 1742$

59716

591101

$60 \quad 234$

$60 \quad 816$

$\begin{array}{ll}60 & 1424\end{array}$

602008

602201 $\begin{array}{ll}34.3600 & \text { LOCATION } \\ 118.3073\end{array}$

$34.2785 \quad 118.2698$

34.4053

34.3300

34.4263

34.4523

34.4072

34.4123

34.3917

34.3768
$118.2698 \quad 2.26$

$118.4577 \quad 9.55$

$118.3613 \quad 1.26$

$118.4450 \quad 10.84$

$118.4508 \quad 11.14$

$118.3622 \quad 13.03$

118.392310 .46

$118.3673 \quad 9.96$

118.44137 .61

\section{MOMENT}

$0.506000 E+21$

$0.875800 E+21$

$0.149050 E+22$

$0.520700 E+21$

$0.174460 E+22$

$0.200150 E+22$

0.154878E+22

$0.153650 E+22$

$0.273750 E+22$

$0.922200 E+21$
STRIKE DIP SV

276. 24. 90.

271. 50.73 .

257. 52. 68 .

218. 58. 46 .

242. 36. 83 .

238. 32. 90 .

253. 38. 98 .

268. 52. 141 .

262. 30.42 .

275. 50. -24 .

STATION: 15 SLAT= 34.2208 SLON= 118.4706

RUPTURE AREA IS ELLIPITICAL: MUJOR AXIS $25.0 \mathrm{KM}$, MINOR AXIS $10.0 \mathrm{KM}$,

ECCENTRICITY IS 0.60 ANGLE OF PERHELION OF MAJOR AXIS CLOCKWISE FROM STRIKE OF FAULT IS 285.0. FOUR POINTS ON ELLIPSE AT 90 DEG INTEVERALS FROM O DEG UP DIP: $19.0 \quad 6.95 .1 \quad 9.5 \mathrm{kM}$.

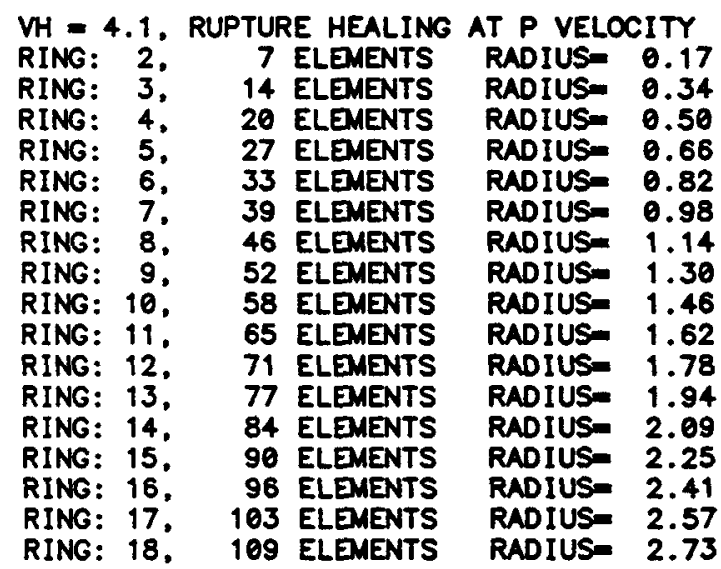


RING: 19,115 ELEMENTS

RING: 20,122 ELEMENTS

RING: $21, \quad 127$ ELEMENTS

RING: 22, 134 ELEMENTS

RING: 23, 141 ELEMENTS

RING: 24, 147 ELEMENTS

RING: 25, 153 ELEMENTS

RING: $26, \quad 159$ ELEMENTS

RING: 27,166 ELEMENTS

RING: 28, 172 ELEMENTS

RING: 29, 178 ELEMENTS

RING: 30 ,

RING: 31 ,

RING: 32 ,

RING: 33

RING: 34 ,

RING: 35 ,

RING: 36 ,

RING: 37 ,

RING: 38 ,

RING: 39 ,

RING: 40 ,

RING: 41 ,

RING: 42 .

RING: 43,

RING: 44 .

RING: 45 ,

RING: 46 .

RING: 47 ,

RING: 48,

RING: 49 ,

RING: 50 ,

RING: 51,

RING: 52 ,

RING: 53 ,

RING: 54 ,

RING: 55 ,

RING: 56 ,

RING: 57,

RING: 58,

RING: 59 .

RING: 60 ,

RING: 61 ,

RING: 62 .

RING: 63 .

RING: 64 ,

RING: 65 ,

RING: 66,

RING: 67 ,

RING: 68 ,

RING: 69 ,

RING: 70 .

RING: 71 ,

RING: 72 ,

RING: 73,

RING: 74

RING: 75

RING: 76 ,

RING: 77

185 ELEMENTS

191 ELEMENTS

197 ELEMENTS

182 ELEMENTS

175 ELEMENTS

171 ELEMENTS

168 ELEMENTS

166 ELEMENTS

165 ELEMENTS

163 ELEMENTS

163 ELEMENTS

162 ELEMENTS

161 ELEMENTS

161 ELEMENTS

161 ELEMENTS

160 ELEMENTS

160 ELEMENTS

160 ELENENTS

160 ELEMENTS

160 ELEMENTS

159 ELEMENTS

159 ELEMENTS

159 ELEMENTS

159 ELEMENTS

159 ELEMENTS

158 ELEMENTS

158 ELEMENTS

158 ELEMENTS

157 ELEMENTS

157 ELEMENTS

158 ELEMENTS

157 ELEMENTS

157 ELEMENTS

156 ELEMENTS

156 ELEMENTS

155 ELEMENTS

155 ELEMENTS

154 ELEMENTS

154 ELEMENTS

153 ELEMENTS

153 ELEMENTS

152 ELEMENTS

152 ELEMENTS

151 ELEMENTS

151 ELEMENTS

150 ELEMENTS

149 ELEMENTS

149 ELENENTS

148 ELEMENTS
RADIUS- 2.89

RADIUS- 3.05

RADIUSO 3.21

RADIUS- 3.37

RADIUS- 3.52

RADIUS- 3.68

RADIUS= $\mathbf{3 . 8 4}$

RADIUS- 4.00

RADIUS= 4.16

RADIUS- 4.32

RADIUS= 4.48

RADIUS- 4.63

RADIUS- 4.79

RADIUS= 4.95

RADIUS= 5.11

RADIUS- 5.27

RADIUS= 5.43

RADIUS= 5.59

RADIUS- 5.74

RADIUS- 5.90

RADIUS- 6.66

RADIUS- 6.22

RADIUS= 6.38

RADIUS= 6.54

RADIUS- 6.69

RADIUS= 6.85

RADIUS 7.01

RADIUS= 7.17

RADIUS= 7.33

RADIUS= 7.49

RADIUS= 7.64

RADIUSE 7.80

RADIUS- 7.96

RADIUS= 8.12

RADIUS- 8.28

RADIUS= 8.44

RADIUS- 8.59

RADIUS- 8.75

RADIUS= 8.91

RADIUS- 9.07

RADIUS= 9.23

RADIUS= 9.39

RADIUS= 9.55

RADIUS- 9.70

RADIUS= 9.86

RADIUS- 10.02

RADIUS= 10.18

RADIUS= 10.34

RADIUS- 10.49

RADIUS= 10.65

RADIUS- 10.81

RADIUS- 10.97

RADIUS= 11.13

RADIUS= 11.29

RADIUS- 11.44

RADIUS- 11.60

RADIUS- 11.76

RADIUS= 11.92

RADIUS= 12.68

RADIUS= 12.24 
RING: 79, 148 ELEMENTS RING: 80.146 ELEMENTS

RING: 81.145 ELEMENTS

RING: 82, 144 ELEMENTS

RING: 83, 144 ELEMENTS

RING: 84,143 ELEMENTS

RING: 85,142 ELEMENTS

RING: 86,141 ELEMENTS

RING: 87, 140 ELEMENTS

RING: 88, 139 ELEMENTS

RING: 89, 137 ELEMENTS

RING: 90, 136 ELEMENTS

RING: 91, 135 ELEMENTS

RING: 92, 133 ELEMENTS

RING: 93,133 ELEMENTS

RING: 94, 132 ELEMENTS

RING: 95. 130 ELEMENTS

RING: 96, 129 ELEMENTS

RING: 97, 127 ELEMENTS

RING: 98, 126 ELEMENTS

RING: 99, 123 ELENENTS

RING: 100,121 ELDMENTS

RING:101, 120 ELEMENTS

RING:102, 118 ELEMENTS

RING: $103, \quad 116$ ELEMENTS

RING:104, 92 ELENENTS

RING:105, 75 ELEMENTS

RING:106, 63 ELEMENTS

RING:107, 53 ELEMENTS

RING:108. 49 ELEMENTS

RING:109, 45 ELENENTS

RING:110, 41 ELEMENTS

RING:111, 36 ELEMENTS

RING:112, 32 ELDMENTS

RING:113, 28 ELEMENTS

RING:114, 25 ELENENTS

RING:115, 21 ELEMENTS

RING:116, 17 ELENENTS

RING:117, 13 ELEMENTS

RING:118. 9 ELEMENTS

RING:119, 5 ELEMENTS

RING: 120 .
RADIUS= 12.39

RADIUS= 12.55

RADIUS- 12.71

RADIUS- 12.87

RADIUS= 13.03

RADIUS- 13.19

RADIUS= 13.34

RADIUS- 13.50

RADIUS- 13.66

RADIUS- 13.82

RADIUSO 13.98

RADIUS= 14.14

RADIUS= 14.29

RADIUSO 14.45

RADIUS- 14.61

RADIUS- 14.77

RADIUSO 14.93

RADIUS= 15.08

RADIUS- 15.24

RADIUS- 15.40

RADIUS- 15.56

RADIUS= 15.72

RADIUS- 15.88

RADIUS- 16.03

RADIUSm 16.19

RADIUSA 16.35

RADIUS= 16.51

RADIUS= 16.67

RADIUSO 16.83

RADIUS- 18.98

RADIUSO 17.14

RADIUS 17.30

RADIUSE 17.46

RADIUS- 17.62

RADIUSm 17.77

RADIUS= 17.93

RADIUS- 18.09

RADIUSE 18.25

RADIUS- 18.41

RADIUS 18.57

RADIUS- 18.72

RADIUS- 18.88

TOTAL NUMBER OF ELEMENTAL AREAS IS 14423

TOTAL FAULTING DURATION: 11.50

***** ELEMENTS ****

HYPOCENTER:

RISTM $=3.61 \quad$ DISP= $128.74 \mathrm{CM}$

SYNTHESIS IS BY CONVOLUTION OF SOURCE EVENTS WITH SLIP FUNCTION,

DECONVOLVED WITH STEP FUNCTION. EACH SOURCE EVENT IS SPLIT BETWEEN P AND $S$.

AND COMPUTATION IS DONE IN FREQUENCY DOMIN. FFT FOR EACH SOURCE EVENT ONCE.

RAMP DISPLACEMENT FUNCTION - MODIFIED KOSTROV

SLIP AMPLITUDE AND RISE TIME CONSTRAINED BY SELF-SIMILAR KOSTROV SLIP

ELEMENT DISPLACEMENT (LEFT TO RIGHT)

FIRST 160 ELEMENTS

$\begin{array}{llllllll}128.741 & 129.044 & 128.204 & 127.725 & 128.099 & 128.962 & 129.615 & 129.611\end{array}$ 


\begin{tabular}{|c|c|c|c|c|c|c|c|}
\hline $\begin{array}{l}130.046 \\
128.327 \\
129.789 \\
126.412 \\
31.285 \\
127.449 \\
125.669 \\
132.245 \\
131.797 \\
123.959 \\
126.481 \\
33.312 \\
133.424 \\
125.971 \\
22.045 \\
30.133 \\
33.970 \\
34.268 \\
12.267\end{array}$ & $\begin{array}{l}129.431 \\
129.269 \\
128.889 \\
127.325 \\
131.139 \\
126.545 \\
126.623 \\
132.260 \\
130.929 \\
123.467 \\
127.631 \\
133.243 \\
133.103 \\
124.762 \\
122.529 \\
131.322 \\
133.535 \\
133.989 \\
125.345\end{array}$ & $\begin{array}{l}128.573 \\
130.059 \\
127.993 \\
128.370 \\
131.756 \\
125.603 \\
127.667 \\
132.070 \\
130.097 \\
123.153 \\
128.874 \\
133.000 \\
132.486 \\
123.838 \\
123.231 \\
132.481 \\
133.197 \\
133.136 \\
124.226\end{array}$ & $\begin{array}{l}127.702 \\
130.482 \\
127.010 \\
129.403 \\
131.509 \\
124.968 \\
128.820 \\
131.934 \\
129.061 \\
123.093 \\
130.068 \\
132.674 \\
131.580 \\
122.988 \\
124.139 \\
133.362 \\
133.167 \\
132.472 \\
123.108\end{array}$ & $\begin{array}{l}127.013 \\
130.545 \\
126.185 \\
130.405 \\
131.055 \\
124.484 \\
129.896 \\
131.809 \\
127.811 \\
123.318 \\
131.312 \\
132.533 \\
130.626 \\
122.413 \\
125.239 \\
133.927 \\
133.169 \\
131.270 \\
122.262\end{array}$ & $\begin{array}{l}126.667 \\
130.390 \\
125.685 \\
130.947 \\
130.374 \\
124.349 \\
130.934 \\
132.565 \\
126.787 \\
123.796 \\
132.206 \\
132.497 \\
129.448 \\
121.974 \\
126.373 \\
134.376 \\
134.164 \\
130.289 \\
121.481\end{array}$ & $\begin{array}{l}126.832 \\
130.998 \\
125.548 \\
131.315 \\
129.486 \\
124.588 \\
131.593 \\
132.420 \\
125.735 \\
124.515 \\
132.633 \\
133.359 \\
128.288 \\
121.765 \\
127.616 \\
134.701 \\
134.420 \\
129.078 \\
120.954\end{array}$ & $\begin{array}{l}127.442 \\
130.506 \\
125.802 \\
131.357 \\
128.577 \\
124.955 \\
132.123 \\
132.224 \\
124.737 \\
125.367 \\
133.122 \\
133.484 \\
127.008 \\
121.789 \\
128.941 \\
134.334 \\
134.481 \\
127.697 \\
120.543 \\
\text { SECONDS }\end{array}$ \\
\hline
\end{tabular}

*** SOURCE EVENT (058 1226) *.*

FAULT-STAT AZI DIP SLIP VECTOR
-51.8
RADIATION CORRECTION FACTORS

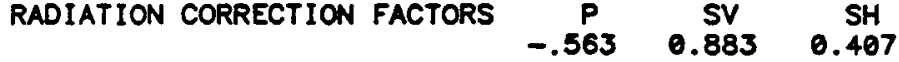

SOURCE EVENT SPLIT FOR ANALYSIS 6.50 SEC FROM ORIGIN ISS = 396

*** SOURCE EVENT (058 1556) ***

$\begin{array}{ccccc}\text { FAULT-STAT AZI DIP } & \text { SLIP VECTOR TAKEOFF ANGLE } & \text { P S INCID ANGLES } \\ -20.1 & 50.0 & 73.0 & 64.6 & 51.4 \\ 26.4\end{array}$

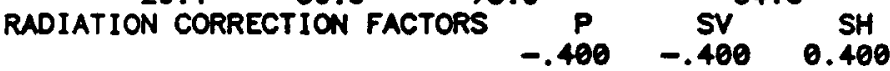

SOURCE EVENT SPLIT FOR ANALYSIS 7.00 SEC FROM ORIGIN ISS = 543

*** SOURCE EVENT (058 1742) ***

FAULT-STAT AZI DIP SLIP VECTOR TAKEOFF ANGLE PS INCID ANGLES

RADIATION CORRECTION FACTORS $P$ P SV 87.0 SH

SOURCE EVENT SPLIT FOR ANALYSIS 8.00 SEC FROM ORIGIN ISS $=400$

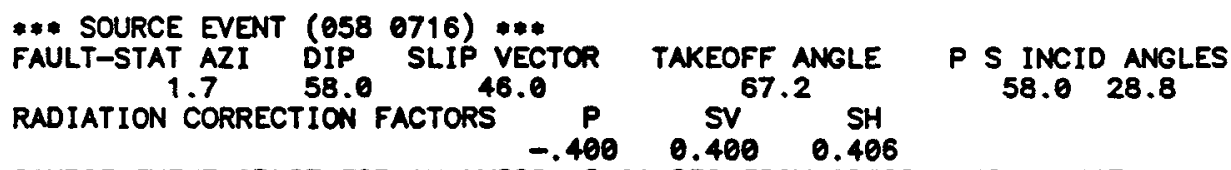

SOURCE EVENT SPLIT FOR ANALYSIS 8.00 SEC FROM ORIGIN ISS $=847$

*** SOURCE EVENT (058 1101) ***

$\begin{array}{ccccc}\text { FAULT-STAT AZI } & \text { DIP } & \text { SLIP VECTOR } & \text { TAKEOFF ANGLE } & \text { P S INCID ANGLES } \\ -56.1 & 36.0 & 83.0 & 85.5 & 34.8 \\ 18.9\end{array}$

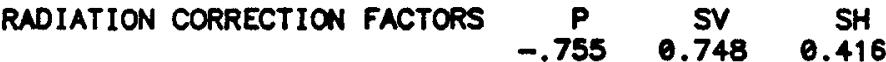


SOURCE EVENT SPLIT FOR ANALYSIS 8.50 SEC FROM ORIGIN ISS = 550

*** SOURCE EVENT (058 0234) ***

FAULT-STAT AZI DIP SLIP VECTOR -53.9
-52.0

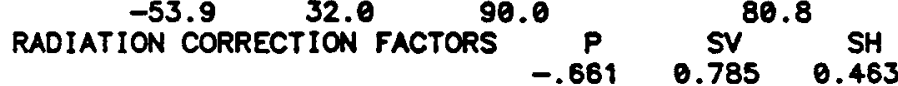

TAKEOFF ANGLE 80.8

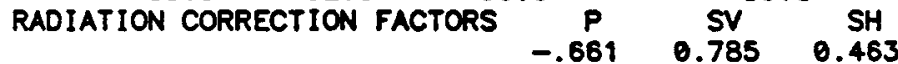

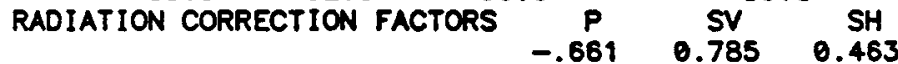

SOURCE EVENT SPLIT FOR ANALYSIS 9.00 SEC FROM ORIGIN ISS = 500
*** SOURCE EVENT (058 0816) ***
FAULT-STAT AZI DIP SLIP VECTOR TAKEOFF ANGLE $P$ S INCID ANGLES

-47.2
RADIATION CORRECTION FACTORS

$$
\begin{array}{lll}
-.529 & 0.661 & 0.486
\end{array}
$$

SOURCE EVENT SPLIT FOR ANALYSIS 7.00 SEC FROM ORIGIN ISS = 396

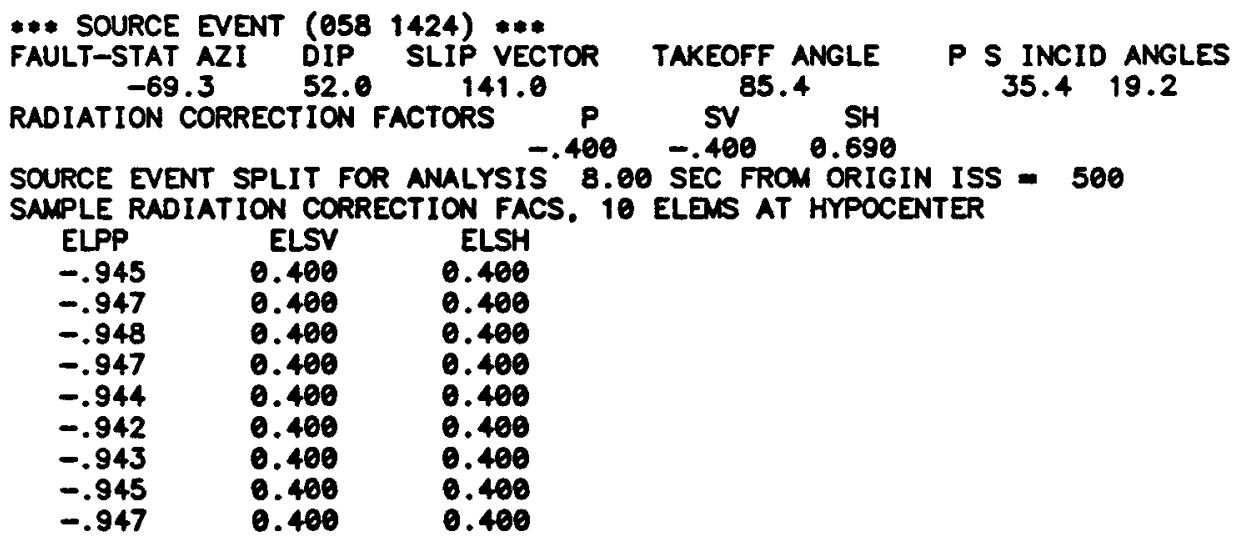

$\begin{array}{lll}\text { ELPP } & \text { ELSV } & \text { ELSH } \\ -.945 & 0.400 & 0.400 \\ -.947 & 0.400 & 0.400 \\ -.948 & 0.400 & 0.400 \\ -.947 & 0.400 & 0.400 \\ -.944 & 0.400 & 0.400 \\ -.942 & 0.400 & 0.400 \\ -.943 & 0.400 & 0.400 \\ -.945 & 0.400 & 0.400 \\ -.947 & 0.400 & 0.400\end{array}$

*** SOURCE EVENT (058 2008) **: FAULT-STAT AZI DIP SLIP VECTOR TAKEOFF AMGLE P S INCID ANGLES

$42.0 \mathrm{P} S \mathrm{SV}^{86.7} \mathrm{SH}$

36.319 .7

RADIATION CORRECTION FACTORS

$\begin{array}{lll}-.810 & 0.888 & 0.400\end{array}$

SOURCE EVENT SPLIT FOR ANALYSIS 8.00 SEC FROM ORIGIN ISS = 500

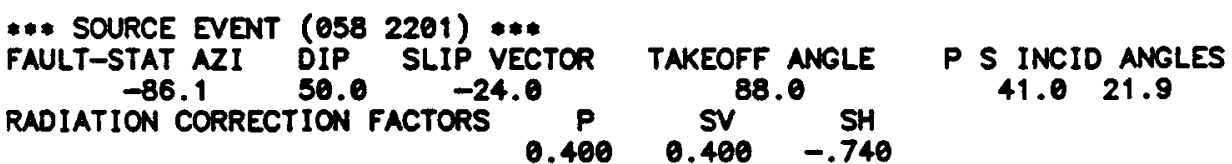

SOURCE EVENT SPLIT FOR ANALYSIS 5.50 SEC FROM ORIGIN ISS = 450

MOMENT OF ACTUAL EVENT $0.860000 E+28$

MOMENT OF MODELED EVENT $0.119901 E+27$

SLAT= 34.2208 SLONE 118.4706 DIS= 19.23 AZINY= 207.2

$0.29-0.29-1.010 .72203$ LOOP

$\begin{array}{llllll}0.26 & 0.33 & 1.26 & -1.83 & 202 & \text { LOOP }\end{array}$

$I A=64$ AB $\quad 2.967$ ADIS= 2.592 


\section{Harhersty}

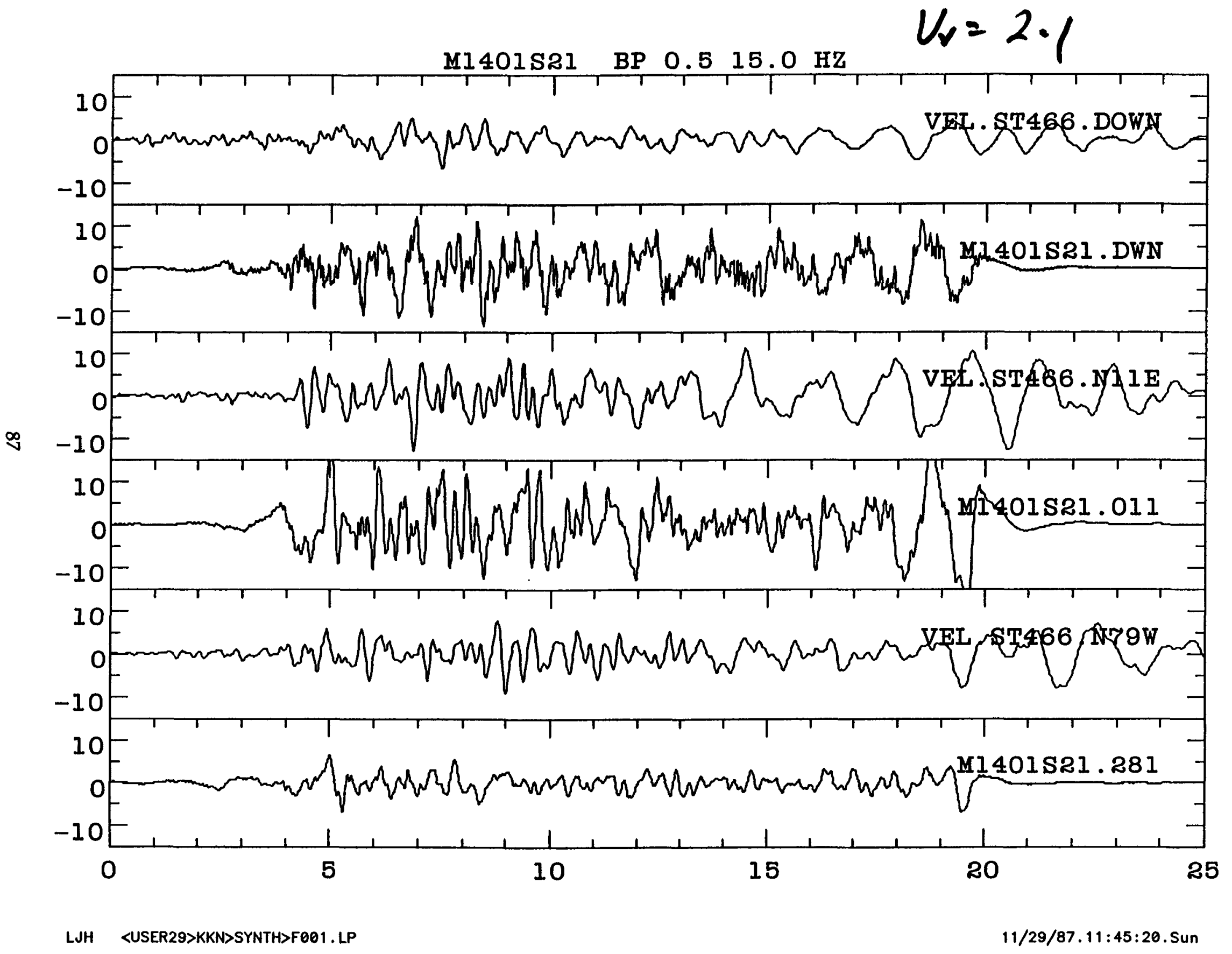




\section{SYNTHESIS OF EVENT 1401 AT STATION 21}

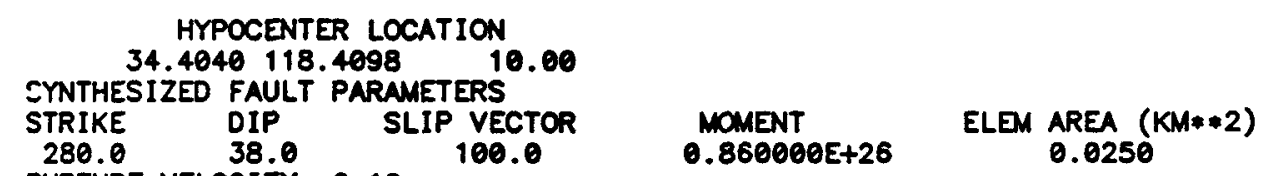

RUPTURE VELOCITY: 2.10

$P$ VELOCITY: VP $=3.0+H * 0.20$ FOR TT CORR OF SOURCE EVENTS

ORIENTATION OF SYNTHESIZED TRACES

POSITIVE ON OUTPUT: DWN, 11.0.101.0 DEGREES FROM NORTH

RADIATION CORRECTION FACTOR APPLIED FOR DIFFERENCES BETWEEN

SOURCE EVENTS AND ELEMENTS, BUT LIMITED TO -.4 TO +.4

STRESS DROP 46.5

\begin{tabular}{ccccccccr} 
SOURCE EVENTS & & LOCATION & & MOMENT & STRIKE & DIP & \multicolumn{1}{c}{ SV } \\
59 & 1101 & 34.4263 & 118.4450 & 10.84 & $0.174460 E+22$ & 242. & 36. & 83. \\
59 & 2251 & 34.3078 & 118.3075 & 10.09 & $0.126150 E+22$ & 326. & 66. & 147. \\
60 & 816 & 34.4072 & 118.3622 & 13.03 & $0.154870 E+22$ & 253. & 38. & 90. \\
60 & 1424 & 34.4123 & 118.3923 & 10.46 & $0.153650 E+22$ & 268. & 52. & 141. \\
60 & 2008 & 34.3917 & 118.3673 & 9.96 & $0.273750 E+22$ & 262. & 30. & 42. \\
60 & 2201 & 34.3768 & 118.4413 & 7.61 & $0.922200 E+21$ & 275. & 50. & -24. \\
61 & 302 & 34.3950 & 118.4160 & 7.89 & $0.377390 E+22$ & 300. & 70. & 142.
\end{tabular}

STATION: 21 SLAT= 34.1533 SLON 118.4636

RUPTURE AREA IS ELLIPITICAL: MAJOR AXIS $25.0 \mathrm{KM}$. MINOR AXIS $10.0 \mathrm{KM}$.

ECCENTRICITY IS 0.60 ANGLE OF PERHELION OF MAJOR AXIS CLOCKWISE FROM STRIKE OF FAULT IS 285.0. FOUR POINTS ON ELLIPSE AT 90 DEG INTEVERALS FROM $O$ DEG UP DIP: $19.06 .95 .1 \quad 9.5 \mathrm{kM}$.

VH $=4.1$, RUPTURE HEALING AT P VELOCITY

RING: 2 , 7 ELENENTS RADIUS- 0.17

RING: $3, \quad 14$ ELEMENTS RADIUS= 0.34

RING: 4,20 ELENENTS RADIUS- 0.50

RING: 5,27 ELEMENTS RADIUS= 0.66

RING: $6, \quad 33$ ELEMENTS RADIUS= 0.82

RING: $7, \quad 39$ ELEMENTS RADIUS- 0.98

RING: 8,46 ELEMENTS RADIUS- 1.14

RING: 9; 52 ELEMENTS RADIUS= 1.30

RING: $10, \quad 58$ ELEMENTS RADIUS- 1.46

RING: 11. 65 ELEMENTS RADIUS= 1.62

RING: $12, \quad 71$ ELEMENTS RADIUS- 1.78

RING: 13,77 ELEMENTS RADIUS- 1.94

RING: 14, 84 ELEMENTS RADIUS- 2.09

RING: 15,90 ELEMENTS RADIUS= 2.25

RING: 16, 96 ELEMENTS RADIUS- 2.41

RING: 17,103 ELEMENTS RADIUS= 2.57

RING: 18, 109 ELEMENTS RADIUS- 2.73

RING: 19,115 ELEMENTS RADIUS= 2.89

RING: 20,122 ELENENTS RADIUS- 3.05

RING: 21, 127 ELEMENTS RADIUS- 3.21 
RING: 22, 134 ELEMENTS

RING: 23,141 ELEMENTS

RING: 24, 147 ELENENTS

RING: 25, 153 ELEMENTS

I.ING: 26, 159 ELEMENTS

RING: 27, 166 ELEMENTS

RING: 28, 172 ELENENTS

RING: 29; 178 ELENENTS

RING: 30.185 ELENENTS

RING: 31 .

191 ELENENTS

RING: 32, 197 ELEMENTS

RING: 33, 182 ELEMENTS

RING: $34, \quad 175$ ELENENTS

RING: 35, 171 ELEMENTS

RING: 36, 168 ELEMENTS

RING: $37, \quad 166$ ELENENTS

RING: 38 ,

RING: 39 ,

165 ELEMENTS

163 ELENENTS

RING: 40,163 ELEMENTS

RING: 41, 162 ELEMENTS

RING: 42, 161 ELEMENTS

RING: 43, 161 ELEMENTS

RING: 44, 161 ELEMENTS

RING: 45, 160 ELENENTS

RING: 46, 160 ELEMENTS

RING: 47,160 ELEMENTS

RING: 48, 160 ELENENTS

RING: 49, 160 ELEMENTS

RING: 50,159 ELENENTS

RING: $51, \quad 159$ ELEMENTS

RING: 52. 159 ELENENTS

RING: 53, 159 ELENENTS

RING: 54,159 ELEMENTS

RING: 55, 158 ELEMENTS

RING: 56, 158 ELEMENTS

RING: 57, 158 ELEMENTS

RING: 58,157 ELEMENTS

RING: 59, 157 ELEMENTS

RING: 60,

RING: 61 .

RING: 62,

RING: 63 ,

RING: 64 ,

RING: 65 ,

RING: 66 ,

RING: 67 ,

RING: 68 ,

RING: 69 ,

RING: 70 ,

RING: 71 ,

RING: 72,

RING: 73 ,

RING: 74 ,

RING: 75,

RING: 76,

RING: 77,

RING: 78,

RING: 79 ,

158 ELEMENTS

157 ELEMENTS

157 ELENENTS

156 ELEMENTS

156 ELEMENTS

155 ELEMENTS

155 ELENENTS

154 ELEMENTS

154 ELEMENTS

153 ELEMENTS

153 ELEMENTS

152 ELEMENTS

152 ELEMENTS

151 ELEMENTS

151 ELENENTS

150 ELENENTS

149 ELEMENTS

149 ELENENTS

RING: 80 ,

148 ELEMENTS

148 ELENENTS

RING: 81 ,
RADIUS= 3.37
RADIUS= 3.52
RADIUS- 3.68
RADIUS= 3.84
RADIUS= 4.00
RADIUS= 4.16
RADIUS= 4.32
RADIUS- 4.48
RADIUS- 4.63
RADIUS- 4.79
RADIUS- 4.95
RADIUS- $\mathbf{5 . 1 1}$
RADIUS- 5.27
RADIUS- 5.43
RADIUS= $\mathbf{5 . 5 9}$
RADIUS= $\mathbf{5 . 7 4}$
RADIUS- $\mathbf{5 . 9 0}$
RADIUS- 6.06
RADIUS- 6.22
RADIUS- 6.38
RADIUS- 6.54
RADIUS= 6.69
RADIUS- 6.85
RADIUS- 7.01
RADIUS 7.17
RADIUS= 7.33
RADIUS= 7.49
RADIUS= 7.64
RADIUSE 7.80
RADIUSE 7.96
RADIUS- 8.12
RADIUSE 8.28
RADIUS= 8.44
RADIUS- $\mathbf{8 . 5 9}$
RADIUS= 8.75
RADIUS= $\mathbf{8 . 9 1}$
RADIUS= 9.07
RADIUS- 9.23
RADIUS= 9.39
RADIUS- 9.55
RADIUS- 9.70
RADIUS- 9.86
RADIUS- 10.02
RADIUS- 10.18
RADIUS- 10.34
RADIUS- 10.49
RADIUS- 10.65
RADIUS- 10.81
RADIUS- 10.97
RADIUS- 11.13
RADIUS= 11.29
RADIUS= 11.44
RADIUS- 11.60
RADIUS- 11.76
RADIUS= 11.92
RADIUS- 12.08
RADIUS- 12.24
RADIUS= 12.39
RADIUS- 12.55

145 ELEMENTS RADIUS- 12.71 
TOTAL NUMBER OF ELEMENTAL AREAS IS 14423 TOTAL FAULTING DURATION: 11.50

***** ELEMENTS ****
MAXIMUM RADIUS $18.96 \mathrm{KM}$

\author{
DISPm $128.74 \mathrm{CM}$
}

SYNTHESIS IS BY CONVOLUTION OF SOURCE EVENTS WITH SLIP FUNCTION.

DECONVOLVED WITH STEP FUNCTION. EACH SOURCE EVENT IS SPLIT BETWEEN $P$ AND $S$, AND COMPUTATION IS DONE IN FRECUENCY DOMAIN. FFT FOR EACH SOURCE EVENT ONCE. RAMP DISPLACENENT FUNCTION - MODIFIED KOSTRON

SLIP AMPLITUDE AND RISE TIME CONSTRAINED BY SELF-SIMILAR KOSTROV SLIP ELEMENT DISPLACEMENT (LEFT TO RIGHT)

FIRST 160 ELEMENTS

$\begin{array}{lllllllll}128.741 & 129.044 & 128.204 & 127.725 & 128.099 & 128.962 & 129.615 & 129.611 \\ 130.046 & 129.431 & 128.573 & 127.702 & 127.013 & 126.667 & 126.832 & 127.442 \\ 128.327 & 129.269 & 130.059 & 130.482 & 130.545 & 130.390 & 130.908 & 130.506 \\ 129.789 & 128.889 & 127.993 & 127.010 & 126.185 & 125.685 & 125.548 & 125.802\end{array}$




$\begin{array}{lllllllll}126.412 & 127.325 & 128.370 & 129.403 & 130.405 & 130.947 & 131.315 & 131.357 \\ 131.285 & 131.139 & 131.756 & 131.509 & 131.055 & 130.374 & 129.486 & 128.577 \\ 127.449 & 126.545 & 125.603 & 124.908 & 124.484 & 124.349 & 124.508 & 124.955 \\ 125.669 & 126.623 & 127.667 & 128.820 & 129.890 & 130.934 & 131.593 & 132.123 \\ 132.245 & 132.260 & 132.070 & 131.934 & 131.809 & 132.565 & 132.420 & 132.224 \\ 131.797 & 130.929 & 130.007 & 129.061 & 127.811 & 126.787 & 125.735 & 124.737 \\ 123.959 & 123.467 & 123.153 & 123.093 & 123.318 & 123.796 & 124.515 & 125.367 \\ 126.481 & 127.631 & 128.874 & 130.008 & 131.312 & 132.206 & 132.633 & 133.122 \\ 133.312 & 133.243 & 133.000 & 132.674 & 132.533 & 132.497 & 133.359 & 133.484 \\ 133.424 & 133.103 & 132.486 & 131.580 & 130.626 & 129.448 & 128.288 & 127.008 \\ 125.971 & 124.762 & 123.838 & 122.988 & 122.413 & 121.974 & 121.765 & 121.789 \\ 122.045 & 122.529 & 123.231 & 124.139 & 125.239 & 126.373 & 127.616 & 128.941 \\ 130.133 & 131.322 & 132.481 & 133.362 & 133.927 & 134.376 & 134.701 & 134.334 \\ 133.970 & 133.535 & 133.197 & 133.107 & 133.169 & 134.164 & 134.420 & 134.481 \\ 134.268 & 133.989 & 133.136 & 132.472 & 131.270 & 130.289 & 129.078 & 127.697 \\ 126.573 & 125.345 & 124.226 & 123.108 & 122.262 & 121.481 & 120.954 & 120.543 \\ \text { SPECTRAL DIMENSION IS 4096 POINTS. IF TRACES ARE GREATER THAN 35 SECONDS } \\ \text { YOU GET WRAP-AROUND IN MAIN SEISIOGRAM } & & & & \end{array}$

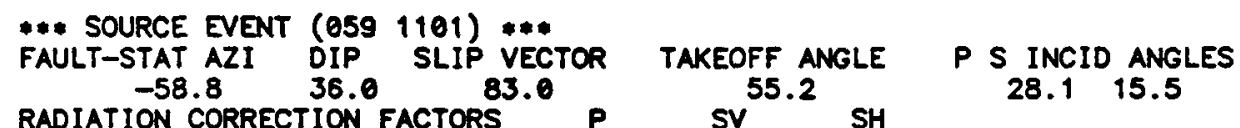

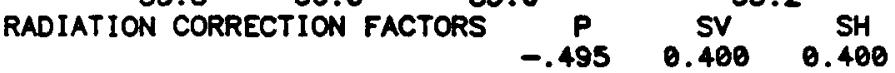

SOURCE EVENT SPLIT FOR ANALYSIS 9.50 SEC FROM ORIGIN ISS = 651

** SOURCE EVENT (059 2251) ***

$\begin{array}{ccccc}\text { FAULT-STAT AZI DIP } & \text { SLIP VECTOR } & \text { TAKEOFF ANGLE } & \text { P S INCID ANGLES } \\ -106.0 & 66.0 & 147.0 & 84.6 & 36.0 \\ \text { RADIATION CORRECTION FACTORS } & P & \text { SY } & \text { SH } & \end{array}$

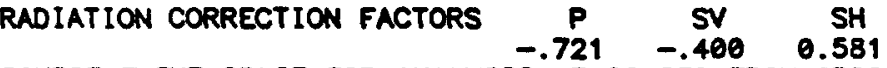

SOURCE EVENT SPLIT FOR ANALYSIS 7.00 SEC FROM ORIGIN ISS = 382

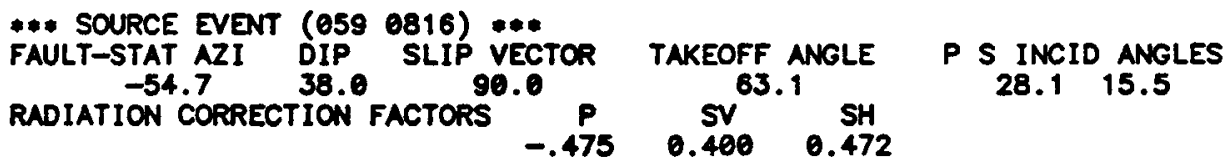

SOURCE EVENT SPLIT FOR ANALYSIS 8.50 SEC FRON ORIGIN ISS = 535

*** SOURCE EVENT (059 1424) ***

FAULT-STAT AZI DIP SLIP VECTOR TAKEOFF ANGLE P S INCID ANGLES

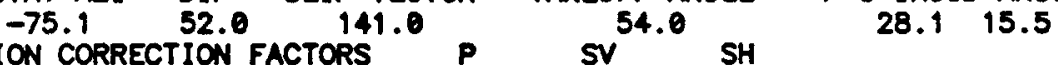

RADIATION CORRECTION FACTORS $P$ SV

SOURCE EVENT SPLIT FOR ANALYSIS 8.00 SEC FROM ORIGIN ISS = 388

SANPLE RADIATION CORRECTION FACS, 10 ELENS AT HYPOCENTER

$\begin{array}{lll}\text { ELPP } & \text { ELSV } & \text { ELSH } \\ -.479 & 0.400 & 0.400 \\ -.474 & 0.400 & 0.400 \\ -.483 & 0.400 & 0.400 \\ -.489 & 0.400 & 0.400 \\ -.487 & 0.400 & 0.400 \\ -.480 & 0.400 & 0.400 \\ -.472 & 0.400 & 0.400 \\ -.469 & 0.400 & 0.400\end{array}$




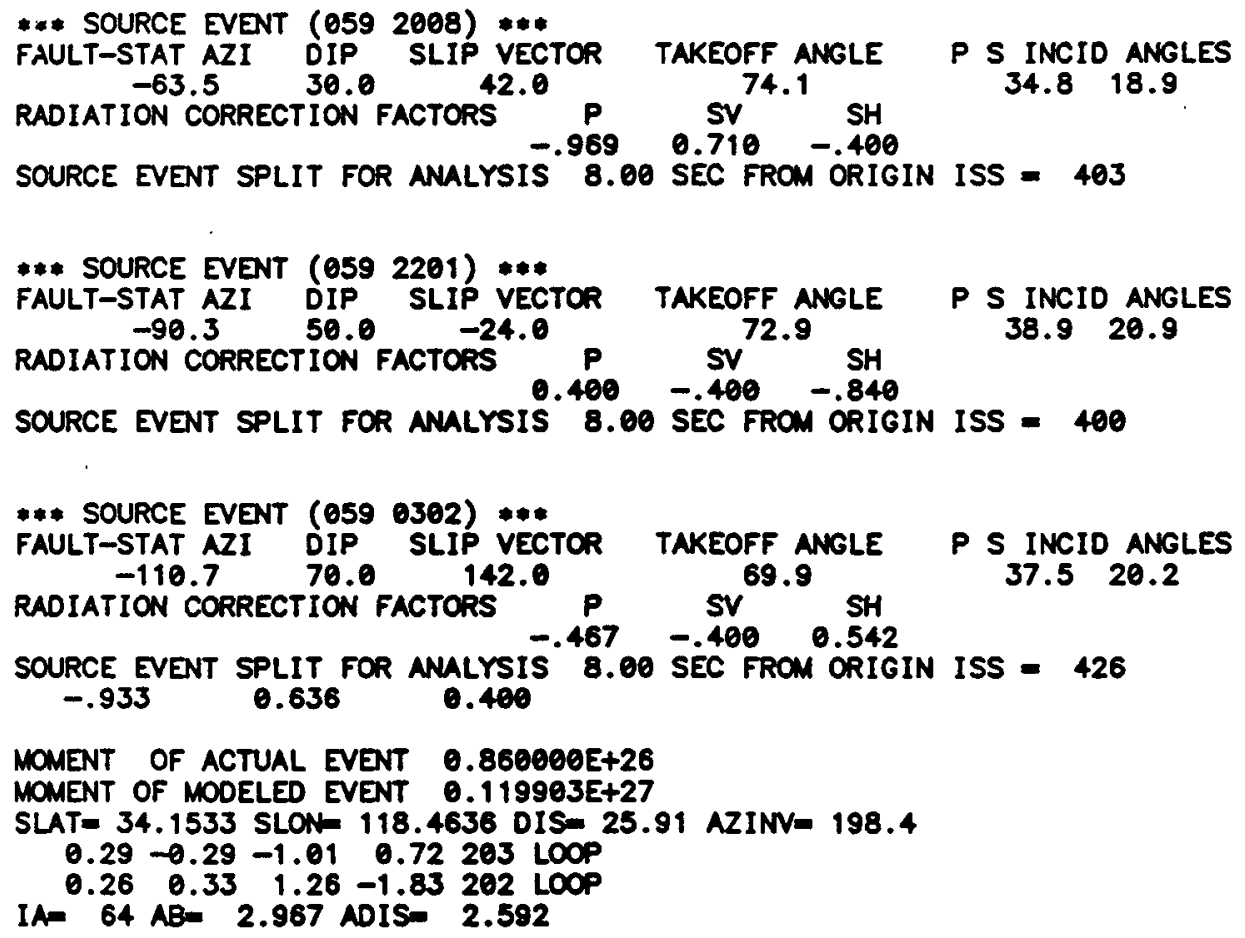




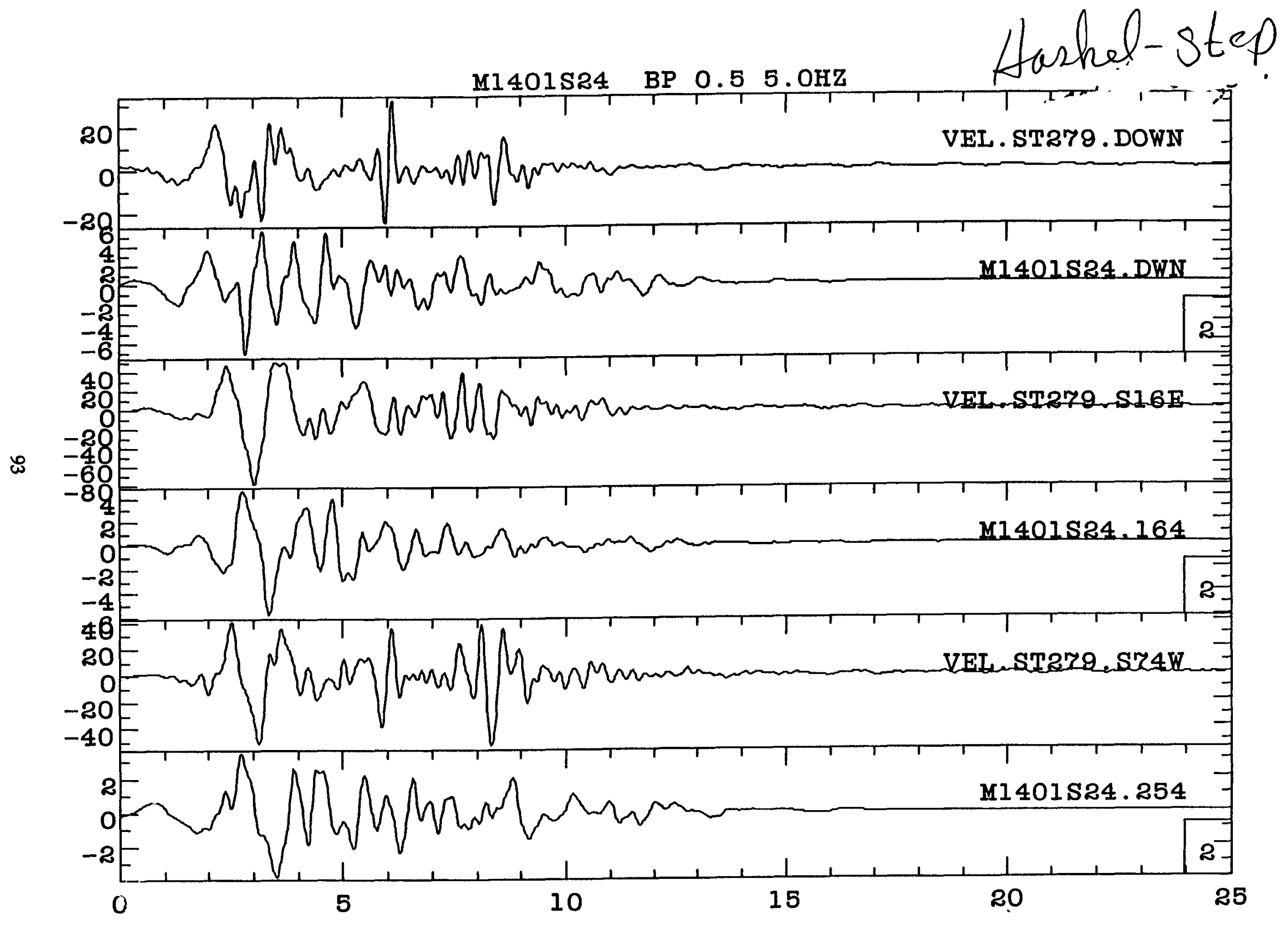




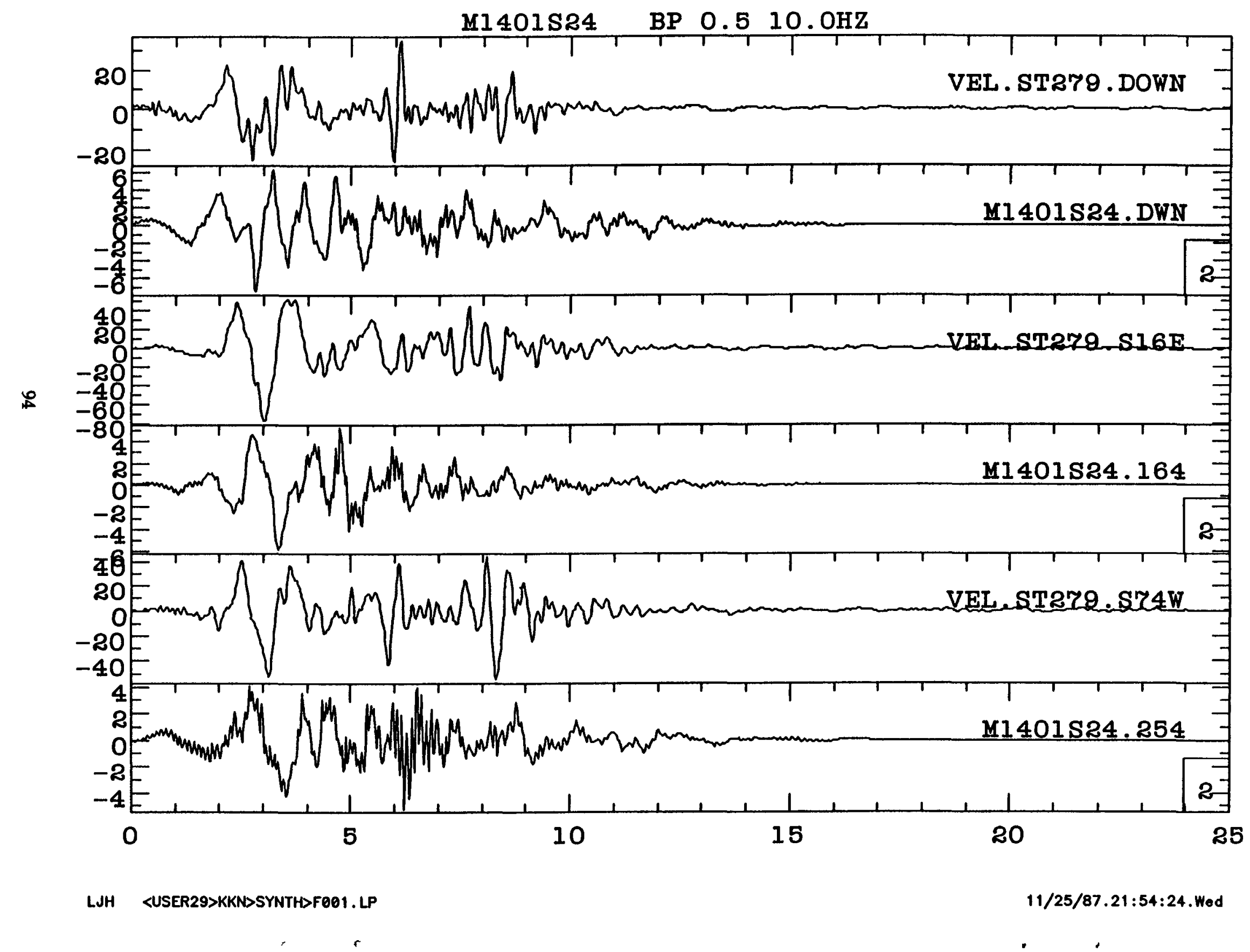




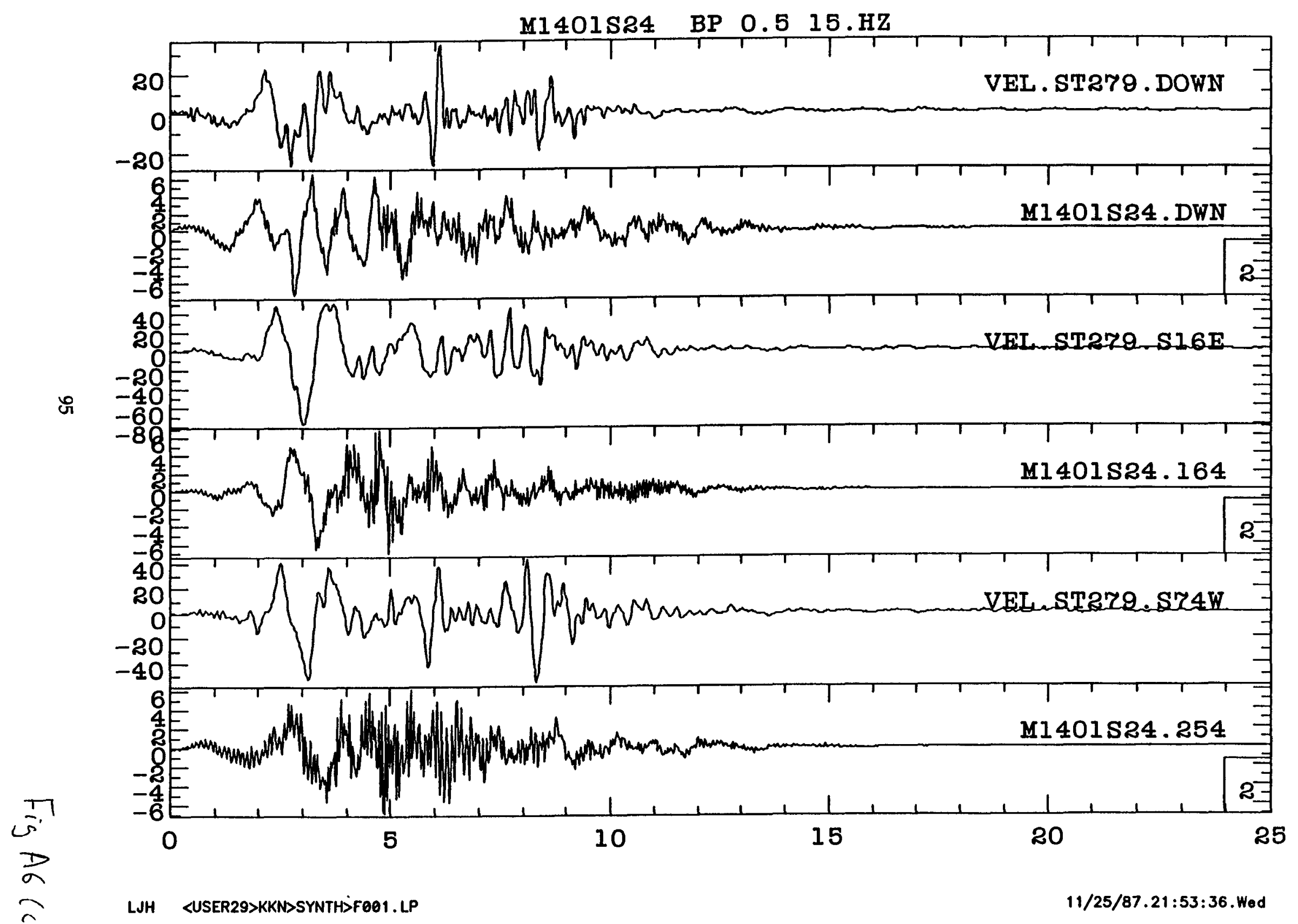


STNTHESIS OF EVENT 1401 AT STATION 24

HYPOCENTER LOCATION

$34.4040 \quad 118.4098 \quad 10.00$

SYNTHESIZED FAULT PARAMETERS

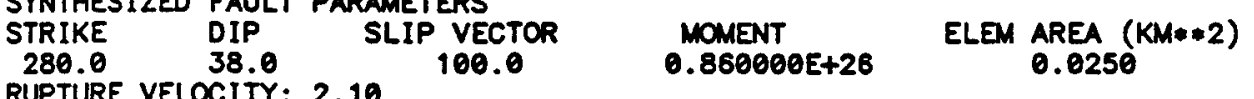

RUPTURE VELOCITY: 2.10

P VELOCITY: VP $=3.0+H * 0.20$ FOR TT CORR OF SOURCE EVENTS

ORIENTATION OF SYNTHESIZED TRACES

POSITIVE ON OUTPUT: DWN, 164.0,254.0 DEGREES FROM NORTH

RADIATION CORRECTION FACTOR APPLIED FOR DIFFERENCES BETWEEN

SOURCE EVENTS AND. ELEMENTS. BUT LIMITED TO -.4 TO +.4

STRESS DROP 46.5

\begin{tabular}{|c|c|c|c|c|c|c|c|c|}
\hline $\begin{array}{c}\text { OURCE } \\
57 \\
59 \\
60 \\
60 \\
60 \\
60 \\
60 \\
60 \\
60 \\
60 \\
61 \\
60\end{array}$ & $\begin{array}{l}\text { E EVENTS } \\
2336 \\
2251 \\
816 \\
1023 \\
1115 \\
1153 \\
1424 \\
1451 \\
2008 \\
2201 \\
302 \\
234\end{array}$ & $\begin{array}{l}34.3553 \\
34.3078 \\
34.4072 \\
34.3313 \\
34.2995 \\
34.4202 \\
34.4123 \\
34.3565 \\
34.3917 \\
34.3768 \\
34.3950 \\
34.4523\end{array}$ & $\begin{array}{l}\text { LOCATION } \\
118.3360 \\
118.3675 \\
118.3622 \\
118.3665 \\
118.4723 \\
118.4677 \\
118.3923 \\
118.3003 \\
118.3673 \\
118.4413 \\
118.4160 \\
118.4508\end{array}$ & $\begin{array}{r}7.24 \\
10.09 \\
13.03 \\
1.49 \\
6.65 \\
9.51 \\
10.46 \\
8.54 \\
9.96 \\
7.61 \\
7.89 \\
11.14\end{array}$ & $\begin{array}{c}\text { MOMENT } \\
0.692000 E+21 \\
0.126150 E+22 \\
0.154870 E+22 \\
0.530000 E+20 \\
0.554000 E+20 \\
0.476470 E+22 \\
0.153650 E+22 \\
0.849300 E+21 \\
0.273750 E+22 \\
0.922200 E+21 \\
0.377390 E+22 \\
0.200150 E+22\end{array}$ & $\begin{array}{l}\text { STRIKE } \\
262 . \\
326 . \\
253 . \\
213 . \\
296 . \\
235 . \\
268 . \\
279 . \\
262 . \\
275 . \\
300 . \\
238 .\end{array}$ & $\begin{array}{l}\text { DIP } \\
30 . \\
66 . \\
38 . \\
62 . \\
29 . \\
28 . \\
52 . \\
24 . \\
30 . \\
50 . \\
70 . \\
32 .\end{array}$ & $\begin{array}{r}\text { SV } \\
90 . \\
147 . \\
90 . \\
49 . \\
118 . \\
80 . \\
141 . \\
90 . \\
42 . \\
-24 . \\
142 . \\
90 .\end{array}$ \\
\hline
\end{tabular}

STATION: 24 SLAT= 34.3333 SLON 118.3986

RUPTURE AREA IS ELLIPITICAL: MAJOR AXIS $25.0 \mathrm{KM}$, MINOR AXIS $10.0 \mathrm{KM}$, ECCENTRICITY IS 0.60 ANGLE OF PERHELION OF MAJOR AXIS CLOCKWISE FROM STRIKE OF FAULT IS 285.0. FOUR POINTS ON ELLIPSE AT 90 DEG INTEVERALS FROM 0 DEG UP DIP: $19.06 .95 .19 .5 \mathrm{kM}$.

VH $=4.1$, RUPTURE HEALING AT P VELOCITY

\begin{tabular}{|c|c|c|c|c|}
\hline $\begin{array}{l}\text { RING: } \\
\text { RING: } \\
\text { RING: } \\
\text { RING: } \\
\text { RING: } \\
\text { RING: } \\
\text { RING: } \\
\text { RING: } \\
\text { RING: } \\
\text { RING: } \\
\text { RING: } \\
\text { RING: } \\
\text { RING: } \\
\text { RING: } \\
\text { RING: }\end{array}$ & $\begin{array}{r}2 \\
3 \\
4 \\
5 \\
6 \\
7 \\
8 \\
9 \\
10 \\
11 \\
12 \\
13 \\
14 \\
15 \\
16\end{array}$ & $\begin{array}{l}7 \\
14 \\
20 \\
27 \\
33 \\
39 \\
46 \\
52 \\
58 \\
65 \\
71 \\
77 \\
84 \\
90 \\
96\end{array}$ & $\begin{array}{l}\text { ELEMENTS } \\
\text { ELEMENTS } \\
\text { ELEMENTS } \\
\text { ELEMENTS } \\
\text { ELEMENTS } \\
\text { ELEMENTS } \\
\text { ELEMENTS } \\
\text { ELEMENTS } \\
\text { ELEMENTS } \\
\text { ELEMENTS } \\
\text { ELEMENTS } \\
\text { ELEMENTS } \\
\text { ELEMENTS } \\
\text { ELEMENTS } \\
\text { ELEMENTS }\end{array}$ & $\begin{array}{l}\text { RADIUS- } \\
\text { RADIUS- } \\
\text { RADIUS- } \\
\text { RADIUS- } \\
\text { RADIUS- } \\
\text { RADIUS- } \\
\text { RADIUS- } \\
\text { RADIUS- } \\
\text { RADIUS- } \\
\text { RADIUS- } \\
\text { RADIUS- } \\
\text { RADIUS- } \\
\text { RADIUS- } \\
\text { RADIUS- } \\
\text { RADIUS- }\end{array}$ \\
\hline
\end{tabular}


RING: 17

RING: 18 ,

RING: 19 ,

RING: 20 ,

RING: 21 ,

RING: 22 ,

RING: 23 ,

RING: 24 .

RING: 25

RING: 26 ,

RING: 27 ,

RING: 28,

RING: 29 ,

RING: 30 ,

RING: 31 ,

RING: 32 ,

RING: 33 ,

RING: 34 ,

RING: 35 ,

RING: 36 ,

RING: 37 ,

RING: 38 ,

RING: 39 ,

RING: 40 .

RING: 41 ,

RING: 42 ,

RING: 43

RING: 44,

RING: 45 ,

RING: 46

RING: 47 ,

RING: 48 ,

RING: 49 ,

RING: 50

RING: 51 .

RING: 52 ,

RING: 53 ,

RING: 54.

RING: 55 .

RING: 56,

RING: 57,

RING: 58,

RING: 59 ,

RING: 60 ,

RING: 61,

RING: 62 ,

RING: 63,

RING: 64 .

RING: 65 .

RING: 66 .

RING: 67.

RING: 68,

RING: 69 ,

RING: 70 ,

RING: 71 ,

RING: 72

RING: 73 .

RING: 74 ,

RING: 75 ,

RING: 76,

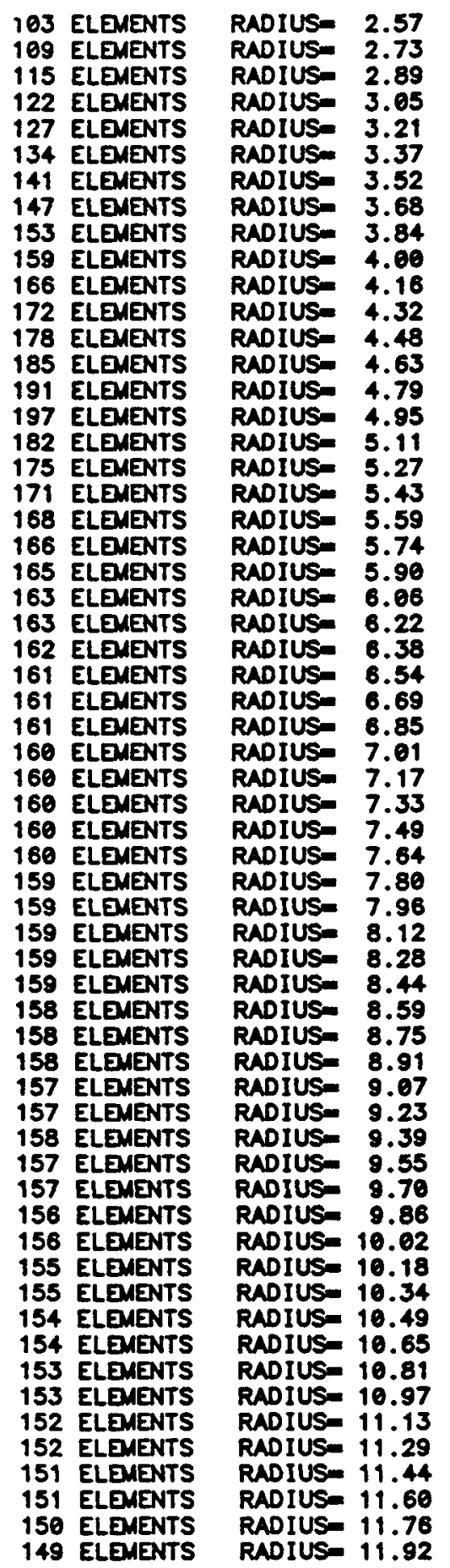

103 ELEMENTS

ELEMENTS

127 ELEAENTS

34 ELENENTS

147 ELEVENTS

33 ELEMENTS

ELIMENTS

172 ELEMENTS

178 ELENENTS

197 ELEMENTS

171 ELENENTS

168 ELEMENTS

65 ELEMENTS

163 ELEMENTS

ELENENTS

161 ELEMENTS

61 ELEMENTS

160 ELENENTS

ELEVENTS

160 ELEMENTS

ELONENTS

159 ELEMENTS

159 ELEMENTS

158 ELEMENTS

ELEMENTS

157 ELEMENTS

ELEMENTS

155 ELEMENTS

ELEMENTS

153 ELEMENTS

153 ELEMENTS

152 ELEMENTS

149 ELEMENTS

RADIUSO 11.92 


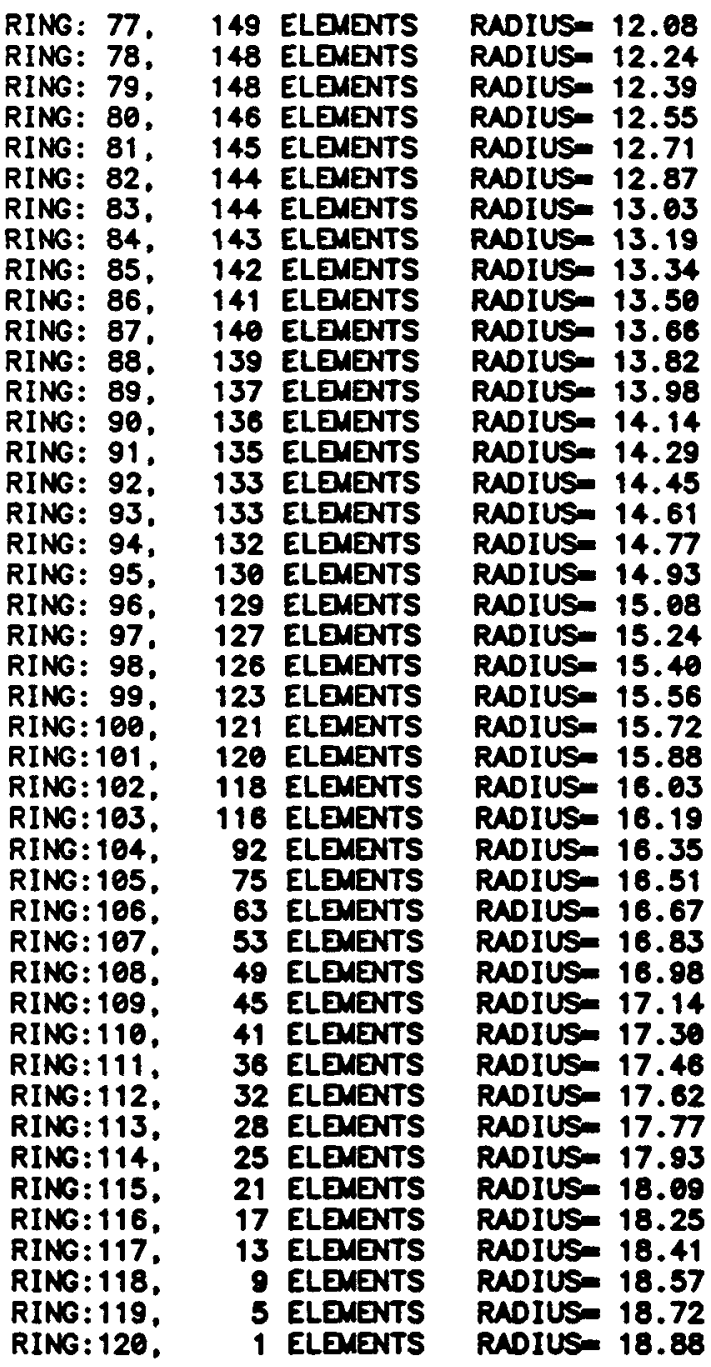

TOTAL MMBER OF ELEMENTAL AREAS IS 14423 TOTAL FAULTING DURATION: 11.50

**** ELEMENTS ****

HYPOCENTER: $\quad$ RISTM $=3.61 \quad$ DISPm $128.74 \mathrm{CM}$

SYNTHESIS IS BY CONVLUTION OF SOURCE EVENTS WITH SLIP FUNCTION,

DECONVOLVED WITH STEP FUNCTION. EACH SOURCE EVENT IS SPLIT BETWEEN P AND $S$, AND CONPUTATION IS DONE IN FREQUENCY DOMAIN. FFT FOR EACH SOURCE EVENT ONCE. RANP DISPLACEMENT FUNCTION - MODIFIED KOSTROV

SLIP ANPLITUDE AND RISE TIME CONSTRAINED BY SELF-SIMILAR KOSTROV SLIP ELENENT DISPLACEMENT (LEFT TO RIGHT) 
FIRST 160 ELEMENTS

\begin{tabular}{|c|c|c|c|c|c|c|c|}
\hline $\begin{array}{l}28.741 \\
30.046 \\
28.327 \\
29.789 \\
26.412\end{array}$ & $\begin{array}{l}129.044 \\
129.431 \\
129.269 \\
128.889 \\
127.325 \\
131.139 \\
126.545 \\
126.623 \\
132.260 \\
130.929 \\
123.467 \\
127.631 \\
133.243 \\
133.103 \\
124.762 \\
122.529 \\
131.322 \\
133.535 \\
133.989\end{array}$ & $\begin{array}{l}128.204 \\
128.573 \\
130.059 \\
127.993 \\
128.370 \\
131.756 \\
125.603 \\
127.667 \\
132.070 \\
130.007 \\
123.153 \\
128.874 \\
133.000 \\
132.486 \\
123.838 \\
123.231 \\
132.481 \\
133.197 \\
133.136 \\
124.226\end{array}$ & $\begin{array}{l}13 \\
12 \\
125 \\
13 \\
12 \\
128 \\
13 \\
128 \\
12 \\
13 \\
132 \\
13 \\
12 \\
12 \\
13 \\
13 \\
132\end{array}$ & $\begin{array}{l}13 \\
12 \\
13 \\
13 \\
12 \\
12 \\
13 \\
12 \\
12 \\
13 \\
13 \\
13 \\
12 \\
12 \\
13 \\
13\end{array}$ & $\begin{array}{l}13 \\
12 \\
13 \\
13 \\
12 \\
13 \\
13 \\
12 \\
12 \\
13 \\
13 \\
12 \\
12 \\
12 \\
13 \\
13 \\
13\end{array}$ & & \\
\hline
\end{tabular}
YOU GET WRAP-AROUND IN MAIN SEISIOERAM

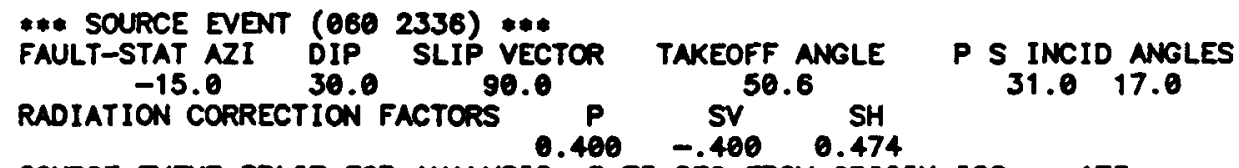

SOURCE EVENT SPLIT FOR ANALYSIS 3.75 SEC FROM ORIGIN ISS = 175

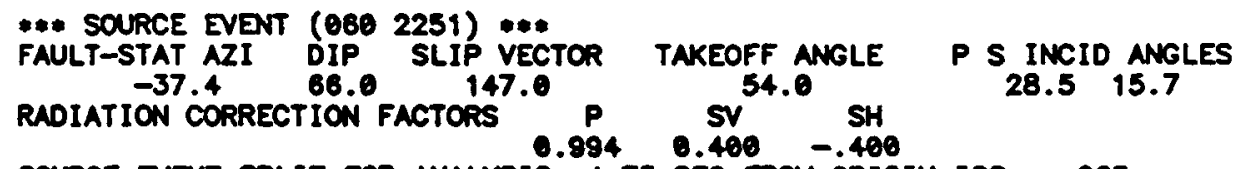

*** SOURCE EVENT (060 0816) ***

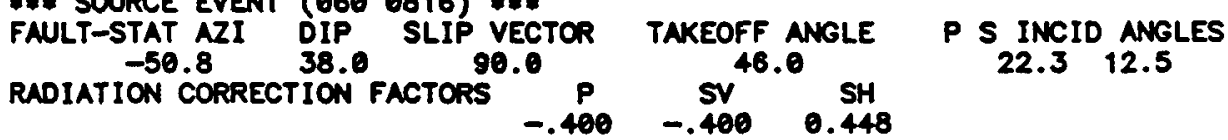

SOURCE EVENT SPLIT FOR ANALYSIS 5.50 SEC FRON ORIGIN ISS - 346

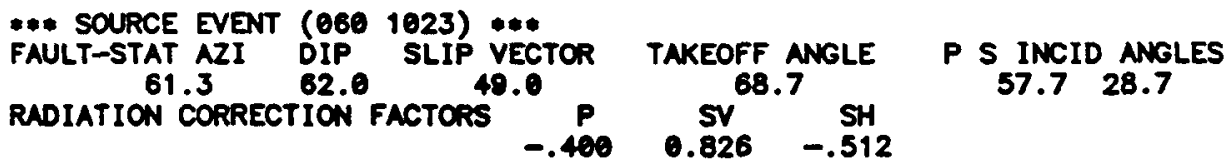


SOURCE' EVENI SPLIT FOR ANALYSIS 4.20 SEC FROM ORIGIN ISS = 202 $-.400$

$$
0.400 \quad-.400
$$

.*. SOURCE EVENT (060 0234) \#...

FAULT-STAT AZI DIP SLIP VECTC RADIATION CORRECTION FACTORS

SOURCE EVENT SPLIT FOR AMLYSIS 8.00 SEC FROM ORIGIN ISS - 300

P S INCID ANGLES

$32.3 \quad 17.7$

MONENT OF ACTUAL EVENT $0.860000 E+26$

MOMENT OF MOOELED EVENT $0.119899 E+27$

SLAT $=34.3333$ SLON 118.3986 DIS- 5.10 AZINV 205.1

$0.29-0.29-1.01 \quad 0.72203$ LOOP

$\begin{array}{lllllll}0.26 & 0.33 & 1.26 & -1.83 & 202 & L & 100 P\end{array}$

IA 64 AB 2.867 ADIS= 2.592 


\section{APPENDIX B}

Following is a partial listing of the code for EMPSYN and is not sufficient to synthesize seismograms. 


\section{Appendix $B$ \\ empsyn.f}

C

PROGRAM EMPSYN

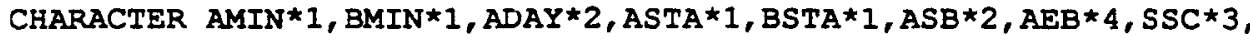
$\star$ AHOUR $\star 1$, BHOUR $\star 1$, FNSE $1 * 17$, FNSE $2 * 17$, FNSE $3 * 17$, BLANK $\star 1$, ZERO $* 1$, ANS $2 \star 3$, $\star \mathrm{ANS} 1 * 1, \mathrm{ALPN} * 80, \mathrm{ANS} * 3, \mathrm{NNSF} * 16, \mathrm{NNSE} 1 * 30, \mathrm{NNSE} 2 \star 8, \mathrm{NNSF} 3 * 30, \mathrm{DWN} * 3, \mathrm{RDC} * 3$ $\star$, SLP *3, ANS $3 * 3$, ANS $4 * 3$, ANS $5 * 3$, ANS $6 * 3, S I M * 3, \operatorname{ALPHSYN} * 4, \operatorname{COMP} * 3$ DIMENSION BIAT $(60), \operatorname{BION}(60), \mathrm{BH}(60), \mathrm{BM}(60), \mathrm{EA}(60), \mathrm{EB}(60), \mathrm{SPT}(60)$, $\star \operatorname{BSTR}(60), \operatorname{BDP}(60), \operatorname{BSV}(60), \mathrm{FL}(301), \operatorname{RDIS}(30000), \operatorname{RDPP}(30000)$, $\star \operatorname{RLON}(30000), \operatorname{RH}(30000), \operatorname{RAZM}(30000), \operatorname{TRAZ}(9000), \operatorname{TRAR}(9000), \operatorname{TRAT}(9000)$

*, RISTM (30000), INEL (30000), ELTIM(30000), DTIMP (30000), RLAT (30000), *ETRZ (9000), ETRR(9000), ETRT (9000), TRZ (9000), TRR (9000), TRT (9000), $\star T Z P Z(9000), \operatorname{TZPR}(9000), \operatorname{TZPT}(9000), \operatorname{TZSZ}(9000), \operatorname{TZSR}(9000), \operatorname{TZST}(9000)$

COMMON TRAZ, TRAR, TRAT, ETRZ, ETRR, ETRT, TRZ, TRR, TRT, TZPZ, TZPR, *TZPT, TZSZ, TZSR, TZST, RION, RLAT, RH, RAZM, RISTM, INEL, ELTIM, DTIMP , *RDIS, RDPP INTEGER SB,EA, EB, EAA, EBB INTEGER 2 SYM1, SYM2, SYM3, SYM6, SYM11, SYM15, SYM16 REAL LN, ML, INT, MO COMPIEX TZPZ, TZPR, TZPT, TZSZ, TZSR, TZST, ESLP, ZPHP, ZPHS, ETRZ, ETRR, ^ETRT, TRZ, TRR, TRT, ETRA1, ETRA2, ETRA3, TR1, TR2, TR3, FFAC1, FFAC2, FFAC3, *ELAC1, ELAC2, ELAC3, RPFAC, RRFAC, RTFAC

DATA ANS1, ANS2, BLANK, ZERO, 'Y', 'YES',' ', 'O'/

C

DATA ANS3, ANS4, ANS5, ANS6/'KSV', 'HSK', 'SPC', 'TTM'

c

$P I 2=6.28318531$

C SEISMIC QUALITY QUOTIENT

$Q=250.0$

$\mathrm{GC}=(1 .-1 \cdot 1297.) \star \star 2$

$\mathrm{DR}=\mathrm{PI} 2 / 360$.

C

$\operatorname{MAX}=10000$

WMINY $=0.0$

WMAXY $=10.75$

WMAXX $=14.4275$

WMINX $=0.0$

C

CALI PLOTS (WMAXX, WMAXY, 1)

C FILTER FOR CONVOLVED TRACE

CPLT $=0.0$

rtmhsk $=1.0$

EILOW $=0.0$

FILHI $=100.0$

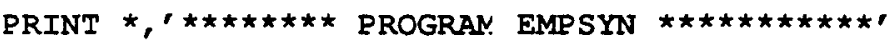

WRITE $(6,302)$

302 FORMAT $(/ /)$

304 FORMAT (A3)

PRINT *,'SYNTHESIZES EARTHQUAKES WITH EMPIRICAL GREEN FUNCTIONS'

PRINT *,",

PRINT *,'PARAMETERS NEEDEI, FOR SYNTHESIS:'

PRINT $*, \prime \prime$

PRINT *,'FL: ARRAY OF DISTANCES FROM HYPO TO EDGE OF RUPTURE'

PRINT *,' IN KILOMETERS CLOCKWISE FROM $O$ DEG UPDIP'

PRINT *,',

PRINT *', IP: NUMBER OF VALUES FOR EL'

PRINT *,'IP = 1 FOR CIRCULAR RUPTURE, UNLESS ENCOUNTERS SURFACE' WRITE $(6,997)$

997 FORMAT (/" IP = 3 FOR ELLIPTICAL RURTURE AREA - INPUT IN ORDER:'/ * LENGTH OF MAJOR AXIS, ECCENTRICITY, AND ANGLE CLOCKWISE'

*/, FROM DIRECTION OF' STRIKE FOR PERIHELION OF MAJOR AXIS $/ 1$

PRINT $\star, '$ IP $=4$ FOR RECTAJIGULAR RUPTURE AREA'

PRINT *,' '

PRINT *,' INPUT VALUES FOR FI ARE IN FILE: FAULT'

PRINT *,' FORMAT FOR FIIE' IS FREE FORMAT: IP FIRST LINE' 
112 FORMAT (/,'STRESS DROP ',E5.1)

113 FORMAT (1X, F5.1, 5X, F5.1,8X, F6.1, 7X, E13.6, 9X, F6.4)

121 FORMAT (1X, 6E13.6)

123 FORMAT (1X)

124 FORMAT (/,'TOTAL NUMBER OF ELEMENTAL AREAS IS ', I5, $\star 4 X$, 'MAXIMUM RADIUS ', E5.2,' $\mathrm{KM}^{\prime}$ )

129 FORMAT (8F9.3)

130 FORMAT('SAMPLE RADIATION CORRECTION FACS, 10 ELEMS AT HYPOCENTER' */, ELPP ELSV ELSH')

131 FORMAT (3X, F5.3, 6X, F5.3, 6X, F5.3)

132 FORMAT/'SLIP FUNCTION INCREMENTED BY DIGITIZING RATE'/,

*' SOURCE EVENTS AND SYNTHESIZED OUTPUT IIMITED TO ', I 4,' POINTS')

125 FORMAT (i1, i1, il, i1)

126 FORMAT ('MO',E13.6/,'AMO ', IX,E13.6)

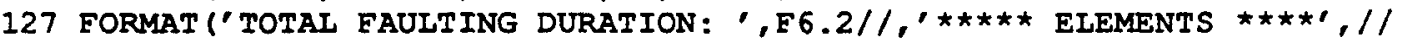
*,'HYPOCENTER:', 15X,'RISTM = ',F6.2,' DISP= ',F7.2,' CM'/,

*'STATION SOURCE PULSE DURATION: ',F5.2)

128 EORMAT ('RING:',I3,',',2X,I4,' ELEMENTS RADIUS= ',F5.2)

133 FORMAT (//, VH $=$ ',F3.1,', RUPTURE HEALING AT S VELOCITY')

134 FORMAT $\left(/ /,{ }^{\prime}\right.$ VH $=$,F3.1,', RUPTURE HEALING AT P VELOCITY')

135 FORMAT ('ELEMENT DISPLACEMENT (LEFT TO RIGHT)'/,

*' EIRST ',I3,' ELEMENTS')

418 FORMAT ('RAMP DISPLACEMENT FUNCTION - MODIEIED KOSTROV'//,'SLIP AMP *IITUDE AND RISE TIME CONSTRAINED BY SELE-SIMILAR KOSTROV SIIP')

419 FORMAT ///'TWO DIMENSIONAL HASKEI RUPTURE MODEL WITH RAMP SIIP FUNC *TION'//'EACH ELEMENT HAS SAME DISPLACEMENT:',F5.1,' CM'/'RISE TIME * =',F5.2,' TOTAL FAULTING DURATION ',E5.2\%,

*'STATION SOURCE PULSE DURATION: ',F5.2)

C

C$$
\text { C }
$$

EA (I), EB (I) DAYS HRS MINS OF SOURCE EVENTS EAA, EBB TEMPORARY USAGE OF EA(I) AND EB(I) SPT(I) TIME TO SPLIT SEISMOGRAM BETWEEN $P$ AND $S$ WAVES ALAT, ALON, AH: HYROCENTER LOCATION SLAT, SLON: STATION LOCATION BIAT (I), BLON(I) , BH(I) : ARRAY OF SOURCE EVENTS BLAT1, BLON1, BH1: TEMPORARY VALUES FOR SOURCE EVENTS BAZM, BDIS: NOT INDEXED, RESET FOR EACH SOURCE EVENT RIAT (KK) , RION (KK) , RH (KK) : ELEMENTAL LOCATIONS RDIS (KK), RAZM (KK) : ELEMENT-STATION EPICENTRAL VALUES RDPP (KK) : FAULT DISPLACEMENT AT ELEMENT

INEL (KK) : INDICE VALUE OF SOURCE EVENT FOR ELEMENT KK DTIMP (KK) : IS DELAY TIME OF ILEMENTAL SOURCE P ARRIVALS DUE TO DIFEERENCE IN LOCATION OF SOURCE EVENT AND ELEMENT ELTIM(KK): IS ELEMENT DELAY TIME DUE TO RUPTURE TIME TO ELEMENT RISTM(KK) : IS RISE TIME OF ELEMENT, COMPUTED FOR KOSTROV SLIP FUNC SFO: TIME DELAY FROM ORIGIN FOR BEGINING OUT SYNTHESIZED TRACE STD: STRESS DROR, computed to fit requested moment

BSTR, BDP, BSV, BM: FAULT PARAMETERS FOR SOURCE EVENT, FROM CATLOG STK, DP, SV: FAULT PARAMETER FOR EVENT TO BE MODELED

TRAZ, TRAR, TRAT: SOURCE EVENT, TRACES READ IN ONE AT A TIME AND USED FOR ALL ELEMENTS USING THIS SOURCE EVENT.

ETRZ,ETRR,ETRT: ELEMENTAL SOURCE TRACE AFTER TIME FUNCTION STACKING

TZPZ, TZPR, TZPT: $P$ PORTION OF SEISMOGRAM USED IN CONVOLUTION

TZSZ,TZSR, TZST: S PORTION OF SEISMOGRAM AFTER CONVOLUTION THESE ARE PHASE SHIFTED BEFOR ADDING TO FINAL 
C SOURCE EVENTS AND ELEMENTAL SOURCES ARE CORRECTED FOR DIFFERENCES

C IN GEOMETRICAL SPREADING AND ATTENUATION: THIS ALLOWS A SINGLE

C EVENT TO BE USED AS A SITE RESPONSE FUNCTION; AND CORRECTS FOR LARGE

C SPATIAL DIFERENCES OF SOURCE EVENTS AND ELEMENTAL SOURCES

C IF RDC = NO THEN THERE IS NO RADIATION CORRECTION FACTOR APPLIED

C FOR DIFFERENCES BETWEEN SOURCE EVENTS AND ELEMENTS

C IF RDC = YES THEN RADIATION CORRECTION FACTOR APPLIED BUT LIMITED TO

C -.4 TO +.4

$\mathrm{C}$

C CHARACTERS SPECIFIED FOR NNSF MUST BE EXACT

NNSF 1 = 'fault'

OPEN (UNIT $=10$, FILE=NNSF 1$)$

NNSF1 = 'synout'

OPEN (UNIT $=11$, FILE $=$ NNSF 1$)$

NNSF1 = ' sources'

OPEN (UNIT $=7$, FILE $=$ NNSE 1 )

PRINT *,'WHAT IS THE HOURS-MINS (A4) OF MAIN EVENT'

$\operatorname{READ}(5,125) 11,12,13,14$

ALPHSYN $=\operatorname{char}(11+48) / / \operatorname{char}(12+48) / / \operatorname{char}(13+48) / / \operatorname{char}(14+48)$

C NNSE $3=$ ' $m^{\prime} / /$ ALPHSYN//' $s$ ' //ASB

C OPEN (UNIT $=9$, FILE=NNSE3)

$\operatorname{READ}(10, *)$ IP

$\operatorname{READ}(10, *) \quad(F L(I), I=1, I P)$

CLOSE (10)

WRITE $(11,102)$ ALPHSYN, SB

WRITE (11, 101) ALAT, ALON, AH

WRITE $(11,104)$

WRITE $(11,113)$ STK, DP, SV, MO, ELA

WRITE (11,111) VR, VPP, COMP, SAZM, SAZMM

IF (RDC.EQ.ANS1 . OR.RDC.EQ.ANS2) $\operatorname{WRITE}(11,196)$

IF (RDC.NE.ANS1.AND.RDC.NE.ANS2) WRITE $(11,197)$

196 FORMAT (/,'RADIATION CORRECTION FACTOR APPIIED FOR DIFFERENCES BETW ${ }^{*}$ EEN' $/$ 'SOURCE EVENTS AND ELEMENTS, BUT LIMITED TO -.4 TO $+.4 \%$ '

197 FORMAT (/,'NO RADIATION CORRECTION FACTOR APPIIED FOR DIFFERENCES B *ETWEEN' $/$, SOURCE EVENTS AND ELEMENTS. THIS THEN USES EMPIRICAL GR *EEN'/" FUNCTIONS AS SITE RESPONSE FUNCTIONS'/1

if (SLP.EQ.ANS6) write $(11,198)$

if (SLP.EQ.ANS5) write (11, i99)

198 fOrmat ('NO CORRECTION MADE FOR GEOMETRICAL SPREADING OR ATTENUATIO $\left.{ }^{*} \mathrm{~N}^{\prime} / /\right)$

199 FORMAT('CORRECTIONS APPLIED FOR GEOMETRICAI SPREADING AND ATTENUAT *ION'/)

IF (IP.GT.4) WRITE(11,109) IP

IF (IP.GT.4) WRITE (11, 108) (FL(I), I = 1, IP)

IF (IP.EQ.1) WRITE (11,995) FL(1)

IF (IP .EQ.4) WRITE (11,994) FL(1),FL (2), FL (3), FL (4)

994 FORMAT ('RECTANGULAR FAULT RUPTURE. DISTANCES TO EDGE CLOCKWISE FR *OM HYPOCENTER: 'F4.1,1X,F4.1,1X,F4.1, 1X, F4.1)

995 FORMAT ('CIRCULAR FAULT RUPTURE. RADIUS: ',F4.1)

WRITE $(11,107)$

$\mathrm{DP} 1=(\mathrm{DP} / 360). * \mathrm{PI} 2$

$\mathrm{STK1}=(\mathrm{STK} / 360). * \mathrm{PI} 2$

$\mathrm{NE}=0$

C

C SET PARAMETERS FOR SOURCE EVENTS

DO $10 I=1,60$

$\operatorname{READ}(7, \star, E N D=11) \quad E A A, E B B, S P P, B L A T 1, B L O N 1, B H 1, B M 1, B S T R 1, B D P 1, B S V 1$

IF(SPP.LT.1..OR.SPR.GT.20.) PRINT *,'BAD S-P SPIIT TIME'

IF (SPP.LT.1..OR.SPP.GT.20.) GO TO 2000

$\mathrm{BM}(\mathrm{I})=\mathrm{BM} 1 * 1.0 \mathrm{E}+20$

WRITE $(11,106)$ EAA, EBB, BLAT1, BLON1, BH1, BM(I),BSTRI, BDP 1, BSV1

$\operatorname{BLAT}(I)=$ BLAT1

$\operatorname{BLON}(I)=$ BLON1 


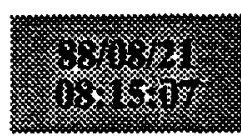

hutchs

empsyn.f

$\mathrm{BH}(\mathrm{I})=\mathrm{BH} 1$

$E A(I)=E A A$

$\mathrm{EB}(\mathrm{I})=\mathrm{EBB}$

$\operatorname{SPT}(I)=$ SPP

$\operatorname{BSTR}(I)=\operatorname{BSTRI}$

$\operatorname{BDP}(I)=B D P I$

$\operatorname{BSV}(I)=\mathrm{BSVI}$

$\mathrm{NE}=\mathrm{NE}+1$

10 CONTINUE

11 CLOSE (7)

WRITE $(11,110)$ SB, SLAT, SLON

C

C INDEX ELEMENTAL AREAS, IDENTIFY SOURCE EVENTS, SET DELAY TIMES

C

IF (IP.EQ.1) GO TO 116

IF (IP.EQ.3) GO TO 114

IF (IP.EQ.4) GO TO 117

$I P A=0$

GO TO 118

$116 \mathrm{IP}=300$

DO $115 \mathrm{I}=2, I P$

$115 \mathrm{FI}(\mathrm{I})=\mathrm{FL}(1)$

$I P A=1$

GO TO 118

114 IP $=300$

$\mathrm{AAA}=\mathrm{FL}(1)$

$A A=F I(1) / 2$.

$E C=F I(2)$

ANN1 $=F I(3)$

ANN2 $=F I(3) * .01745329$

$\mathrm{BB}=\mathrm{AA} \star \operatorname{SQRT}(1.0-\mathrm{EC} \star \star 2)$

$\mathrm{BBB}=2.0 * \mathrm{BB}$

DO $122 I=1, I P$

$\operatorname{ANN}=\operatorname{PI2} / 4 .-\operatorname{ANN} 2-\operatorname{FLOAT}(I-1) \star \mathrm{PI} 2 / 300.0$

$122 F I(I)=A A *(1 .-E C * \star 2) /(1 .+E C * \operatorname{COS}(A N N))$

$I P A=3$

WRITE $(6,999)$ AAA, BBB, EC, ANN1, FL (1), EL (76), EL (151), FL (226)

WRITE (11, 999) AAA, BBB, EC, ANN1, EI (1), FI (76), FI (151), FL (226)

999 FORMAT ('RUPTURE AREA IS ELIIPITICAL: MAJOR AXIS ',F4.1,' KM, MINOR

* AXIS ',F4.1,' KM,'/,'ECCENTRICITY IS ', F4.2,' ANGLE OF PERHELION

* OF MAJOR AXIS CLOCKWISE FROM STRIKE OE' $\%$

*'FAULT IS ',F5.1,'. FOUR POINTS ON ELLIPSE AT 90 DEG INTEVERALS F

*ROM 0 DEG UP DIP: ' /,F4.1,1X,F4.1,1X,F4.1,1X,F4.1,' KM.'/)

C WRITE $(11,998)(F L(I), I=1, I P)$

998 FORMAT (16F5.1)

GO TO 118

117 IP $=300$

$F L 1=F L(1)$

$F L 2=F L(2)$

$F I 3=F L(3)$

FI4 $=F I(4)$

ANN1 $=\operatorname{ATAN2}(F L 2, F I 1)$

ANN2 $=P I 2 / 4 .+$ ATAN2 (EL3, FL2)

ANN3 $=P I 2 / 2 .+$ ATAN2 (FI4,FI3)

ANN4 $=.75 *$ PI2 + ATAN2 (FLI, FL4)

DO $119 \mathrm{I}=1$, IP

ANN $=$ FIOAT $(I-1) \star \mathrm{PI} 2 / 300.0$

IF (ANN . GE . ANN 4 . OR. ANN. LE . ANN1) FL (I) $=$ ABS (FL1/COS (ANN))

IF (ANN.GT . ANN1 . AND . ANN. LE . ANN2) FI (I) $=$ ABS (FI2/SIN (ANN))

IF (ANN. GT . ANN2 . AND . ANN . LE . ANN3) FI (I) $=A B S(F L 3 / C O S(A N N)$ )

IF (ANN . GT . ANN3 . AND . ANN . LT . ANN4) FL (I) $=A B S(F L 4 / S I N(A N N)$ )

119 CONTINUE

$I P A=4$

118 ANG = PI2/FLOAT (IP) 
$R 1=0.0$

$R 2=\operatorname{SQRT}(E L A \star 2 . / P I 2)$

$\mathrm{R} 3=0.0$

ANG4 $=$ PI2 $/ 4.0$

C

C GO TO 160 FOR EACH RING

C KI INDEX DO LOOP 140 TO GO FROM NEXT ELEMENT IN RING

C KKI SETS VALUE OF KI FOR DO LOOP

C JI INDEX FOR FL(JI) AND ANGI, ANG2

C KJ LIMITS CHECK ON ELEMENTS, ENDS GO TO 160 LOOP

C KK NUMBER OF ELEMENTAL AREAS

C

$$
\mathrm{KK}=1
$$

$\mathrm{DH} 1=100.0$

C

C FACTORS TO GET LATITUDE LONGITUDE OF ELEMENTS FROM R3: TRIG $=S Q R T(1.0-(6.76866 E-3 * \operatorname{SIN}($ ALAT *PI2/360.) $\star \star 2))$ RHOM $=6.3782 \mathrm{E}+3 *(1.0-6.76866 \mathrm{E}-3) / \mathrm{TRIG} * * 3$

C RHON $=6.3782 \mathrm{E}+3 / \mathrm{TRIG}$

C SET VALUES EOR EIRST ELEMENTAL AREA, GET AZIMUTHAL DISTANCE C

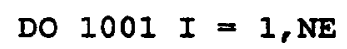




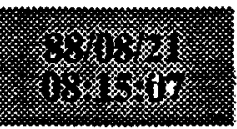

\section{hutchs}

\section{empsyn.f}

IF (VH.EQ.VH2 . AND.SIM.EQ.ANS3) WRITE (11,134) VH

IF (IPA.NE.1.and.ipa.ne.3) CALI RISTIM(VR, VH, EL, IP, R3, ANG4, RSTM)

if (ipa.eq.3) rstm $=(1 . / v r+1 . / v h) *(a a *(1 .-e c * \star 2)-r 3 * e c *$

$\star \cos (p i 2 / 4$. -ang 4-ann2) $r 3$ )

IF (IPA.NE.1) RISTM(1) = RSTM

$\operatorname{IF}(I P A . E Q .1) \operatorname{RISTM}(1)=F L(1) *(1 . / \mathrm{VR}+1 . / \mathrm{VH})$

$\operatorname{RDPP}(1)=0.81 * S T D * \operatorname{SQRT}(\operatorname{RISTM}(1) * \star 2)$

amo $=\operatorname{rdpp}(1) * I g d * e l a$

IF (RDPP (1).GT.CPLT) ICPLT $=1$

IF (RDPP (1) . GT.CPLT) CPLT = RDPP (1)

12 if (s lm.ne.ans3) ristm(1) = rtmhsk

DURTIM $=$ RISTM(1)

sdur $=r 2 / v r+$ ristm(I)

$\mathrm{RH}(1)=\mathrm{AH}$

$\operatorname{RIAT}(1)=$ ALAT

RION $(1)=A L O N$

$\operatorname{KKII}=0$

$\mathrm{KKIII}=1$

$160 \mathrm{KKI}=1$

$\mathrm{KKKII}=\mathrm{KK}-\mathrm{KKII}$

IF (KKII.NE .0) WRITE $(11,128)$ KKIII, KKKII, R3

IF (KKII.NE.0) WRITE $(6,128)$ KKIII, KKKII, R3

$K I=1$

$\mathrm{KJ}=0$

$\mathbf{R} 1=\mathbf{R} 1+\mathbf{R} 2$

C

C RI IS RADIUS OF PREVIOUS EXTENT OF SOURCE AREA

C R2 IS THICKNESS OF CURRENT RING BEING EXAMINED

C R3 IS FIRST ARC LENGTH OF ELEMENT THEN BECOMES RADIUS TO CENTER OF ELEMENT

C $r 33$ is radial distance to edge of element

C JN IS NUMBER OE ELEMENTS PER RING

C INT IS DISTANCE TO EDGE OF FAULT IN RADIAL DIRECTION OF ELEMENT

C

DO $120 \mathrm{JN}=1,1000$

$\mathrm{R} 2=-\mathrm{R} 1+\operatorname{SQRT}(\mathrm{R} 1 * \star 2+(\mathrm{ELA} * 2 . * \mathrm{FLOAT}(\mathrm{NN}) / \mathrm{PI} 2))$

$R 3=P I 2 *(R 1+R 2 / 2.0) / F L O A T(J N)$

IF (R2.GT.R3) GO TO 30

120 CONTINUE

30 ANG3 $=$ PI2/FLOAT (JN)

$\mathrm{R} 3=\mathrm{R} 1+\mathrm{R} 2 / 2.0$

$r 33=r 1+r 2$

$\operatorname{KKIII}=\mathrm{KKIII}+1$

$\mathrm{KKII}=\mathbf{K K}$

C

C GO THROUGH 210 LOOP FOR EACH PORTION OF RING BETWEEN FI(JI) AND FL(JI+1)

C

DO $210 \mathrm{JI}=1, \mathrm{IP}$

$\mathrm{JJI}=\mathrm{JI}+1$

$I F(J J I . G T . I P) ~ J J I=I$

ANG1 $=$ FLOAT $(J I-1) \star A N G$

ANG2 $=$ FLOAT $(J I) \star A N G$

$A M 111=F I(J J I) * S I N(A N G 2)-F I(J I) * S I N(A N G 1)$

IF (AM111.EQ.0.0) GO TO 205

$A M 11=F L(J J I) * \operatorname{COS}($ ANG2) $-E L(J I) * \operatorname{COS}(A N G 1)$

$\mathrm{AM} 1=\mathrm{AM} 11 / \mathrm{AM} 111$

$B I=F L(J I) *(\operatorname{COS}(A N G I)-A M I * S I N(A N G 1))$

C

$205 \mathrm{KI}=\mathrm{KKI}$

C GO THROUGH 140 FOR ALL ELEMENTS BETWEEN FL(JI) AND FI(JI+1)

C

DO $140 \mathrm{JJ}=\mathrm{KI}, \mathrm{JN}$

IF (ANG4.GT.ANG2 . OR. ANG4 . LE. ANG1) GO TO 210

$\mathrm{KKI}=\mathrm{KKI}+1$ 
IF(R33.GT.FL(JI) . AND.R33.GT.FI(JJI)) GO TO 140

C

C INTERCEPT BETWEEN FAULT PARAMETERS

C

IF (ANG4 .EQ.PI2.OR. ANG4 .EQ. (PI2/2.0)) GO TO 202

$\mathrm{AM} 2=\operatorname{COS}($ ANG4) $/ \mathrm{SIN}$ (ANG4)

$I E$ (AMI11.NE.0.0) XI = B1/ (AM2-AMI)

IF (AM111.NE.0.0) Y1 = AM1*X1 + B1

IF (AMI11.EQ.0.0) XI $=F I(J I) * S I N(A N G 1)$

$I F(A M 111 . E Q \cdot 0.0) \quad Y 1=A M 2 * X 1$

GO TO 203

$202 \times 1=0.0$

$Y 1=B 1$

203 INT $=\operatorname{SQRT}(X 1 \star \star 2+Y 1 * \star 2)$

IF (R33.GT.INT) GO TO 140

IF (ANG4 .GE. (PI2/4.) . AND.ANG4.IE. (.75*PI2)) GO TO 141

SURF $=A B S(A H /(\operatorname{SIN}(D P 1) * \operatorname{COS}($ ANG4) ))

IF (R33.GT.SURE) GO TO 140

C

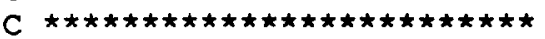

C EIEMENT WILL BE USED

C

$141 \mathrm{KJ}=\mathrm{KJ}+1$

$\mathrm{KK}=\mathrm{KK}+1$

C

C LOCATION OF ELEMENT

C

C AZMM IS HORIZONTAI PROJECTION OF AZIMUTH ALONG FAULT

C CAREFUL, ATAN GOES FROM 0 TO PI/2, -PI/2 TO PI/2, AND -PI/2 TO 0

C ATAN2 GO FROM 0 TO PI AND -PI TO 0

C ANG5 IS HORIZONTAL PROJECTION OF ANG4

C R4 IS HORIZONTAL LENGTH OF R3

C

ANG5 = ATAN2 (SIN (ANG4), $\operatorname{COS~(ANG4)~} * \operatorname{COS}(\mathrm{DP} 1)$ )

R4 $=$ R3*SQRT (SIN (ANG4) $* \star 2+\operatorname{COS}($ ANG 4$) \star \star 2 * \operatorname{COS}($ DP 1$) \star \star 2)$

AZMM $=$ STK1 - PI2/4. + ANG5

$\mathrm{RH}(\mathrm{KK})=\mathrm{AH}-\mathrm{R} 3 * \operatorname{COS}(\mathrm{ANG} 4) * \mathrm{SIN}(\mathrm{DP} 1$ )

RLAT $(\mathrm{KK})=\mathrm{ALAT}+\operatorname{COS}(\mathrm{AZMM}) * \mathrm{R} 4 * 360 . /(\mathrm{RHOM} * \mathrm{PI} 2)$

RLON $(K K)=A L O N-\operatorname{SIN}(A Z M M) * R 4 * 360 . /(R H O N \star P I 2 * \operatorname{COS}(A L A T * P I 2 / 360)$.

RIAT1 = RLAT (KK)

RION1 = RION (KK)

$\mathrm{RHI}=\mathrm{RH}(\mathrm{KK})$

ANG44 $=$ ANG $4 * 360 . /$ PI2

IF (ANG44.LT.0.0) ANG44 $=360.0+$ ANG 44

ANG55 $=$ ANG5 $* 360 . / P I 2$

IF (ANG55.IT.0.0) ANG55 $=360.0+$ ANG55

AZMM1 $=A Z M M * 360 . / P I 2$

IF (AZMM1.IT.0.0) AZMM1 = $360 .+$ AZMM1

C

C EIND SOURCE EVENT

C

$\mathrm{DH} 1=100.0$

DO $144 \mathrm{II}=1, \mathrm{NE}$

CALI DISAZM (AZM, AZINV, DIS, HDIS, BIAT (II) , BLON (II) , H, RLAT1, RLON1)

$H D I S=S Q R T(D I S * \star 2+(B H(I I)-R H I) * * 2)$

IF (HDIS.GT.DH1) GO TO 144

DHI $=$ HDIS

$E A A=E A(I I)$

$E B B=E B(I I)$

$\operatorname{INEI}(K K)=I I$

144 CONTINUE

$\mathrm{C}$

C COMPUTE P AND S DELAY TIME DUE TO IOCATION OF SOURCE EVENT 
BLAT1 = BLAT (INEL (KK))

BLONI $=$ BLON (INEL (KK) $)$

$B H 1=B H($ INEL $(K K))$

CALI DISAZM (AZM, AZINV, DIS, HDIS1, BLAT1, BLON1, BH1, SLAT, SION)

CAIL DISAZM (EZM, AZINV, DIS, HDIS2, RLAT1, RLON1, RH1, SLAT, SLON)

$\operatorname{RDIS}(\mathrm{KK})=\mathrm{DIS}$

$\mathrm{VP}=3.0+\mathrm{RHI} \star \mathrm{VPP}$

$\mathrm{VS}=\mathrm{VP} / 1.76$

$\mathrm{rgd}=\mathrm{vs}$

$\operatorname{DTIMP}(\mathrm{KK})=(\mathrm{HDIS} 2-\mathrm{HDIS1}) / \mathrm{VP}$

$\operatorname{ELTIM}(K K)=R 3 / V R$

$\operatorname{RAZM}(K K)=E Z M$

IF (SIM.NE.ANS3) GO TO 142

IF (IPA.EQ.1) RSTM $=(1.0 / \mathrm{VR}+1.0 / \mathrm{VH}) *(\mathrm{EL}(1)-\mathrm{R} 3)$

if (ipa.eq.3) rstm $=(1 . / \mathrm{vr}+1 . / \mathrm{vh}) *(\mathrm{aa} *(1 .-e c \star \star 2)-r 3 * e c *$

$\star \cos (p i 2 / 4$. -ang4-ann2) -r3)

IF (IPA.NE.1.and. ipa.ne.3) CALL RISTIM(VR, VH, FL, IP, R3, ANG4, RSTM)

$\operatorname{RISTM}(K K)=\operatorname{RSTM}$

$\operatorname{RDPP}(\mathrm{KK})=0.81 * \mathrm{STD} * \operatorname{SQRT}(\operatorname{RISTM}(\mathrm{KK}) * \star 2+$

$\star 2.0 *(\mathrm{R} 3 / \mathrm{VR}) \star \mathrm{RISTM}(\mathrm{KK}))$

amo $=$ amo + rdpp $(k k) * r g d * e l a$

IF (RDPP (KK) . GT.CPIT) ICPLT $=\mathrm{KK}$

IF (RDPP (KK) . GT . CPLT) CPLT $=$ RDPP (KK)

Di 42 IF (SIM.EQ.ANS4) RISTM(KK) = RTMHSK $\leftarrow$

DURTIM1 = RISTM(KK) + (R1+R2)/VR

IE (DURTIM1.GT.DURTIM) DURTIM = DURTIM1

sdurl $=r 33 / v r+\operatorname{ristm}(k k)+($ hdis2 - adis $) / v p$

if (sdurl.lt.sdur) go to 143

sdur = sour 1

143 dishsk = dishsk + ela*rgd*1.0e+21

C 146 IF (KKIII.EQ.3.AND.SIM.EQ.ANS4) WRITE (11,666) KK, ANG44,ANG55,

C 146 WRITE $(11,666)$ KR, ANG44, ANG55,

*AZMMI, EAA, EBB, DH1, DTIMP (KK) , RLAT1, RLON1, RH1

C IF (KKIII.EQ.3.AND.SIM.EQ.ANS3) WRITE (11,667) KK, ANG44, ANG55,

C *AZMM1, EAA, EBB, DH1, DTIMP (KK), RDPP (KK) , RISTM (KK)

140 CONTINUE

210 CONTINUE

666 FORMAT ('ELEM', I3,' ANG4=', F5.1,' ANG5=', F5.1,' AZMM=', F5.1, 3X, *'EVENT ', I3, 1X, I4,' DIS=', F5.2,' DTIMP=', F5.2,2X, $\star F 7.4,2 X, F 8.4,2 X, F 5.2)$

667 FORMAT (/'ELEM', I3,' ANG4=', F5.1,' ANG5=',F5.1,' AZMM=', E5.1, 3X, *'EVENT ',I3, 1X, I4,' DIS=',F5.2,' DTIMP=',F5.2,

* $\quad$ RDPP $=\prime, F 5.0, '$ RISTM $=\prime, F 5.2)$

IF (KJ.NE.O) GO TO 160

if (slm.ne.ans3) go to 145

amo $=a a^{\prime} * 1 \cdot 0 e+21$

std $=$ std*mo/amo

write $(11,1113)$ mo, amo

1113 format ('mo=', e10.3,' amo=', e10.3)

cplt $=$ cplt*mo/amo

do $1111 i=1, \mathrm{kk}$

$1111 \operatorname{rdpp}(i)=\operatorname{mo} \operatorname{rdpp}(i) / a m o$

WRITE $(11,112)$ STD

1112 format $(f 4.1,7 x, f 4.1,5 x, f 4.1,5 x, f 5.1,6 x, f 5.1)$

WRITE $(6,127)$ DURTIM,RISTM (1), RDPP (1), sdur

- WRITE $(11,127)$ DURTIM, RISTM(1), RDPP (1), sdur

WRITE $(6,123)$

WRITE $(6,126)$ Mo, amo

145 DISHSK $=$ MO/dishsk

IF (SLM.ne.ANS3) WRITE $(6,419)$ DISHSK, RTMHSK,DURTIM, SDUR >

IF (SLM.ne.ANS3) WRITE $(11,419)$ DISHSK, RTMHSK, DURTIM, SDUR

WRITE $(6,124) \quad \mathrm{KK}, \mathrm{RI}$

C

WRITE $(11,124) \mathrm{KK}, \mathrm{R} 1$ 


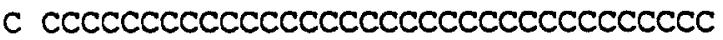

C

C BEGIN SYNTHESIS

C

C READ IN EACH FILE

C

PRINT *,,

WRITE $(6,302)$

PRINT *, $* \star \star$ READY TO BEGIN SYNTHESIS $\star \star \star * \prime$

IF (SIP. EQ. ANS6) GO TO 416

WRITE (11, 423)

423 FORMAT $(/ /$, 'SYNTHESIS IS BY CONVOLUTION OF SOURCE EVENTS WITH SLIP *FUNCTION,'/,'DECONVOLVED WITH STEP FUNCTION. EACH SOURCE EVENT IS * SPIIT BETWEEN P AND $s, \%, \cdot$ AND COMPUTATION IS DONE IN FREQUENCY DO *MAIN. FET FOR EACH SOURCE EVENT ONCE.')

IF (SIM.EQ.ANS3) WRITE $(6,418)$

IF (SIM.EQ.ANS3) WRITE $(11,418)$

$\mathrm{KK} 1=\operatorname{AMINO}(\mathrm{KK}, 160)$

IF (SIM.EQ.ANS3) WRITE $(11,135)$ KRI

IF (SIM.EQ.ANS3) $\operatorname{WRITE}(11,129) \quad(R D P P(I), I=1, K R 1)$

$\operatorname{IF}(S I M . E Q . A N S 3) \quad \operatorname{WRITE}(6,129) \quad(\operatorname{RDPP}(I), I=1, \mathrm{KK} 1)$

PRINT *,',

IF (FILOW.NE 0.0.AND.FIIHI.NE.100.0) WRITE $(6,427)$ FILOW, FILHI

IE (EIIOW.NE.0.0.AND.EILHI.NE.100.0) WRITE $(11,427)$ FIIOW, FIIHI

427 FORMAT ('OUTPUT BAND PASSED BETWEEN ', F4.1,' AND ',F5.1,' Hz')

PRINT *,"

$\mathrm{K}=8192$

$\mathbf{N}=13$

431 FORMAT ('SPECTRAL DIMENSION IS ', I4,' POINTS, IF TRACES ARE GREATER

* THAN ', I2,' SECONDS' $/$ ' YOU GET WRAP-AROUND IN MAIN SEISMOGRAM') GO TO 411

416 WRITE $(6,302)$

IE (SIM.NE.ANS4) WRITE $(6,422)$

IF (SIM.NE.ANS4) WRITE (11, 422)

422 FORMAT('SYNTHESIS IS BY INCREMENTING SLIP FUNCTION AND DELAYED STA *CKING')

IF (SIM.EQ.ANS3) WRITE $(11,417)$

IF (SIM.EQ.ANS3) WRITE $(6,417)$

417 FORMAT (/,'KOSTROV SIIP MODEL WITH HEALING'/,'DISPLACEMENT FIRST 1

*60 ELEMENTS (IEFT TO RIGHT)')

$\mathrm{KKI}=\mathrm{AMINO}(\mathrm{KK}, 160)$

$\operatorname{IF}(S I M . E Q . A N S 3)$ WRITE $(11,129)$ (RDPP (I), $I=1, K K 1)$

$\operatorname{IF}(S I M . E Q . A N S 3) \quad W R I T E(6,129) \quad(R D P P(I), I=1, K R 1)$

PRINT *, ,

IF (SIM.EQ.ANS3) RRINT *,'INTERVAL FOR INCREMENTING SLIP IS DT'

IF (SIM.EQ.ANS4) RRISS $=$ R1/VR

IF (SIM.EQ.ANS4) WRITE $(6,414)$ DISHSK, RRISS, sdur

IF (SLM.EQ.ANS4) WRITE (11,414) DISHSK, RRISS, sdur

414 FORMAT ///'TWO DIMENSIONAI HASKEI RUPTURE MODEL WITH STEP SLIP FUNC *TION'//'EACH ELEMENT HAS THE SAME DISPLACEMENT:',F5.1,' CM'/'TOTAI

* FAULTING DURATION',E5.2,' SECONDS' 1, 'STATION SOURCE PULSE DURATIO

*N: ',F5.2)

$\mathrm{K}=4000$

C

WRITE $(11,132) \mathrm{K}$

C SET SYNTHESIZED TRACE TO ZERO

C

411 DO $150 I=1,9000$

$\operatorname{TRZ}(I)=\operatorname{CMPIX}(0.0,0.0)$

$\operatorname{TRR}(I)=\operatorname{CMPLX}(0.0,0.0)$

$150 \operatorname{TRT}(I)=\operatorname{CMPIX}(0.0,0.0)$

C PARAMETERS FROM SOURCE FIIE:

C 
C TAMI: REAL TIME OF FIRST DATA POINT

C TAM2: SECONDS FROM ORIGAN OF EIRST DATA POINT

C LN: SECONDS OF RECORD IN ORIGINAI DATA FILE

C BAZM: BACK-AZIMUTH OE STATION FROM SOURCE EVENT

C SAZM: ORIENTATION OF TRACE TWO IN OUTPUT

C ROT: ROTATION OF STATION COORD W.R.T. NORTH (= BAZM)

C NP: LENGTH IN POINTS OF EACH TRACE

C DT: SAMPLING INTERVAL IN SECONDS

C SAZM: ORIENTATION OF TRACE TWO IN OUTPUT FILE

C EPI: EPICENTRAL DISTANCE

C ML: LOCAL RICHTER MAGNITUDE

C H: DEPTH OF EVENT

C $\operatorname{cccccccccccccc}$

C

C GO THROUGH 400 LOOR ONCE FOR EACH SOURCE EVENT, APPLY TO ALL

C ELEMENTS USING THE EVENT

C

C JJK TO COUNT ELEMENTS DURING COMPUTATION

$\mathrm{JJK}=0$

DO $400 \mathrm{JI}=1, \mathrm{NE}$

do $410 i=1, k$

$\operatorname{traz}(i)=0.0$

$\operatorname{trar}(i)=0.0$

410 trat $(i)=0.0$

$\mathrm{VQP}=3.0+\mathrm{BH}(\mathrm{JI}) * \mathrm{VPP}$

$\mathrm{VQS}=\mathrm{VQP} / 1.76$

IF (EB (JI) . LE. 999) III =0

IF (EB (JI) . GT . 999) III $=E B(J I) / 1000$

$I I 2=E B(J I)-I I 1 * 1000$

IF (II2.IE.99) II2 $=0$

IF(II2.GT.99) II2 $=I I 2 / 100$

$I I 3=E B(J I)-I I 1 * 1000-I I 2 * 100$

IF (II3.IE.9) II3 $=0$

IE (II3.GT.9) II3 $=I I 3 / 10$

II4 $=$ EB $(\mathrm{JI})-$ II1 * $1000-$ II2 * $100-I I 3 * 10$

$\mathrm{AEB}=\operatorname{CHAR}(\operatorname{II} 1+48) / / \operatorname{CHAR}(\operatorname{II} 2+48) / / \operatorname{CHAR}(\operatorname{II} 3+48) / / \mathrm{CHAR}(\operatorname{II} 4+48)$

NNSE $1=$ '

IF (SSC.EQ.ANS1.OR.SSC.EQ.ANS2) GO TO 412

OPEN (UNIT $=8$, EILE $=$ NNSF 1 )

READ (8, 401) ADAY, AHOUR, BHOUR, AMIN, BMIN, ASTA, BSTA, IN, TAM1

401 FORMAT (9X, A2, 1X, A1, A1, A1, A1, 12X, A1, A1, 18X, F5 .2, 14X, F5 .2)

IF (AHOUR.EQ.BLANK) AHOUR = ZERO

IF (AMIN.EQ.BLANK) AMIN = ZERO

IF (ASTA.EQ.BIANK) ASTA = ZERO

WRITE $(6,407)$

407 FORMAT $(/ / /)$

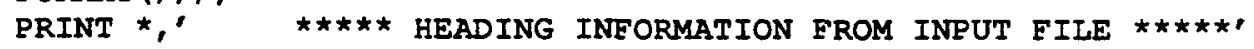

WRITE $(6,408)$ ASTA, BSTA, ADAY, AHOUR, BHOUR, AMIN, BMIN

$\operatorname{READ}(8,402)$ EPI, BAZM

402 FORMAT $(22 \mathrm{X}, \mathrm{F} 5.2,19 \mathrm{X}, \mathrm{F} 5.1 / / /)$

READ (8, 405) MI

405 FORMAT (28X, F3.1/)

WRITE $(6,403)$ EPI, BAZM

WRITE $(6,406) \mathrm{ML}$

406 FORMAT ('LOCAL RICHTER MAGNITUDE ',F3.1)

403 FORMAT('EPICENTRAL DISTANCE',F5.2,' EVENT-STAT AZIMU ',F5.1) $\operatorname{READ}(8,404)$ NP, DT, TAM2

404 FORMAT (15X, I 4, 20X, F6.4, 17X, F4.2//)

WRITE $(6,409)$ NP, DT, TAM2

409 FORMAT (/,'PTS $=$ ', I 4,' SAMPLING INTERVAL ',F6.4/,

*'FIRST DATA POINT SECONDS FROM ORIGIN ', F4.2//)

408 FORMAT $\left(/,{ }^{\prime}\right.$ STA ',A1,A1,' SAN FERNANDO 1971 0',A2, 1X,

$\star \mathrm{A} 1, \mathrm{~A} 1, \mathrm{~A} 1, \mathrm{~A} 1)$ 
$\operatorname{READ}(8,121) \quad(\operatorname{TRAZ}(I), I=1, \mathrm{NP}$ )

$\operatorname{READ}(8,123)$

$\operatorname{READ}(8,121)(\operatorname{TRAR}(I), I=1, \mathrm{NP})$

$\operatorname{READ}(8,123)$

$\operatorname{READ}(8,121)(\operatorname{TRAT}(I), I=1, \mathrm{NP})$ GO TO 413

412 NNSFI $1=$ ' $\mathrm{e}^{\prime} / / \mathrm{AEB} / / \mathrm{I}^{\prime} \mathrm{s} / / \mathrm{ASB} / /$ '.zZz'

CALL RSACI (NNSE1, TRAZ, NP, TAM2, DT, MAX, NERR)

NNSF1 $1=$ 'e'//AEB//'s'//ASB//' $\cdot \mathrm{rad}$

CALL RSAC1 (NNSF1, TRAR, NP, TAM2, DT, MAX, NERR)

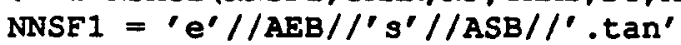

CALI RSAC1 (NNSF1, TRAT, NP, TAM2, DT, MAX, NERR)

$I T K=\operatorname{IFIX}($ FIOAT $(K) * D T-5.0)$

C WRITE $(11,431) \mathrm{K}, \mathrm{ITK}$

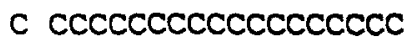

C COMPUTE RADIATION FACTOR FOR SOURCE EVENT

C

413 CALI DISAZM (BAZM, AZINV, BDIS, HDIS, BLAT (JI) , BLON (JI) , BH (JI) , SLAT

*, SLON)

CALL LINVEL (BLAT (JI) , BLON (JI) , SLAT, SLON, BH (JI) , BDIS , RP , ANGI,

*VSP, AINP, VZF)

$B Z M M=B A Z M-B S T R(J I)$

AANGI $=360 .{ }^{*}$ ANGI/PI2

AAINP $=360 . *$ AINP $/$ PI2

AINS $=\operatorname{ASIN}(\operatorname{SIN}(\operatorname{AINP}) / 1.76)$

VSS $=$ VSP $/ 1.76$

AAINS $=360 *$ AINS $/$ RI2

BSV1 $=($ BSV $(J I) / 360) * P I$.

$B Z M 1=(B Z M / 360) * P I$.

C

$\operatorname{BDP} 1=(\operatorname{BDP}(J I) / 360.) \star P I 2$

FFPP $=\operatorname{COS}(B S V 1) \star \operatorname{SIN}(B D P 1) *(\operatorname{SIN}(A N G I) \star \star 2) \star \operatorname{SIN}(2 . \star B Z M 1)-$

$\star \operatorname{COS}(B S V 1) \star \operatorname{COS}(B D P 1) \star \operatorname{SIN}(2 . \star A N G I) * \operatorname{COS}(B Z M 1)+\operatorname{SIN}(B S V 1) \star \operatorname{SIN}(2 . \star B D P 1)$

$\star \star((\operatorname{COS}($ ANGI $) * \star 2)-($ SIN (ANGI) $* \star 2) *($ SIN $(B Z M 1) * \star 2))+$

$\star \operatorname{SIN}(B S V 1) \star \operatorname{COS}(2 . \star \operatorname{BDP} 1) \star \operatorname{SIN}(2 . \star A N G I) * \operatorname{SIN}(B Z M 1)$

IF (FFPP .IT. 0. .AND.FFPP.GT. - .4) FFPP $=-.4$

IE (FFPP . GE . 0 . . AND. FEPP . IT . .4) FFPP $=.4$

IF (RDC.NE.ANS1.AND.RDC.NE.ANS2) FFPP $=1.0$

C

$\mathrm{EFSV}=\operatorname{SIN}(\mathrm{BSV} 1) \star \operatorname{COS}(2 . \star \mathrm{BDP} 1) \star \operatorname{COS}(2 . \star \mathrm{ANGI}) \star \operatorname{SIN}(\mathrm{BZMI})-\operatorname{COS}(\mathrm{BSV} I)$

$\star \star \operatorname{COS}(\mathrm{BDP} 1) \star \operatorname{COS}(2 . \star \mathrm{ANGI}) \star \operatorname{COS}(\mathrm{BZM} 1)+.5 * \operatorname{COS}(\mathrm{BSV} 1) \star \operatorname{SIN}(\mathrm{BDP} 1)$ *

$\star \operatorname{SIN}(2 . \star A N G I) \star \operatorname{SIN}(2 . \star B Z M 1)-.5 * \operatorname{SIN}(B S V I) \star \operatorname{SIN}(2 . * B D P 1)$ *

$\star \operatorname{SIN}(2, * A N G I *(1,+\operatorname{SIN}(B Z M 1) \star \star 2))$

IF (EFSV.IT. 0..AND.EFSV.GT.-.4) FESV $=-.4$

IF (EFSV.GE . 0 . . AND.FESV. IT . .4) FFSV $=.4$

C

IF (RDC.NE.ANS1.AND.RDC.NE.ANS2) FFSV $=1.0$

FFSH $=\operatorname{COS}(B S V 1) * \operatorname{COS}(B D P I) * \operatorname{COS}(A N G I) \star S I N(B Z M I)+$

*COS (BSVI) *SIN (BDPI) *SIN (ANGI) *COS (2 * $\operatorname{BZMI)+SIN~(BSVI)~*COS~(2~*~*BDP1)~}$

$\star \star \operatorname{COS}($ ANGI) $\star \operatorname{COS}(B Z M 1)-.5 \star \operatorname{SIN}(B S V 1) \star \operatorname{SIN}(2 . \star B D P 1) \star S I N(A N G I)$ *

$\star \operatorname{SIN}\left(2,{ }^{\star} \mathrm{BZM} 1\right)$

IF (EFSH . IT . 0 . . AND.FFSH.GT. - .4) FFSH $=-.4$

IE (EFSH.GE. 0 . AND.FESH.IT. .4) EFSH $=.4$

IF (RDC.NE.ANS1.AND.RDC.NE.ANS2) FFSH $=1.0$

C

WRITE (6, 305) EAA, AEB, BZMM, BDP (JI), BSV (JI), AANGI, AAINP, AAINS, *EFPP, FESV, FFSH

WRITE (11, 305) EAA, AEB, BZMM, BDP (JI) ,BSV (JI) , AANGI, AAINP, AAINS, *FEPP, FESV, FFSH

305 FORMAT $\left(/ /, * \star \star \star\right.$ SOURCE EVENT $\left(0^{\prime}, I 2, I X, A 4, \cdot\right) \star \star \star * \prime /$, *'FAULT-STAT AZI DIP SIIP VECTOR TAKEOFE ANGLE', * P S INCID ANGLES $/, 6 \mathrm{X}, \mathrm{F} 6.1,5 \mathrm{X}, \mathrm{F} 4.1,4 \mathrm{X}, \mathrm{F} 6.1,11 \mathrm{X}, \mathrm{F} 4.1,11 \mathrm{X}$, 
*F4.1,2X, F4.1/'RADIATION CORRECTION FACTORS

$\star /, 30 x$, F5.3, 3X, F5.3, 3X, F5.3)

c

ISS: POINTS INTO TRACE TO SPIIT P AND S ARRIVAIS

BHDIS $=$ SQRT (BDIS * *2+BH (JI) $* * 2)$

ISS $=\operatorname{IFIX((SPT~(JI)~}-$ TAM2)/DT)

WRITE (11, 424) SPT (JI), ISS

424 FORMAT ('SOURCE EVENT SPLIT FOR ANALYSIS ',F5.2,' SEC FROM ORIGIN', $\star$ ISS $=$ ', I4)

IF (SLP.EQ.ANS6) GO TO 435

$I T=\operatorname{IFIX}(.5 / D T)$

NT1 $=\mathrm{LT}+1$

NT2 = ISS - LT

NT3 $=$ ISS

NT4 = NP - IT

C BOTH TRACES MUST HAVE SAME K VALUE FOR CONVOLUTION WITH SLIP FUNCTION C MP AND MS ARE POINT SHIETS OF TRACES AFTER TAPERING

C

C AMPLITUDE FACTORS FOR COOL ARE APPLIED IN SUBROUTINE TAPER

SIGNI $=-1.0$

CAIL TAPER (NP , NT1, NT2, TRAZ, TZPZ, N, IT, MP, M1, K)

CALL COOL (N, TZPZ, SIGNI)

CALL TAPER (NP, NT1, NT2, TRAR, TZPR, N, LT, MP , M1, K)

CALL COOL (N, TZRR, SIGNI)

CALL TAPER (NP, NT1, NT2, TRAT, TZPT, N, LT, MP , M1, K)

$M P 1=M 1$

CALL COOL (N, TZPT, SIGNI)

CALL TAPER (NP, NT3, NT4, TRAZ, TZSZ, N, IT, MS, M1, K)

CAIL COOL (N, TZSZ, SIGNI)

CAIL TAPER (NP , NT3, NT4, TRAR, TZSR, N, IT, MS , M1, K)

CALI COOL (N, TZSR, SIGNI)

CALL TAPER (NP, NT3, NT4, TRAT, TZST, N, IT, MS , M1, K)

MS1 $=$ MI

CALL COOL (N, TZST, SIGNI)

$\mathrm{C}$

C NOW USE SOURCE EVENT FOR ALI ELEMENTS SPECIEYING THIS EVENT

435 DO $450 \mathrm{JK}=1, \mathrm{KK}$

IIN $=$ INEL $(\mathrm{JK})$

IF (IIN.NE.JI) GO TO 450

$\mathrm{VP}=3.0+\mathrm{RH}(\mathrm{JK}) * \mathrm{VPP}$

$\mathrm{VS}=\mathrm{VP} / 1.76$

RGD $=$ VS

IF (SIM.EQ.ANS4) RGD $=3.0$

IF (SIM.EQ.ANS3) SELM $=$ RDPP (JK)

IF (SIM.EQ.ANS4) SEIM $=$ DISHSK

$J J K=J J K+1$

AJK = FLOAT (JJK) $/ 10.0-$ FLOAT (IFIX (FLOAT $(J J K) / 10.0)$ )

IF (AJK.EQ.0.0) WRITE $(6,436)$ JJK, KK, JI, NE

C

436 FORMAT (I5,' ELEMENTS OF TOTAL ',I5,' SOURCE EVENT ', I2,' OE ', I2)

C IDEL: TOTAL DELAY IN POINTS FOR STACKING IN P PHASES

C IDELS: ADDITIONAL DELAY FOR S PHASES

C

$\operatorname{IDEL}=\operatorname{IFIX}(\operatorname{ELTIM}(J K) / D T+\operatorname{DTIMP}(J K) / D T+\operatorname{TAM} 2 / D T-S F O / D T)$

IF (IDEL.LT.0) WRITE $(6,425)$

IF (IDEL.LT.0) WRITE $(11,425)$

425 FORMAT ('WARNING: IDEL IT 0, SFO TOO IAARGE, DISTANCE CORRECTION'/, *'FACTOR WRONG, PROBABLEY VELOCITY USED FOR CORRECTION TOO SMALL'//

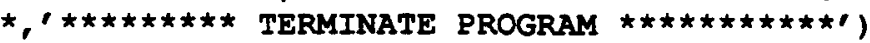

IF (IDEL.LT.O) GO TO 2000

C

IDELS $=\operatorname{IFIX}((D T I M P(J K) / D T) *(1.76-1.0))$ 
C SET ELEMENT TRACE TO ZERO

C

DO $445 I=1, K$

$\operatorname{ETRZ}(I)=\operatorname{CMPLX}(0.0,0.0)$

$\operatorname{ETRR}(I)=\operatorname{CMPLX}(0.0,0.0)$

C

$445 \operatorname{ETRT}(I)=\operatorname{CMPLX}(0.0,0.0)$

c

IF (SLP.EQ.ANS5) GO TO 455

C ROSTROV SLIP FUNCTION

C

KR: NUMBER OF INCREMENTS TO GET DESIRED RISE TIME. RISETIME INCREMENTED AT DIGITIZING RATE

SELM: TOTAL SLIP DESIRED FOR ELEMENT (NORMALIZED TO $1 . /\left(.3{ }^{\star} S T D * V R\right.$ )

SSEM: TOTAL SLIP OBTAINED FROM INCREMENTING (NORMAIIZED)

EAC1: SELM/SSEM TO GET DESIRED SLIP FOR ELEMENT

FAC2: SCALE SEISMOGRAM FOR INCREMENT OF SLIP FUNCTION

SS: PARTIAL SLIP AT ELEMENT

DELSS: INCREMENTAL STEP IN SLIP

C

C

IE (SLM.EQ.ANS3) SINC $=D T$

IF (SLM.EQ.ANS4) SINC $=0.0$

IF (SIM.EQ.ANS3) KR $=\operatorname{IFIX(RISTM(JK)/SINC~+.01)~}$

IE (SIM.EQ.ANS4) KR $=1$

IF (KR.EQ.0) KR $=1$

C

C

INCREMENTAL VALUES ARE SCALED TO PROVIDE EXACT DESIRED OFESET

SSEM $=0.81 * S T D * S Q R T(($ FLOAT $(K R) \star S I N C) \star \star 2+2.0 *$ FLOAT (KR) *

* SINC*ELTIM (JK))

IF (SLM.EQ.ANS3) EACI = SELM/SSEM

IF (SIM.EQ.ANS4) FAC1 $=1.0$

IE (SLM.EQ.ANS4) EAC2 $=$ RGD $* 1.0 E+11 \star E L A * 1.0 E+10 \star D I S H S K / B M(J I)$

SUM $=0.0$

$\operatorname{TIM}=0.0$

DO $460 I=1, K R$

IF (SIM.EQ.ANS4) GO TO 90

TIM $=$ TIM + SINC

SS $=$ EAC1 $* 0.81 * S T D * S Q R T(T-M * \star 2+2.0 * T I M \star \operatorname{ELTIM}(\mathrm{JK}))$

DELSS $=$ SS - SUM

SUM $=$ SUM + DELSS

C REMEMBER ELA $(\operatorname{KM} * \star 2)=\operatorname{ELA}(C M \star \star 2 * 1.0 E+10)$

FAC2 $=R G D * 1.0 E+11 * E L A * 1.0 E+10 * D E L S S / B M(J I)$

$\mathrm{NR}=\operatorname{IFIX}(\mathrm{TIM} / \mathrm{DT}+.001)$

90 IF (SIM.EQ.ANS4) NR $=1$

$K K I=0$

$\mathrm{NRR}=\mathrm{NR}+\mathrm{NP}-1$

DO 470 IK $=$ NR, NRR

$K K I=K K I+1$

$\operatorname{ETRZ}($ IK) $=\operatorname{ETRZ}(I K)+\operatorname{CMPIX}(\operatorname{TRAZ}(\mathrm{KKI}) *$ FAC2, 0.0$)$

$\operatorname{ETRR}(I K)=\operatorname{ETRR}(I K)+\operatorname{CMPLX}(T R A R(\mathrm{KKI}) \star F A C 2,0.0)$

470 ETRT $($ IK $)=\operatorname{ETRT}($ IK) $+\operatorname{CMPLX}(T R A T(K K I) \star E A C 2,0.0)$

C

460 CONTINUE

C APPLY RADIATION CORRECTION FOR ELEMENT

C

$455 D P 1=(D P / 360) * P I$.

$E Z M 1=(($ RAZM $($ JK $)-S T K) / 360) * P I$.

$S V 1=(S V / 360.) \star P I 2$

C

C NOW OTHER PARAMETERS

CALL LINVEL (RLAT (JK) , RLON (JK) , SLAT, SLON, RH (JK) , RDIS (JK) , RP, ANGI, 
$\star V S P$, AINP, VZF)

$\operatorname{EIPP}=\operatorname{COS}(\operatorname{SV} 1) \star \operatorname{SIN}(D P 1) *(\operatorname{SIN}(A N G I) \star \star 2) \star \operatorname{SIN}(2 . \star E Z M 1)-$

$\star \operatorname{COS}(\mathrm{SV} 1) * \operatorname{COS}(\mathrm{DP} 1) \star \operatorname{SIN}(2 . * A N G I) * \operatorname{COS}(\mathrm{EZM} 1)+\operatorname{SIN}(\operatorname{SV} 1) \star \operatorname{SIN}(2 . * \mathrm{DP} 1)$

$\star \star((\operatorname{COS}(A N G I) \star \star 2)-(\operatorname{SIN}(A N G I) * \star 2) *(\operatorname{SIN}(E Z M 1) \star \star 2))+$

*SIN (SV1) *COS (2. *DP1) *SIN (2. *ANGI) *SIN (EZM1)

IF (ELPP. IT . 0 . AND. ELPP . GT. . .4) ELPP $=-.4$

IF (ELPP . GE . 0 . . AND . ELPP . LT . .4) ELPP $=.4$

IF (RDC.NE.ANS1.AND.RDC.NE.ANS2) ELPP $=1.0$

C

$\operatorname{ELSV}=\operatorname{SIN}(\operatorname{SV} 1) \star \operatorname{COS}\left(2 .{ }^{\circ} \mathrm{DP} 1\right) \star \operatorname{COS}(2 . \star A N G I) * \operatorname{SIN}(E Z M 1)-\operatorname{COS}(\mathrm{SV} 1) \star$

$\star \cos (D P 1) \star \cos (2 . \star A N G I) * \cos (E Z M 1)+.5 * \cos ($ SVI) *SIN (DP1) *

$\star \operatorname{SIN}(2 . * A N G I) * \operatorname{SIN}(2 . \star \operatorname{EZMI})-.5 * \operatorname{SIN}(\operatorname{SV} 1) \star \operatorname{SIN}(2 . * D P 1)$ *

$\star \operatorname{SIN}(2 . * A N G I *(1 .+\operatorname{SIN}(\operatorname{EZM} 1) \star \star 2))$

IF (ELSV. LT. 0 . .AND.ELSV.GT. - .4) EISV $=-.4$

IF (ELSV. GE . 0 . AND . ELSV. LT . .4) ELSV $=.4$

C

IF (RDC.NE . ANS1.AND.RDC.NE. ANS2) ELSV $=1.0$

$\operatorname{ELSH}=\operatorname{COS}(\operatorname{SV} 1) * \operatorname{COS}(\mathrm{DP} 1) * \operatorname{COS}(\mathrm{ANGI}) * \operatorname{SIN}(E \mathrm{EM} 1)+$

*COS (SV1) *SIN (DP1) *SIN (ANGI) *COS (2. *EZMI) + SIN (SV1) *COS (2. *DP 1$)$ *

$\star \operatorname{COS}(A N G I) * \operatorname{COS}(E Z M 1)-.5 \star \operatorname{SIN}(S V 1) \star S I N(2 . * D P 1) * \operatorname{SIN}($ ANGI) *

$\star \operatorname{SIN}(2 . \star E Z M 1)$

IE (EISH. IT . 0 . AND.ELSH.GT. - .4) EISH $=-.4$

IF (ELSH.GE . 0. . AND.ELSH.IT. .4) ELSH $=.4$

IF (RDC.NE.ANS1.AND.RDC.NE.ANS2) ELSH $=1.0$

IF (JK.EQ.1) WRITE $(11,130)$

IE (JK.IT.10) WRITE $(11,131)$ ELPP, ELSV, ELSH

C

C

C ROTATE ETRZ (I),ETRR(I), ETRT(I) INTO STATION COORDINATES:

C

C PUT IN P AND S DELAY, SOURCE EVENT OT DELAY, RURTURE DELAY, AND

C ADD INTO SYNTHETIC SEISMOGRAM

C

C RAZM(JK) : AZIMUTH EROM ELEMENT TO STATION

C BAZM: AZIMUTH FROM SOURCE EVENT TO STATION

C

$A Z M 1=($ RAZM $(J K)-B A Z M) \star 0.017453$

SAZM1 $=($ SAZM $-\operatorname{RAZM}(J K)) \star 0.017453$

IF (SLP.EQ.ANS6) GO TO 420

C

C CONVOLVE SIIP FUNCTION APRLY PHASE DELAYS

C

C PHASE DELAY

APHP $=-1 . \star F L O A T(I D E L-M P) \star D T$

APHS $=-1 . \star$ FLOAT $($ IDEL + IDELS + (MS1-MP 1+1) - MS) *DT

C

C RADIATION CORRECTION

C

FAC4 $=E L A * R G D * 1 \cdot 0 E+11 * 1 \cdot 0 \mathrm{E}+10 / \mathrm{BM}(\mathrm{JI})$

FFAC1 $=$ CMPLX $(E A C 4 * E L P P / E F P P, 0.0)$

FFAC2 $=$ CMPLX $($ EAC $4 *$ ELSV/FFSV, 0.0$)$

FFAC3 $=$ CMPLX $($ EAC $4 * E L S H / F F S H, 0.0)$

E1 = REAL (EFAC1)

$F 2=\operatorname{REAL}($ FFAC2)

F3 = REAL (FEAC3)

C WRITE (11, 426) FAC4,F1, F2,F3

C

426 FORMAT('FAC4=',F7.3,'FFAC1=',F7.3,'FEAC2=', F7.3,' FFAC3=',F7.3)

IF (SIM.EQ.ANS4) RDPP (JK) = DISHSK

IE (RTMHSK.NE . 0.0) ASLP $=\operatorname{RDPP}(\mathrm{JK}) / \operatorname{RISTM}(\mathrm{JK})$

C

C RAMP SOURCE-TIME FUNCTION, DECONVOLVED WITH STEP FUNCTION OE GREEN'S 
$\mathrm{J} 1=\operatorname{IFIX}(\operatorname{FLOAT}(\mathrm{K}) / 2+1.001)$

C

C CORRECTION FACTORS FOR GEOMETRICAL SPREADING AND ATTENUATION

C

C $\operatorname{RHDIS}=\operatorname{SQRT}(\mathrm{RH}(\mathrm{JK}) \star \star 2+\operatorname{RDIS}(J K) \star \star 2)$

C REMEMBER WS IN LINEAR FREQUENCY

C WW IS ANGULAR FREQUENCY

$\mathrm{C}$

C SLIP FUNCTION IS ANALYTICALLY DECONVOLVED WITH A STEP FUNCTION

C

DO 430 IS $=1, \mathrm{~J} 1$

WW $=$ PI2*FLOAT $($ IS-1) $/($ FLOAT $(K) \star D T)$

RPFAC $=$ FFAC1 $*($ BHDIS $/$ RHDIS $) \star E X P(W W *(B H D I S-R H D I S) /(2 . * V Q P * Q))$

RRFAC $=$ FFAC2 $*($ BHDIS $/$ RHDIS $) * E X P($ WW $*(B H D I S-R H D I S) /(2 . * V Q S * Q))$

RTFAC $=$ FFAC $3 *($ BHDIS $/$ RHDIS $) \star E X P(W W *(B H D I S-R H D I S) /(2 . * V Q S * Q))$

ZPHP $=\operatorname{CMPLX}(\operatorname{COS}(W W \star A P H P), S I N(W W \star A P H P))$

ZPHS $=$ CMPLX $(\operatorname{COS}(W W \star A P H S), S I N(W W * A P H S))$

$\operatorname{IF}($ WW.NE.0.0) ESIP $=\operatorname{CMPIX}(0.0,(\operatorname{ASLP} / W W)) * \operatorname{CMPLX}(\operatorname{COS}(W W * \operatorname{RISTM}(J K))$,

$\star-\operatorname{SIN}(W W * \operatorname{RISTM}(J K)))-\operatorname{CMPIX}(0.0,(\operatorname{ASLP} / W W))$

IF (WW.EQ.0.0) ESLP $=\operatorname{CMPIX}(0.0,0.0)$

C NEXT FOR STEP FUNCTION

IF (RTMHSK. EQ.0.0) ESLP $=\operatorname{CMPLX}(\operatorname{RDPP}(\mathrm{JK}), 0.0)$

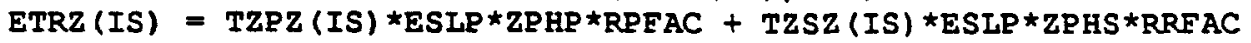

$\operatorname{ETRR}(I S)=T Z P R(I S) \star E S I P \star Z P H P \star R P F A C+T Z S R(I S) \star E S I P \star Z P H S \star_{R R P A C}$

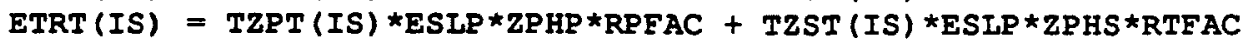

C NOW ROTATE TO RADIAL AND TANGENTIAL OF STATION

ETRA2 =ETRT (IS) *CMPLX (SIN (AZM1) , 0.0) +ETRR (IS) *CMPLX (COS (AZM1) , 0.0)

C ETRA3=ETRT (IS) *CMPLX (COS (AZM1) , 0.0) -ETRR (IS) *CMPIX (SIN (AZM1) , 0.0)

C NOW ROTATE TO SAZM FOR TRACE 2 AND SAZM + 90.0 FOR TRACE 3 TR2=ETRA $3 *$ CMPLX (SIN (SAZM1) , 0.0) +ETRA2 *CMPIX (COS (SAZM1), 0.0)

C TR3=ETRA $3 * C M P L X(C O S(S A Z M 1), 0.0)-E T R A 2 \star C M P I X(S I N(S A Z M 1), 0.0)$

C

$\operatorname{TRZ}(I S)=\operatorname{TRZ}(I S)+\operatorname{ETRZ}(I S)$

$T R R(I S)=T R R(I S)+T R 2$

$430 \mathrm{TRT}(\mathrm{IS})=\mathrm{TRT}(\mathrm{IS})+\mathrm{TR} 3$

C

C CONGIGATE OF TRZ, TRR, TRT DONE IN SUBROUTINE FILTER

C

GO TO 450

$C$

C APPLY RADIATION CORRECTION FOR ELEMENT

C

C

420 DO $480 I=1, \mathrm{~K}$

IF (I.LT.ISS) FFAC1 $=\operatorname{CMPLX}($ FFPP, 0.0$)$

IF(I.GE.ISS) FFAC1 $=$ CMPIX $(F F S H, 0.0)$

IF (I.IT.ISS) FFAC2 $=$ CMPLX (FFPP, 0.0)

IF (I.GE.ISS) FFAC2 $=$ CMPLX (FFSV, 0.0$)$

IF(I.LT.ISS) ELAC1 $=$ CMPIX (ELPP, 0.0)

IF (I.GE.ISS) ELACI $=$ CMPLX $(E L S H, 0.0)$

IF (I.IT.ISS) ELAC2 $=$ CMPLX (ELPP, 0.0)

IF (I.GE.ISS) ELAC2 = CMPLX (ELSV, 0.0)

C

ETRA1 $=\operatorname{ETRZ}(I) /$ FFAC2

C ROTATE TO STATION RADIAL AND TANGENTIAL ETRA2=ETRT (I) *CMPLX (SIN (AZMI), 0.0)/FFAC1

* + ETRR (I) *CMPLX $(\operatorname{COS}(A Z M 1), 0.0) /$ FFAC2 
C NOW ROTATE TO SAZM AND SAZM + 90.0

TR1 = ETRA1 *ELAC2

TR2 $=$ ETRA $3 \star_{\text {ELAC1 }} *_{C M P L X}($ SIN $($ SAZM1), 0.0$)$

* + ETRA2 *ELAC2*CMPLX (COS (SAZM1) , 0.0)

TR3 = ETRA3 $\star_{\text {ELAC1 }} \star_{C M P L X}(\operatorname{COS}($ SAZM1), 0.0$)$

C

* - ETRA2*ELAC2 *CMPLX (SIN (SAZM1), 0.0)

C

IDEI $=$ IDEI + 1

IF (I.EQ.ISS) IDEL = IDEL + IDELS

TRZ (IDEL) $=$ TRZ (IDEL) + TRI

$T R R(I D E L)=T R R(I D E L)+T R 2$

480 TRT (IDEL) $=$ TRT (IDEL) + TR3

450 CONTINUE

400 CONTINUE

IF (SLP.EQ.ANS5) GO TO 485

GO TO 490

485 CALL FILTER (TRZ, K, DT, FILOW, FILHI)

CALI FILTER (TRR, K, DT, FILOW, FILHI)

CAII FILTER (TRT, K, DT, FILOW, FILHI)

SIGNI $=1.0$

CALI COOL (N, TRZ, SIGNI)

CAIL COOI (N, TRR, SIGNI)

CALI COOL (N, TRT, SIGNI)

C IF DWN IS YES, THEN DOWN FOR COMPRESSION AND TRAZ IS REVERSED

490 DO $486 \mathrm{I}=1, \mathrm{~K}$

IF (DWN.EQ. ANS1 .OR.DWN.EQ.ANS2) TRZ (I) $=-T R Z(I)$

$\operatorname{TRAZ}(I)=\operatorname{REAL}(\operatorname{TRZ}(I))$

$\operatorname{TRAR}(I)=\operatorname{REAL}(\operatorname{TRR}(I))$

$486 \operatorname{TRAT}(I)=\operatorname{REAL}(\operatorname{TRT}(\mathrm{I}))$

NNSF2 $=$ ' $m$ ' //ALPHSYN//'s' //ASB

NNSF $3=$ NNSF $2 / /^{\prime}, \mathrm{zZZ}^{\prime}$

IF (DWN.EQ . ANS1 . OR.DWN.EQ. ANS2) NNSF3 $=\mathrm{NNSE} 2 / / /^{\prime}$.dWn'

CALI WSACI (NNSF 3, TRAZ, K, SFO, DT, NERR)

$\operatorname{ICMPI}=\operatorname{IFIX}($ SAZM/100.)

$\operatorname{ICMP2}=\operatorname{IFIX}(($ SAZM-FLOAT $(\operatorname{ICMP} 1 * 100)) / 10$.

ICMP $3=\operatorname{IFIX}($ SAZM-FLOAT (ICMP 1*100) -FLOAT (ICMP2*10))

NNSF $3=\operatorname{NNSE} 2 / /, \cdot / /$ CHAR $($ ICMP $1+48) / /$ CHAR $($ ICMP $2+48) / /$

*CHAR (ICMP 3+48)

CALI WSAC1 (NNSE 3, TRAR, K, SFO, DT, NERR)

ICMP $1=\operatorname{IFIX}($ SAZMM/100.)

ICMP2 $=\operatorname{IFIX}(($ SAZMM-FLOAT $(\operatorname{ICMP} 1 * 100)) / 10$.)

ICMP $3=\operatorname{IFIX~(SAZMM-FLOAT~}($ ICMP 1*100)-FLOAT (ICMP $2 * 10))$

NNSF $3=\operatorname{NNSF} 2 / / \prime \prime / /$ CHAR $($ ICMP $1+48) / /$ CHAR $($ ICMP2 +48$) / /$

*CHAR (ICMP 3+48)

CALI WSAC1 (NNSF 3, TRAT, K, SFO, DT, NERR)

CALI PLOTA (STK, DP, RLAT, RLON, RH, ALAT, ALON, AH, FL, IP, CPLT, ICPLT,

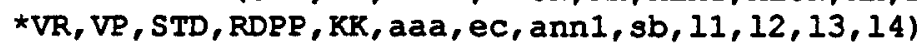

IF (DP .LT . 45.) CALI PLOTB (STK, DP, RLAT, RLON, RH, ALAT, ALON, AH, FI, IP, *CPLT, ICPLT, VR, VP, STD, RDPP, KK, inV)

CALI PLOTC (STK, DP, FI, IP, BLAT, BLON, ALAT, ALON, NE, SLAT, SLON, SB, AH)

2000 STOP

CALI PLOT $(9.0,9.0,999)$

END 


\section{Bibliography}

Aki, Keiiti and Paul G. Richards (1980) Quantitative Seismology, Theory an Methods, Volumes I and II, W. H. Freeman and Company, San Francisco.

Allen, Clarence R., Thomas C. Hanks, and James H. Whitcomb (1973) San Fernando earthquake: seismological studies and their tectonic implications; in San Fernando, California, Earthquake of February 9, 1971, Vol III Geological and Geophysical Studies, U.S. Department of Commerce, pp 13-22.

Brune, J.N. (1970) Tectonic stress and the spectra of seismic shear waves from earthquakes, J. Geophys. Res. 75, pp 4997-5010.

Brune, J.N. (1971) Correction, J. Geophys. Res. 76, no 20.

Buridge, R. and J.R. Willis (1969) The self-similar problem of the expanding crack in an anisotropic solid, Proc Cambridge Phil Soc, Vol 66, pp 443-468.

CIT (1972) Strong motion earthquake accelerograms, Vol II corrected, corrected accelerograms; Engineering Research Laboratory, California Institute of Technology (1969-1975).

Dahlen, F. A. (1974) On the ratio of P-wave to S-wave corner frequencies for shallow earthquake sources, Bull Seis Soc of Am, Vol 64, pp 1159-1180.

Hadley, D.M. and D.V. Helmberger (1980) Simulation of strong ground motion. Bull Seis Soc of $A m$, Vol 70, no. 2.

Hanks, T.C. (1974) The faulting mechanism of the San Fernando earthquake, $J$ Geophys Res, Vol 79, pp 1215-1229.

Hartzel, S. H. (1978) Earthquake Aftershocks as Green's Functions, Geophysical Research Letters, Vol 5, No 1

Hartzel, S. H. (1982) Simulation of ground accelerations for May 1980 Mammoth L:akes, California, earthquakes, Bull Seis Soc of Am, Vol 72, pp 2381-2387.

Heaton, T. H. (1978) Generalized Ray Models of Strong Ground Motion, Ph.D. thesis, Calif. Instit. of Tech., Pasadena, California.

Heaton, T. H. (1982) The 1971 San Fernando Earthquake: A Double Event?, Bull Seis Soc $m$, Vol 72, pp 2037-2062.

Hutchings, L. J. (1987), Modeling near-source earthquake ground motion with empirical Green's functions. Ph.D. Dissertation. Department of Geological Sciences, State University of New York, Binghamton, New York, 180 pp.

Irikura, K., and I. Muramatu (1983), Synthesis of strong ground motion from large earthquakes using observed seismograms of small events, Proc. $3^{\text {ed }}$ International Earthquake Microzonation Conf., Seattle, 447-458.

Johnson, L. R. (1974) Green's Function for Lamb's Problem, Geophy. Jour Roy Astro Soc, Vol 37, pp 99-131.

Kostrov, B.V. (1964) Selfsimilar problems of propagation of shear cracks, J Appl Math Mech (PMM), Vol 28, pp 1077-1087.

Langston, Charles A. (1978) The February 9. 1971 San Fernando earthquake: a study of source finiteness in teleseismic body waves, Bull Seis Soc of Am, Vol 68, pp 1-29. 
Munguia, L. and Brune, N. B. (1984) Simulation of strong ground motion for earthquakes in the Mexicali-Imperial Valley region. Geophys. J. R. astr. Soc. 79, 747-771.

Savage, J. C. (1972) Relation of corner frequencies to fault dimensions, J Geophys Res, Vol 77, pp 3788-3795.

Wu, F. T. (1978) Prediction of Strong Ground Motion using Small Earthquakes, Proceedings of the 2nd International Microzonation Conference, Vol 2, 701-704. 